\title{
Arrhythmia management by the implantable dual chamber defibrillator : possibilities and limitations
}

Citation for published version (APA):

Dijkman, B. (2001). Arrhythmia management by the implantable dual chamber defibrillator : possibilities and limitations. [Doctoral Thesis, Maastricht University]. Datawyse / Universitaire Pers Maastricht. https://doi.org/10.26481/dis.20010921bd

Document status and date:

Published: 01/01/2001

DOI:

10.26481/dis.20010921bd

Document Version:

Publisher's PDF, also known as Version of record

\section{Please check the document version of this publication:}

- A submitted manuscript is the version of the article upon submission and before peer-review. There can be important differences between the submitted version and the official published version of record.

People interested in the research are advised to contact the author for the final version of the publication, or visit the DOI to the publisher's website.

- The final author version and the galley proof are versions of the publication after peer review.

- The final published version features the final layout of the paper including the volume, issue and page numbers.

Link to publication

\footnotetext{
General rights rights.

- You may freely distribute the URL identifying the publication in the public portal. please follow below link for the End User Agreement:

www.umlib.nl/taverne-license

Take down policy

If you believe that this document breaches copyright please contact us at:

repository@maastrichtuniversity.nl

providing details and we will investigate your claim.
}

Copyright and moral rights for the publications made accessible in the public portal are retained by the authors and/or other copyright owners and it is a condition of accessing publications that users recognise and abide by the legal requirements associated with these

- Users may download and print one copy of any publication from the public portal for the purpose of private study or research.

- You may not further distribute the material or use it for any profit-making activity or commercial gain

If the publication is distributed under the terms of Article $25 \mathrm{fa}$ of the Dutch Copyright Act, indicated by the "Taverne" license above, 
Arrhythmia Management by the

Implantable Dual Chamber Defibrillator

- Possibilities and Limitations 
Production by Datawyse / Universitaire Pers Maastricht ISBN 9052783055

(C) Barbara Dijkman, Maastricht 2001 


\section{Arrhythmia Management by the Implantable Dual Chamber Defibrillator - Possibilities and Limitations}

\section{Proefschrift}

ter verkrijging van de graad van doctor

aan de Universiteit Maastricht,

op gezag van de Rector Magnificus,

Prof. Dr. A.C. Nieuwenhuijzen Kruseman, volgens het besluit van de College van Decanen, in het openbaar te verdedigen op vrijdag 21 september 2001 om 16.00 uur

door

\section{Barbara Dijkman}

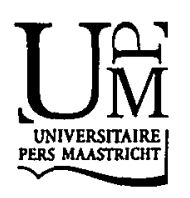




\section{Promotor}

\section{Prof.dr. H.J.J. Wellens}

Beoordelingscommissie

Prof.dr. H.J.G.M. Crijns (voorzitter)

Dr. A.P.M. Gorgels

Prof.dr. R.N.W. Hauer (Universiteit Utrecht)

Prof.dr. L. Jordaens (Erasmus Universiteit Rotterdam)

Prof.dr. B. Mochtar

Financial support by the Netherlands Heart Foundation for the publication of this thesis is gratefully acknowledged.

Additional support was granted by Guidant BV, Medtronic BV, Vitatron BV and the Rescar Foundation. 


\section{Contents}

INTRODUCTION

CHAPTER 1

ICD Technology - Development and Current Status

CHAPTER 2

ICD Therapy in Maastricht Academic Hospital

\section{CHAPTER 3}

Dual Chamber Arrhythmia Detection in the Implantable Cardioverter Defibrillator Journal of Cardiovascular Electrophysiology, 2000; 11:1105-1115.

\section{CHAPTER 4}

Importance of the Atrial Channel for Ventricular Arrhythmia

Therapy in the Dual Chamber Implantable Cardioverter Defibrillator

Journal of Cardiovascular Electrophysiology, 2000; 11:1309-1319.

\section{CHAPTER 5}

Diagnosis and Therapy of Atrial Tachyarrhythmias in the

Dual Chamber Implantable Cardioverter Defibrillator

Journal of Cardiovascular Electrophysiology, 2000; 11: 1196-1205.

\section{CHAPTER 6}

Merits and Limitations of the Mode Switching Rate Stabilization Pacing Algorithms in the Implantable Cardioverter Defibrillator Journal of Interventional Cardiac Electrophysiology, in press.

\section{CHAPTER 7}

Atrial Therapies Reduce Atrial Arrhythmia Burden in Defibrillator Patients Circulation, in press.

\section{CHAPTER 8}

Interactions Between Pacing and Arrhythmia Detection Algorithms in the Dual Chamber Implantable Cardioverter Defibrillator 
CHAPTER 9

General Discussion

SUMMARY

SAMENVATTING

DANKWOORD

CURRICULUM VITAE

PUBLICATIONS 


\section{Introduction}

The place of the implantable cardioverter defibrillator (ICD) has changed during the past twenty years. Initially, the purpose of the first defibrillators was to recognize and timely interrupt lethal ventricular arrhythmias. Thereafter new functions were continuously added to extent and improve treatment of different cardiac arrhythmias. This resulted in the growing use of the device to manage complex supraventricular and ventricular rhythm disturbances.

Dual chamber ICD technology played an important role in this development. In this thesis the experience is described of arrhythmia treatment using ICD therapy in the Academic Hospital Maastricht, with the emphasis on the study of the dual chamber technology. In particular, how the new algorithms for arrhythmia detection, therapy and prevention, which became available in the dual chamber ICD, advanced management of cardiac arrhythmias using implantable devices. 
[8] 
CHAPTER 1

ICD Technology - Development and Current Status 


\section{HISTORICAL MILESTONES}

The first human heart defibrillation was performed in 1788 using Kite's electrostatic generator saving life of a 3 years old child (1). It took another century before sudden death was linked to ventricular fibrillation (VF) in MacWilliams' publication in the British Heart Journal in 1889. Beck et al. demonstrated in 1947 that $110 \mathrm{~V}$ alternating current (AC) applied directly to the human heart could successfully convert VF during thoracic surgery. Transthoracic defibrillation was achieved by Zoll et al. in 1954 when they applied AC current via copper electrodes to the chest of a patient to terminate cardiac arrest. In the same year Hopps accomplished successfully transvenous defibrillation in animal studies. In 1962 Lown et al. used a capacitor to store direct current and demonstrated that its discharge was as effective as AC for terminating tachyarrhythmias and significantly less arrhythmogenic. The concept of cardiac defibrillation received an additional dimension after Pantridge et al. demonstrated in 1967 the feasibility of out-of-hospital resuscitation. With the advent of coronary units (CCU) in the early 1960s, external defibrillation and cardioversion became more and more commonplace in hospital. During the mid-1970s, Liberthson (2) and Baum (3) demonstrated that VF was the predominant cause of out-of-hospital sudden cardiac death (SCD), and that prompt defibrillation was essential to achieve higher salvage rates. Furthermore, it became recognized that the high recurrence rate of cardiac arrest was due to ventricular arrhythmias $(2,3)$. These findings initiated the extensive use of ambulance and bystander involvement in cardiopulmonary resuscitation, to reach more patients within the critical few minutes after their cardiac arrest.

Dr. Mirowski's concept of an implantable defibrillator began in 1967 with the sudden death of his mentor and close personal friend, Professor Harry Heller, who suffered from recurrent ventricular tachycardia (VT). At that time with an external defibrillator weighing $15-20 \mathrm{~kg}$, it was almost impossible to think of reducing its size to make it an implantable device. However, Dr. Mirowski was challenged by the problem, "because of the death of a man I admired very much, but also because people told me it couldn't be done" (4). Dr. Mirowski became in 1969 the head of the Coronary Care Unit in Sinai Hospital in Baltimore. Together with Dr. Mower, his friend and major contributor to the project, they built the first experimental automatic defibrillator model in the basement of the hospital, without any institutional or outside financial support. Mirowski's group developed and tested several electrode systems, some of them were partially and some completely intravascular and demonstrated the feasibility of terminating VF with energies below $20 \mathrm{~J}$. Interestingly, for sensing of the arrhythmia a right ventricular pressure transducer was incorporated. With the technology available in the early 1970 s, the first prototype of a device weighted nearly 300 $\mathrm{g}$ and had a volume of $150 \mathrm{cc}$. At that time the current drain was too high for a long battery life. 
From the very beginning the idea of an automatic implantable defibrillator was poorly received by both the cardiological and engineering communities. The need for such a device was not recognized and the validity of the assumption underlying this concept was vigorously questioned. While Mirowski and his co-workers were one-by-one overcoming the technological obstacles, they encountered two major setbacks in 1972. The first was a highly critical editorial in Circulation by Dr. Bernard Lown, in which he stated, "it is unlikely that VF will be induced deliberately to ascertain performance of the implanted devices"(5). The second was the decision, by the pacemaker company who had been working on the project, to drop it, essentially deeming the "market" to small and the technical problems to great. So Mirowski and co-workers found themselves not only without outside funding, but also opposed conceptually in their own field. Fortuitously, that same year, Dr. Mirowski met Dr. Stephen Heilman, a physician and engineer, whose angiography company (Medrad, later Intec Systems Pittsburgh) took an interest in providing technical support. That played a decisive role in transforming the early experiments into a reliable clinical device. By 1976 Mirowski's group had the model ready for long-term implantation and testing in dogs. Rather then another paper, they made a movie showing the experimental fibrillation, arrhythmia detection and defibrillation sequences in nonanethesized dogs. The arrhythmia and its conversion could be clearly correlated with the dog's collapse and recovery. The dog's ECG was added along the bottom of the film synchronized to the different stages. Mirowski felt, that this movie seen by many medical meetings in the late 1970s played a major role in overcoming the reigning skepticism. In the next four years environmental stress testing as well as long-term implantation in animals were carried out to qualify the device for human use. During that time, the experimental observations performed in the operating room validated the effectiveness of intrathoracic defibrillation using shocks with ten-fold less energy than the ones required for transthoracic defibrillation (6). Studies were also performed to determine optimal electrode positioning and energy levels for internal defibrillation in humans. There were some negative aspects of the initially used transvenous approach. These included the high-energy requirements, the theoretical possibility of myocardial damage from high-current density through a relatively small electrode surface area, venous thrombosis and the possibility of electrode migration $(7,8)$. The electrical sensing mechanism replaced the haemodynamical, allowing direct and rapid detection of arrhythmias. The experiments indicated that by far the lowest, and most stable energy requirements for successful defibrillation were achieved with insulated patch electrodes placed directly on the heart. Following extensive laboratory testing of the defibrillator function and longevity, the US Federal Food and Drug Administration (FDA) gave permission to begin human trials. With the first implant on February 4,1980 at the Johns Hopkins Medical Center in Baltimore the greatest task had been accomplished. The first clinical trial on feasibility, safety and clinical value of an 
implantable cardioverter defibrillator (ICD) lasted 5 years during which 800 defibrillators were implanted, resulted in FDA approval for market release. Even in those early days the initial findings were far from what the literature would have predicted about the prognosis of patients with repeated cardiac arrest $(9,10)$.

\section{ICD HARDWARE- IMPACT ON THE SURGICAL PROCEDURE}

From the time of the initial concept in 1967 through the next several years, Dr. Mirowski's presentations often alluded to the need for an "implantable CCU". That idea, which seemed preposterous three decades ago, becomes possible with modern devices. The ICD lead system has to carry out the function of rhythm sensing, antibradycardia and antitachycardia pacing and the delivery of high voltage therapy for defibrillation and cardioversion.

The first fully epicardial lead system consisted of two large epicardial patches for defibrillation and two epicardial screw-in leads for ventricular rate sensing (fig 1). Dr. Mirowski's first concept of a non-thoracotomy lead system was achieved in 1987. A triple electrode system was used for defibrillation, consisting of a right ventricular coil (RV coil), a superior vena cava/right atrium coil (SVC) and a subcutaneous patch (SQ) in the left lateral region (11). The device had a weight of $250 \mathrm{mg}$ and a volume of $140 \mathrm{cc}$. The development of the lead system, not requiring an open chest procedure has overcome one of the major obstacles to widespread acceptance of the therapy by greatly reducing concomitant operation risks, recovery and hospitalization time. This made ICD therapy available also for patients, who were previously considered to be at too high an operative risk. Unfortunately there were still some $15-30 \%$ of patients, in whom a nonthoracotomy ICD system was not feasible because of a too high defibrillation threshold (12-15). Modifications of the waveform of the defibrillation pulse and the introduction of the biphasic shock allowed reducing the energy requirements by about $30 \%$ (16-18). The biphasic shock applied in Ventitrex devices was an important development, because the nonthoracotomy systems became feasible in practically all patients.

The nonthoracotomy lead systems themselves have undergone a major evolution. Initially they required multiple access sites and tunneling, for the generator pocket was in the abdominal region (fig 2). The first generation of a nonthoracotomy lead design in 1986 allowed both the RV coil and the SVC coil to be integrated within one lead body, but had a high failure rate because of fracture (19). As a result of the high fracture rate the widespread clinical use of nonthoracotomy lead systems was delayed for several years. In the early 1990s the nonthoracotomy leads were redesigned. Pectoral implants became feasible in 1993, after the device volume could be reduced to $80 \mathrm{cc}$. The first pectoral transvenous ICD systems required at least two defibrillation elec- 
trodes, mostly the RV and the SVC coils (20). By including the pulse generator housing as part of the defibrillation circuit in 1993 the electrode surface area was increased and the energy requirements for arrhythmia termination decreased. An active can ("hot can") together with a single coil RV lead became the most simple endocardial ICD system (fig 3), allowing further reduction of the surgery time (21). The reduction of a device volume to $40 \mathrm{cc}$ (1999) in single chamber devices encouraged subcutaneous implantation in the electrophysiology (or the catheterization) laboratory, using conscious sedation instead of general anesthesia. While the volume of the single chamber ICDs decreased, the appearance of the dual chamber ICD technology in 1995 resulted again in increased size of those devices and more extensive lead systems. In 1996 the ICD having atrial arrhythmia management possibilities was developed, and the lead system was often extended with an extra electrode placed in the coronary sinus or a subcutaneous array in the left posterior region in order to lower the atrial cardioversion threshold (fig 4). In ICDs with biventricular pacing capabilities, which were in introduced in 1999, the lead system was extended with an additional pacing electrode placed in the coronary sinus (fig 5). Uncommonly, also in modern endocardial lead systems auxiliary subcutaneous array leads are still necessary $(22,23)$ to lower the VF defibrillation threshold by reaching the more posterior region of the heart or creating a more optimal defibrillation pathway.

\section{TACHYARRHYTHMIA SENSING}

Correct rhythm sensing in the ICD is of paramount importance. The primary challenge is the ability to adjust the sensing level in such a way, that it allows reliable sensing of low amplitude electrograms during VF, while oversensing of intracardiac and extracardiac signals during sinus rhythm is avoided.

In the first generation of ICDs the sensing mechanism was based on the analysis of the probability density function specific for VF. The logic circuit measured the time spent by the input electrogram between two amplitude limits located near the zero potential. VF was characterized by an absence of isoelectric potential segments. In the second AID-B model physicians were offered a range of cut-off rates that were installed at the factory, as well as the option between "rate only" and "rate plus probability density function" for detection of ventricular arrhythmias. A separate rate sensing lead was added, although in early models it was connected with one of the patches.

In modern ICDs sophistication of sensing was achieved in two ways (fig 6). The Automatic Gain Control, applied in Guidant ICDs, attempts to maintain a constant signal amplitude for any input signal and uses a fixed threshold to sense the $\mathrm{R}$ waves. The amplifier increases the gain when the amplitudes of several $R$ waves decrease from large values during sinus rhythm to smaller values during VF. It means, that in auto- 


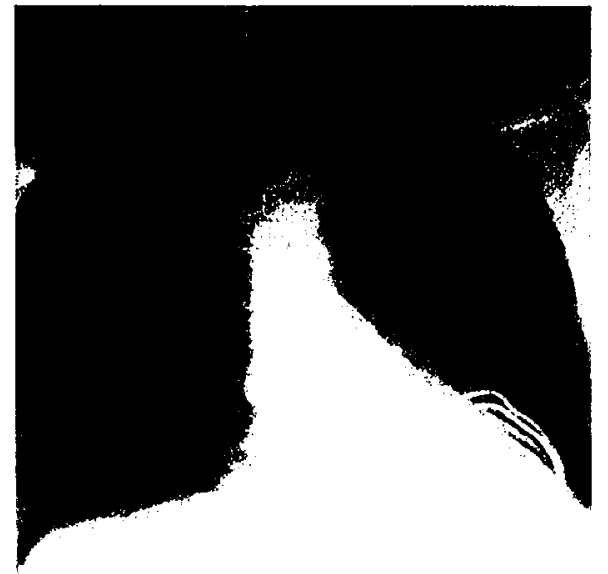

Figure 1. Chest $X$-ray showing the epicardial lead system. Two patches, placed anteriorly and infero-laterally are used for high voltage therapy and two screw-in epicardial electrodes apically on the left ventricle are used for sensing and pacing. The ICD is in the abdominal pocket (not visible in this early postoperative $\mathrm{X}$-ray).

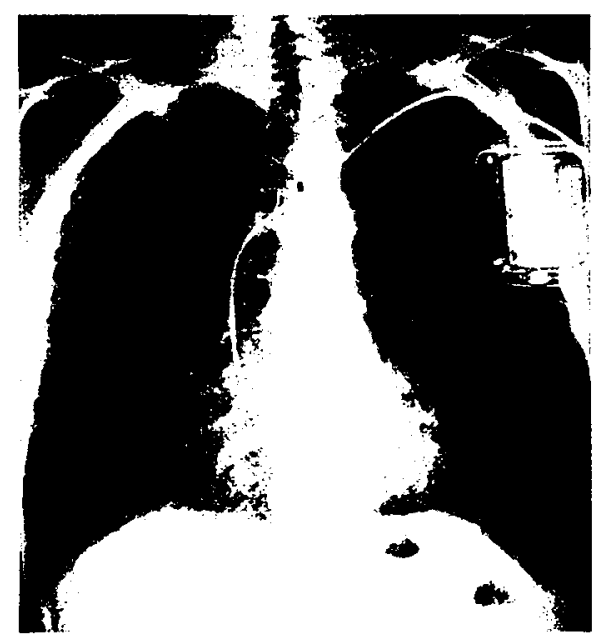

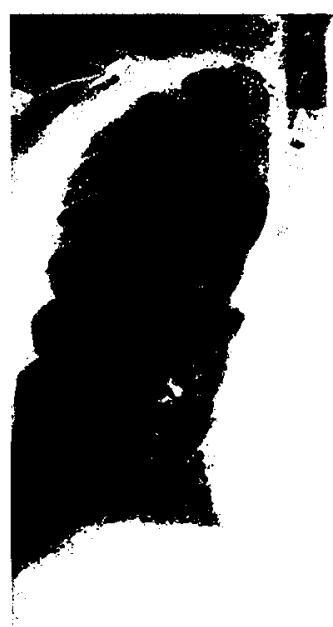

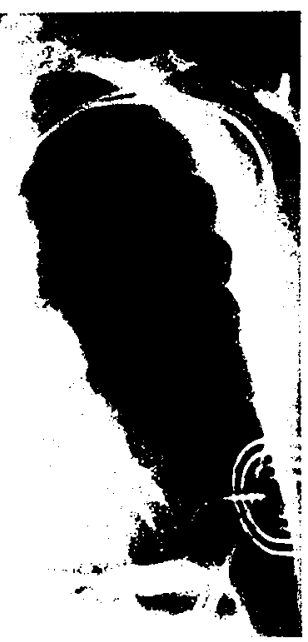

Figure 2. Chest $X$-ray showing the non-thoracotomy lead system. Three leads are used for high voltage therapy: the right ventricle (RV) coil, the superior vena cava (SVC) coil and the subcutaneous (SQ) patch in the left lateral region. The tip-ring dipole of the distal RV lead became the bipolar sensing and pacing lead. The RV electrode is introduced via the cephalic vein, the SVC via the subclavian vein. The three leads are tunneled to the left abdominal region, where the submuscular ICD pocket is present.

Figure 3. Chest $X$-ray showing the pectoral single lead system. This is the most simple transvenous system in which the high voltage electrodes are the active can and the single RV coil, the sensing and pacing dipole are the tip-ring of the same RV lead. 

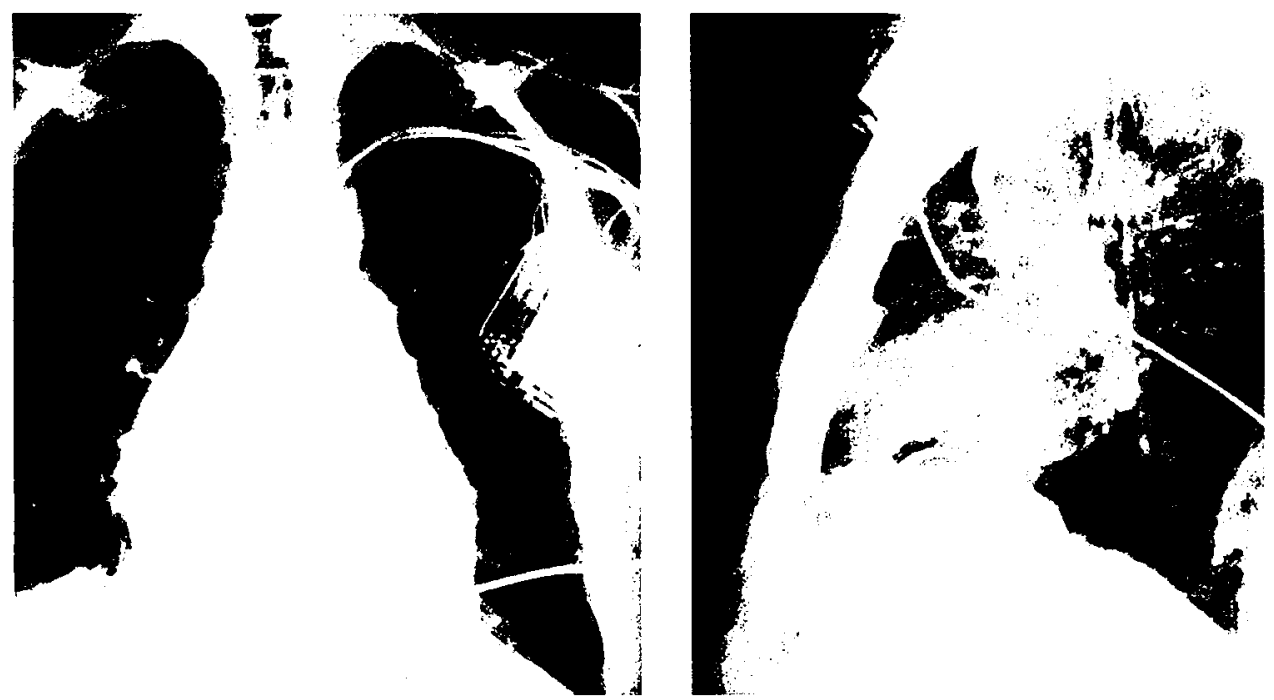

Figure 4. Posteroanterior and lateral chest $X$-ray showing a dual chamber ICD for atrial and ventricular arrhythmia management. The high voltage part of the system consists of the RV coil, the SVC coil, the SQ array left posterior and the active can. The ventricular pace/sense dipole is formed by the tip-ring of the RV lead; the atrial pace/sense dipole is formed by the tip-ring of the right atrial lead. The high voltage therapy configuration and pathway are programmable separately for each of the available therapy tiers. In later systems both the RV coil and the SVC coil are integrated within one lead.
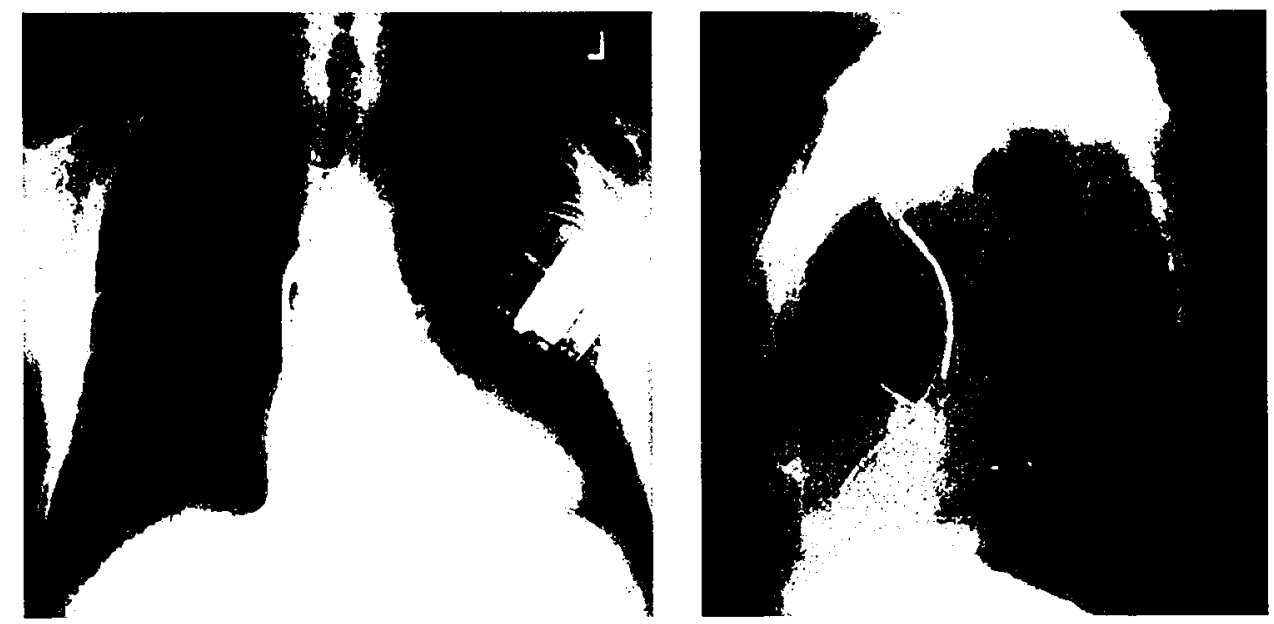

Figure 5. Posteroanterior and lateral chest $X$-ray showing a dual chamber ICD with biventricular pacing capabilities. The high voltage part of the system consists of the RV coil and SVC coil integrated in one tripolar electrode, and the active can. The unipolar left ventricular pacing lead is in one of the branches of the coronary sinus. Pacing is possible separately and simultaneously in both ventricles. The pacing configuration is programmable not only for bradycardia but also for antitachycardia pacing. The sensing dipole remains small, consisting of the tip of the RV lead and the distal part of the RV coil, which is important for ICD sensing. 
A. Unfiltered Endocardial Ventricular Electrogram

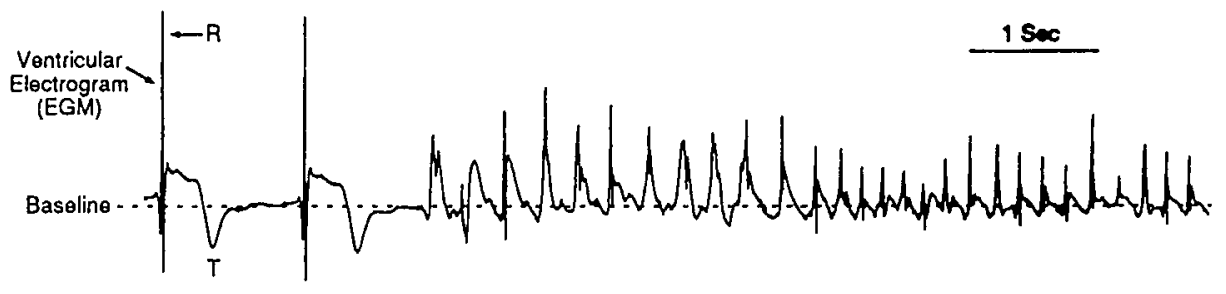

B. Sensing With Automatic Gain Control

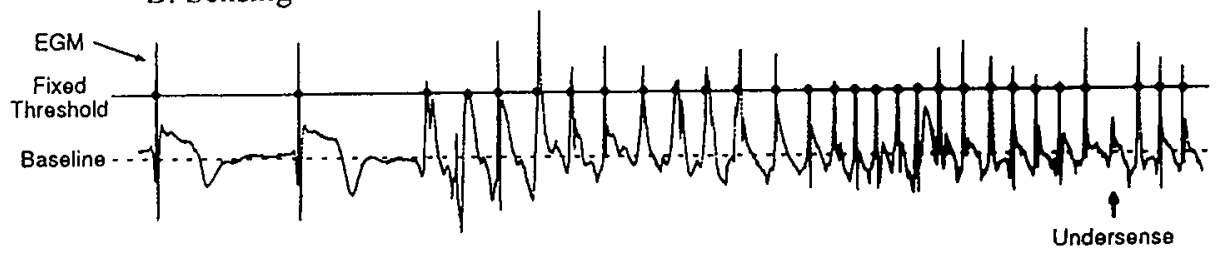

C. Sensing With Automatic Adjusting Threshold

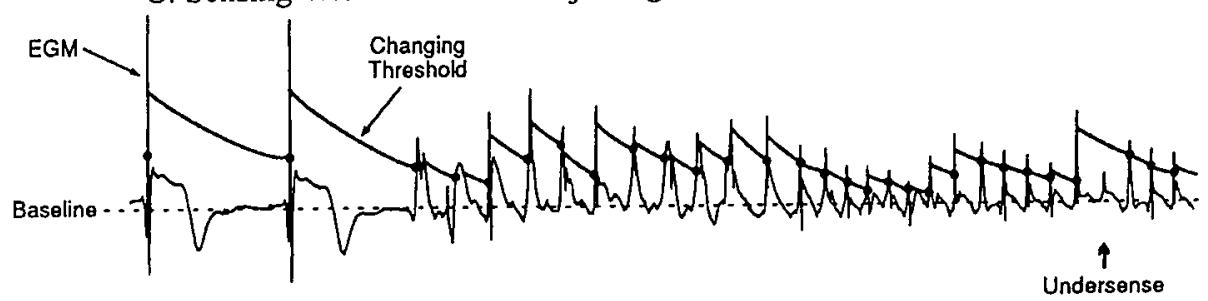

Figure 6. ICD principles of cardiac rhythm sensing. A) Endocardial ventricular electrogram of raw signals during sinus rhythm and ventricular fibrillation. Large differences between the amplitude of the $R$ wave during sinus rhythm and the fibrillatory waves are shown. B) Automatic Gain Control as applied in Guidant devices. The sensing level is fixed and the small fibrillation signals are amplified (with a different gain) compared with those in panel A. Sensed events are marked by dots when the signal crosses the fixed threshold. C) Automatic Adjusting Threshold applied in Medtronic devices. The threshold varies (with different decay) according to the amplitude of the electrogram. Sensed events are marked by dots when the signal crosses the variable threshold.

(24, with permission of W.B. Saunders Company). 
matic gain control, the gain of the signals is adjusted and the threshold remains constant. Auto-adjusting threshold, applied in Medtronic ICDs, uses constant amplification of the amplitude of the (part of the peak) $R$ wave, which becomes the starting amplitude of the time-decaying threshold. It means that, in auto-adjusting threshold, the gain of the signal remains constant and the threshold is adjusted. Both systems have their "attack" time that determines how rapidly the gain or the threshold can increase and decrease. In both systems the bandpass filter should accept near field ventricular (or in the atrium atrial) depolarization signals, while rejecting repolarization or far field signals and extracardiac signals. The bandpass filter is designed to match the frequency spectrum of ventricular (respectively atrial) electrograms. The amplifiers of the signals should have the ability to recover quickly after pacing and shock pulses, reject offsets due to electrode polarization after high voltage therapy and have high signal-to-noise ratios (24).

\section{TACHYARRHYTHMIA DETECTION}

\section{General considerations}

The first generation ICDs had only one detection zone that was not programmable. The ICD model introduced in 1982 added a rate-counting channel to the original AID. However, the rate thresholds were fixed and had to be predetermined before ordering the device from the manufacturer. Noninvasive programmability of two detection zones was not available. From 1993 on ICDs have up to three programmable rate detection zones in which zone-specific detection enhancements and therapies are possible. Detecting ventricular arrhythmias is based on the duration of the cardiac cycle length, which is evaluated on a beat-to-beat basis. The ICD compares each detected ventricular interval with the programmed detection zones. If the cardiac cycle length is within the range of a tachyarrhythmia detection zone, the device marks that cardiac cycle as an arrhythmic event. Each such event increases an event counter in the device memory. For ventricular events within the detection zones, counting and logic algorithms analyze a series of recent cycles, before the type of arrhythmia is classified. When the event counter reaches the programmed number, a tachyarrhythmia episode is declared. ICD detection algorithms for VF are designed to have high sensitivity despite reduced specificity, because the potential consequences of underdetection VF are grave. This approach includes all aspects of the detection process from initial detection to episode termination. Because some undersensing of ventricular fibrillation is possible even with autogain and autothreshold methods, the VF detection counting algorithms must tolerate some degree of undersensing. Initial detection criteria are fulfilled when a certain percentage (typically $70-80 \%$ ) of intervals 


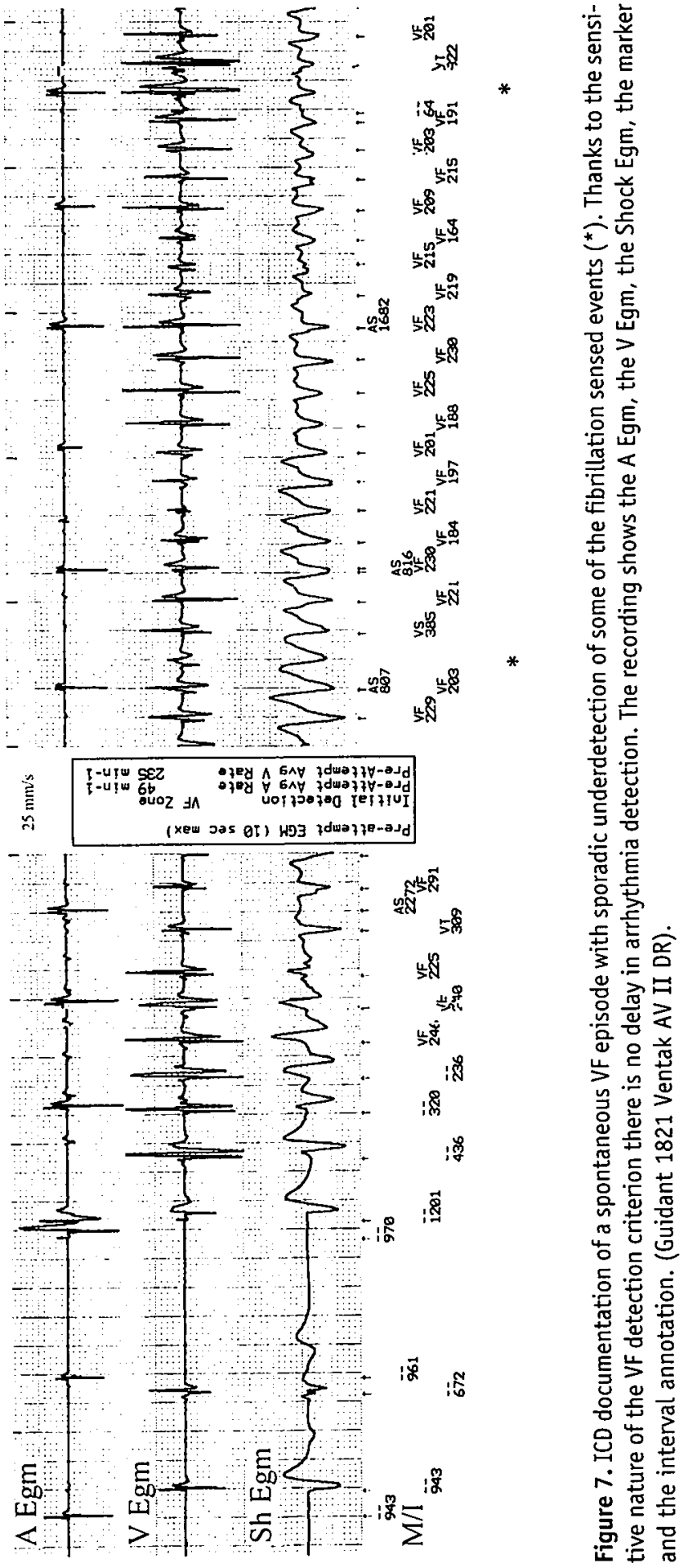


are within the VF zone (fig 7). In contrast, detection algorithms for ventricular tachycardia must balance the risk of underdetection with the consequences of inappropriate detection. A sensitive detection algorithm is appropriate for faster VT (usually having cycle lengths $<300 \mathrm{~ms}$ ), because the consequences of diagnostic mistakes are serious and the probability of rate overlap with supraventricular tachyarrhythmia (SVT) is low. A specific detection algorithm is appropriate for haemodynamically stable VT, because the risk of underdetection is low and the probability of rate zone overlap with SVT is high.

\section{Ventricular arrhythmia detection - basic algorithms}

In Medtronic ICDs ventricular arrhythmia detection is based on number and cycle length of ventricular (VV) intervals (25). To fulfill detection criteria, a programmed number of ventricular intervals must be within the VF or VT zone respectively. For VF detection $75 \%$ of consecutive ventricular intervals must be within the VF zone, for example 18 of 24 or 12 of 16. For VT detection the programmed number of the consecutive ventricular intervals, for example 24 must be within the VT zone. The ICD can be programmed to use the separate classification for fast ventricular tachycardia (FVT). The FVT zone can be coupled to the VF and to the VT zone (fig 8). FVT detected via VF, uses the VF counter, and when the number of intervals reaches the number required for $V F$ detection, the last 8 intervals are reviewed. If any of the last 8 intervals is in the VF zone, the arrhythmia is classified as VF. If all 8 last intervals are outside the VF zone, the arrhythmia is classified as FVT. FVT detected via VT uses the VT counter, and when the number if intervals reach the number required for VT detection, the last 8 intervals are reviewed. If any of the last 8 intervals is in the VF or FVT zone, the arrhythmia is classified as FVT. If all 8 of the last intervals are outside the FVT and VF zone, the arrhythmia is classified as VT. FVT detected via VT is subjected to the same rules of detection specificity (consecutive beats, enhancement criteria) as VT itself. On redetection or after VF detection the FVT zone merges with the VF or the VT zone, depending on programming (fig 9).

When the intervals of ventricular arrhythmia are fluctuating between the VF and VT zones, the Combined Count criterion is applied to avoid excessive detection times. For the ventricular arrhythmias in the VF/VT border-zone the detection time might become excessive, because the increase of the event counter for either the VF or VT zone separately is being interrupted by the event from the other zone. The Combined Count detection starts, when the VF counter reaches six and is satisfied when the sum of the VF and VT events equals the number of $7 / 6$ intervals programmed to detect VF. The last 8 intervals of the Combined Count detection are reviewed. If any of the 8 intervals was in the VF zone, the arrhythmia is classified as VF, if all 8 intervals were 


\section{Ventricular arrhythmia detection zones}
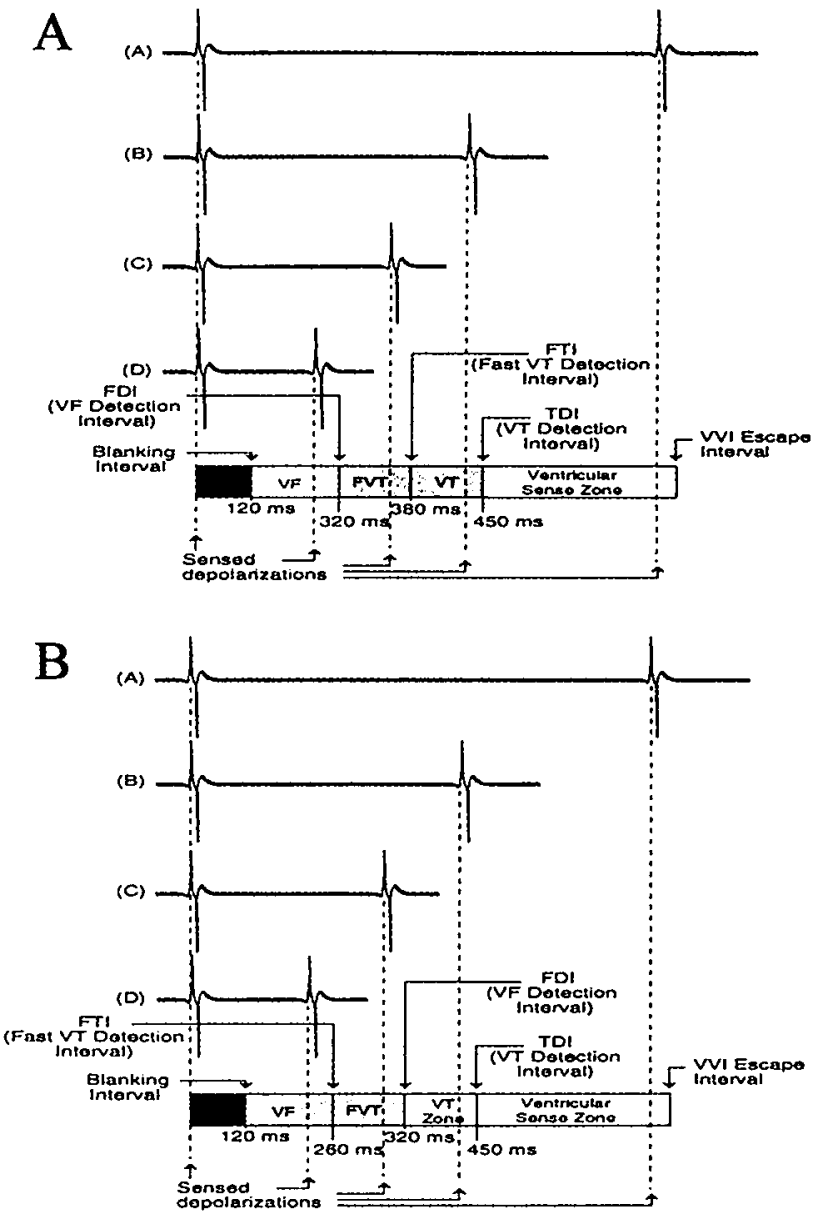

Figure 8. Three detection zones for ventricular tachyarrhythmias (Medtronic ICD). The ventricular blanking period following sensed events is $120 \mathrm{msec}$. Ventricular events outside that $120 \mathrm{msec}$ are classified as arrhythmic if they are within the detection interval of VF, or FVT, or VT. The FVT zone can be connected to the VT zone (A) or the VF zone (B), following the rules of VT or VF detection respectively. Ventricular sensed events with VV intervals longer than the VT intervals are classified as non-arrhythmic. The non-arrhythmic events can be normally sensed events or bradycardia paced events. The paced events occur at the programmed pacing rate and in modern ICDs (with sensor or dual chamber pacing) they can have variable intervals. (25, with permission of Medtronic). 


\section{A Reversion to two zones with FVT detection via VF counting}

\section{Before initlal defection: three detection zones}

\begin{tabular}{|c|c|c|c|c|}
\hline Blanking & VF Detection Zone & FVT Detection Zone & VT Detection Zone & $\begin{array}{c}\text { VVI Escape } \\
\text { Period }\end{array}$ \\
\hline
\end{tabular}

After detection of VF: two zones for redetection

\begin{tabular}{|c|c|c|c|}
\hline Blanking & VF Detection Zone & VT Delection Zone & $\begin{array}{l}\text { WI Escape } \\
\text { Poriod }\end{array}$ \\
\hline ation & & & \\
\hline
\end{tabular}

\section{B Reversion to two zones with FVT detection via VT counting}

Before initial detection: three detection zones

\begin{tabular}{|c|c|c|c|c}
\hline Blanking & VF Detection Zone & FVT Detection Zone & VT Detection Zone & $\begin{array}{c}\text { VVI Escape } \\
\text { Period }\end{array}$ \\
\hline
\end{tabular}

After detection of VF or FVT: two zones for redetection

\begin{tabular}{|c|c|c|c|}
\hline Blanking & VF Detection Zone & FVT Detection Zone & $\begin{array}{c}\text { VVI Escape } \\
\text { Period }\end{array}$ \\
\hline
\end{tabular}

Figure 9. Merging of the three ventricular tachyarrhythmia detection zones (Medtronic ICD). The merged zone uses the event counting and therapies for a faster arrhythmia until episode termination. A) For FVT via VF detection, the FVT zone merges with the VF zone after detection or redetection of VF. B) For FVT via VT detection, the FVT zone merges with VT zone after detection or redetection of FVT or VF. (25, with permission of Medtronic). 


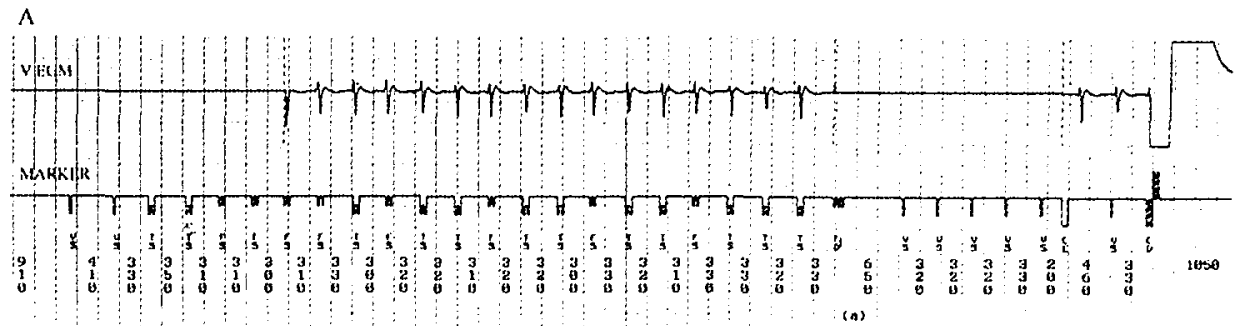

$25 \mathrm{~mm}$ 's

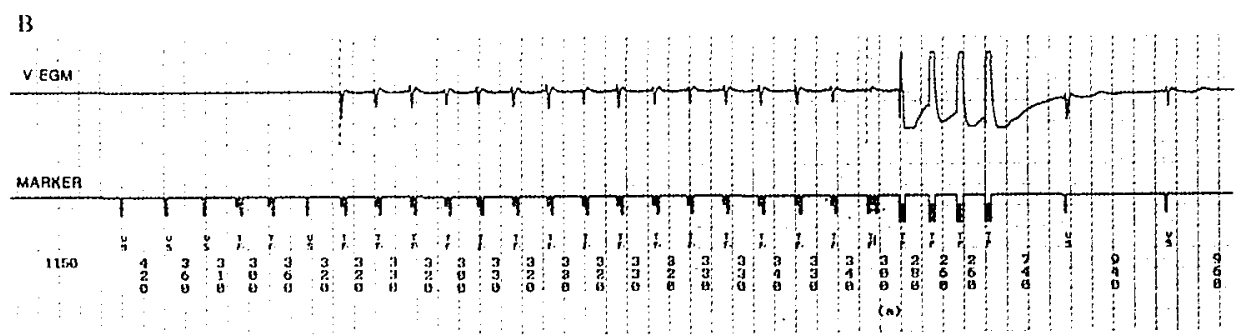

Figure 10. Detection of a spontaneous ventricular arrhythmia having intervals that fluctuate between the programmed VT (16 intervals, $350-320 \mathrm{msec}$ ) and VF (18 of 24 intervals, $\leq 310 \mathrm{msec}$ ) zone using a combination of intervals (A) and zones (B). The ICD rhythm strip shows the V Egm, and the marker channel annotation with interval data. A) Combined Count Detection in the two-zone configuration. Within the Combined Count Detection zone of $(7 / 6 \times 18 \Rightarrow) 21$ intervals, the last eight intervals contain 2 VF events; the episode is classified as VF and treated with a defibrillation shock. B) FVT via VF detection in the three zone configuration in the same patient. The arrhythmic intervals are within the FVT zone (280-340 msec); the episode is classified as FVT and treated successfully with ATP. (Medtronic 7218).

outside the VF zone, the arrhythmia is classified as VT. Combined Count detection also applies to the FVT zone.

Both the Combined Count Criterion and the FVT via VF detection allow safe functioning of the interval-based detection of the ventricular arrhythmia (fig 10). The Combined Count Criterion functions as therapy accelerator, but the last 8 intervals of the Combined Count Detection will decide, whether the applied therapy will be the one of the higher or lower zone (fig 10A). The FVT that can be combined with the VF zone, allows programming of antitachycardia pacing therapies for fast pace-terminable ventricular arrhythmias with a cycle length that may partially overlap some of the cycle lengths of not pace-terminable ventricular arrhythmias (fig 10B).

In Guidant ICDs ventricular arrhythmia detection is based on rate and duration criteria and there are three separate detection zones for VF, FVT and VT (26). For the initial detection of a ventricular arrhythmia, 8 of the 10 most recent ventricular intervals must be within the detection window for VF, FVT or VT. The detection window 


\section{Multizone ventricular arrhythmia detection}

VF Zone RATE $=200 \mathrm{~min}^{-1}=300 \mathrm{~ms}$ $V T$ Zone RATE $=150 \mathrm{~min}^{-1}=400 \mathrm{~ms}$ $S=$ Slow $F=$ Fast

8 of 10 intervats are fast. VF window is satistied

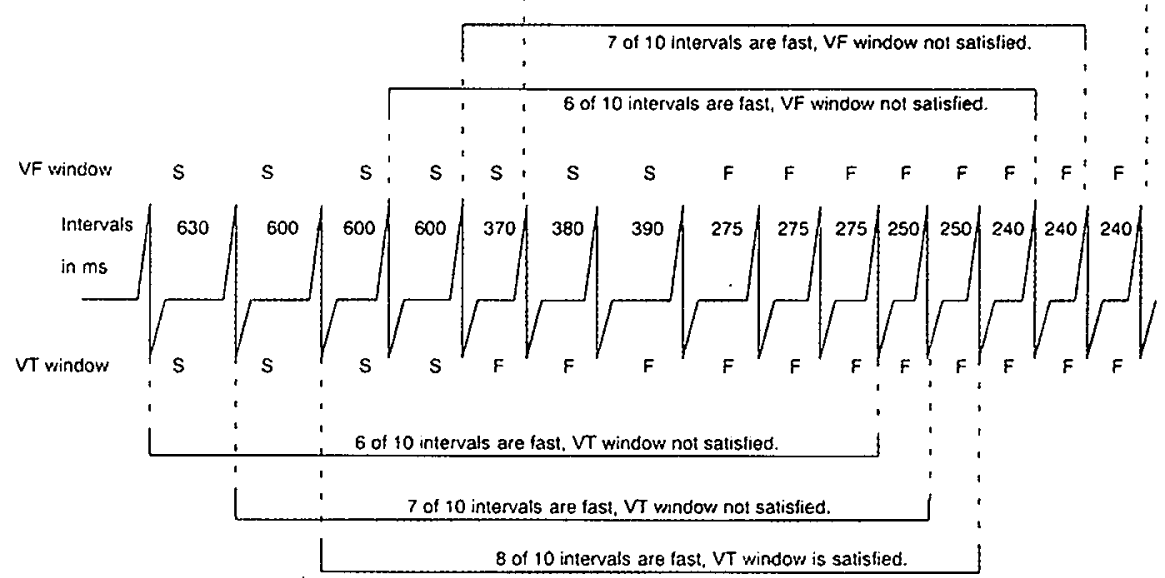

Figure 11. Detection windows for ventricular arrhythmias in a multizone configuration (Guidant ICD). Intervals classified as arrhythmic in a higher zone are also classified as arrhythmic in a lower zone. The initial detection zone determines the duration timer that runs independent of each other within the respective zone. (26, with permission of Guidant).

remains satisfied as long as 6 of the 10 most recent intervals remain within the detection window. After the initial detection has been satisfied, the duration timer is started, during which 6 of the 10 most recent ventricular intervals must satisfy the detection window. In the multizone configuration an interval classified as arrhythmic in a higher zone would also be classified as arrhythmic in a lower zone (fig 11). The duration timers run independently for each of the programmed detection zones, also when the intervals of the ventricular arrhythmia are fluctuating among the zones. The duration timer of the higher zone in which an arrhythmia was initially detected takes priority over the lower zone timer. If the higher zone duration timer expires and detection is still met, therapy for that zone will be initiated. However, if the higher zone detection window does not remain satisfied, the lower zone duration timer takes over and programmed therapy for the lower zone will be initiated (fig 12). 
A

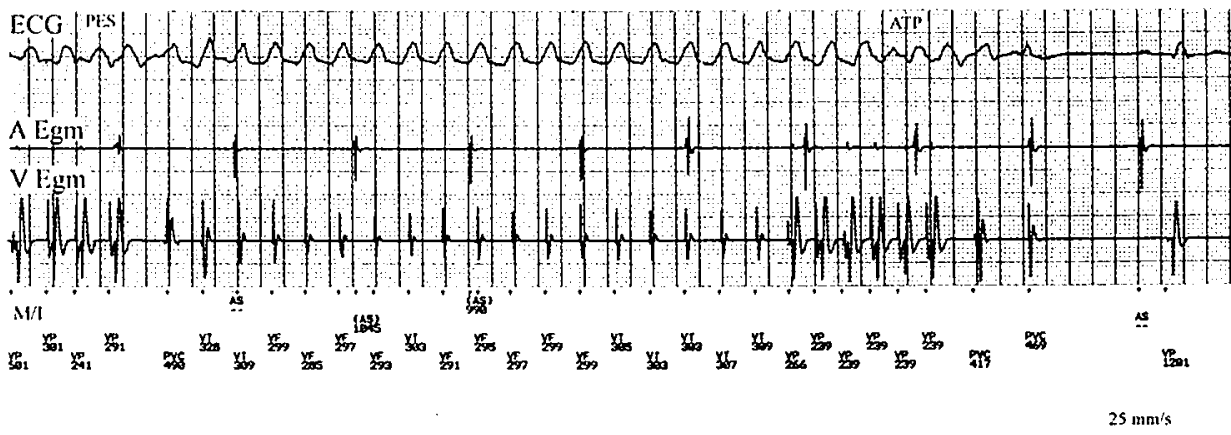

B

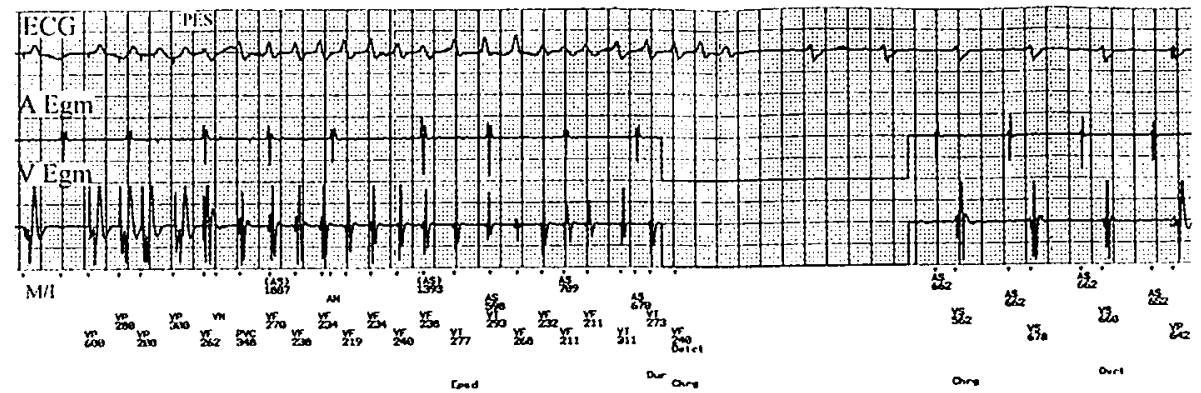

Figure 12. Multizone detection of ventricular arrhythmias induced by programmed electrical stimulation during predischarge testing. The rhythm registration shows a single lead ECG, the A Egm, the $\checkmark$ Egm and the marker channel with interval data. A) The cycle length of the ventricular arrhythmia fluctuates between the VT and VF zone, but the initial detection ( 8 of 10 arrhythmic events) is first met in the VT zone and remains satisfied ( 6 of 10 arrhythmic events) in VT zone before the duration timer expires. The therapy for the VT zone - ATP, terminates the arrhythmia. B) The cycle length of the ventricular arrhythmia fluctuates between the VF and VT zone, but the initial detection ( 8 of 10 arrhythmic events) is first met in the VF zone and remains satisfied (6 of 10 arrhythmic events) before the duration timer expires. The therapy for the VF zone was going to be delivered, however, the arrhythmia terminates spontaneously during charging. After the spontaneous conversion had been confirmed by three non-arrhythmic events following the end of charging, the defibrillation shock is diverted. (Guidant 1831 Ventak AV III DR). 


\section{Detection enhancements}

Detection enhancements were developed in early nineties to improve detection specificity of the single chamber ICDs, but some of the enhancements are also used in the dual chamber devices $(25,26)$. Several enhancements were designed to allow rejection of SVT and prevent inappropriate therapies by the ICD. Single chamber detection enhancements can be programmed only in the lowest detection zones and are meant to be used within heart rates that correspond to haemodynamically stable ventricular arrhythmias.

\section{Rate Stability}

The Rate Stability algorithms are meant to reject atrial fibrillation (AF) as an arrhythmia triggering inappropriate therapy. When enabled, the Rate Stability is continuously being evaluated, as long as the rate criterion for the VT zone remains satisfied.

In Medtronic ICDs the stability criterion starts to be applied when the VT event count reaches three and there is a programmable value (in $\mathrm{msec}$ ) of stability interval available. A ventricular interval is considered instable, if the difference between it and any of the three previous intervals is greater than the programmed stability interval. When an interval in the VT zone fails to meet the stability option, it is marked as a normal (non-arrhythmic) sensed event and the VT count resets to zero (fig 13). This resetting of the VT counter must occur often enough to avoid inappropriate detection.

In the Guidant ICDs the differences in ventricular intervals are calculated on a beat-to-beat basis throughout the duration of the arrhythmia. A current average variance of $R R$ intervals is computed in which the more recent intervals are weighted more heavily than the earlier ones. If the cycle length variability is greater than the programmed value, the rhythm is declared unstable. In the Guidant ICD the rate stability can function as therapy inhibitor and therapy accelerator. In the lowest detection zone Rate Stability functions as the therapy inhibitor, when the differentiation is required between AF with a fast ventricular rate and VT. In the FVT detection zone Rate Stability can function as the therapy accelerator, when the differentiation between monomorphic and polymorphic VT is required. In monomorphic VTs the programmed ATP therapies are applied, in polymorphic VTs the pace sequences are skipped and therapy is accelerated to the first cardioversion shock.

Programming considerations for the Stability Criterion include concern for both VT underdetection and inappropriate SVT detection, in spite of using the algorithm. The underdetection of VT is the result of spontaneous cycle length variations during the arrhythmia. This can occur for example at VT onset, when the onset is preceded by many ventricular premature beats or bouts of non-sustained VTs. On the other hand inappropriate SVT detection can occur when the ventricular rate during atrial 


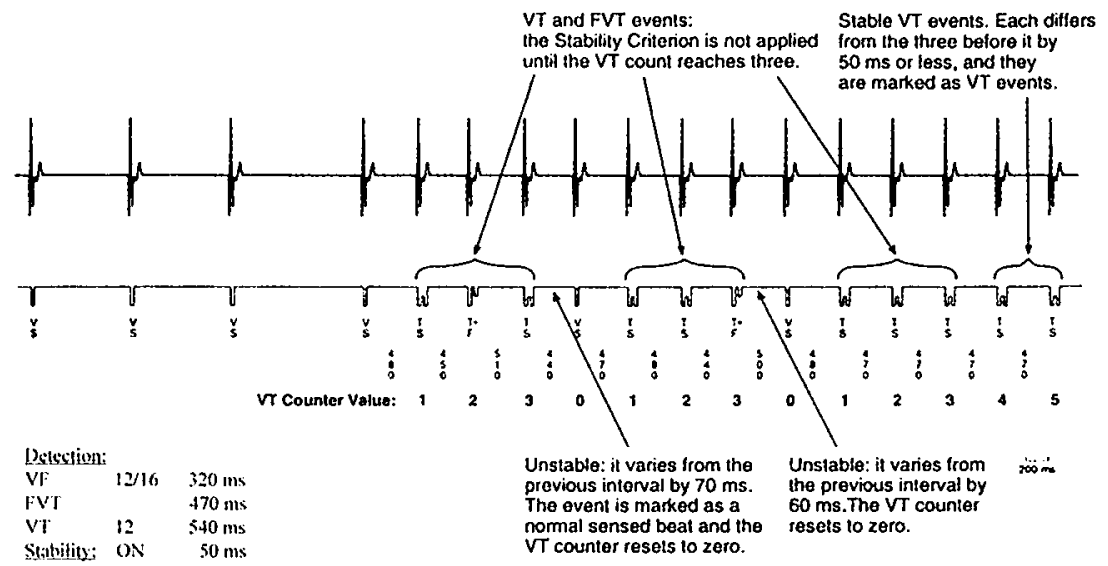

Figure 13. Detection enhancement. Rate Stability Criterion as defined in Medtronic ICD. The ventricular rhythm in the VT zone is considered instable if the difference between a given interval and any of the three previous intervals is greater than the programmed VT Stability interval. (25, with permission of Medtronic).

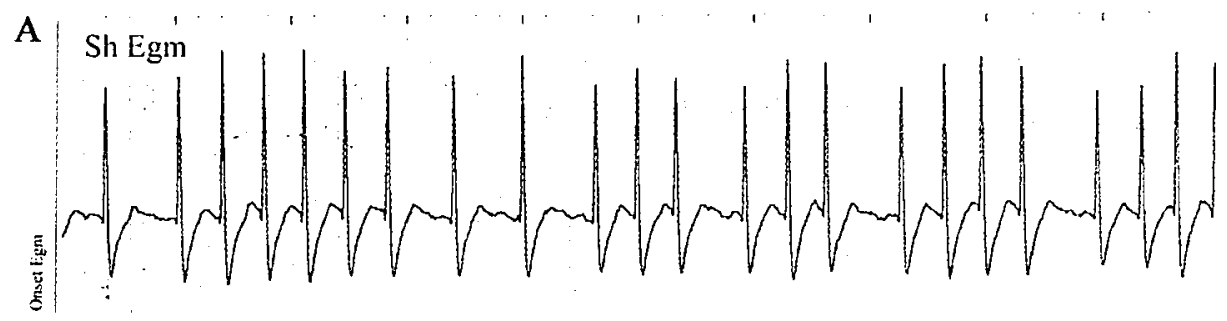

$25 \mathrm{~mm} / \mathrm{s}$

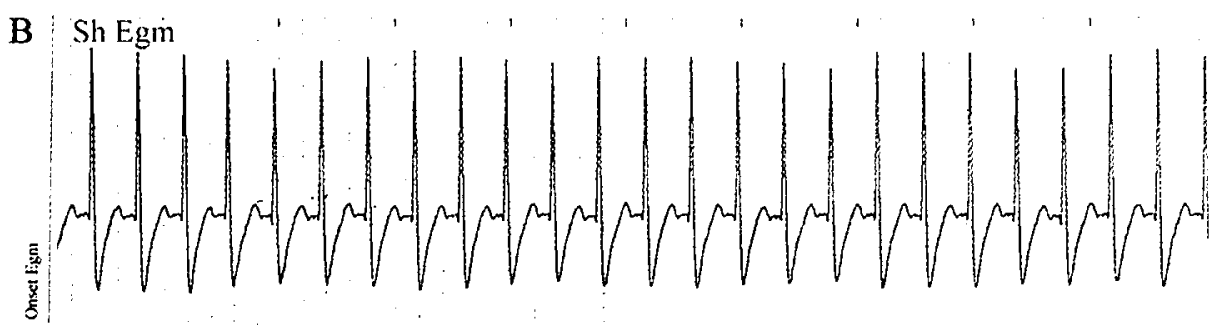

Figure 14. Shortcomings of the single chamber detection enhancements. The Rate Stability. The ICD rhythm strips show the Shock Egm of two spontaneous arrhythmias detected in the VT zone. The Rate Stability prevents inappropriate detection due to AF with a ventricular rate within the VT zone (A). The algorithm fails to withhold inappropriate detection when the ventricular rate during atrial arrhythmia becomes regular (B). (Guidant 1763 Mini II). 
arrhythmias becomes regular, for example when a regular ventricular rate is present during an atrial tachyarrhythmia, such as in atrial flutter (AFL); or when the ventricular rate during $A F$ is very fast and tends to regularize (fig 14).

\section{Sudden Onset}

The Sudden Onset algorithm was designed to prevent sinus tachycardia (ST) as an arrhythmia triggering inappropriate therapy. When enabled it is evaluated only at the moment the ventricular rate enters the VT zone.

In Medtronic ICDs there is a programmable onset percentage describing the abruptness of arrhythmia initiation. An arrhythmia onset is considered to be sudden, if the average of the most recent 4 intervals is shorter, by the programmable onset percentage value, than the average of the 4 intervals that preceded the most recent intervals. If the arrhythmia is in the VT zone, but the onset criterion not satisfied, the ventricular intervals are marked as normal (non-arrhythmic) sensed events (fig 15).

In the Guidant ICDs the criterion can be programmed as a percentage of the cycle length, or as a time interval (in $\mathrm{msec}$ ). The selected onset represents the minimum difference that must exist between adjacent intervals for the five intervals that are below and above the lowest programmed rate threshold. The device measures the intervals prior to the start of the arrhythmia and locates the pair of intervals, where the cycle length changed the most. Thereafter it compares those pivot intervals to any other pair after arrhythmia detection. If the change of cycle length is greater then the programmed onset threshold, the arrhythmia onset is considered sudden; if not, the onset is gradual.

Programming considerations for the Sudden Onset algorithm include both underdetection of VT and inappropriate SVT detection in spite of using the algorithm. Underdetection of VT can occur when the arrhythmia onset cannot be declared abrupt, because the VT occurs during sinus tachycardia or the onset is abrupt but the sinus tachycardia was already within the VT zone, or there is spontaneous acceleration on VT initiation. On the other hand, inappropriate SVT detection can occur because many supraventricular arrhythmias show sudden onset as well (fig 16).

\section{The EGM Width Criterion}

The EGM Width Criterion available since 1994 in Medtronic ICDs was designed to reject narrow $Q R S$ tachycardias as arrhythmias triggering ICD therapy. This detection enhancement uses information on morphology of intracardiac ventricular signals using the far field $\mathrm{R}$ wave EGM. This is in contrast to the EGM normally recorded from the tip-ring bipole of the sensing lead that registers primarily local activation. The far field EGM can be obtained using different parts of the ICD hardware: the can and the $R V$ coil $(A \rightarrow B)$, can and $R V$ ring $(A \rightarrow P+), R V$ coil and $R V$ tip $(B \rightarrow P-)$. Before the algorithm can be applied the available EGM sources and the QRS width obtained using these sources are evaluated for their discriminative abilities to consider the QRS 


\section{Sudden Onset Criterion}

$\begin{array}{lll}\text { Detection: } & & \\ \text { VF } & 12 / 16 & 320 \mathrm{~ms} \\ \text { FVT } & \text { OFF } & \\ \text { VT } & 12 & 500 \mathrm{~ms} \\ \text { Onset: } & \text { ON } & 81 \%\end{array}$
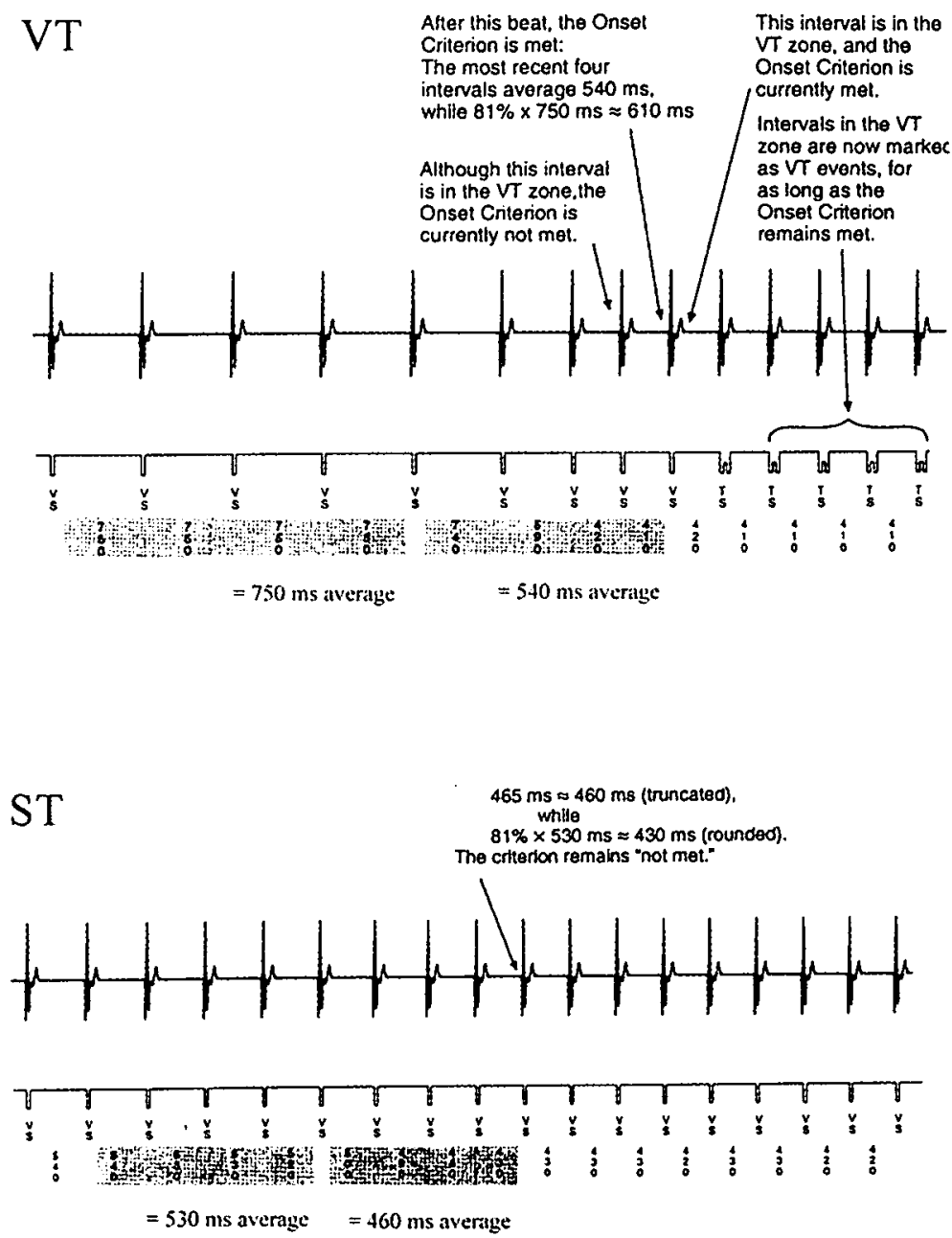

xom

Figure 15. Detection enhancements. Sudden Onset Criterion as defined in Medtronic ICD. A ventricular rhythm that enters the VT detection zone is considered to have a sudden onset, if the average of the last four sensed events is less than the programmed percentage of the average of the previous four sensed events. (25, with permission of Medtronic). 


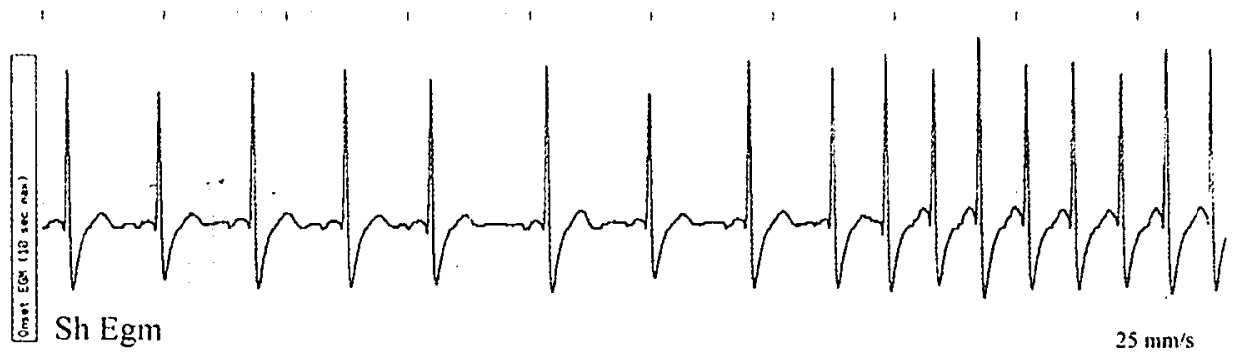

Figure 16. Shortcomings of the single chamber detection enhancements. Sudden 0nset and Rate Stability. The ICD rhythm strip shows the Shock Egm of a spontaneous arrhythmia detected in the VT zone. The Sudden Onset which is meant to discriminate between sinus tachycardia and VT, fails to withhold inappropriate detection, because the SVT shows sudden onset as well. This arrhythmia cannot be rejected based on Rate Stability either. (Guidant 1763 Mini II).

during sinus rhythm as "narrow" and during VT as "wide". Extensive measurements of the QRS widths and slew threshold parameters during sinus rhythm and during VTs, both induced and spontaneous, are required to achieve this goal.

The width is measured using the slew threshold to determine the beginning and the end of the QRS complex. Lower slew thresholds cause greater variations in the QRS measurements during the same rhythm. Higher slew threshold causes more of the initial and late portion of the QRS complex is being excluded from width analysis, which might result in ventricular beats having a similar width as the supraventricular ones.

When the initial VT detection is completed, the device looks back and classifies the last 8 intervals. If 6 or more ventricular events are wide, the criterion is met and the arrhythmia is classified as ventricular. If at least 3 ventricular events are narrow, the criterion is not met and the VT counter is reset to zero. The EGM Width Criterion does not effect the marker channel annotations during the episode, and can be used as active or passive detection enhancement.

Programming considerations for the use of the QRS Width Criterion include the VT underdetection and inappropriate SVT detection in spite of using the algorithm. The underdetection of VT can occur in patients with multiple morphologies of VT, bundle branch reentry VTs or otherwise VTs with morphology resembling a normally conducted supraventricular rhythm. On the other hand inappropriate SVT detection can still occur using this algorithm due to different causes of QRS variability, such as aberrant conduction, higher heart rates, or even a change in the body position.

In some patients their VT may present a problem for the QRS width algorithm. For example patients with more than one VT morphology (fig 17), or patients showing pleomorphic VT morphology within one arrhythmia episode (fig 18). Furthermore, 

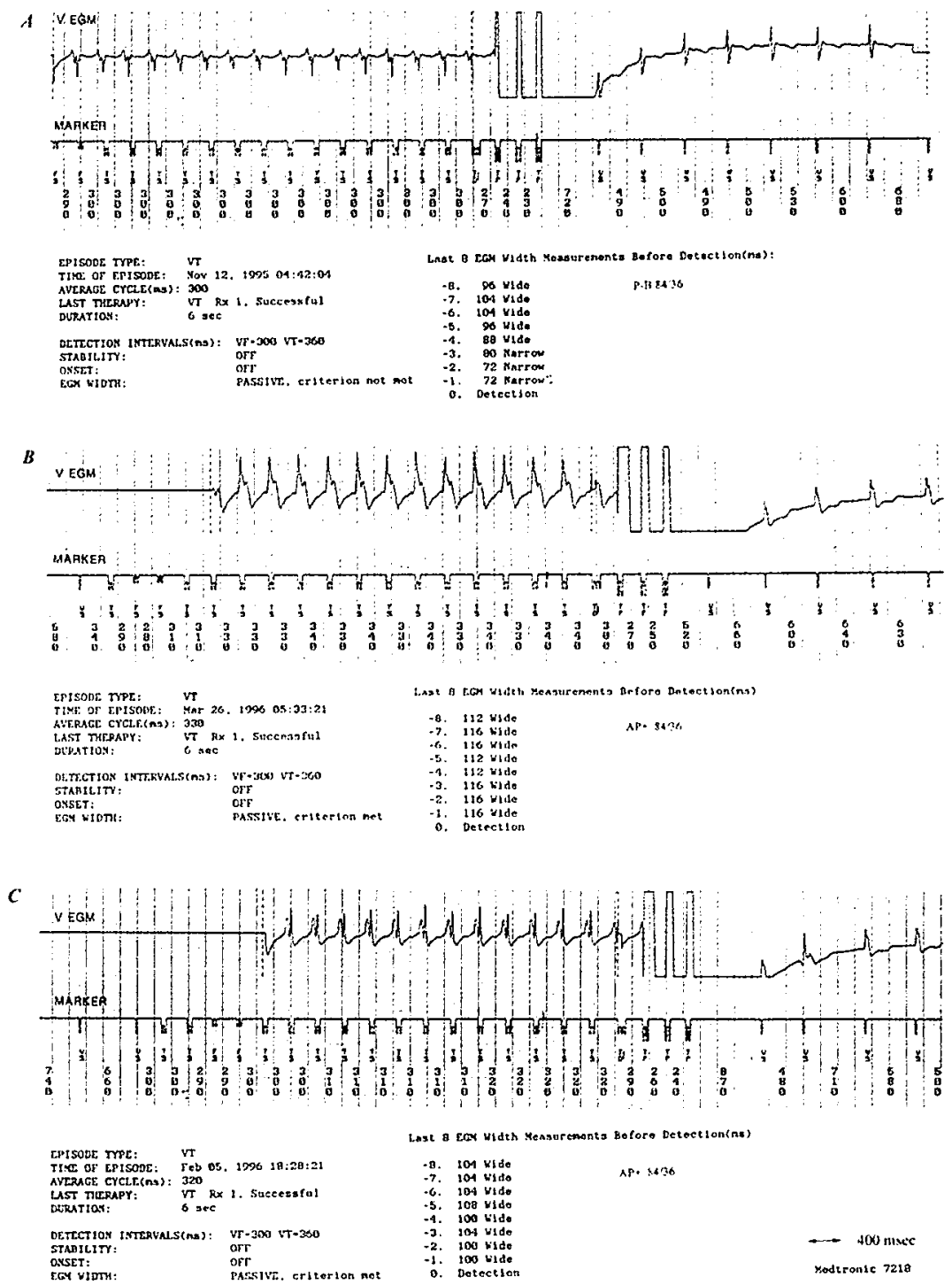

Figure 17. Detection enhancements. QRS width criterion in the setting of multiple VT morphologies. The ICD rhythm recordings of the wideband V Egm, the marker channel annotation and interval data of three spontaneous VT episodes in one patient. The episode text and QRS width measurements for the three VTs are included. Different morphologies of spontaneous VTs occurred in a patient, in whom only one clinical morphology was documented before ICD implantation. The QRS width parameters were programmed based on measurements on predischarge testing and included: the wideband EGM source "P- $\rightarrow B$ ", QRS width for sinus rhythm of $84 \mathrm{msec}$ and the QRS slew threshold of $36 \mathrm{msec}$. Using these parameters the VT would have been underdetected, because three beats are measured by the device as "narrow" (A). After the EGM source had been changed to " $A \rightarrow P_{+}$" the detection enhancement was satisfied in recurrent VT episodes, even though more morphologies of VT were registered $(B, C)$. (Medtronic 7218 ). 
$\wedge$

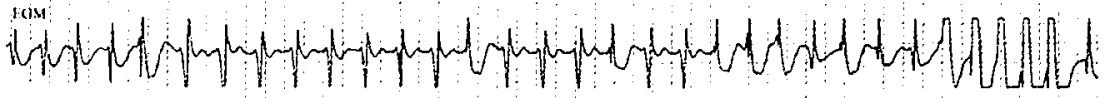
minken

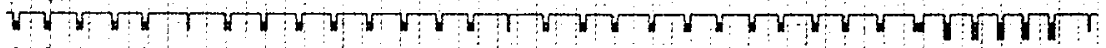

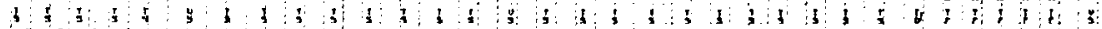

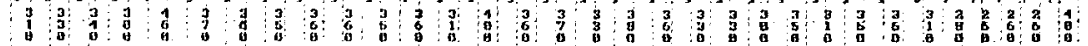

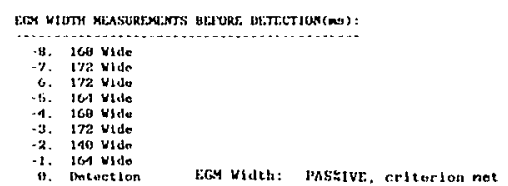

B

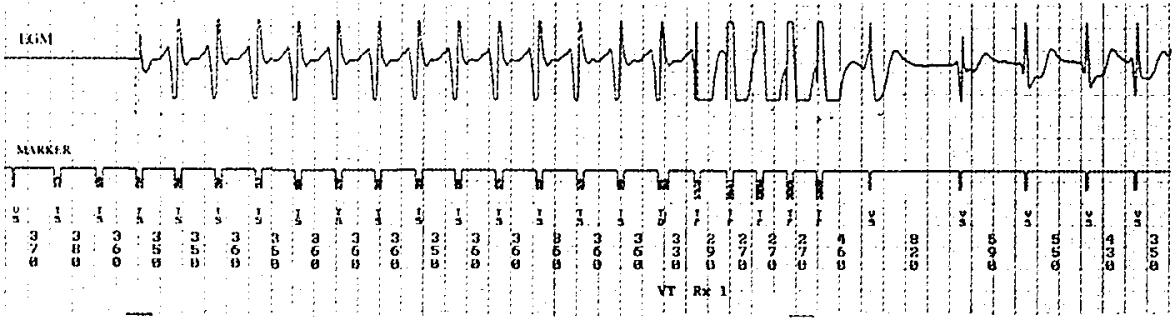

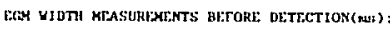

-8.160 Wide
$-7 . \quad 194$ Vido

-7. 1.14 Wide

6.104 wide

4. 119 wido

-4. 14U Wide
$-3 . \quad 161$ wide

2. 160 vide

t. 136 bid

o. Dotoction Ber ytdth: paseivr, criturlon inet

c

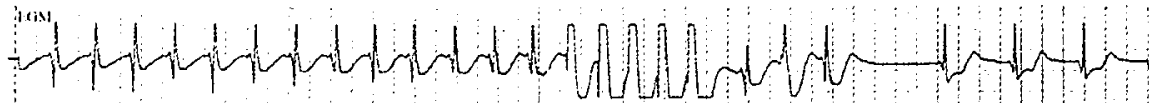

MARKEA

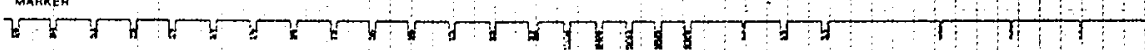

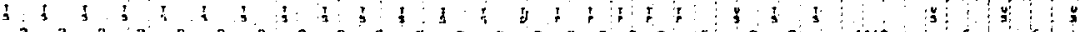

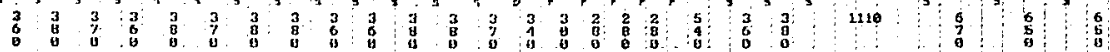
is 1 .

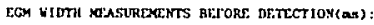

-8. 92 vido

$\begin{array}{lll}-7 . & 92 \\ -6 . & 92 & \text { vide }\end{array}$

-5. 68 vide

-4. 108 vido

3. 80 uldo

92 vidio

Datoction EGM with: PAssive. criturion not

\section{DETECTION SETTINGS}

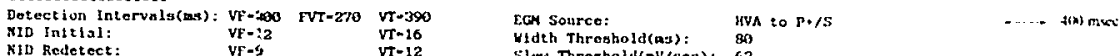

For legend, see next page. 
Figure 18. Detection enhancement. QRS width criterion in the setting of pleomorphic VT. The ICD rhythm recordings of the wideband $V$ Egm, the marker channel annotation and interval data of three spontaneous VT episodes in one patient. The episode text and the QRS width measurements for the three VTs are included. The QRS width of $80 \mathrm{msec}$ and QRS slew threshold of $63 \mathrm{msec}$ were programmed to discriminate conducted sinus beats and VT. A) During a spontaneous VT episode that occurred on exercise three QRS morphologies, with fusion between some beats were observed, as well as considerable variations in arrhythmia cycle length. Using the $A \rightarrow P+$ as the Egm source all QRS complexes were classified as "wide" and could easily be discriminated from the conducted sinus beats. More ATP attempts were necessary to terminate the VT, the second tier of Ramp + caused acceleration into the FVT zone, in which the VT could still be stopped with pace therapy. B) A spontaneous VT episode occurring at rest in the same patient. The VT (B) shows only one of the QRS morphologies, which were registered in VT (A) and less variation in cycle lengths. The detection criterion can still easily be met, as far as the QRS width is concerned and the VT (B) is terminated by the first attempt of ATP. C) A spontaneous VT episode occurring also at rest in the same patient. The VT (C) shows QRS morphology present as one of the morphologies registered in VT (A) with normal variation in the cycle length. This time the programmed QRS width detection criterion is just satisfied, with the values during VT only 8-12 msec more than the ones programmed for conducted sinus rhythm. Of note, although the VT (C) morphology was also observed in a number of beats during the VT $(A)$, none of the beats in VT (A) on exercise were as narrow as in VT (C). (Medtronic 7218).

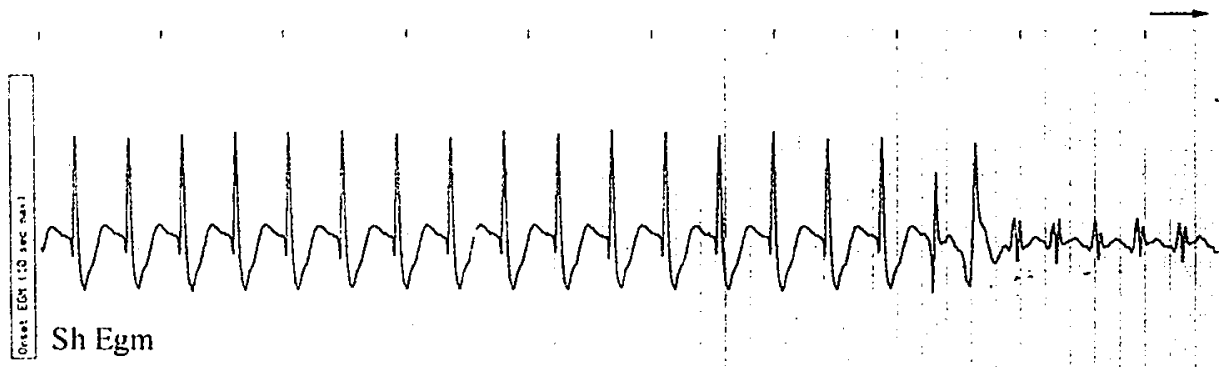

$25 \mathrm{~mm} / \mathrm{s}$

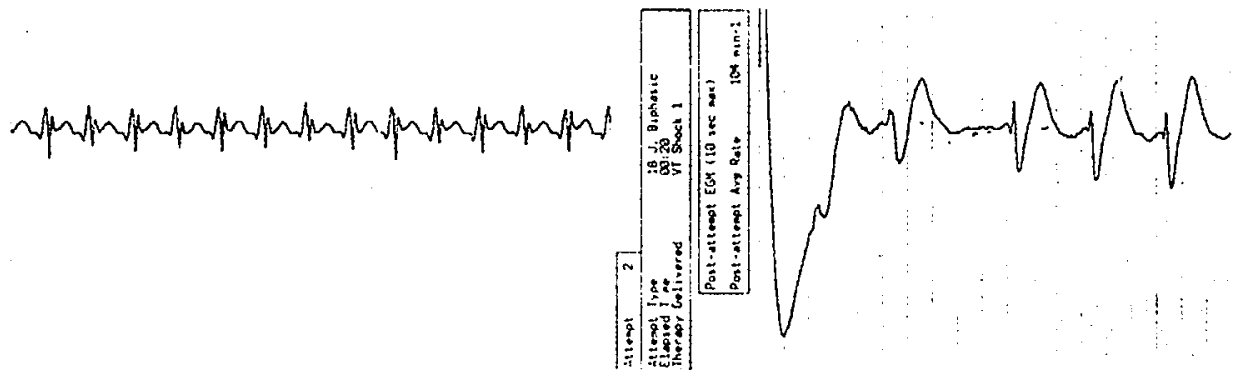

Figure 19. Shortcomings of the single chamber detection enhancement. The wide band shock Egm recording was obtained using the (RV coil $+\mathrm{SVC})$ - the (Can) as a dipole. The shock Egm recording of an arrhythmia detected in the VT zone shows three morphologies of QRS. Presumably the first is a supraventricular tachycardia in the VT zone, the second the VT itself, the third a supraventricular rhythm with aberration after the cardioversion shock. Alternatively, there might be two morphologies of VT in the upper panel. Except comparing the Egm morphologies recorded during this arrhythmia episode with the Egm morphology registered during sinus rhythm in this patient, no other diagnostic tools are available to support the presumption. (Guidant $1763 \mathrm{Mini}$ II). 
when the VTs cannot be evaluated, because they could not be induced during predischarge testing or new VTs appear later in the history of the heart disease.

In Guidant ICDs the wideband ventricular Egm, the Shock Egm, is recorded using the dipole RV (+SVC) - Can and has no programmable parameters. The Shock Egm is not involved in device based arrhythmia discrimination, but can be recorded as stored Egm in detected ventricular arrhythmia episodes (fig 19).

\section{Dual chamber arrhythmia detection}

Dual chamber detection algorithms are available since 1995 using information on intervals and the relation between the atrial and the ventricular events to discriminate between ventricular and supraventricular arrhythmias.

The first dual chamber arrhythmia detection algorithm PARAD (ELA Medical) uses the classical ventricular rate criteria for initial zones delineation and employs the VT: SVT discrimination criteria within the programmed VT zone (27). Arrhythmia discrimination is based on the analysis of four criteria: RR stability, AV association, onset and chamber where rhythm acceleration originates. The ventricular rhythm is considered stable if the dispersion of the $R R$ intervals within the detection window is low. In arrhythmias with stable RR intervals, the AV association is further analyzed. The atrial and ventricular rhythm are associated if the PR intervals are stable and the level of association can be in a 1:1 (sinus and atrial tachycardia), $>1: 1$ (atrial flutter) or $<1: 1$ (VT) manner. There is no AV association if the PR intervals are variable. In arrhythmias with 1:1 association the arrhythmia onset is further analyzed. The onset can be sudden or gradual. In arrhythmias with sudden onset the chamber of acceleration is analyzed and can be the atrium or the ventricle. The initial experience of the PARAD reported $94 \%$ specificity of VT: SVT discrimination ability (28). Later considerable numbers of atrial sensing-related errors were observed that were responsible for inappropriate arrhythmia diagnosis (29).

The Medtronic dual chamber PR Logic algorithm uses information about the rate, the regularity, the pattern and the association of atrial and ventricular events $(30,31)$. The pattern recognition applies a couple code syntax that analyses the number and the timing of the $P$ waves within consecutive $R R$ intervals. At the same time the pattern recognition takes measures to reject sensing phenomena that might erroneously influence the detection and discrimination process, such as far field $\mathrm{R}$ wave sensing on the atrial channel, or ventricular and atrial premature beats. Arrhythmias detected within the VT or VF zone are subjected to a number of diagnostic steps after which the PR Logic classifies an arrhythmia as ventricular, supraventricular (fig 20) or dual (fig 21). The range of tachyarrhythmia cycle lengths, in which the PR Logic can operate, is restricted by the programmable SVT limit, but it can overlap part of the VF detection zone. 
A

- - ST

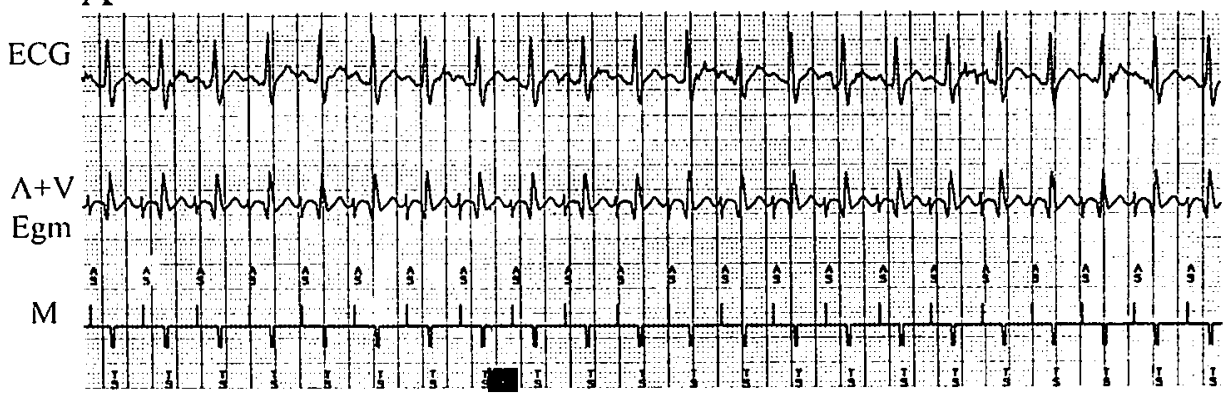

B

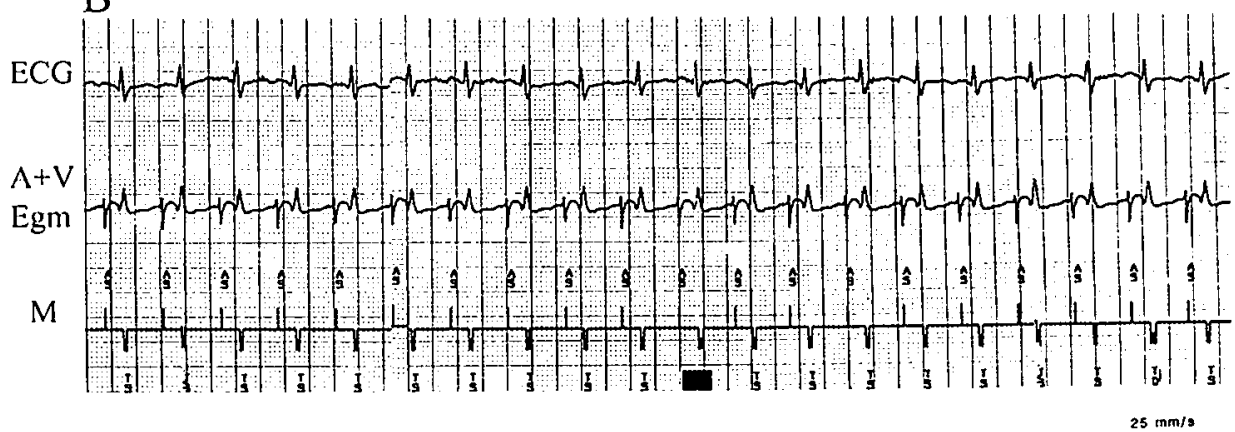

Figure 20. Dual chamber arrhythmia detection using the PR Logic algorithm. An example of sinus tachycardia in the VT zone. The registration shows a single lead ECG, the wideband $A+V$ Egm and the marker channel annotation. A) The dual chamber VT: SVT discrimination rules diagnose correctly sinus tachycardia occurring in the VT detection zone. B) When the dual chamber detection is switched off, the single chamber detection algorithm diagnoses a ventricular arrhythmia, as soon as the ventricular rate enters the VT detection zone during the programmed number of intervals. (Medtronic 7250 Jewel AF).
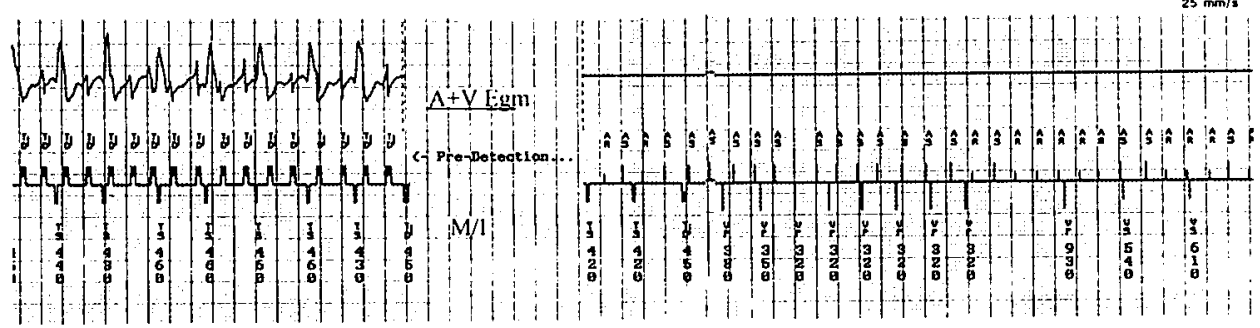

Figure 21. Dual chamber arrhythmia detection using the PR Logic algorithm. An example of a dual arrhythmia, VT and AT occurring simultaneously. The ICD rhythm recording of the wideband $A+V$ $\mathrm{Egm}$, the marker channel annotation with the ventricular interval data. Shown is a spontaneous VT episode occurring during the atrial arrhythmia. Following pace-termination of VT, the atrial arrhythmia continues. (Medtronic 7250 Jewel AF). 
In the first Medtronic model of the dual chamber ICD, atrial arrhythmia detection received special attention, because of its importance not only for the VT: SVT rejection rules, but also for atrial arrhythmia management (30). Atrial therapies, mode switching, pacing algorithms for prevention of atrial arrhythmias, are all dependable on adequate detection of the atrial rhythm. The device has two separate detection zones for atrial fibrillation (AF) and atrial tachycardia (AT), the median atrial rate being the arrhythmia classifier. It is possible to program an overlap zone in which the atrial arrhythmia is classified as AF or AT depending on atrial rate regularity.

The Guidant dual chamber arrhythmia detection and discrimination algorithm combines the information on the atrial and the ventricular rhythm with the single chamber detection enhancements (26). Just as other detection enhancements, the dual chamber VT:SVT rejection rules are available only within the lowest detection zone. The Atrial Fibrillation Rate Threshold enhancement compares the atrial rate to the programmed threshold and is always used in conjunction with the ventricular stability algorithm. If the atrial rate is faster than the AF Rate Threshold and the ventricular rate is unstable, the algorithms diagnoses AF with fast ventricular rate and VT therapy can be withheld. The atrial rate is above the AF Rate Threshold, when 6 of the 10 most recent atrial intervals are faster than the programmed threshold value and continues to be satisfied as long as 4 of the 10 atrial intervals are faster that the threshold. When enabled the stability algorithm is activated.

The safe use of the detection enhancements that are to inhibit inappropriate therapy in the Guidant ICD is possible thanks to the option of inhibitor override. The inhibitor override protects against the possibility of delayed detection or even underdetection of VT that might occur due to application of other than rate only detection criteria. The inhibitor override bypasses the detection enhancement, when the presence of true VT, and not SVT, becomes very suggestive.

The $\mathrm{V}>\mathrm{A}$ algorithm is an inhibitor override that uses the relationship between atrial and ventricular rate to overrule either or both sudden onset and rate stability inhibitors. The $V>A$ is fulfilled, when the average ventricular rate exceeds the average atrial rate by more than 10 beats/minute (fig 22). The V $>A$ algorithm can be used only in patients with no 1:1 VA conduction during the VT. Therefore, the presence and consistency of retrograde conduction need to be tested on more occasions and under different circumstances, before the $V>A$ can safely function to overrule therapy inhibitors (fig 23, fig 24).

The Sustained Rate Duration time enables to overrule the therapy inhibitors, if the fast ventricular rate in the VT zone continues for longer than a programmable period of time. This is an additional safety measure that protects against potentially prolonged underdetection of VT.

The SMART Detection (Biotronic) algorithm discriminates between VT en SVT using the following criteria: atrial and ventricular rate; rate stability; multiplicity- the 

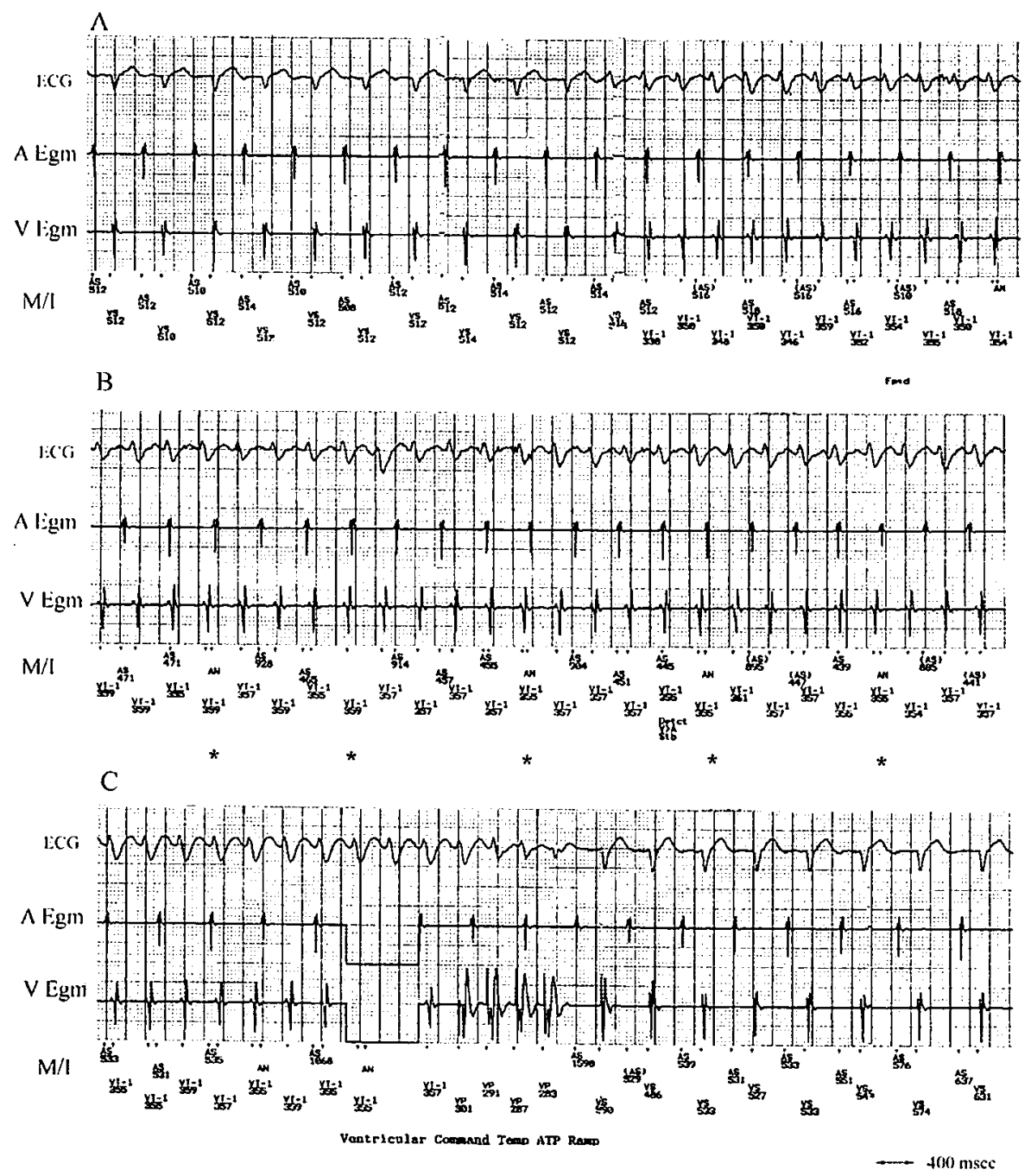

Figure 22. Dual chamber arrhythmia detection using the Guidant detection algorithm. The registration shows a single lead ECG, the A Egm, the V Egm, the marker channel and the interval annotation. The three panels show the initiation, the detection and the termination of a spontaneous VT episode. A) The VT is initiated during sinus tachycardia. The single chamber detection enhancements are fulfilled during initial detection - the arrhythmia has a sudden onset and is stable. B) At the moment of detection the VT CL is $360 \mathrm{msec}$ and the sinustachycardie $\mathrm{CL} 440 \mathrm{msec}$. Although the ventricular rate is indeed higher than the atrial rate, the $V>A$ algorithm is satisfied based on false measurements in this case. Due to the cross chamber post-ventricular atrial blanking ( $80 \mathrm{msec}$ ), approximally $25 \%$ of the atrial sensed events are undersensed, resulting in atrial intervals being longer in the marker channel than indicated by the A Egm. This happens particularly when both the ventricular rate and the atrial rhythm have similar and fast rates. In that case many of the atrial events are in the "vicinity" of the ventricular events, having a chance of being blanked. C) The sinus tachycardia decelerates, but paradoxically has a higher average rate than in panel $B$ because less atrial events are being blanked. The commanded ATP terminates the VT, the sinus tachycardia continues. (Guidant Ventak 1821 AV II DR). 
$\Lambda$

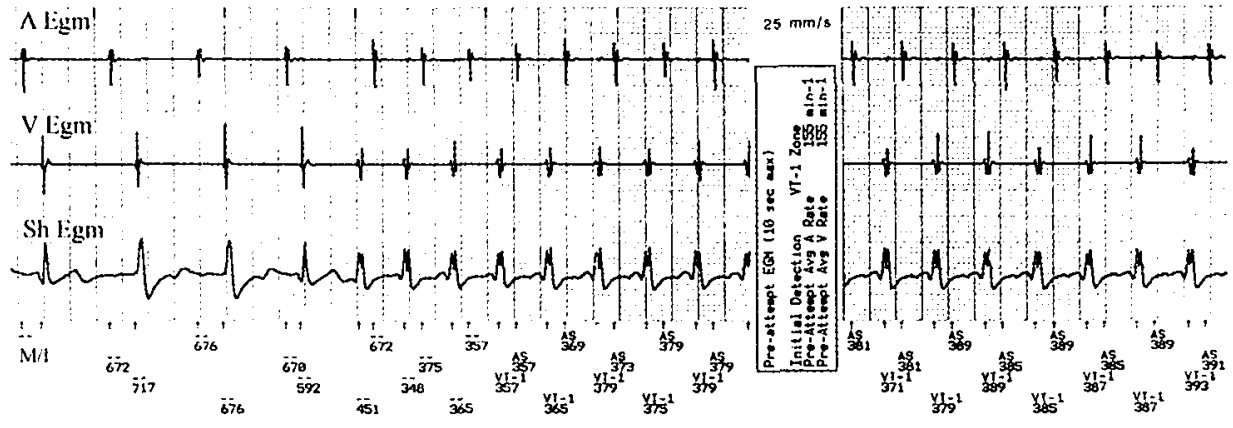

B

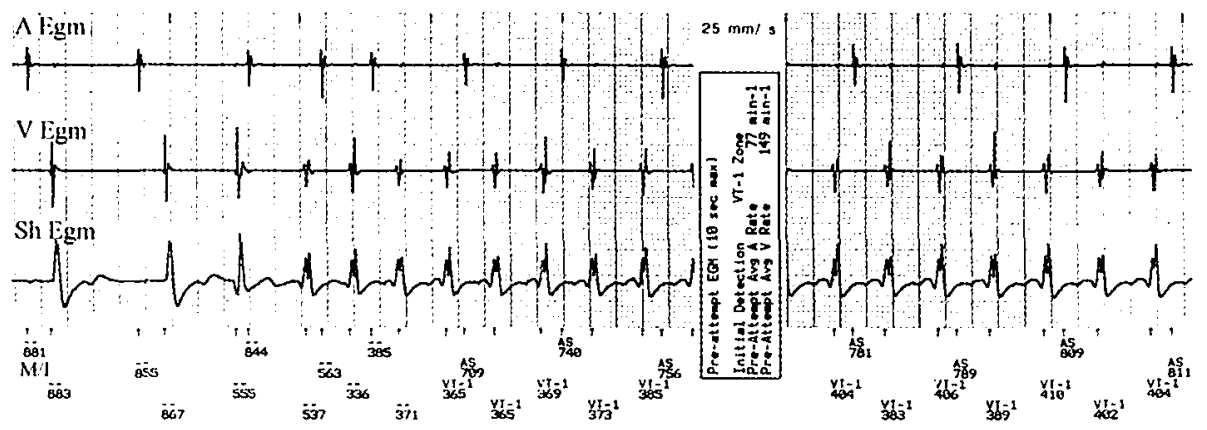

Figure 23. Dual chamber arrhythmia detection. Value of the VA conduction evaluation for the VT:SVT discrimination algorithm. ICD recordings of the A Egm, the V Egm, the marker and the interval annotation showing two episodes of spontaneous VT in the same patient. Although the cycle length is almost identical in both episodes, there is 1:1 VA conduction in the VT that starts at a higher sinus rate $(A)$ and 2:1 VA conduction in the second VT (B). Presence of retrograde VA conduction limits the use of $V>A$ algorithm as a safety measure while applying the dual chamber VT: SVT rejection rules. (Guidant Ventak 1821 AV II DR).

numerical relationship of the atrial rate to the ventricular rate; PR monotonicity- the degree of change in the timing relationship of atrial events to ventricular events. The algorithm operates in arrhythmias occurring within the two programmable VT detection zones and has its Safety Timer (32).

The Photon DR (St Jude) employs a multi-tired A-V Rate Branch algorithm that assesses the atrial and ventricular rates, dynamic rhythm characteristics and morphology criteria. The relationship between atrial and ventricular rhythm is first classified into a rate branch: $\mathrm{V}<\mathrm{A}, \mathrm{V}=\mathrm{A}, \mathrm{V}>\mathrm{A}$. Within the first two branches further evaluation of the electrogram templates, rate stability and sudden onset is applied (33). 


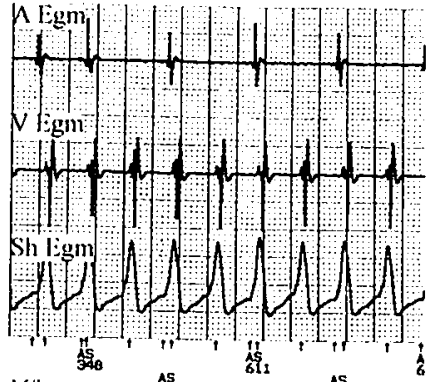

M/I

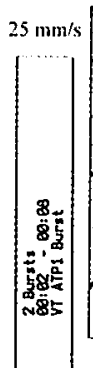

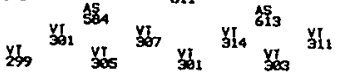

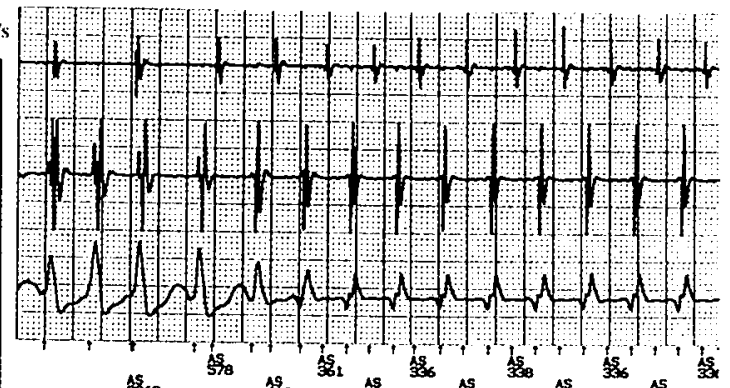

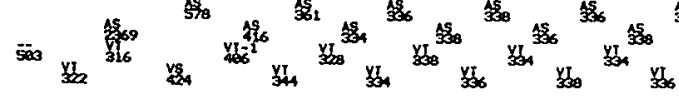

Figure 24. Dual chamber arrhythmia detection. Therapy (ATP) induced changes in VA conduction. ICD recordings of the $A$ Egm, the $V$ Egm, the Shock Egm and the marker and interval annotation during a spontaneously occurring VT episode. The VT initially has a CL of $300 \mathrm{msec}$ and no VA conduction. After two ATP sequences, the arrhythmia shows a change of morphology and lengthening of the cycle length. When the rate decreases, 1:1 VA conduction appears. Since the $V>A$ algorithm functions also on redetection, and since the retrograde conduction is critically dependent on cycle length, the value of the algorithm as the inhibitor override becomes critically dependent on the VT cycle length. (Guidant 1831 Ventak AV III DR).

\section{Ventricular arrhythmia redetection}

After delivering a therapy the ICD monitors its outcome. Generally, an arrhythmia episode is considered terminated, if during a certain non-programmable period of time none of the ventricular arrhythmia detection criteria are fulfilled. In the Medtronic ICDs this is a period of 8 consecutive VV intervals that must be longer that the VT (lowest zone) detection intervals. In the Guidant ICDs there are 10 sec (after ATP) or $30 \mathrm{sec}$ (after shock) in which none of the detection windows must be satisfied before end-of-episode is declared. A rhythm that does not satisfy the termination definition is considered part of an ongoing episode. This implicates that in case early after termination of a ventricular arrhythmia a new ventricular arrhythmia occurs, that new arrhythmia will be detected as part of the ongoing episode. As result, it misses the number of therapy tiers that were already applied for the initial arrhythmia. This rule of redetection makes electrical instability a potentially dangerous situation (fig 25). In comparison to the initial detection of a ventricular arrhythmia, the redetection criteria are easier met - the required arrhythmia duration is shorter (or a lesser number of arrhythmia intervals) and most of the arrhythmia discrimination algorithms do not function, with the rate criterion becoming again the primarily detection tool. These features of redetection are meant as a safety measure in case the first (shock) therapy 

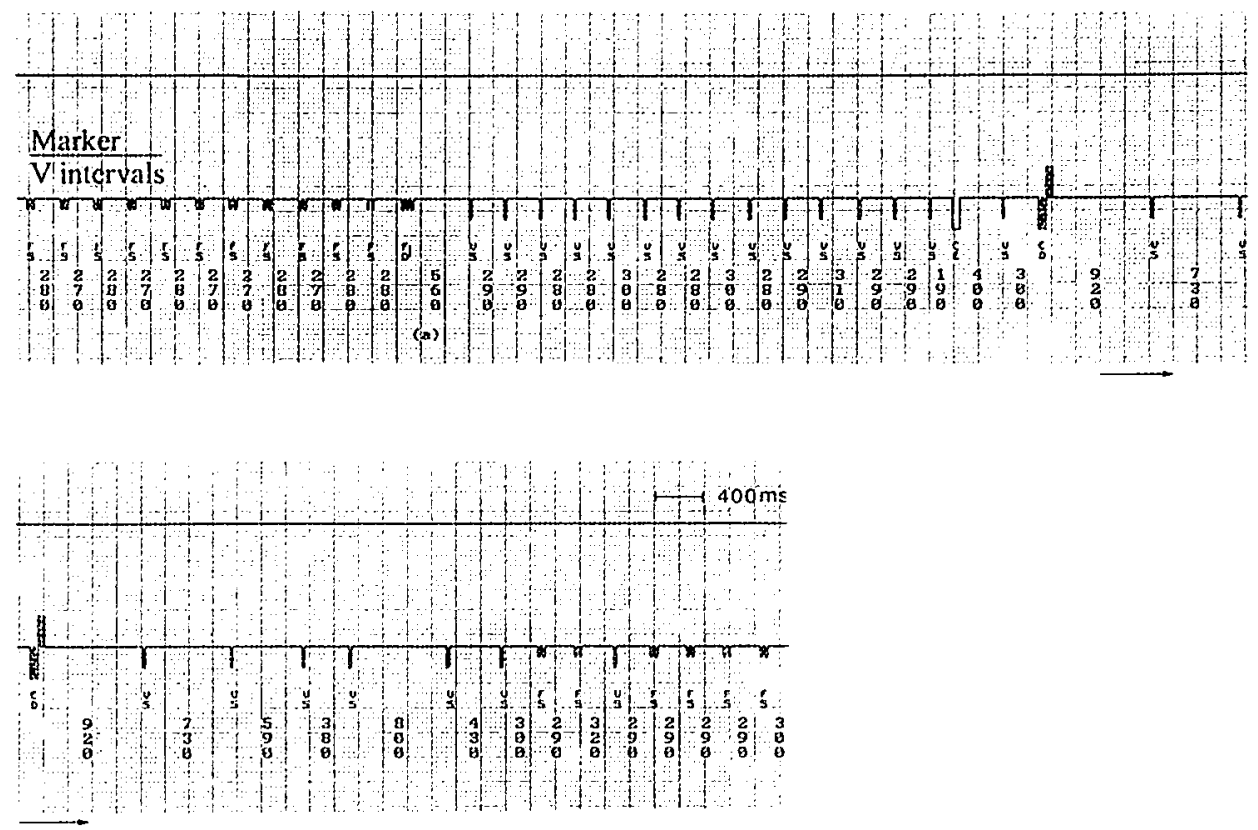

Figure 25. Ventricular arrhythmia redetection in a patient with electrical instability. The ICD recording (continuous) of one of many ventricular arrhythmia episodes occurring between two interrogation sessions. Due to limited episode storage capabilities the documentation of earlier ventricular arrhythmias derived during one interrogation, is available only in the form of the marker channel annotation and interval data. A fast VT detected in the VF zone (a) is successfully terminated by shock therapy. However within less than 8 beats a new fast VT is initiated being detected as one ongoing episode (b). The fast VT (b) will be treated with the shock therapy that was programmed as the second therapy for the fast VT (a). For each following new ventricular arrhythmia detected within the redetection time there is one therapy tier less than for the previous VT. (Medtronic 7218).

was unsuccessful, allowing a faster use of the next tiers of therapies. However this rule of increased sensitivity of arrhythmia redetection can give rise to the "second instance" inappropriate ICD therapy (fig 26).

\section{ICD ARRHYTHMIA THERAPY}

The first AIDs delivered a defibrillation shock for all types of detected ventricular arrhythmias. The AID-B model introduced in 1982 added synchronous cardioversion to the original device. It had become evident that in many of the patients, VT rather than VF was the initiating event, with the latter rhythm occurring only later, if at all. It was thought that these patients could be helped by earlier application of the shock, 

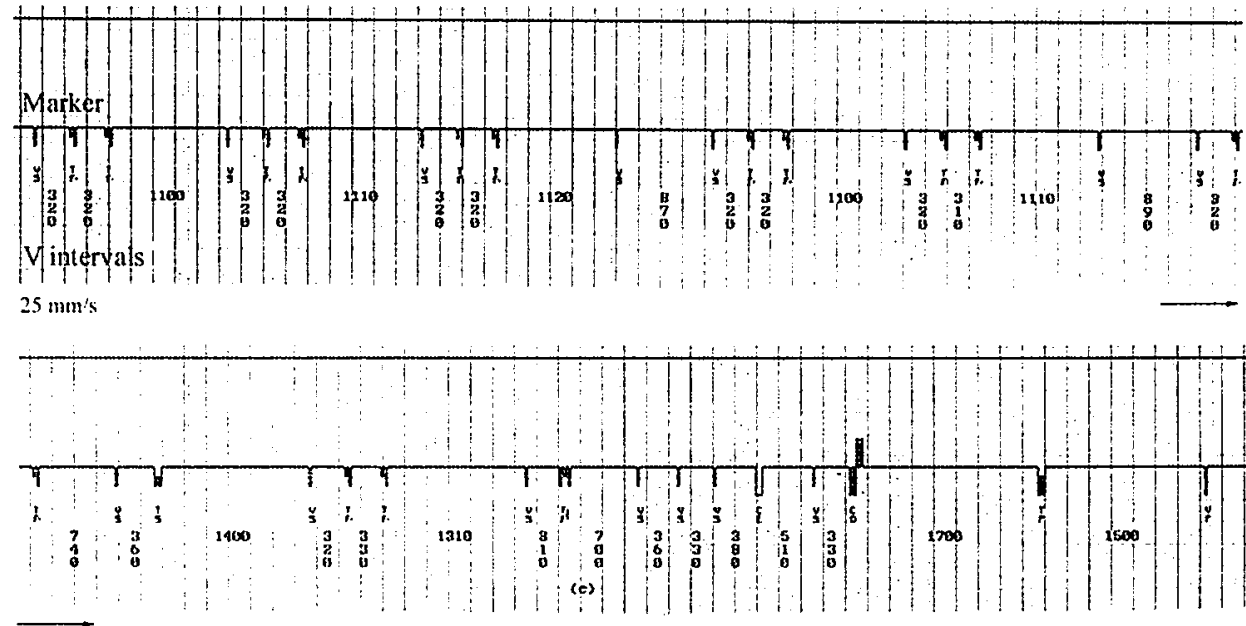

Figure 26. Ventricular arrhythmia outcome monitoring. Inappropriate redetection due to the failure to fulfill the termination criterion. Continuous recording of the marker channel and the interval annotation following the initially successfully terminated FVT. There is inability to reach the 8 consecutive non-arrhythmic beats because of the presence of many ventricular triplets. There is a short nonsustained VT present just at the moment when the capacitors are being charged and the shock is delivered. (Medtronic 7220).

thus preventing further degeneration of the rhythm. When the shock therapies became programmable in 1991 it was possible to quantify the amount of energy for different types of ventricular arrhythmia. The antitachycardia pacing (ATP) in ICDs became available in 1989 and it is now recognized that the great majority of VTs respond to this therapy. Modern ICDs have three modalities of electrical therapies.

\section{Defibrillation (DF)}

The defibrillation shock is a high voltage therapy meant as the initial treatment for VF and rapid ventricular arrhythmias, such as polymorphic VTs, that cannot be terminated with ATP. Defibrillation may be necessary as rescue therapy after a VT acceleration following ATP or low energy cardioversion.

When the defibrillation shock is to be delivered, the ICD charges the high voltage capacitors to the programmed energy. The charge time of the ICDs high voltage capacitors increases with the programmed energy, battery depletion and time since the last capacitor formation.

When the required energy level is reached, the ICD initiates a synchronization sequence during which synchronization of the DF pulse to the $\mathrm{R}$ wave is attempted. Although not required for a defibrillation shock to be delivered, the synchronization 
(attempt) is useful, because most of the ventricular tachyarrhythmias in the VF zone are very rapid VTs. In some dual chamber ICDs the synchronization sequence attempts to prevent device proarrhythmia by avoiding a shock not only during the ventricular, but also during the atrial vulnerable period.

In modern ICDs a number of physical parameters of the defibrillation pulse are programmable: the energy, the polarity, the pathway, the waveform and the tilt. Up to 6 defibrillation shocks can be delivered for one detected VF episode.

In addition, the defibrillation can be a committed or a non-committed therapy. When it is non-committed, the presence of VF must be reconfirmed by the ICD during or at the end of capacitor charging, and before the shock is delivered. This feature is useful in self-terminating ventricular arrhythmias (fig 27). The non-committed shock is available only for the first VF therapy and the next defibrillation shocks are always committed.
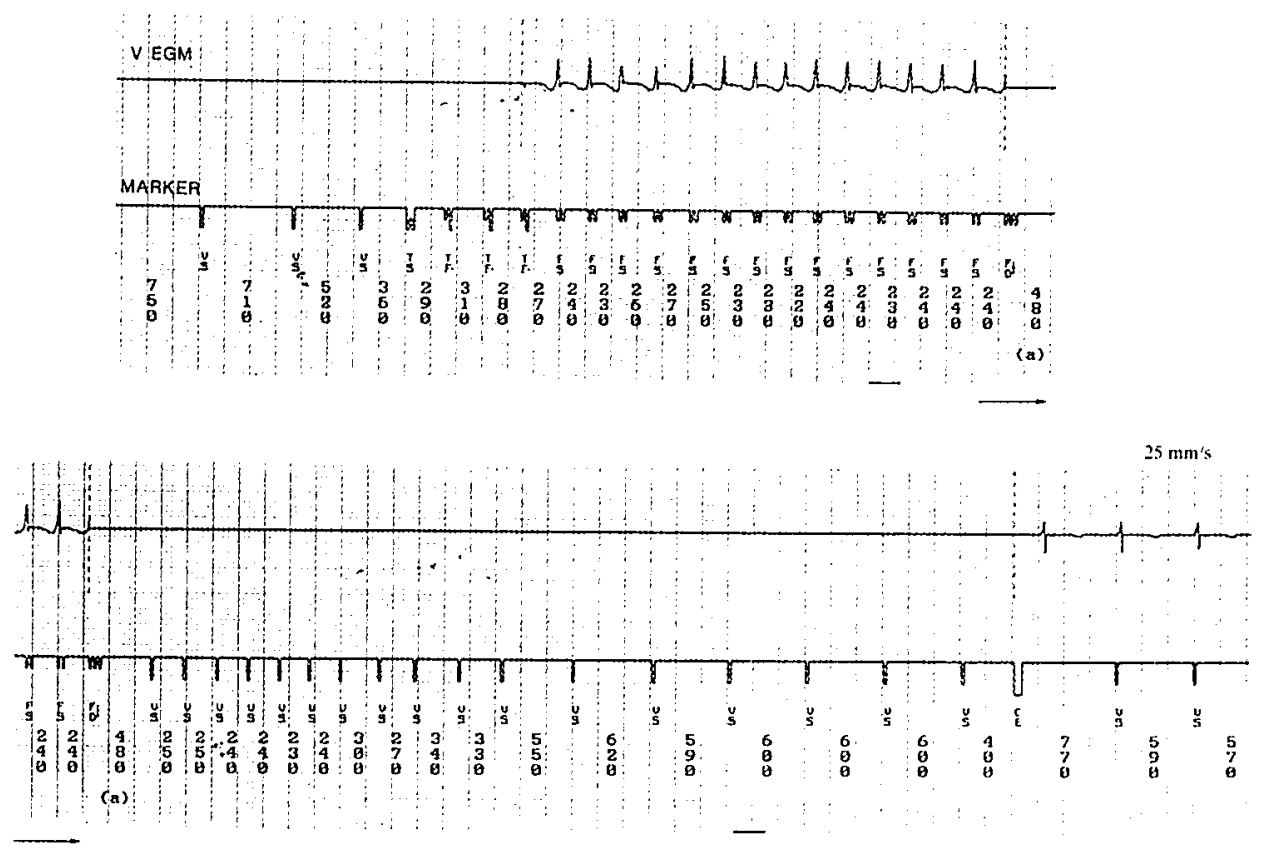

Figure 27. Non-committed defibrillation shock therapy. Value in self-terminating arrhythmias. Continuous recording of the $V$ Egm, the marker channel and the interval annotation during a spontaneous episode of a fast VT detected in the VF zone. The ventricular arrhythmia terminates spontaneously after the detection is fulfilled but before the end of the charging time. In the reconfirmation time following the end of charging only the non-arrhythmic events are present and the shock is diverted. (Medtronic 7220). 


\section{Cardioversion (CV)}

Cardioversion is a high voltage therapy meant for VTs, in which the efficacy of the pacing therapies is of limited value. This might be the case in arrhythmias having other than reentry mechanism, reentrant arrhythmia with a narrow excitable gap, after paceacceleration or in case of rapid haemodynamic deterioration during VT. The energy requirements to terminate a VT with cardioversion are less than those to terminate VF. The programmability of the shock characteristics (energy, pathway, polarity, waveform) is similar as in defibrillation therapy in the same type of ICD. In addition there is the possibility to select lower values of energy for the first and consecutive attempts.

In the Medtronic ICDs the cardioversion pulse must be synchronized to a sensed $\mathrm{R}$ wave. The requirement of confirmation makes the cardioversion a non-committed therapy.

\section{Antitachycardia pacing (ATP)}

Antitachycardia pacing involves the delivery of a series of critically timed pacing pulses in an attempt to interrupt reentrant ventricular tachyarrhythmias. In modern ICDs the basic components of the ATP are programmable such as: the type of ATP, the number of sequences delivered, the number of pulses within each sequence, the coupling interval of the pacing pulses, the shortest pacing interval. Several features of the pacing therapies are common for all types of ATP in an ICD.

The coupling interval determines the timing of the first pulse in a sequence of paced impulses. The coupling interval can have an adaptive or a fixed value. When it is adaptive, the first pacing interval is calculated based on the average of a number (for example four) of VT intervals. When it is fixed, its timing is defined in absolute time (msec), independent of the measured tachycardia interval.

The timing of pulses within a pacing sequence can also be adaptive or fixed.

The pacing pulses can never be delivered at less than the programmed minimum-pacing interval. This minimum interval limits both the VT cycle length for which the ATP is applied, but also the shortest interval within a pacing sequence. The pulse characteristics, such as, the output (amplitude and pulse width), or duration of the blanking periods are the same for all ATP therapies, but separately programmable from bradycardia pacing parameters.

\section{Types of ATP}

The ATP therapies available in modern ICDs basically stem from the Ramp or the Burst pacing schemes. In the Ramp scheme the pace-to-pace intervals are shortened within the same sequence. In the Burst scheme these intervals remain the same within the same sequence. 
The Medtronic ICDs have 3 types of ATP therapies (30).

The Ramp + scheme (fig 28A) consists of a set of VOO pulses delivered at programmable intervals. Ramp+ uses the percentage of the tachycardia cycle length to calculate both the coupling interval of the first pacing pulse and the decrements of the consecutive pulses within a sequence. If a tachycardia is redetected, the programmed Ramp+ percentages are used to calculate the pacing intervals for the new sequence. Each sequence adds one additional pacing pulse per sequence.

The Ramp scheme (fig 28B) consists of a set of VVI pulses delivered at decreasing intervals. In the Ramp sequence the first pulse is delivered at a programmed percentage of the current tachycardia cycle length and each of the remaining pulses is delivered at progressively shorter intervals by subtracting the programmed interval decrement. On redetection, the programmed percentage (of the tachycardia cycle length) and the interval decrements apply to the redetected tachycardia. Each sequence adds one additional pacing pulse per sequence.

The Burst scheme (fig 28C) consists of a set the VOO pulses delivered at equal intervals. In the Burst sequence the first pacing interval is a calculated percentage of the tachycardia cycle length and the remaining intervals remain equal. On redetection, the programmed percentage of the redetected tachycardia cycle length is applied to the first pulse, but the remaining pulses are delivered after the programmed interval decrement had been subtracted. The programmed number of pulses stays the same in the consecutive sequences.

The Guidant ICDs provide the Ramp and the Burst schemes of ATP therapies, with two more types of ATP that can be derived from the basic schemes (26).

The Ramp and the Burst are both adaptive pacing schemes showing similarity with the Ramp and the Burst in the Medtronic devices. In addition, in the Guidant ICDs the number of pacing pulses in consecutive Ramp sequences does not simply increase by one, but are programmable. The maximal number of pacing pulses is also programmable. This allows many ATP attempts without using an excessive number of pulses. The Scan scheme consists of bursts of bursts - sets of pacing trains in which the coupling interval is adaptive only in the initial burst, while thereafter the cycle length of successive bursts is systematically shortened by a programmed scan decrement. Except for the first sequence, the ATP is of the fixed type.

The Ramp/Scan scheme consists of bursts of ramps - sets of pacing trains in which the coupling interval is adaptive only in the initial Ramp, while thereafter the cycle length shortens within a Ramp (ramp decrement) and among successive sequences (scan decrement). Except for the first sequence, the ATP is of the fixed type. 
A Ramp +

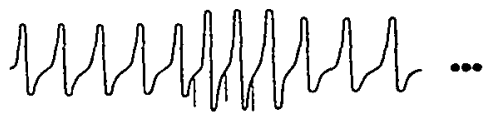

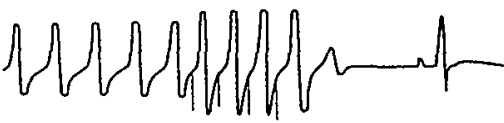
$350 \mathrm{~ms}$
WV Average

260240230

Ramp+ Therapy

$350 \mathrm{~ms}$ WV Average

260240230230 Sequence

Ramp+ Therapy

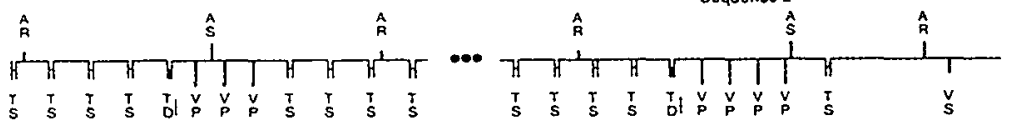

$\begin{array}{lll}\text { Programmed Values: } & \text { Initial Number of Pulses }=3 & \text { R.S1 }=75 \% \\ & \text { Number of Sequences }=4 & \text { S1.S2 }=69 \% \\ & \text { Minimum interval }=230 \mathrm{~ms} & \mathrm{~S} 2 \cdot \mathrm{SN}=66 \%\end{array}$

B

Ramp
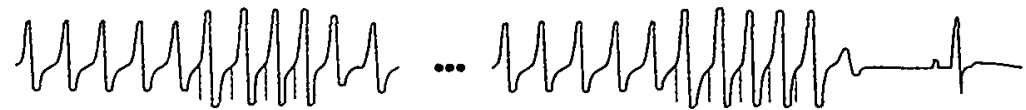

$350 \mathrm{~ms}$
W Average

320310300290

$350 \mathrm{~ms}$
W Average

$\underbrace{320310300290280}_{\text {Ramp Therapy }}$

Sequence 2
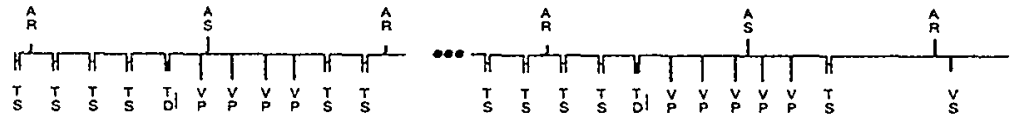

Programmed Values:

Initial Number of Pulses $=4$

Decrement per interval $=10 \mathrm{~ms}$

Number of Sequences $=4$

Minimum intenal $=250 \mathrm{~ms}$

R-S1 $=91 \%$

C

Burst
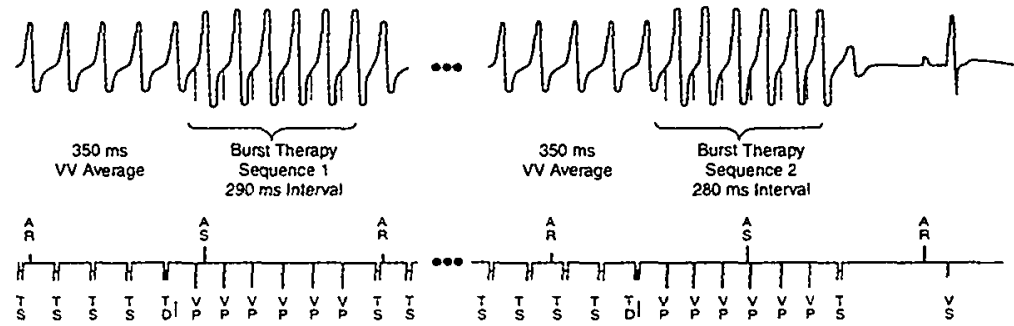

$\begin{array}{lll}\text { Programmed Values: } & \begin{array}{l}\text { Number of Pulses }=6 \\ \text { Number of Sequences }=4\end{array} & \begin{array}{l}\text { Decrement per Sequence }=10 \mathrm{~ms} \\ \text { Minimum Interval }=230 \mathrm{~ms}\end{array} \\ \text { R.S1 }=84 \% & \end{array}$

Figure 28. Ventricular adaptive antitachycardia pacing therapies. Schemes of the Ramp+(A), the Ramp (B), the Burst (C). See test for explanation. (30, with permission of Medtronic). 


\section{Atrial therapies}

The Medtronic 7250 ICD model is the first dual chamber device in which different types of electrical therapies for sustained atrial arrhythmia are available (30). Adaptive antitachycardia pacing (ATP) is available for AT, a $50 \mathrm{~Hz}$ burst and atrial cardioversion for both AT and AF. Atrial cardioversion can be automatic or commanded by a patient. ATP and the $50 \mathrm{~Hz}$ burst function as automatic therapies.

Adaptive antitachycardia pacing therapies can be of two modalities, the Ramp or the Burst + . In both types of atrial ATP, the coupling interval of the first pulse, the initial number of pulses, the interval decrements, the number of sequences and the minimum AA interval are programmable. Both Ramp and Burst+ in the atrium function differently as compared to those in the ventricles.

The Burst + scheme (fig 29A) consists of sequences of an AOO burst, in which the coupling interval and the first two stimuli are adaptive as a percentage of the detected atrial arrhythmia cycle length. On redetection the coupling interval remains adaptive, but the timing of the remaining pulses within a burst are calculated by subtracting the programmable decrement interval.

The Ramp scheme (fig 29B) consists of sequences of an AOO burst delivered at decreasing intervals. The first pulse in the sequence is adaptive to the detected atrial arrhythmia cycle length. The remaining intervals within a sequence are delivered at progressively shorter (programmable) intervals. On redetection the coupling interval is also adaptive and the interval decrement has the same programmed value with an extra pulse added to each sequence.

$50 \mathrm{~Hz}$ burst therapy consists of bursts of $20 \mathrm{msec}$ interval AOO pulses delivered during a programmed time (in seconds).

Atrial cardioversion has programmable features such as high voltage pulse characteristics (energy, pathway, polarity) and therapy availability (synchronization, sustained duration timer, patient activated vs automatic initiation) and a time window during the day for cardioversion to be delivered for sustained AF.

\section{BRADYCARDIA PACING}

In the first generation ICDs antibradycardia pacing was not present. With post-shock and anti-bradycardia stimulation the pacemaker became an integral part of the ICD systems in 1988. The ability to adjust the sensing level and the duration of the blanking and refractory periods during paced and sensed rhythm was a challenge. During detected ventricular arrhythmias bradycardia pacing is suspended. The concern for possible undersensing of VF was the reason for always committed behavior of the shock therapy during bradycardia pacing in older types if ICD (fig 30). For several 
A A Burst +

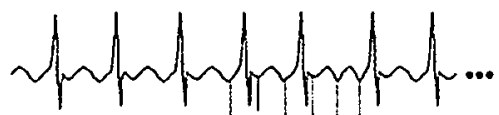

$280 \mathrm{~ms}$ AA Avorago $-240 \mathrm{~ms}-{ }^{210}{ }_{100}$

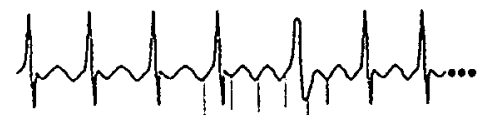

A.Bursl+ Sequence

$280 \mathrm{~ms}$
AA Avolago

A.Burst + Sequence 2

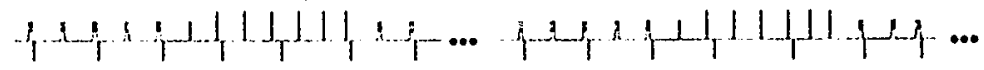

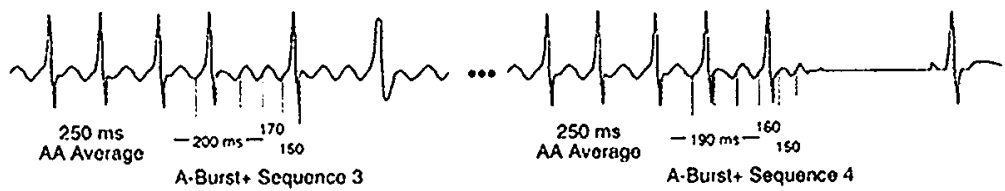

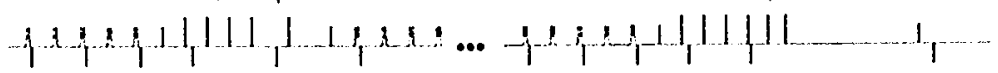

$$
\begin{aligned}
& \text { Programmod Values: Initial Number of Pulses }=4 \quad \text { A.S1 }=87 \% \\
& \text { Numbor of Sequencos = } 4 \quad \text { S1.S2 }=75 \% \\
& \text { Minimum Intorval }=150 \mathrm{~ms} \quad \mathrm{~S} 2 . \mathrm{S3}=69 \% \\
& \text { Decrement por Soquenco }=10 \mathrm{~ms}
\end{aligned}
$$

B

A Ramp
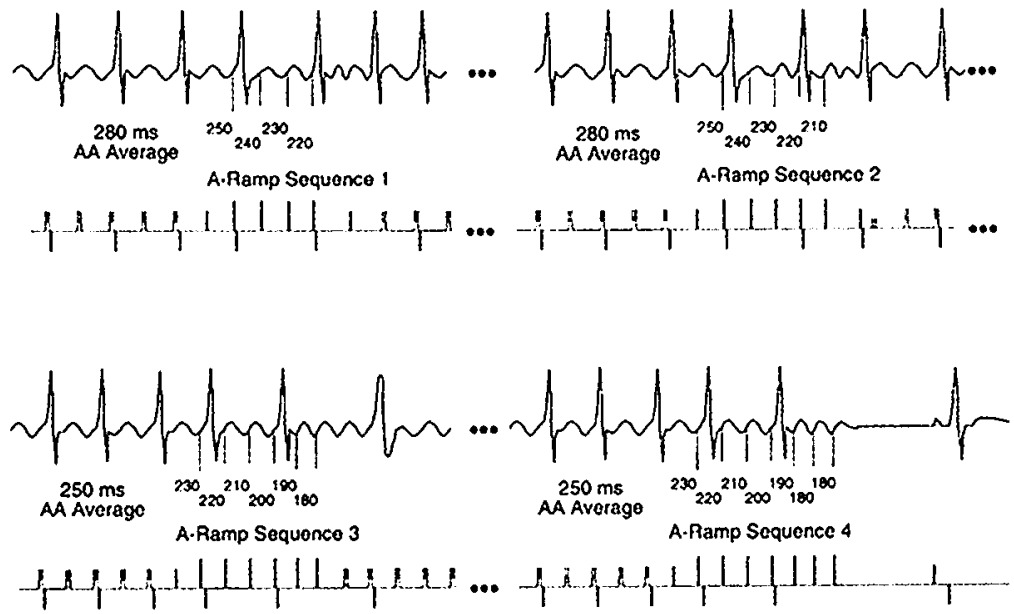

$$
\begin{array}{ll}
\text { Programmed Values: } & \begin{array}{l}
\text { Initial Number of Pulses }=4 \\
\text { Number of Sequences }=4 \\
\text { A.S } 1=91 \%
\end{array}
\end{array} \quad \begin{aligned}
& \text { Decremenl per Interval }=10 \mathrm{~ms} \\
& \text { Mituimum Interval }=180 \mathrm{~ms}
\end{aligned}
$$

Figure 29. Atrial adaptive antitachycardia pacing therapies. Schemes of the Burst+ (A) and the Ramp (B). See text for explanation. (30, with permission of Medtronic). 


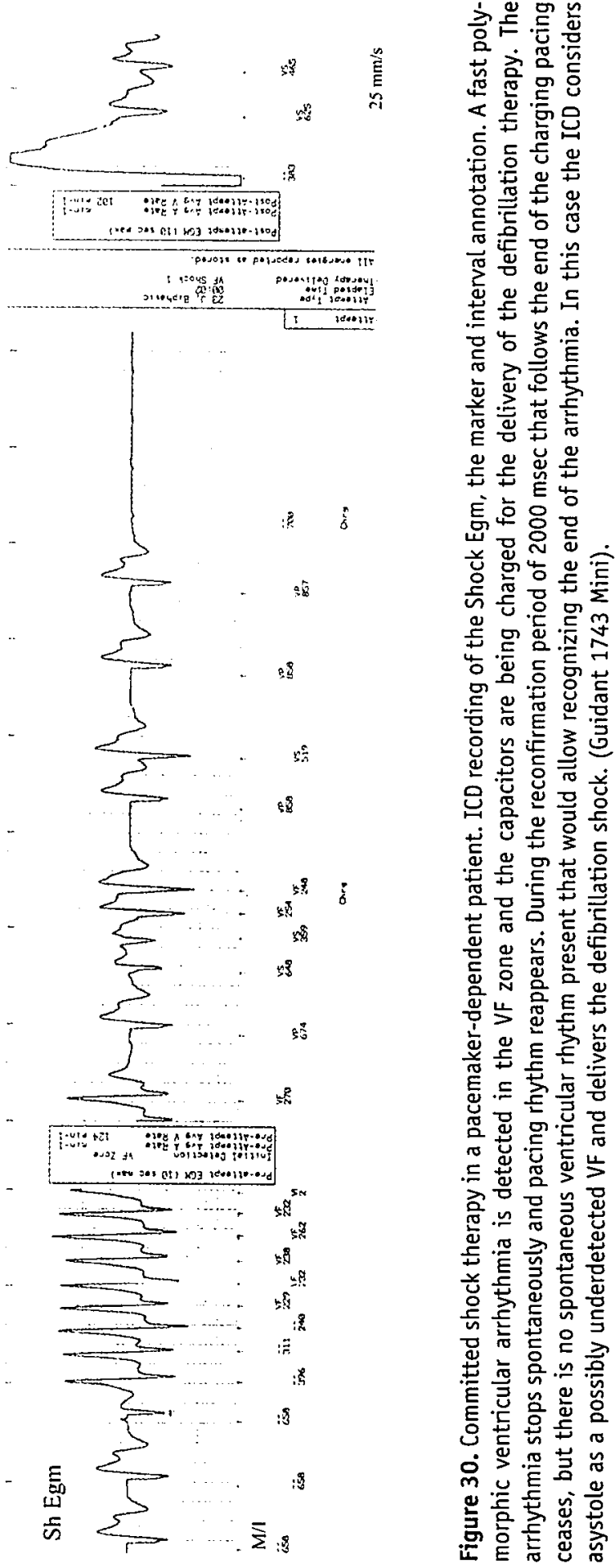


years the "bradycardia part" of the ICD, being reduced to a simple VVI mode, stayed behind the standards available in separate pacemakers (fig 31). In selected ICD recipients who were dependent on (more sophisticated) cardiac stimulation, two separate systems were implanted in some centers. Dual chamber ICD technology in 1995 led to a breakthrough in bradycardia therapy in ICD patients. The value of the dual chamber devices turned out to be more than only the ability of AV sequential stimulation (fig 32). Dual chamber technology created the possibility for new features that were very relevant in patients with diseased hearts and an ICD. The physiological sensors available since 1997 improved rate responsiveness in patients with chronotropic incompetence due to antiarrhythmics or underlying heart disease. At the same time mode-switching algorithms prevented fast ventricular tracking of paroxysmal atrial arrhythmias. Dual chamber pacing algorithms for atrial and ventricular rate stabilization can be of value for preventing pause-dependent arrhythmia initiation and can function as rate smoothing in patients symptomatic with premature beats (fig 33). With the development of biventricular stimulation in 1999 ICD-integrated pacemaker technology has achieved, what was until then available only for the pacemaker patient.
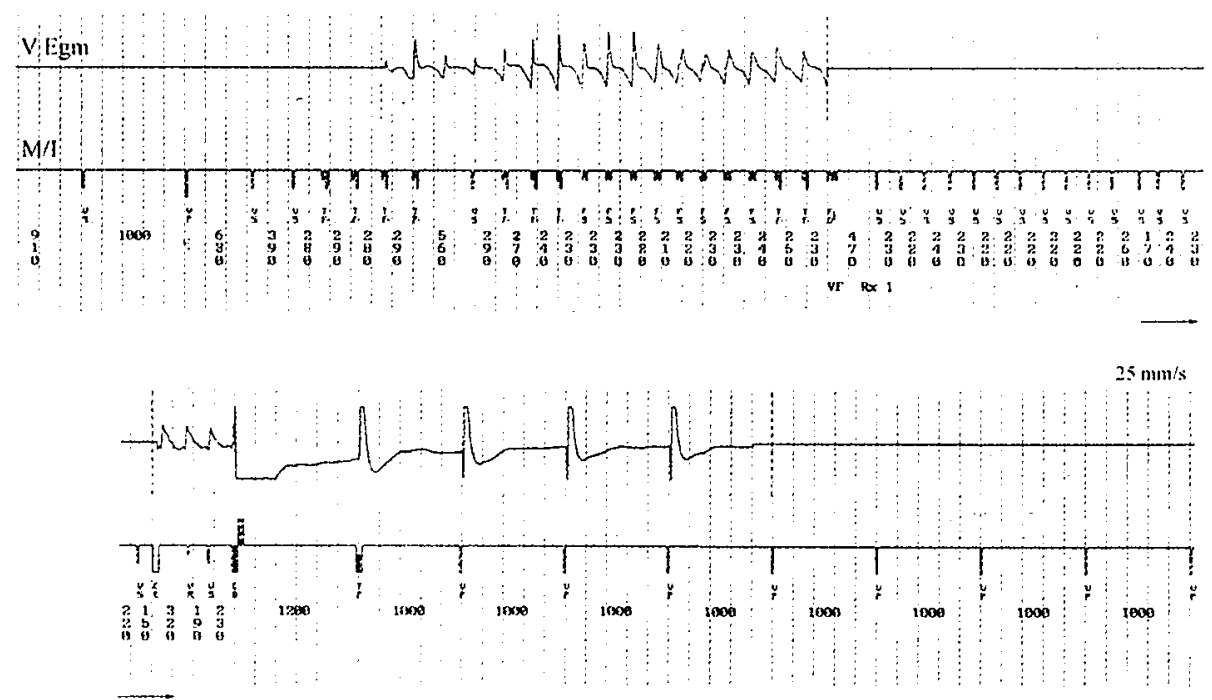

Figure 31. Post shock bradycardia pacing in a single chamber ICD. Continuous recording of the $V$ $\mathrm{Egm}$, the marker channel and the interval annotation during an episode of a spontaneous ventricular tachyarrhythmia. The fast pleomorphic VT was detected as VF and treated with a defibrillation shock. The patient consciously experienced the shock but became near syncopal thereafter. Pacing in the VVI mode after the episode of ventricular arrhythmia is not sufficient to allow fast restoration of haemodynamics. (Medtronic 7219). 


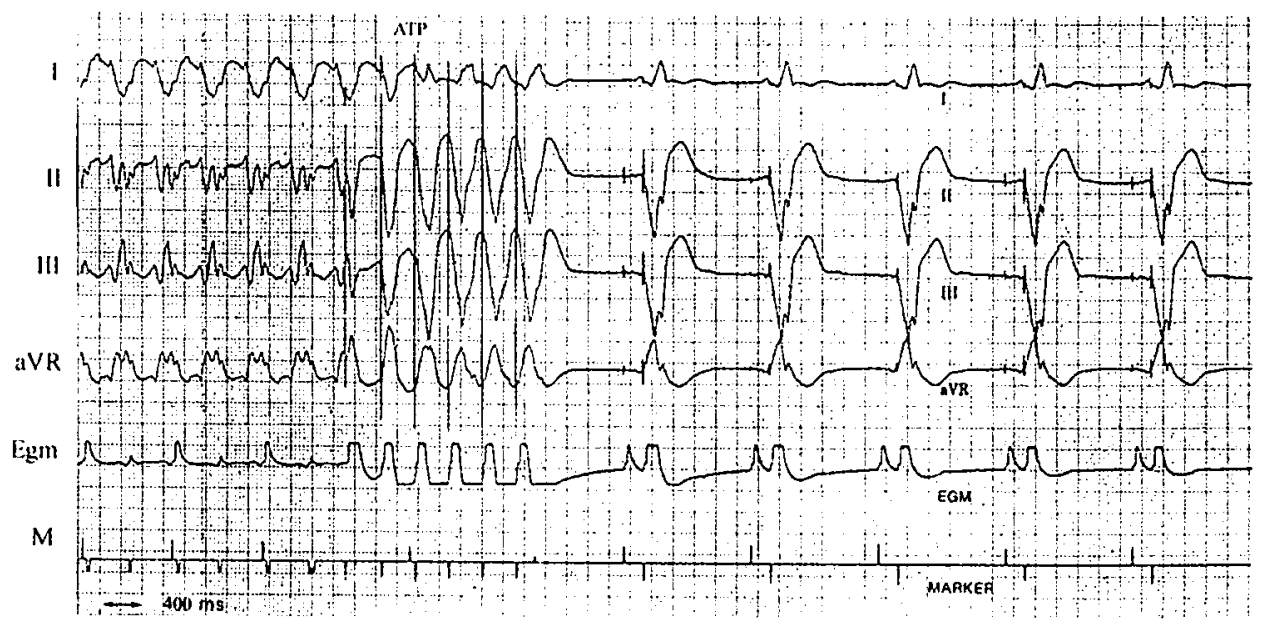

Figure 32. Dual chamber pacing for bradycardia following ATP termination of a sustained monomorphic VT. Shown are the extremity leads ECG, the wideband Egm and the marker channel registration of a VT induced during predischarge testing. The marker channel discloses the sinus rate being 70 beats/min during the VT. Following pace-termination of VT the sinus rate becomes slower than the programmed DDD escape interval, and the spontaneous AV conduction time becomes longer than the programmed AV interval of $200 \mathrm{msec}$. As shown, neither a shock therapy, nor 1:1 VA conduction during VT are necessary to suppress normal rhythm formation and conduction.

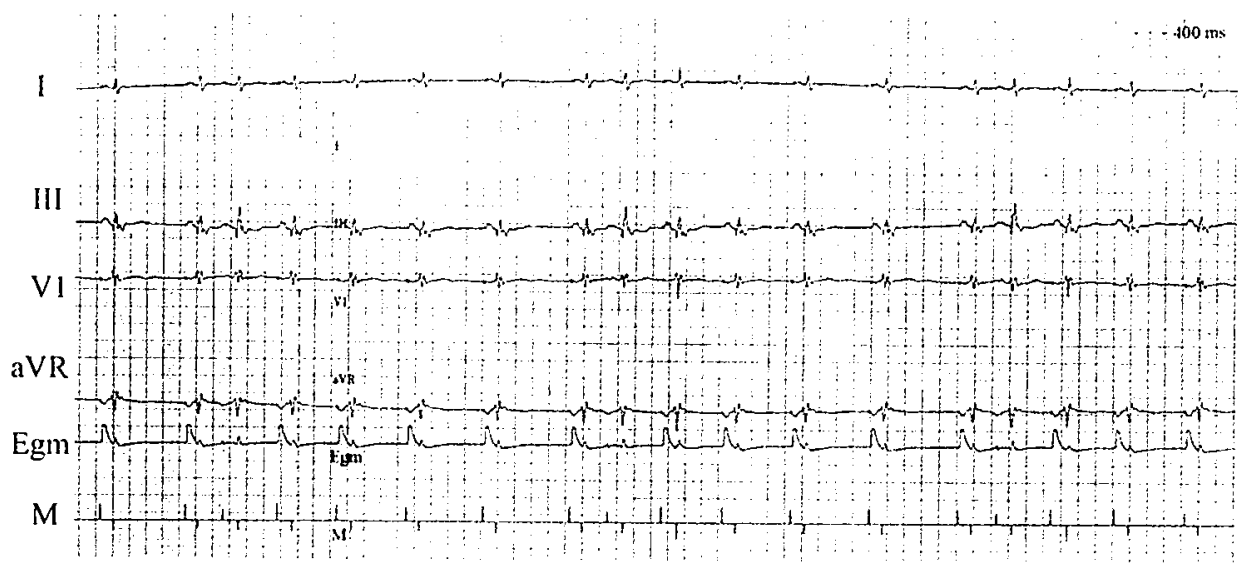

Figure 33. Dual chamber pacing for arrhythmia prevention. Atrial Rate Stabilization. Tracing showing four surface ECG leads, the wideband Egm and the marker channel, registered during spontaneous rhythm outside the arrhythmic episode. The Atrial Rate Stabilization (ARS) algorithm is meant to prevent the onset of these atrial arrhythmias that depend on a short-long-short sequence following atrial premature beats. Each atrial premature beat causes shortening of the atrial pacing escape interval, which is than gradually lengthened until the intrinsic rate. The marker registration confirms that only APBs (some with aberration on the surface ECG) trigger ARS pacing. In the presence of frequent $A P B s$ the mean heart rate can be higher than the programmed lower rate. (Medtronic 7250 Jewel AF). 
Importantly, even in modern devices the ICD and the pacemaker cannot function independently from each other due to safety requirements for both parts of the system. The requirement that the detection of the ventricular arrhythmia should never be jeopardized by heart stimulation has consequences for the availability of pacing rates and duration of blanking and refractory periods in a pacemaker within the ICD system. The pacing blanking and refractory periods are intended to prevent sensing of pacing related evoked responses and/or $T$ waves by these sensitive devices. These oversensing phenomena may inappropriately be detected as arrhythmic events (fig 34). On the other hand blanking and refractory periods that are to long in relation to the heart rate, might lead to narrowing of the detection window for ventricular arrhythmias. Therefore the upper rate behavior in the ICD - integrated pacemakers is additionally guarded by parameter interlocks to protect safe ventricular arrhythmia detection (fig 35). Despite these initial limitations, dual chamber technology underwent an accelerated evolution and has become a valuable part of ICD therapy.
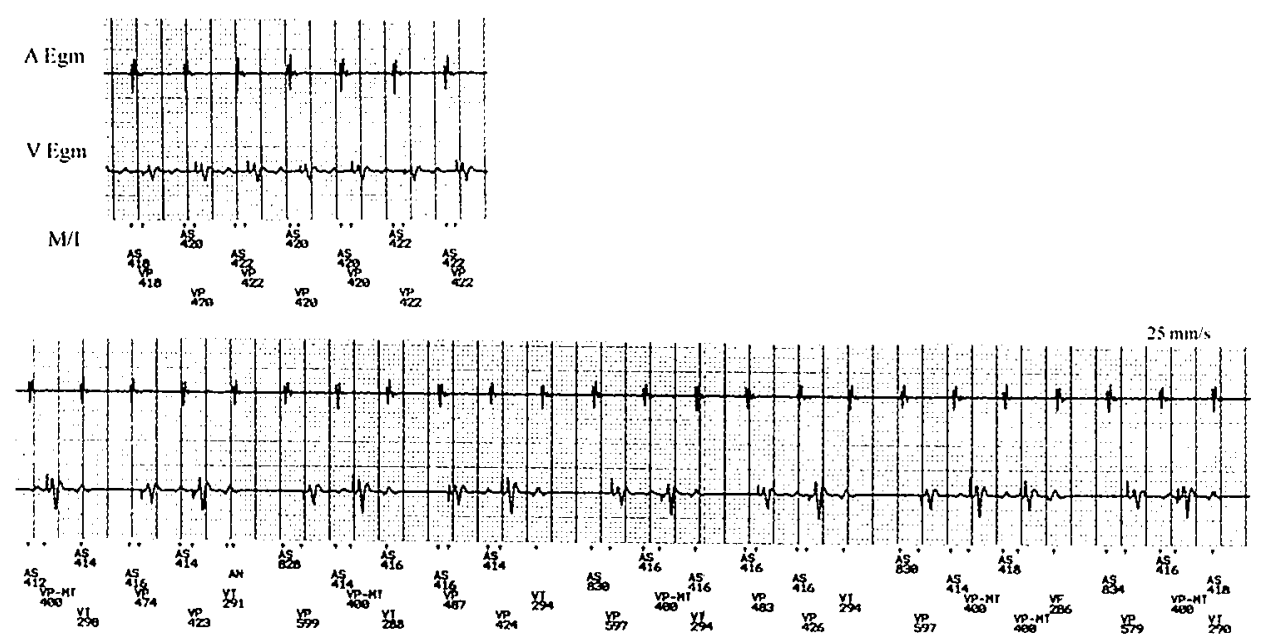

Figure 34. Sensing interactions in the ICD integrated DDD pacemaker caused by the ICD protecting the ventricular arrhythmia detection. Tracings showing the A Egm, the V Egm, the marker and interval annotation registered during an exercise test. The maximum tracking rate was programmed to 150 beats/min, the duration of the non-programmable blanking period for ventricular paced events is $250 \mathrm{msec}$, the dynamic ventricular refractory period shortens at higher pacing rates to allow detecting the initiation of ventricular arrhythmias, but cannot be less than the ventricular blanking period. A) When the heart rate reaches the maximum tracking rate $T$ - wave oversensing occurs, classified by the device as VT or VF sensed event. The arrhythmic sensed events inhibit ventricular pacing, reducing the effective bradycardia-pacing rate in a 3:2 and 4:3 manner. An important contributor to T wave oversensing is the automatic gain control, which is set to the maximum value after ventricular paced beats. B) The adjustment of the sensitivity to "less sensitive" settings was in this case sufficient to solve the problem. (Guidant 1821 Ventak AV II DR). 

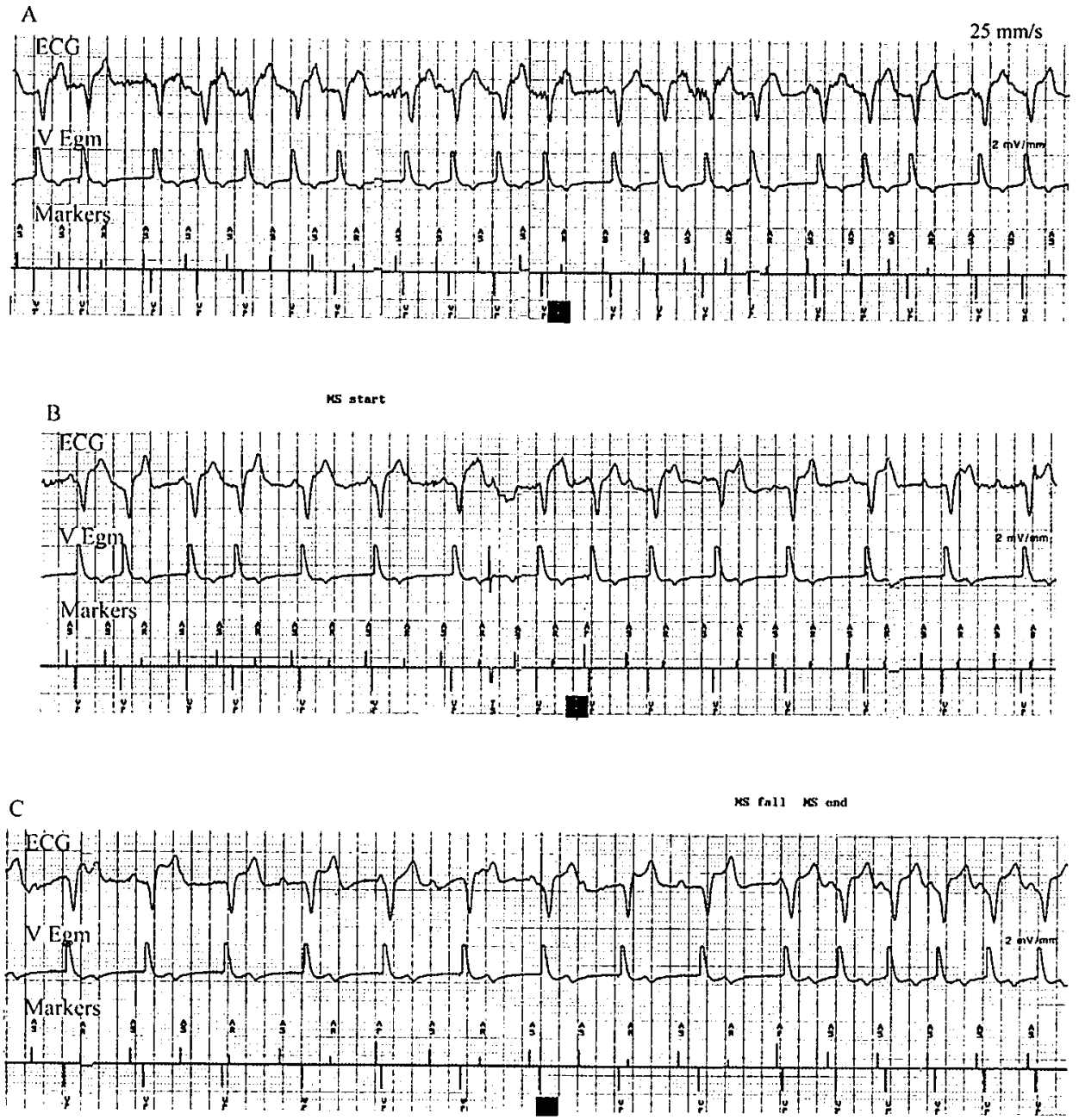

Figure 35. Upper rate behavior of the DDD pacemaker integrated in the first generation of the dual chamber arrhythmia management device. Tracings showing the single lead ECG, the V Egm, and the marker channel annotation registered during an exercise test. The maximum programmable upper rate in the device is limited to 120 beats/min and the shortest duration of the blanking period of ventricular paced events is $200 \mathrm{msec}$. If the cycle length of the ventricular paced rhythm is $500 \mathrm{msec}$ the window to detect the initiation of the ventricular arrhythmia is $300 \mathrm{msec}$. In an attempt to protect the detection of the initiation of ventricular arrhythmias, the device limits the time during which that high paced ventricular rate narrows the detection window. Sinus tachycardia of more than 120 beats/min causes the occurrence of the pacemaker Wenckebach periodicity (A). When the duration of Wenckebach pacing fulfills the AF evidence counter, mode switching occurs (B). When the sinus rate decelerates below the upper rate limit there is $A V$ resynchronization (C). (Medtronic 7250 Jewel AF). 


\section{ELECTROPHYSIOLOGICAL STUDY}

The lack of the possibility of cardiac pacing in the first generation ICD meant inability of non-invasive evaluation of arrhythmia induction and termination. Ventricular arrhythmia induction, necessary to test the detection and electrical therapies of the device required invasive methods using external stimulators and fibrillators. Determining and verifying the energy requirement for VF termination was usually the only test done. In 1988 while "slaved" VF induction was still applied during the implant procedure, VF induction using AC and VT induction using programmed electrical stimulation (PES) in the ventricles could be performed via the device during predischarge testing. The introduction in 1993 of the $T$-wave shock as a manner of VF induction (fig 36), made the AC bursts unnecessary in almost all patients (fig 37). In addition, in some patients VF is induced during the programmed electrical stimulation meant to induce VT (fig 38). The availability of programmed electrical stimulation within an ICD allowed VT induction and evaluation of optimal therapies. From1995 the device-based ventricular arrhythmia testing during the implant procedure has become standard.

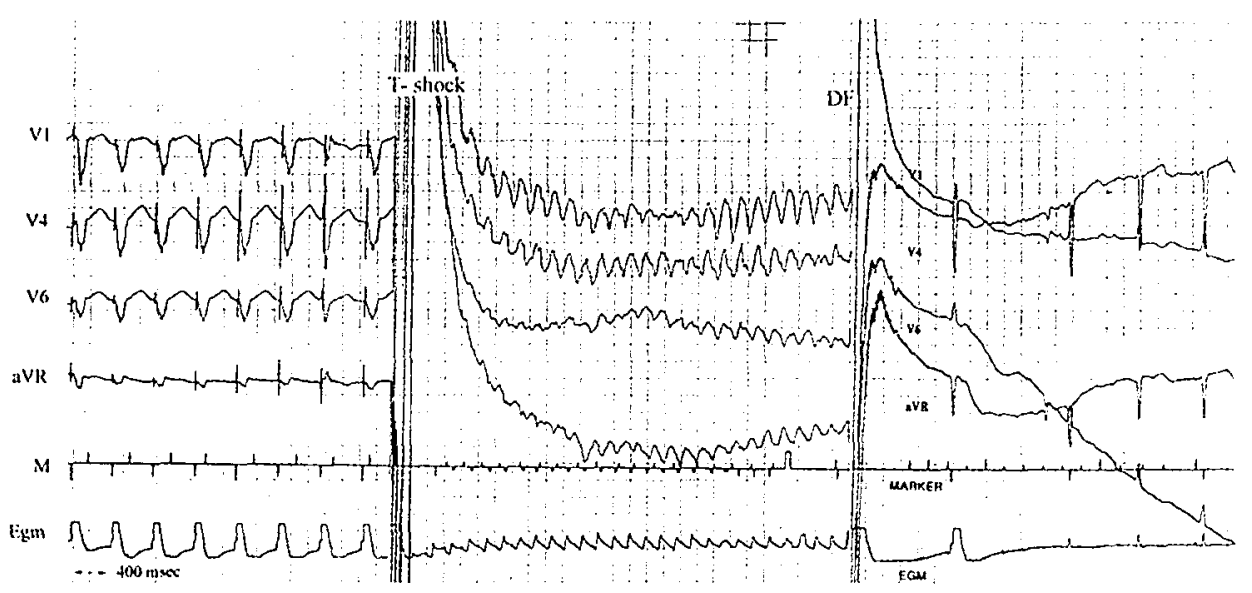

Figure 36. Predischarge testing. VF induction using $T$ wave shock. Shown are tracings of four surface $E C G$ leads, the marker channel and the wideband $A+V$ Egm registration. Following the train of 8 beats a low energy (usually 1 Joule) shock is applied in the T wave, resulting in VF (and AF) induction. The $V F$ is adequately sensed and detected, the dual chamber discrimination algorithm is not allowed in the VF zone. Following defibrillation therapy the VF terminates, the (possibly reinitiated) AF continues during 4 more RR intervals. The VF induction and termination sequences are used to determine the lowest successful energy to terminate the VF. 


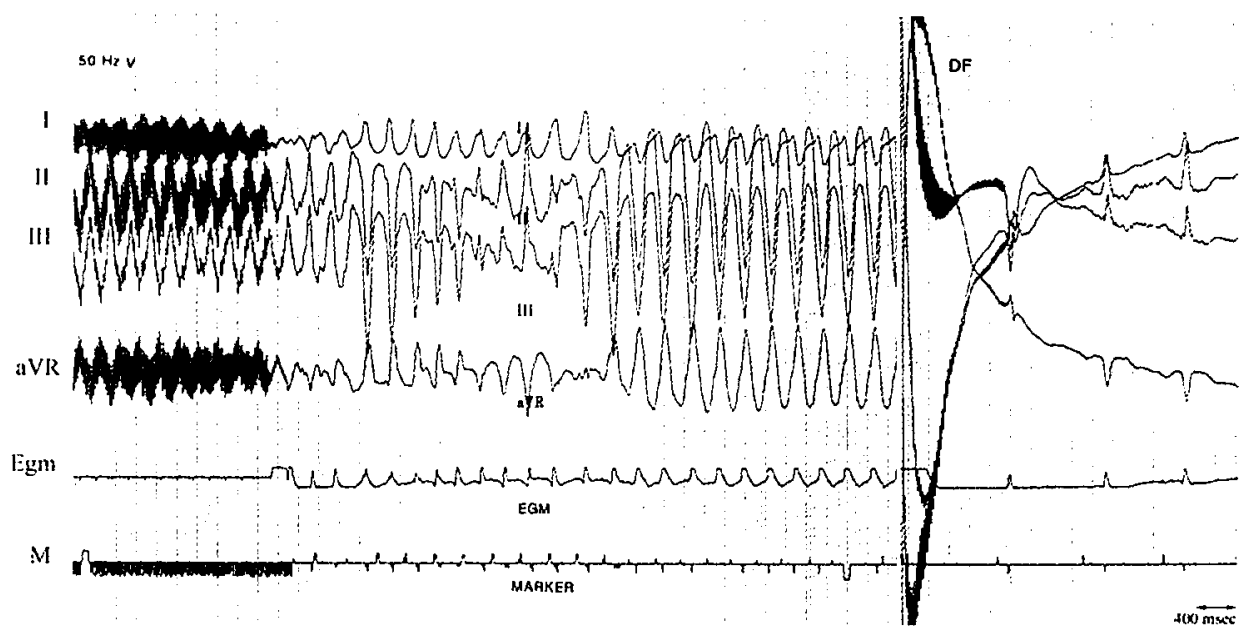

Figure 37. Predischarge testing. VF induction using a $50 \mathrm{~Hz}(20 \mathrm{msec})$ ventricular burst. Shown are tracings of four surface ECG leads, the wideband Egm and the marker channel registration. Applying a $50 \mathrm{~Hz}$ burst during several seconds is a mode of VF induction, which was used in older generations ICDs to test defibrillation efficacy. Being considered more arrhythmogenic than the T-wave shock, it is at the present time used only when the last one fails to induce VF. But even with the $50 \mathrm{~Hz}$ burst it is in some patients difficult to obtain a sustained VF as illustrated in this figure. The ECG during burst application suggests the presence of ventricular flutter and the induced arrhythmia starts as a fast polymorphic VT. However, the polymorphic VT organizes into a fast monomorphic VT. As a consequence, the arrhythmia that is terminated by the defibrillation shock is not true VF. Patients in whom an initially induced VF organizes into VT are mostly using antiarrhythmic drugs and can present VF in the clinical setting. Therefore they require to be tested for termination of true VF.

The purpose of predischarge testing has been changing parallel to the development of new ICD technologies. At the time when the hardware of ICD systems was undergoing their most dramatic evolution, certainty about the safety of these changes was of utmost importance. Steps from epicardial to nonthoracotomy lead system, from abdominal to pectoral implant, from the monophasic to biphasic shock waveform, from the "cold can" to the "active can", from double to a single defibrillation lead from a large to a small can, had first to be validated carefully. At the same time for many years the primary indication for the ICD was cardiac arrest due to VF. Those were the reasons why in the first generations of ICD VF induction and termination received most attention while testing the ICD. The VF defibrillation threshold (DFT) was determined during the implant procedure, before hospital discharge and its stability was often confirmed at three months follow-up. Since 1995 the changes in ICD hardware for VF therapy have been less dramatic than before and were directed towards further reduction of the ICD volume. At the same time the indications for ICD implant were widened and patients with symptomatic and even asymptomatic VTs 


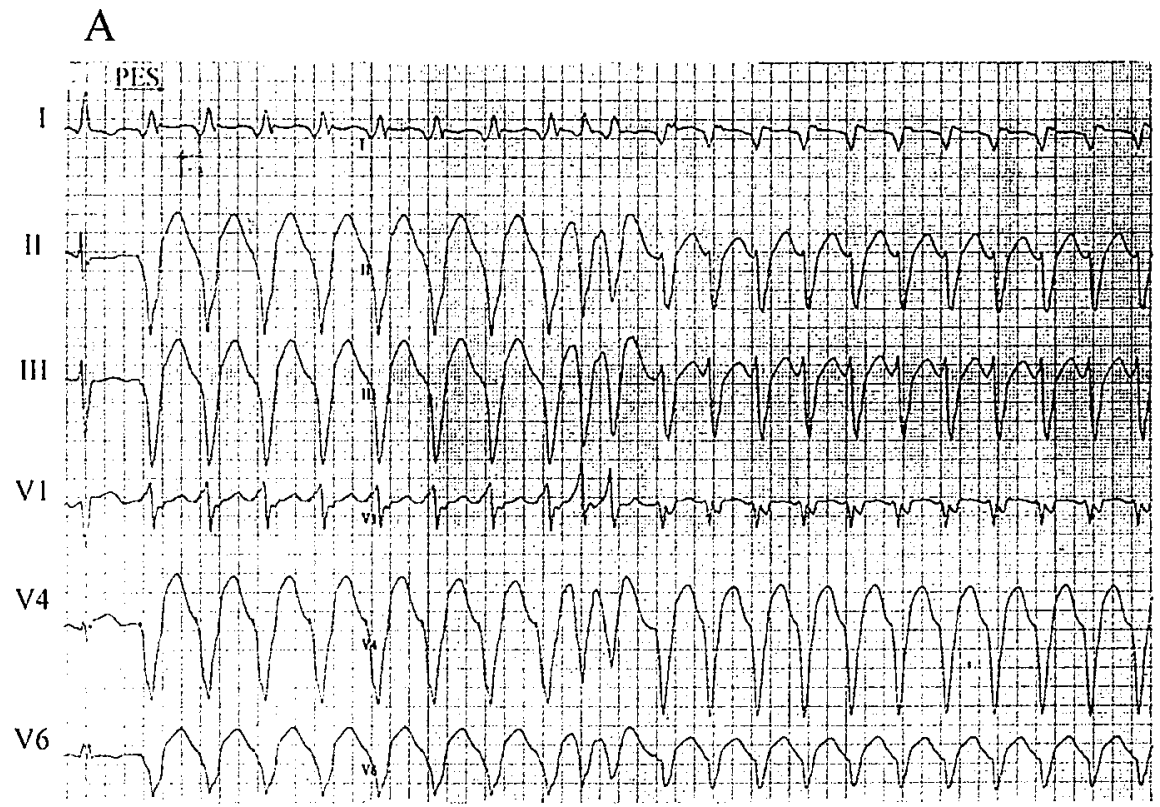

B

1 a

11

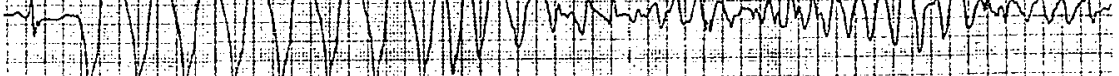

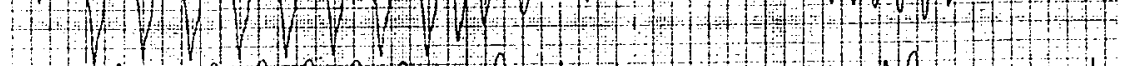

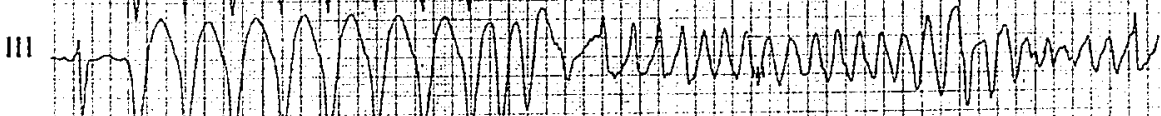
10 Nof

VI

V4

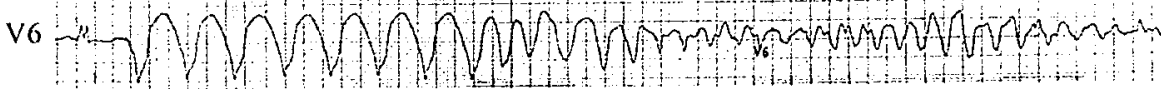

$\rightarrow 400 \mathrm{msec}$

Figure 38. Predischarge testing. Programmed electrical stimulation resulting in ventricular arrhythmias of different severity in the same patient. Shown are six surface ECG leads. Programmed electrical stimulation was performed to study the clinical VT, which was induced using a train of 8 stimuli of $600 \mathrm{msec}$ followed by two ventricular premature beats (A). As shown in panel $B$ a basic train of $500 \mathrm{msec}$ and two ventricular premature beats results directly in VF induction. 
became a growing group among the ICD recipients. Therefore, while the determination of the DFT remains the most important test during the implant procedure, predischarge testing has become a useful opportunity to test different types of ICD therapy, as well as the more and more complex detection algorithms.

In the VT patient antitachycardia pacing, if feasible, is by far the best way of arrhythmia termination. Low energy cardioversion is the second modality of VT termination and the energy requirements for VT termination are almost always less then for VF. It is clinically relevant to evaluate the arrhythmia - ICD interactions, while applying these therapies. To begin with, it is very valuable to evaluate the clinical ventricular arrhythmia, its type, rate, morphology and haemodynamic tolerance. For haemodynamic stable VTs more ATP attempts are allowed. In patients with multiple morphologies of VT, the arrhythmias might respond differently to ATP requiring differentiation of the ATP parameters, such as the coupling interval, the number of pulses or the number of sequences (fig 39). In some patients successful pace- termination of their VTs is critically dependent on the ATP parameters and might be poorly reproducible (fig 40). In contrast, in other patients the VTs show a wide excitable gap or tend to terminate spontaneously (fig 41). The role of antiarrhythmic drugs prescribed with the intention to slow the VT making it accessible for pacing therapies need to be evaluated in the individual patient. The effect of antiarrhythmic medication on conduction times and refractory periods, in relation to different heart rates, autonomic tone, and other factors (like presence of myocardial ischemia or physical exercise), may be complex and not necessarily beneficial. On the other hand also a fast VT can be successfully terminated with ATP in patients having a beta-blocker as their only drug (fig 42). Patients with frequent nonsustained VTs, or nonsustained polymorphic VTs may benefit from longer detection times, which allow spontaneous termination of their arrhythmias. In contrast, shorter detection times might be better in fast VTs showing a spontaneous tendency to degenerate into VF (fig 43).

The outcome of the ICD treatment is determined not only by the efficacy of the electrical therapies but also by the potential device induced proarrhythmia, for which susceptibility might differ among individual patients. Pace-acceleration of VT may be prevented by adjustments of ATP parameters (number, coupling intervals), making them less aggressive $(34,35)$. When the programmed number of ATP attempts is not successful the therapy progresses to low energy cardioversion. Although cardioversion is accompanied by patient discomfort, it is usually preferable to pace-acceleration into VF. High-energy therapy needs evaluation of its proarrhythmic potential as well (fig 44). Shock-acceleration of VT may be prevented by adjustments of parameters, such as strength, waveform; reprogramming of detection zones, to ensure $R$ wave synchronized cardioversions. Device proarrhythmia can also occur at the supraventricular level, resulting in "second instance" inappropriate therapy. Considering that most arrhythmia discrimination algorithms do not operate on redetection in patients with 
A

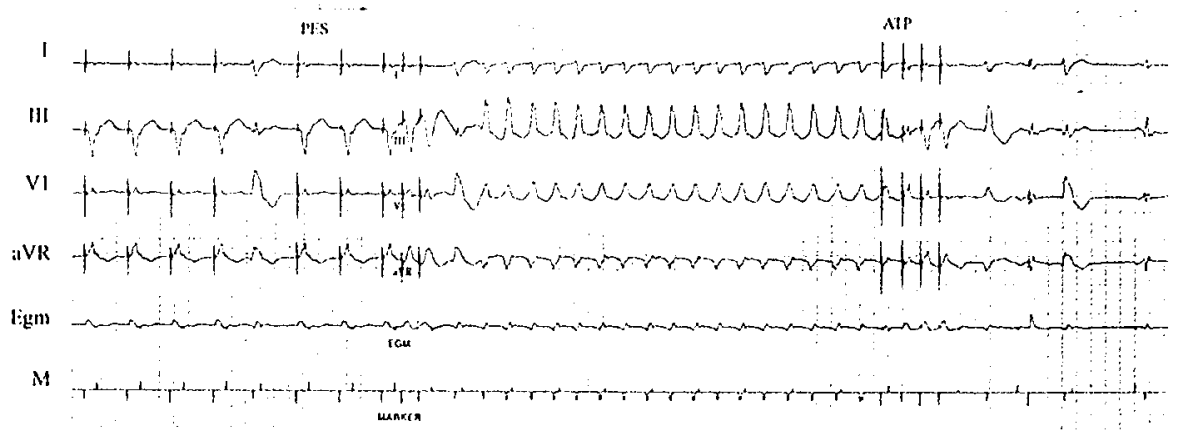

B

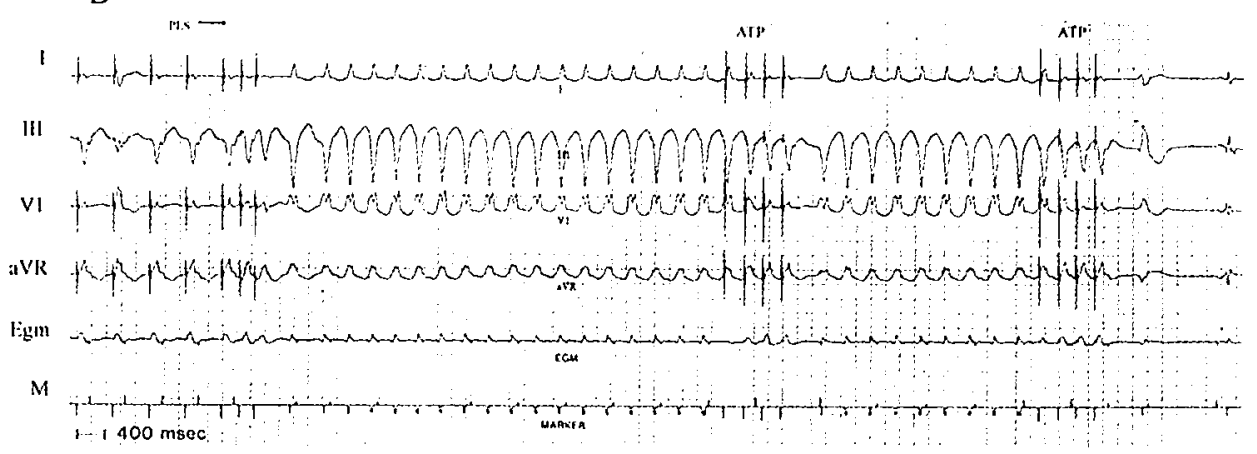

Figure 39. Predischarge testing. Different therapy efficacy in different morphologies of clinical VT in one patient. Tracings show four ECG leads, the wideband $A+V$ Egm and the marker channel registration. The VT with the right axis and RBBB morphology (A) can easily be terminated with the first attempt of ATP. The VT with the left axis and (another) RBBB morphology (B), needs two and more ATP sequences. The difference in response to the pacing-therapies is still minor in this case, both morphologies could successfully be terminated with ATP. 


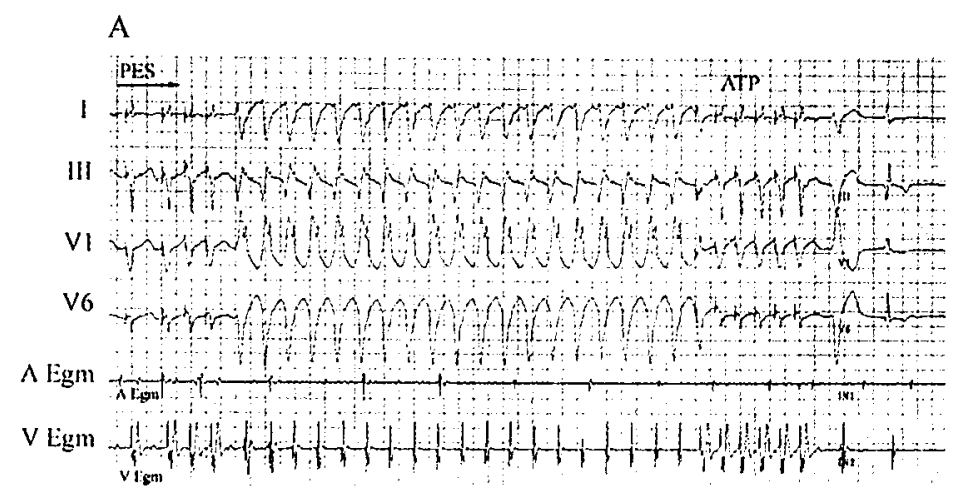

$\mathrm{B}$

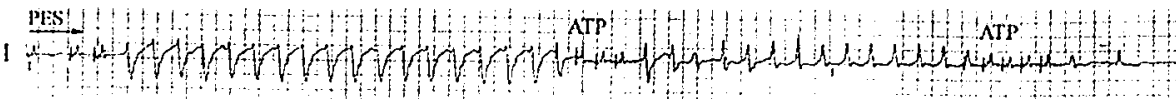

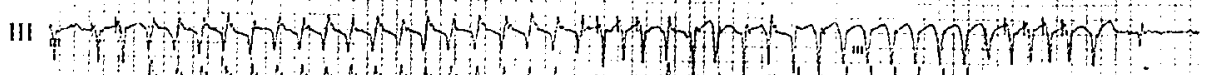

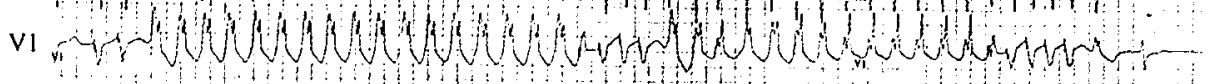

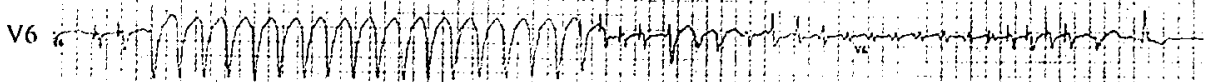
$\triangle \mathrm{Egm} 3 \mathrm{~d}$ f

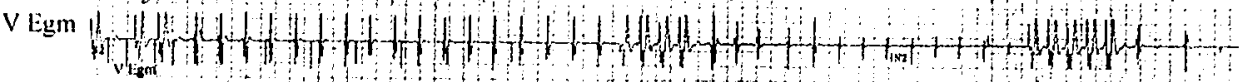
$\mathrm{C}$

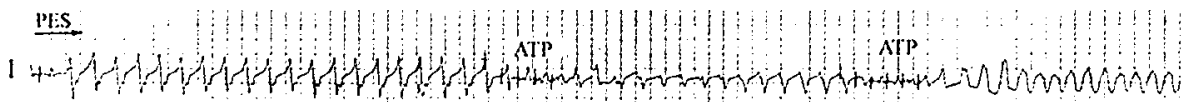

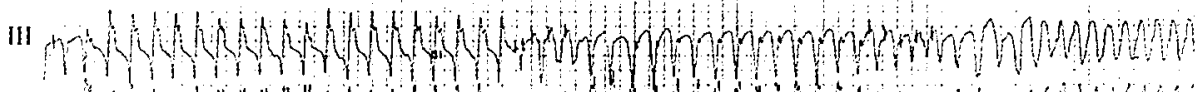

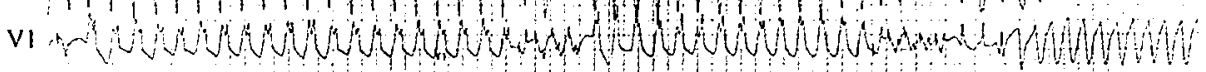

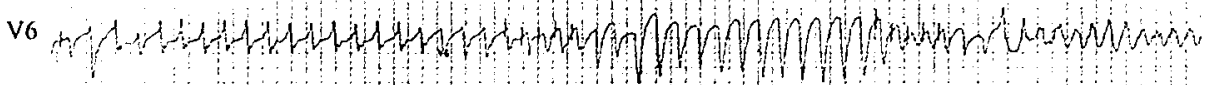

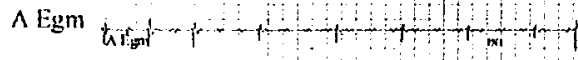

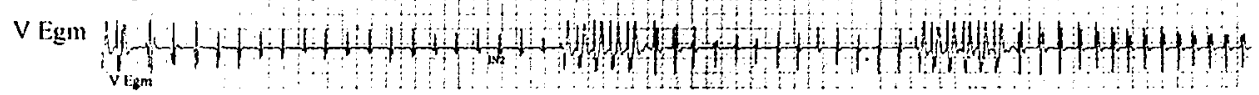
$\therefore 400 \mathrm{msec}$

Figure 40. Predischarge testing. Poor reproducibility of pace-termination of a ventricular arrhythmia. Shown are tracings of four ECG leads, the A Egm and the V Egm registration. The VT in A) and $B$ ) both have the same left axis and RBBB morphology and can be terminated with ATP. The third VT with an intermediate axis and another RBBB morphology is faster and the first sequence of the ATP causes a change in QRS morphology, while the second ATP causes VT acceleration and degeneration into ventricular flutter. 
A

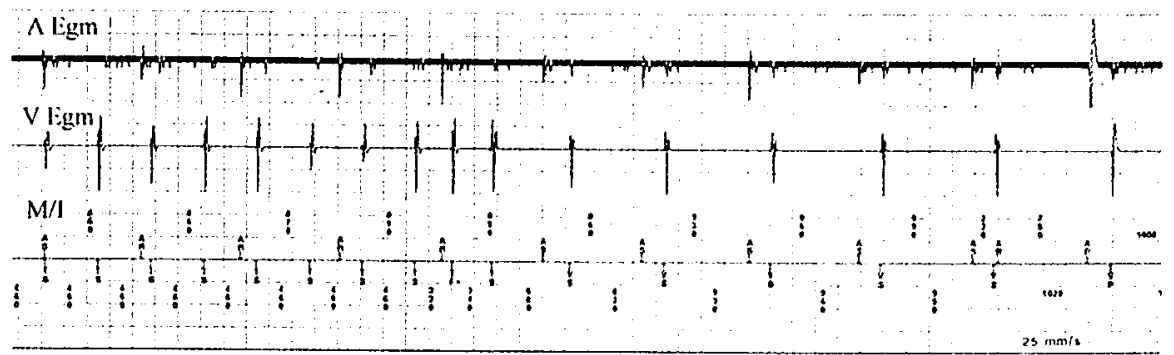

B
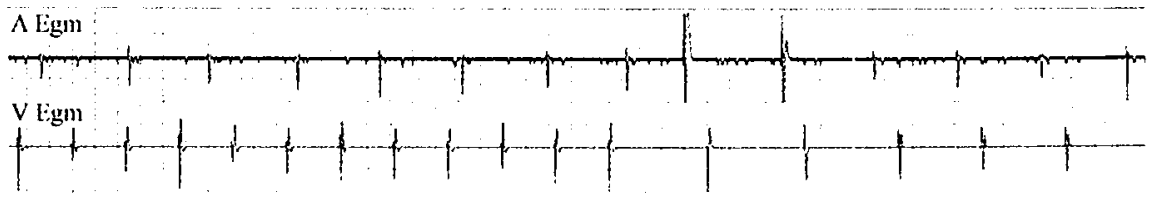

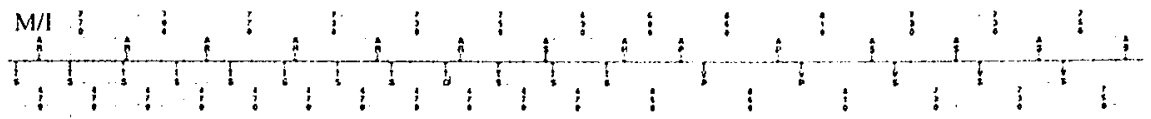

Figure 41. Spontaneous termination of VT. The ICD rhythm recordings show the A Egm, the V Egm and the marker channel with the interval annotation of a spontaneous VT episode. The VT in strip A is terminated by two spontaneous premature beats having a coupling interval of approximately $70 \%$ of the VT cycle length. The VT in strip B terminates without a visible change of the cycle length. (Medtronic 7271 Gem DR).

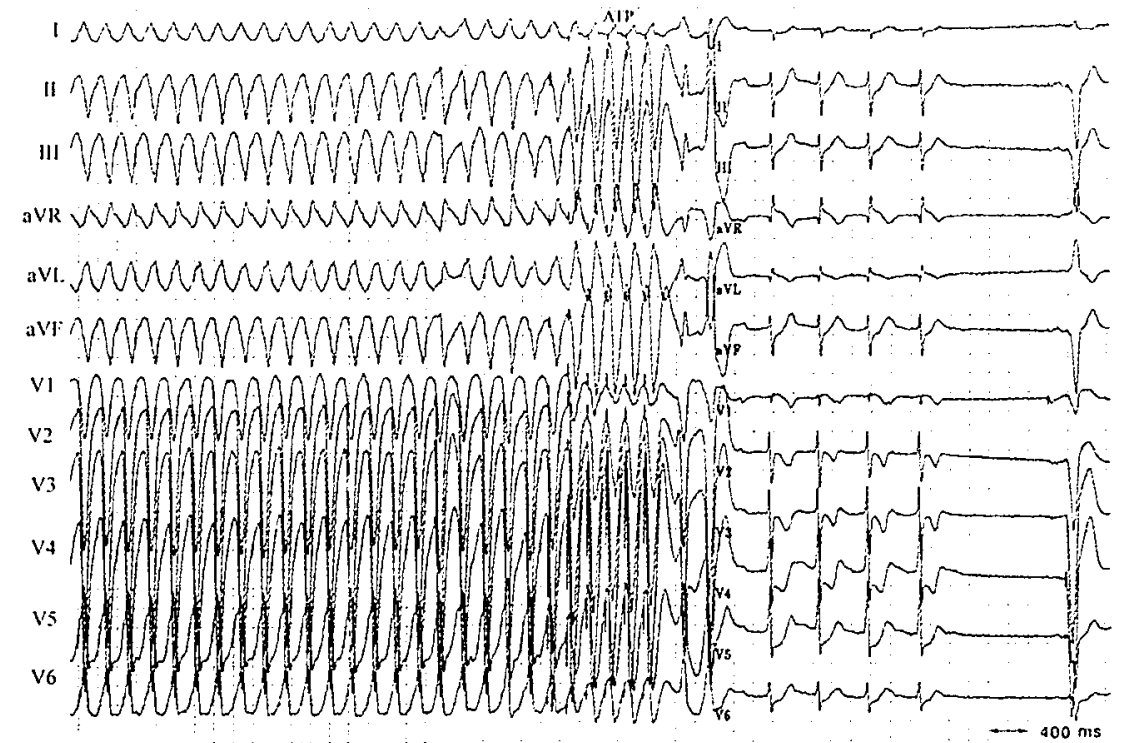

Figure 42. Predischarge testing. ATP termination of a fast VT. Tracings of a 12 lead ECG of a fast VT induced during programmed electrical stimulation. In spite of the short cycle length of the VT and the presence of no other drug than a beta-blocking agent, there is successful pace-termination of the VT. 

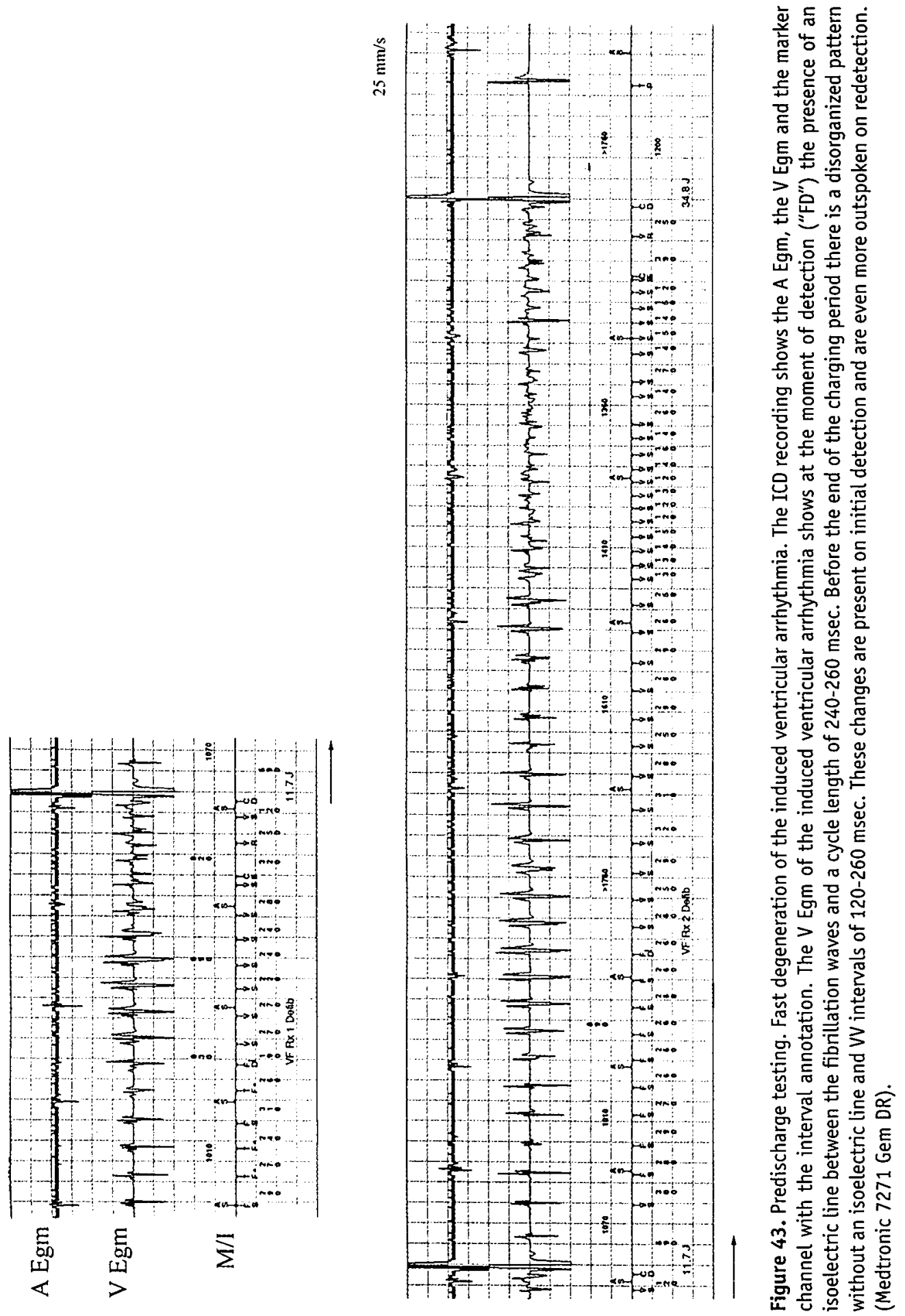


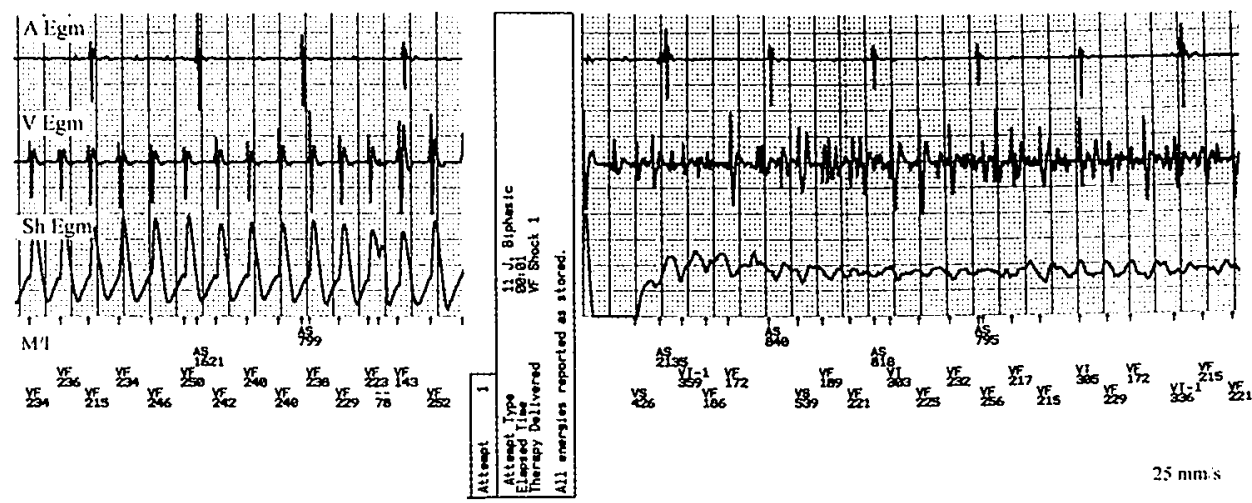

Figure 44. Predischarge testing. Shock induced degeneration of a fast monomorphic VT into VF. The ICD recording shows the A Egm, the V Egm, the Shock Egm, the marker and interval annotation. The $\checkmark$ Egm of the initially induced ventricular arrhythmia shows a fast but organized pattern and the Shock Egm suggests the presence of a fast VT in the VF zone. Following the unsuccessful defibrillation shock the $V$ Egm becomes very fragmented, showing almost continuous activity with hardly any isoelectric line. Many of the fibrillatory waves in V Egm have a small amplitude and some of them are underdetected. (Guidant 1821 Ventak AV II DR).

Figure 45. Predischarge testing. The evaluation of the dual chamber algorithms for detection of supraventricular arrhythmias and the efficacy of device therapies. A) A 12 lead ECG showing an induced AV nodal tachycardia, which is terminated with the commanded (telemetry artifacts) atrial burst therapy (visible in the extremity leads). B) Rhythm recording, including a single lead ECG, the marker channel and the intracardiac V Egm of the same AVN tachycardia as in A. As shown, the automatic detection (and therapy) is suspended and the arrhythmia terminated by the commanded atrial ATP. C) Rhythm recording as in B from a newly induced AV nodal tachycardia. This time the automatic detection and therapies were allowed after the AVN tachycardia had been induced again. Despite the dual chamber arrhythmia detection algorithm being used, the AVN tachycardia was inappropriately classified as VT. This is caused by the fact, that the RP time, in this case the interval between the ventricular sensing moment (early in the QRS) and the retrograde $P$ wave, is slightly longer that $80 \mathrm{msec}$. This RP relation is classified by the ICD as no longer fitting in the category of supraventricular tachycardias with 1:1 AV relation, but a VT with 1:1 retrograde conduction and the programmed VT therapies are applied. The first ATP reduces the AV conduction to 2:1 and the QRS complexes now show aberration. The second ATP results in termination of the arrhythmia. Although the therapy was inappropriate, it was effective in terminating the AVN tachycardia without causing a ventricular proarrhythmia. (Medtronic 7250 Jewel AF). 
A

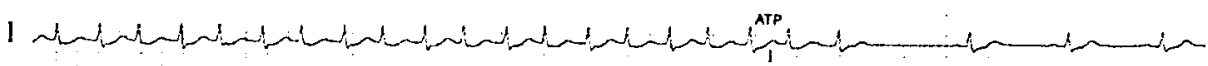

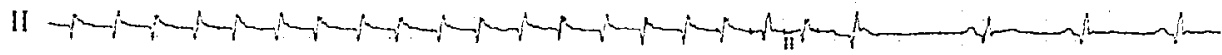

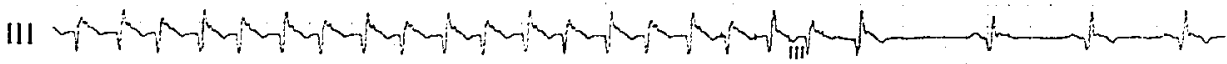
$A V R \rightarrow$ AVL

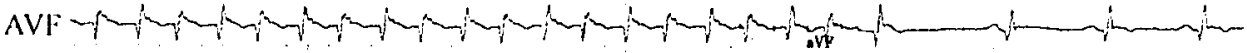
2

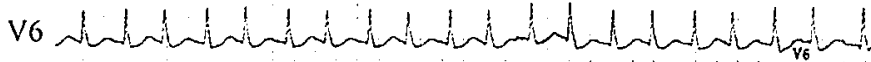
h $\underset{400 \mathrm{~m}}{2}$

B

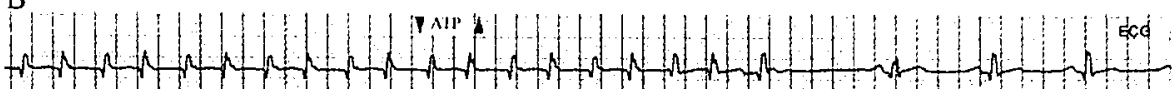

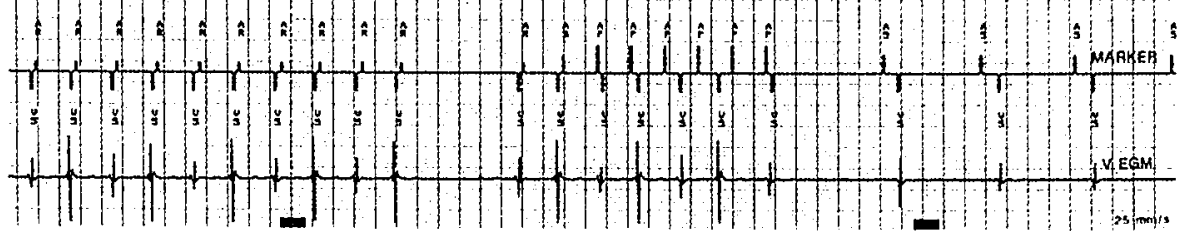

C

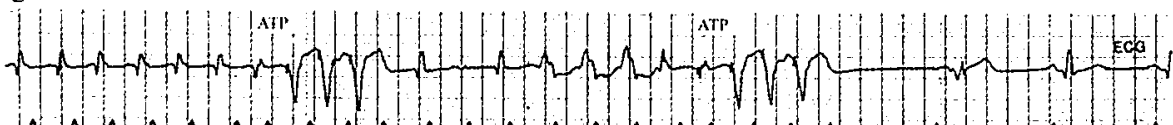

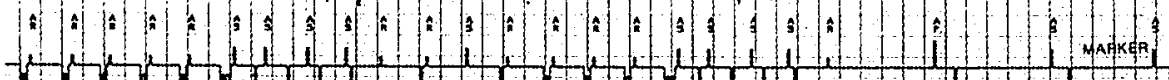

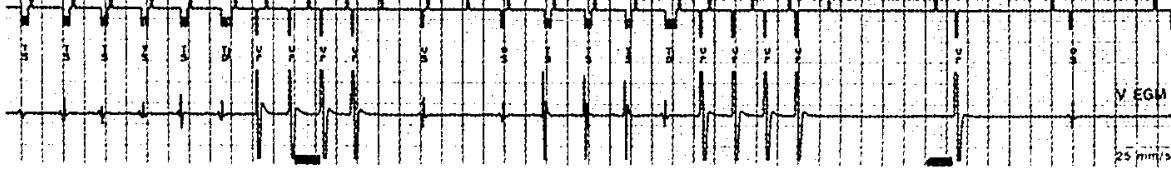




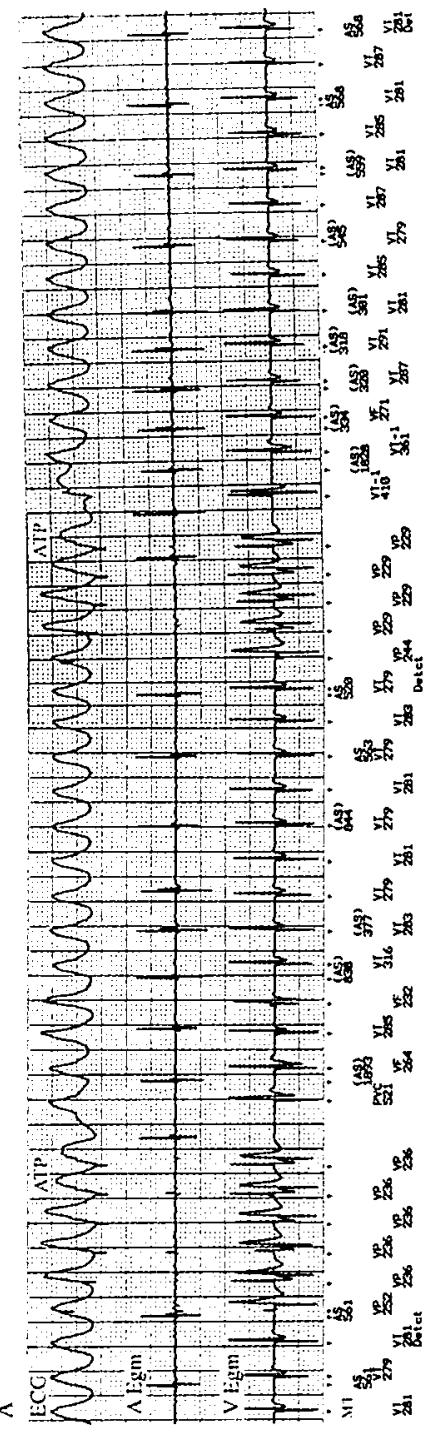

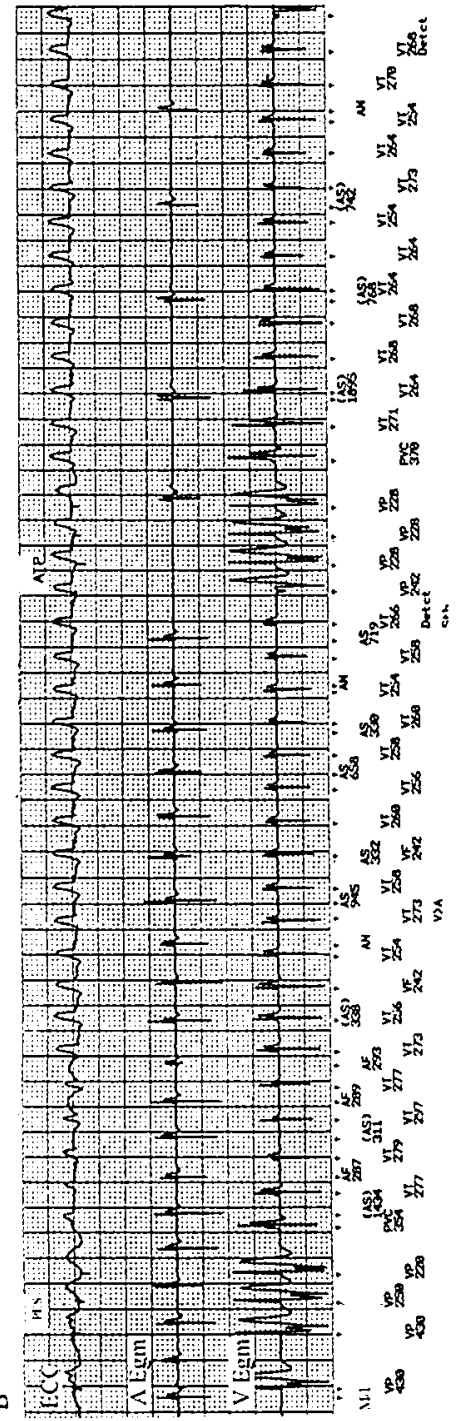

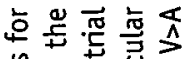

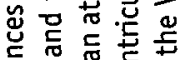

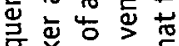

总竞 등믐

ᄃㄹㅇㅇㅇ

:

$5 \pm$.

品导志

든

吉>空㟧定客

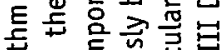

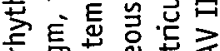
哥衣范文 茂员焉 它.

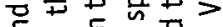
헌든 它出苋范 总品兽莣

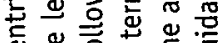

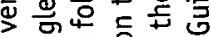
舟. 号战导志

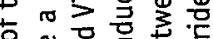

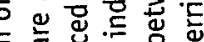
은 胥.

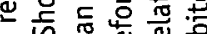
의

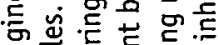

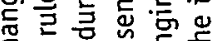
ᄃ

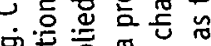

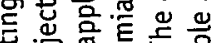

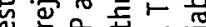

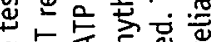

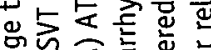

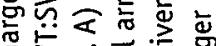
坖宁宁完

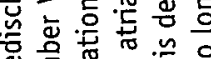
을 웅

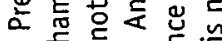

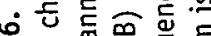

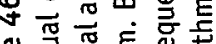

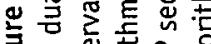

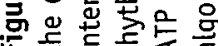


cardioversion induced AF or sinus tachycardia it might be useful to program longer redetection time and to adjust beta-blocking therapy, such as the type of agent or dose. In addition to testing the efficacy of ICD therapies, the accuracy of arrhythmia detection and differentiation, the detection enhancements and the influence of therapies on the detection process are of importance for ICD safety (fig 45). Fast heart rates during ventricular arrhythmias and their electrical therapies might influence the autonomic tone and change the rate of the sinus or atrial rhythm (fig 46). The relation between the atrial and the ventricular rhythm, which may change in the same patient even during one arrhythmia episode is of importance for the functioning of some of the dual chamber detection or redetection algorithms.

After in 1997 ICD therapy was extended with atrial arrhythmia management, study of the atrial arrhythmia became part of the predischarge testing. Atrial arrhythmia induction can be performed using either programmed electrical stimulation or applying 20 msec burst in the atrium (fig 47). The induced atrial arrhythmias are tested for the accuracy of detection algorithms, mode switching operations and the efficacy (and proarrhythmia) of atrial therapies (fig 48).

ICDs with multisite pacing possibilities give the opportunity to test the efficacy of ATP in both the left and the right ventricle and to select which one is most successful.

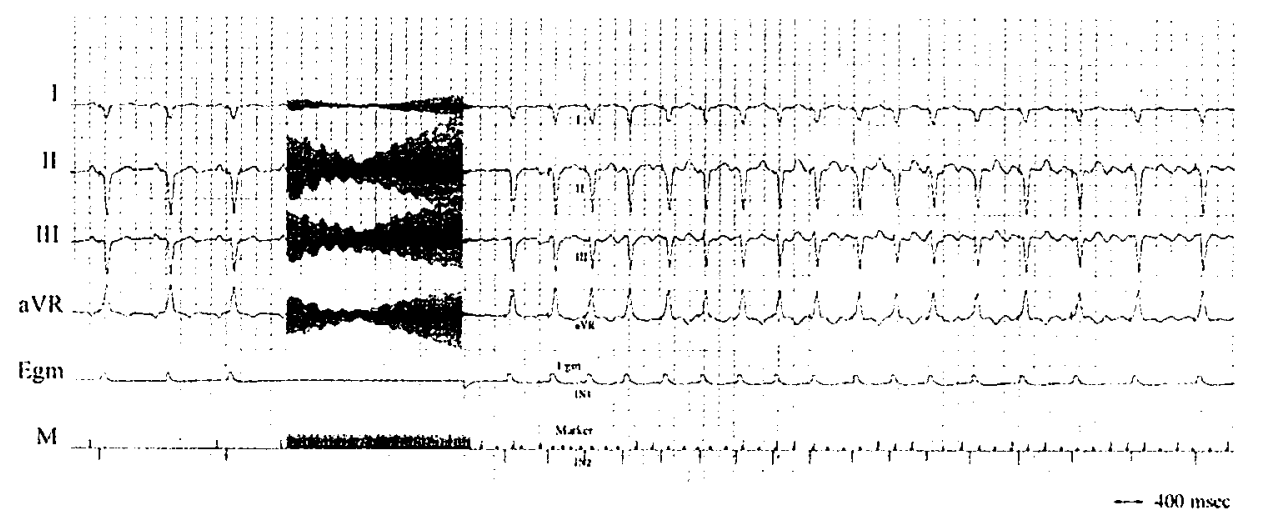

Figure 47. Predischarge testing. Induction of a fast atrial tachycardia using the $20 \mathrm{msec}$ atrial burst to test atrial arrhythmia detection and mode switching algorithms. Shown are four ECG leads, the wideband $A+V$ Egm and the marker channel registration. The atrial tachycardia is properly sensed and the marker channel registration indicates that most arrhythmic events are being detected in the $\mathrm{AF}$ and the AF/AT overlap zone. Only atrial beats within the cross chamber ventricular blanking period are not sensed, which is conform normal function of the device. The mode switching (delta) operation brings the ventricular paced rate from the upper limit down to the lower limit. The temporarily mode switching lower rate cannot be reached, the spontaneous ventricular rate becoming faster that the paced rate. (Medtronic 7250 Jewel AF). 

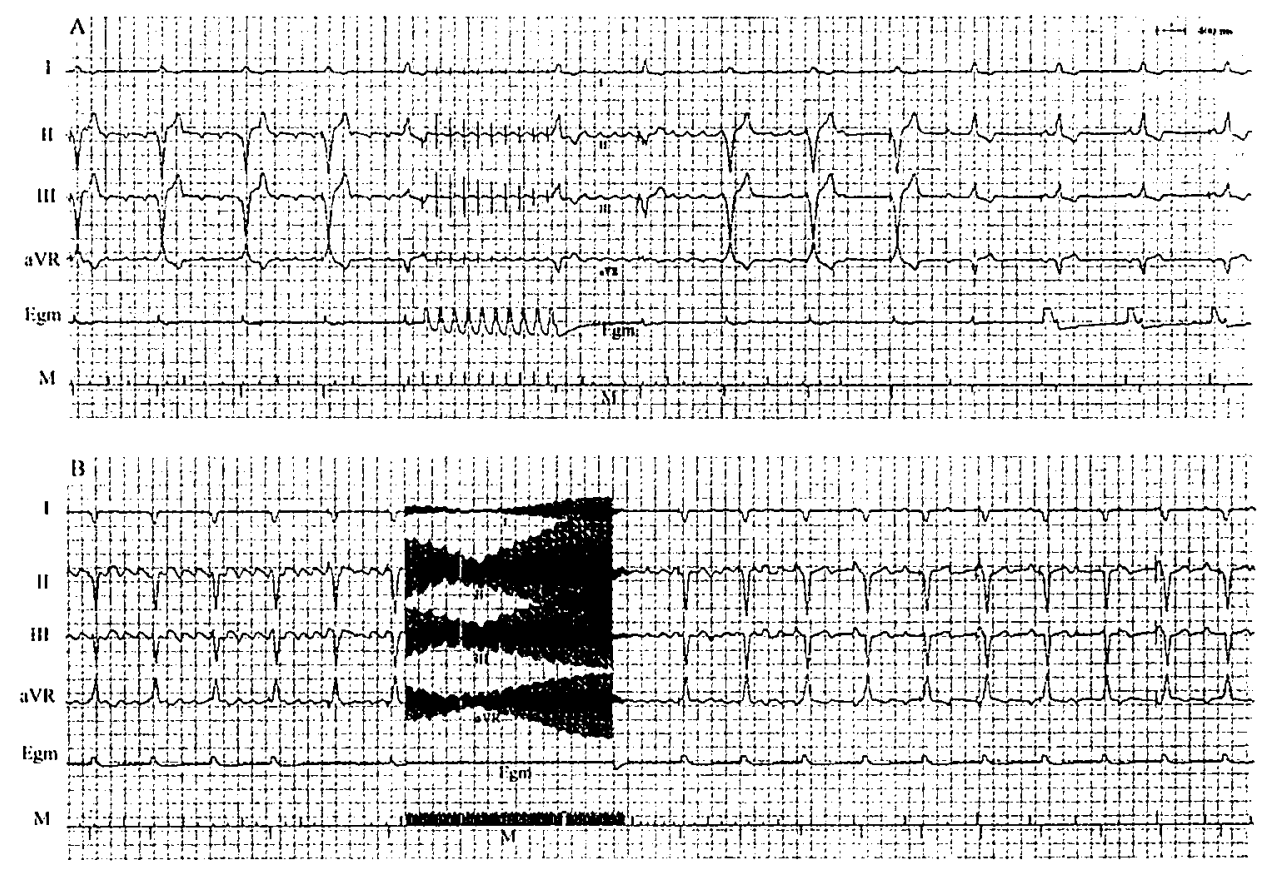

Figure 48. Predischarge testing. Atrial arrhythmia therapies. Shown are four ECG leads, the wideband Egm and the marker channel. Atrial arrhythmias were induced using the $20 \mathrm{msec}$ atrial burst to test the atrial therapies. A) Atrial ATP - Burst + applied for atrial flutter causes a brief change in flutter morphology before termination; B) Atrial $50 \mathrm{~Hz}$ burst applied during 3 seconds results in termination of an atypical flutter, again preceded by brief acceleration. In both cases it takes several RR intervals before the mode switching (fall) operation allows AV resynchronization. (Medtronic 7250 Jewel AF). 


\section{ICD DIAGNOSTICS}

Interrogation of the early devices provided only information on the number of delivered shocks and the battery status. Therefore in the first studies on survival of ICD recipients, impact of ICD therapy could only roughly be estimated, but never overestimated. The shocks accompanied by syncopal symptoms were considered life saving, while the shocks accompanied by other symptoms (palpitations) were considered inappropriate. In 1989 information on the date, the VV intervals and the last applied therapy of the treated ventricular arrhythmias became available. From 1992 the intracardiac ventricular electrograms ( $V$ Egms) could be registered during ventricular arrhythmias (stored Egm) and via telemetry during follow-up tests (real time Egm). The intracardiac Egm became a valuable source of information on clinical aspects of ventricular arrhythmias: the type, the mode of initiation, the response to therapy (fig 49,50). The marker channel annotation became a visible verification of the ventricular sensed and paced events (fig 51), allowing more exact programming of the detection zones and better analysis of troubleshooting. The intracardiac Egms registration was in 1994 further extended by the wideband registration using various Egm sources. The wideband Egm morphology may be helpful in discriminating ventricular and supraventricular rhythms or different morphologies of ventricular arrhythmias (fig 19). At the same time the episode text gave more detailed information on initially detected and redetected arrhythmias, including information on detection enhancement, on consecutive therapies and their results (fig 20). In 1995 interval plots, depicting the relation of the VV intervals versus time, became a useful graphic visualization of rhythm dynamics from before detection until after termination of the arrhythmia (fig 52). With the introduction in 1996 of the dual chamber ICD, arrhythmia documentation became much more complete. Information from the atrial channel including the intervals, the markers, the atrial Egms (A Egm) were added and what is very important, the relation between ventricular and atrial rhythms became visible (fig 23). It allowed more insight in arrhythmia mechanisms and arrhythmia-device interactions. In modern ICDs the arrhythmia logbook gives detailed information about the relation between ventricular and atrial arrhythmias (fig 53) 
[66] Chapter 1

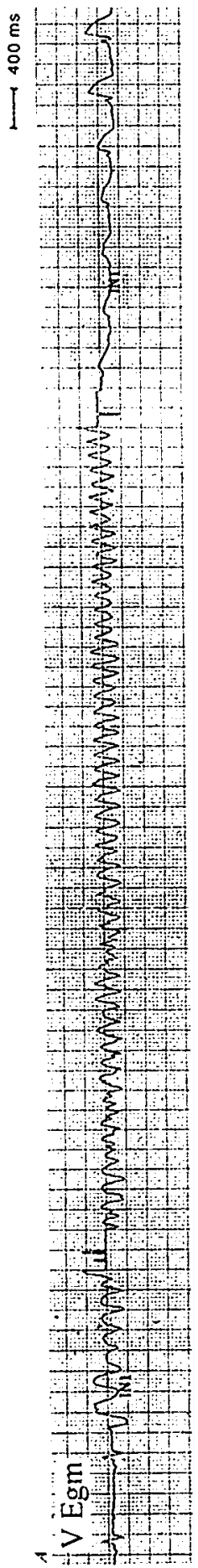

事高

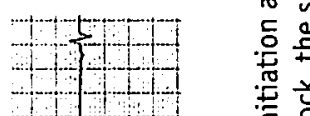

동

.드음

范

음 음

을

点运

a 文 令

空点

营荠

空

돈

额回

这焉

등 는 혿

号岁

$\leq$

톤응

윤

足

突造壳

艛

E气 空

E

兽 흗

恋

苋

芒这.

흥 뭉

至

4 둥

它点

至

营.

不

幽

$F . \subseteq$ 도

نु 峁

号

든들

㞺

嘼

ह

苍究莒

닌 응

守岳

哥号宫 
A
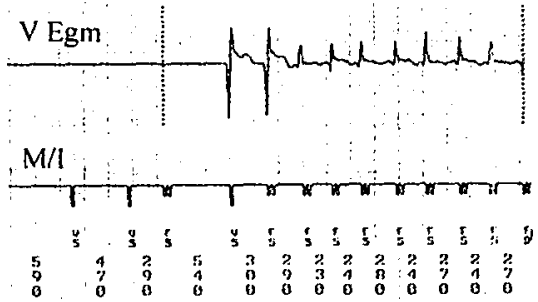

$\mathrm{B}$

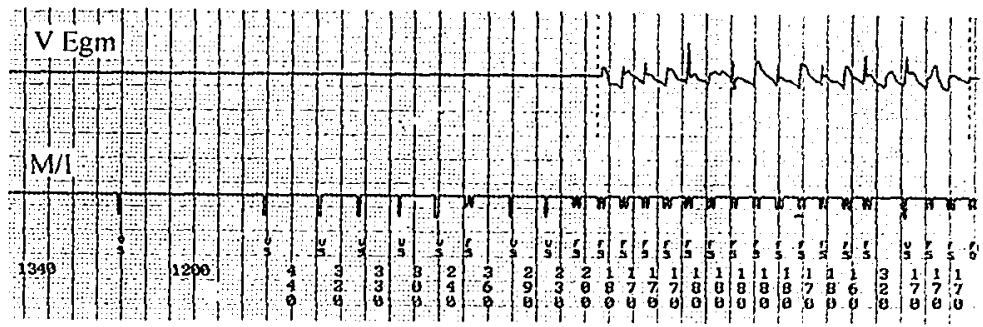

$\mathrm{C}$
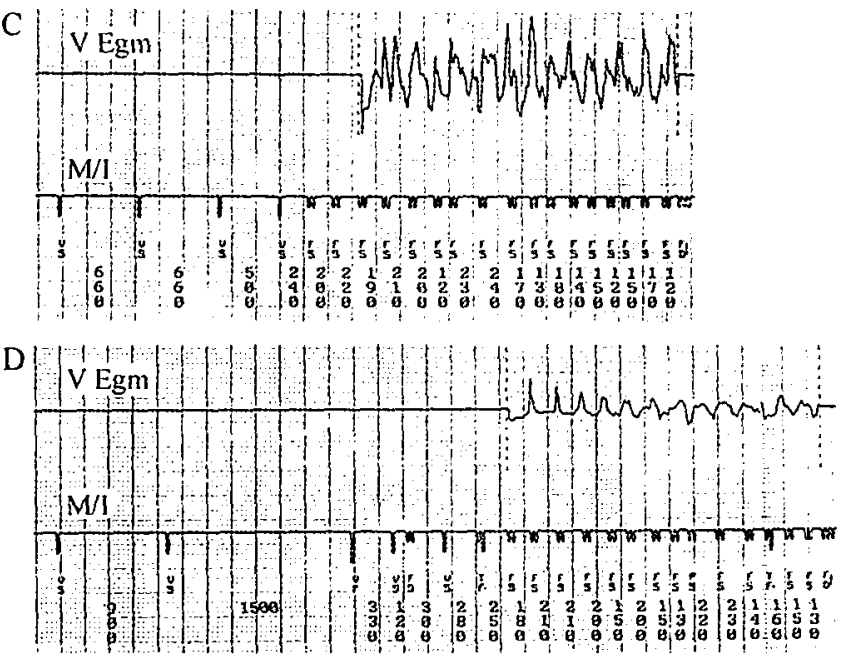

$\mathrm{E}$

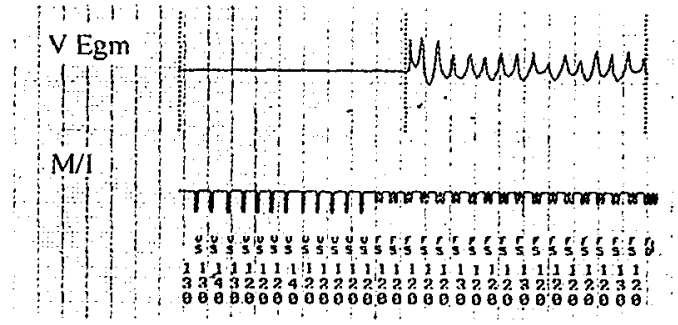

Figure 50. Arrhythmia diagnostics. The intracardiac V Egm of spontaneous (A-D) and induced (E) VF episodes. The Egm and marker registration disclose the variety in ventricular rates preceding the initiation, and the variety of morphology types and cycle lengths. The morphology of the intracardiac V Egm ranges from more "organized" (A) to very "disorganized" $(C, D)$. The cycle lengths of the ventricular arrhythmias vary, in (A) there is a suggestion of a polymorphic VT. The induced VF (E) had an extremely short cycle length less or equal to the ventricular blanking period (120 $\mathrm{msec}$ ). (Medtronic 7219, 7220). 


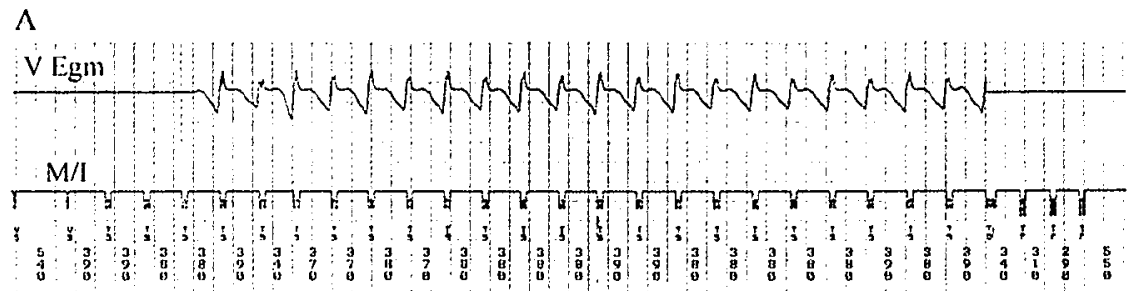

B

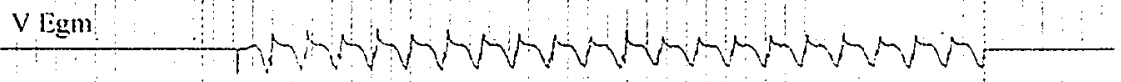

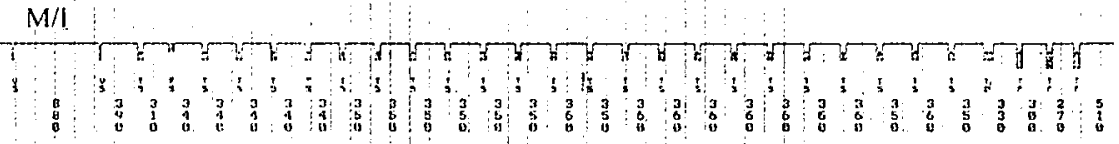

C

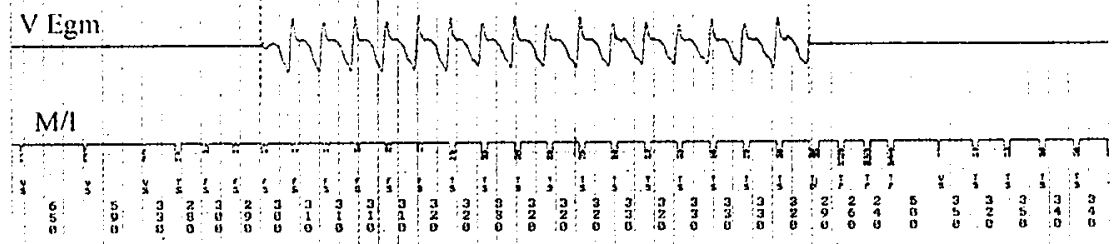

D

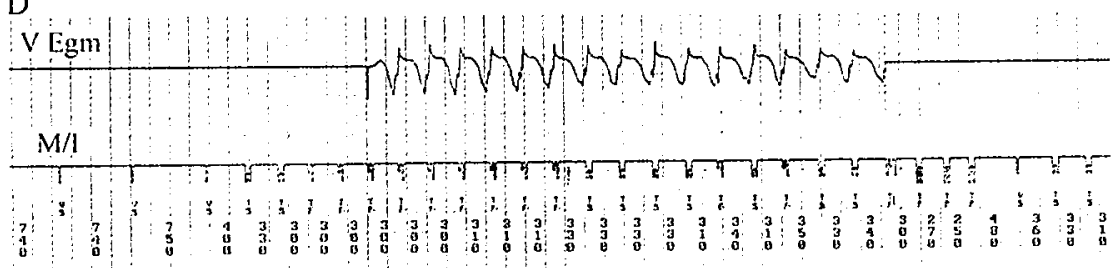

E

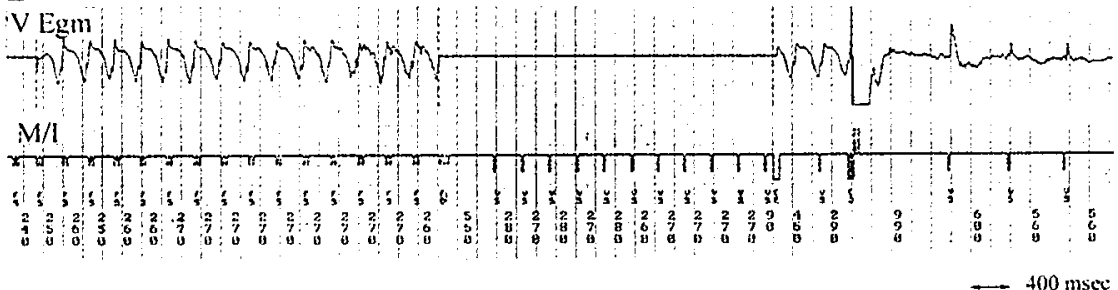

Figure 51. Arrhythmia diagnostics. The availability of the marker channel annotation and intervals allows better programming of the detection zones. Shown are five VT episodes in a patient with an idiopathic dilated cardiomyopathy. The VT has a similar morphology in the intracardiac V Egm, but different cycle lengths. Some of the VT intervals (C, D) are within the VF zone, the VT (D) is detected as fast VT, the VT (E) as VF. The detection of pace-terminable VT within the FVT and VF zone limits the opportunity to apply ATP. The marker and interval annotations are very useful tools allowing fast and exact reprogramming of the detection zones. (Medtronic 7218). 

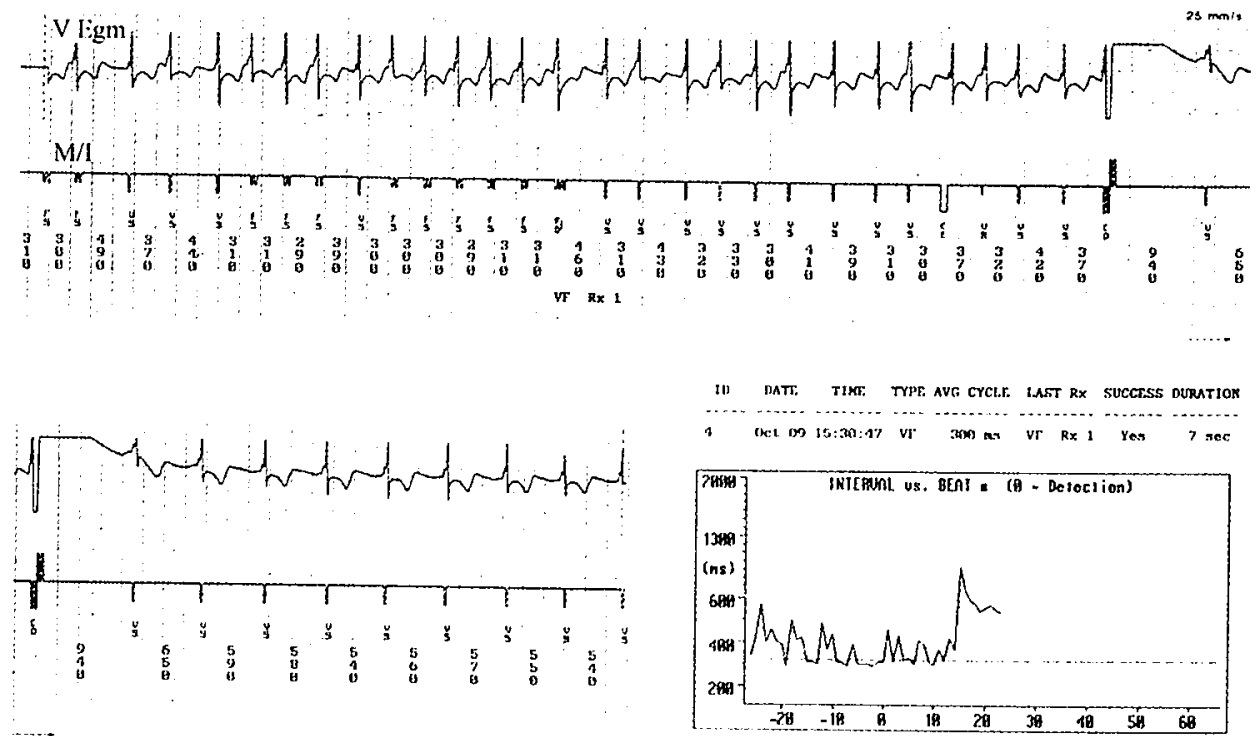

Figure 52. Arrhythmia diagnostics. The VV versus time interval plot. The intracardiac V Egm (continuous registration) is suggestive for $A F$ with a fast ventricular rate inappropriately detected and treated as VF. The interval plot depicts in a very clear manner the rate pattern, in this case the irregularity. In modern ICDs the flashback memory feature records up to 2000 intervals containing both VV and AA intervals prior to the most recent ventricular arrhythmia episode. (Medtronic 7220). 


\section{VENTRICULAR EPISODE SUMMARY REPORT}

Date Interrogated: Nov 26, 1999 11:59:31

Date Last Cleared: Apr 15, 1998 13:02:48

\begin{tabular}{|c|c|c|c|c|c|c|c|c|c|}
\hline ID & DATE & TIME & TYPE & RRME & DIAY & LAST & THE & SUCCESS & DURATION \\
\hline & & & & & & & & & \\
\hline 1026 & Nov 26 & $09: 00: 09$ & VF & 310 & $\mathbf{m s}$ & VF & Rx1 & Yes & $\begin{array}{l}0: 60: 13 \\
0: 00: 11\end{array}$ \\
\hline 4 & Nov 24 & $11: 45: 00$ & VIt & 336 & mS & VI & $\mathrm{R} \times 1$ & es & $00: 00: 07$ \\
\hline 1020 & Nov 21 & $12: 18: 28$ & VT+ + & 350 & ms & VI & $\mathbf{R x t}$ & Yes & $00: 00: 06$ \\
\hline 018 & Nov 20 & $11: 59: 04$ & VT + & 330 & ms & VI & $\mathbf{R} \times \mathbf{1}$ & Yes & $00: 00: 08$ \\
\hline 916 & Nov 14 & $19: 49: 06$ & VT+ & 350 & ms & VI & $\mathbf{R x} \mathbf{1}$ & Yes & $00: 00: 07$ \\
\hline 91.4 & Nov 13 & $10: 11: 22$ & VT+ & 350 & ms & VT & $\mathbf{R x 1}$ & Yes & $00: 00: 07$ \\
\hline 109 & Dec 30 & $56: 28$ & VT & 350 & ms & VT & $\mathbf{k 1}$ & Yes & $00: 00: 1$ \\
\hline
\end{tabular}

\section{ATRIAL EPISODE SUMMARY REPORT}

Date Interrogated: Nov 26, 1999 11:59:31

Date Last Cleared: Apr 15, 1998 13:02:48

\begin{tabular}{|c|c|c|c|c|c|c|}
\hline ID & DATE & TIME & INITIAL & PPMEDIAN & DURATION & LAST Rx/OUTCOME \\
\hline & & - & -- & & -- & \\
\hline 27 & Nov 26 & $09: 60: 38$ & AF & $200 \mathrm{~ms}$ & (Episode & In Progress) \\
\hline 10025 & Nov 24 & $11: 45: 33$ & $\mathbf{A F}$ & $190 \mathrm{~ms}$ & $45: 15: 00$ & AT $R \times 3$ Unknown \\
\hline 20023 & Nov 22 & $11: 29: 12$ & $\mathbf{A F}$ & $200 \mathrm{~ms}$ & $48: 16: 17$ & AT Rx3 Unknown \\
\hline 30021 & Nov 21 & $12: 18: 51$ & $\mathbf{A F}$ & $210 \mathrm{~ms}$ & $23: 10: 08$ & AT Rx3 Unknown \\
\hline 20019 & Nov 20 & $11: 59: 28$ & $\mathrm{AF}$ & $210 \mathrm{~ms}$ & $24: 19: 12$ & AT $\mathrm{R} \times 3$ Unknown \\
\hline 30017 & Nov 14 & $19: 49: 29$ & $\mathrm{AF}$ & $210 \mathrm{~ms}$ & 5 days & AT Rx3 Unknown \\
\hline 30015 & Nov 13 & $10: 11: 47$ & AF & $250 \mathrm{~ms}$ & $33: 37: 35$ & AT Rx3 Unknown \\
\hline$\underline{0013}$ & Nov 12 & $04: 50: 10$ & AT & $250 \mathrm{~ms}$ & $29: 21: 26$ & AF RxI Unknown \\
\hline 10012 & Sep & $07: 19: 51$ & AT & $280 \mathrm{~ms}$ & $03: 29: 29$ & AF $R \times 1$ No Success \\
\hline
\end{tabular}

Figure 53. Arrhythmia diagnostics in the dual chamber device. The use of the arrhythmia logbook to reconstruct the sequence of events in a patient with pump failure, atrial fibrillation and frequent VTs. The atrial episode summary shows that on November 12 an atrial arrhythmia started, with an unsuccessful attempt of termination. One day later a VT is detected. The timeframe of the atrial arrhythmia suggests that it is one ongoing atrial episode, only each new VT detection suspends atrial therapies ("unknown" outcome). Furthermore gradual shortening of the cycle length of both atrial and ventricular arrhythmias is seen. The atrial arrhythmia started with a mean PP interval of $250 \mathrm{msec}$ (shorter than the one 2 months earlier) and was classified as atrial tachycardia. One day later the mean PP interval was the same but the classification was atrial fibrillation, meaning that the arrhythmia has become irregular as well. Furthermore, there is gradual shortening of the PP of the ongoing (but each time newly detected) atrial arrhythmia. The last atrial arrhythmia is redetected after defibrillation therapy for VT, which also became faster and "moved" into the VF zone (last ventricular episode). On September 12 the atrial arrhythmia stopped spontaneously after 3 hours of duration, whereas the present arrhythmia did not stop even after high voltage therapy (for VT detected as VF). Fortunately the "VT+" (dual arrhythmia) responded well to ATP ventricular therapies. (Medtronic 7250 Jewel AF). 


\section{REFERENCES}

1. Nisam S, Barold S: Historical evolution of the automatic implantable cardioverter defibrillator in the treatment of malignant ventricular tachyarrhythmias. In: E.Alt, H.Klein, J.Griffin, (Eds): Implantable Cardioverter Defibrillator. Springer-Verlag 1992; pp 3-10. (With permission of Springer-Verlag).

2. Liberthson $R$, Nagel $E$, et al. Prehospital ventricular defibrillation - prognosis and follow-up course. N Engl J Med 1974; 291:317-321.

3. Baum R, Alvarez H, Cobb L. Survival after resuscitation from out-of-hospital ventricular fibrillation. Circulation 1974; 50:1231-1235.

4. Kastor J. Michel Mirowski and the automatic defibrillator. Am J Card 1989; 15:63 (13) 977-82; and: (15) 1121-1126.

5. Lown B, Axelrod P. Implanted standby defibrillators. Circulation 1972; 46:637-639.

6. Mirowski M, Reid P, Mower M, et al. Termination of malignant ventricular arrhythmias with an implanted automatic defibrillator in human beings. $N$ Engl J Med 1980; 303:322-324.

7. Marchlinski F, Flores B, Buxton A, et al. The automatic implantable cardioverter defibrillator: Efficacy, complications and device failure. Ann Intern Med 1986; 104: 481-488.

8. Reid P, Mirowski $M$, Mower $M$, et al. Clinical evaluation of the internal automatic cardioverter defibrillator in survivors of sudden cardiac death. Am J Cardiol 1983; 51:1608.

9. Echt D, Armstrong D, Schmidt P, et al. Clinical experience, complications and survival in 70 patients with the automatic implantable cardioverter/defibrillator. Circulation 1985;71: 289-296.

10. Kelly P, Cannon D, Garan $\mathrm{H}$, et al. The automatic implantable cardioverter defibrillator: Survival in patients with malignant ventricular arrhythmias. J Am Coll Cardiol 1988; 11 : 1278-1286.

11. Bach S, Barstadt J, Harper N, et al. Initial clinical experience: Endotak $\mathrm{rM}$ implantable transvenous system. J Am Coll Card 1989; 13:65 (A).

12. Brooks R, Garan $H$, Torchiana D, et al. Determinations of successful nonthoracotomy cardioverter-defibrillator implantation: Experience in 101 patients using two different lead systems. J Am Coll Cardiol 1993; 22:1835-1842.

13. Sra J, Natale A, Axtrell K, et al. Experience with two different nonthoracotomy systems for implantable defibrillator in 170 patients. PACE 1994; 17:1741-1750.

14. Zipes D, Roberts D: Results of the international study of the implantable pacemaker cardioverter-defibrillator: A comparison of epicardial and endocardial lead systems. Circulation $1995 ; 92: 59-65$.

15. Kopp D, Blaeman B, Kall J, et al. Predictors of defibrillator energy requirements with nonepicardial lead system. PACE 1995; 18:253-260.

16. Neuzer J, Pitschner H, Huth, et al. Effect of biphasic waveform pulse on endocardial defibrillation efficacy in humans. PACE 1994; 17:207-212.

17. Block M, Hammel D, Docker D, et al. A prospective randomised cross over comparison of mono- and biphasic defibrillator using nonthoracotomy lead configuration in humans. J Cardiovasc Electrophysiol 1994; 5:581-590.

18. Natale S, Sra J, Axtell K, et al. Preliminary experience with a hybrid nonthoracotomy defibrillating system that includes a biphasic device: Comparison with standard monophasic device using the same lead system. J Am Coll Cardiol 1994; 24:406-412. 
19. Tullo N, Saksena S, Krol R, et al. Management of complications associated with a first generation endocardial lead systems for implantable cardioverter defibrillator. Am J Cardiol, 1990; 66: 411-415.

20. Lang D, Heil J, Hahn S, et al. Implantable cardioverter defibrillator lead technology. PACE 1995;18:548-559.

21. Bardy G, Johnson G, Poole J, et al. A simplified single-lead unipolar transvenous cardioversion-defibrillation system. Circulation 1993; 88(2): 1-5.

22. Jordaens $L$, Vertongen $P$, Belleghem $Y$ : A Subcutaneous lead array for implantable cardioverter defibrillators. PACE 1993; 16:1429-1433.

23. Higgins $S$, Alexander D, Kuyper C, et al. The subcutaneous array: A new lead adjunct for the transvenous implantable cardioverter defibrillator system to lower defibrillation thresholds. PACE 1995; 18: 1540-1548.

24. Wood M, Swerdlow C, Olson W. Sensing and arrhythmia detection by implantable devices. In: K.Ellenbogen, G.Kay, B.Wilkoff. Clinical Cardiac Pacing and Defibrillation. W.B. Saunders Company 2000; pp 68-126.

25. Model 7218 Arrhythmia Management Device. Technical Manual. Medtronic, Inc 1994.

26. Ventak AV II DR Implantable Cardioverter Defibrillator. Physician's system manual. Cardiac Pacemakers, Inc 1998.

27. Lavergne T, Daubert J, Chauvin M, et al. Preliminary Clinical Experience with the First Dual Chamber Pacemaker Defibrillator. PACE 1997; 20 (1-II, Cardiostim);182-188.

28. Boecker D, Aliot E, Brachmann J, et al. Benefit of an Enhanced Detection Algorithm in Optimizing Treatment of Slow Ventricular Tachycardia. PACE 2000; 23: 95(A).

29. Israel C, Grönfeld G, Iscolo N, et al. Discrimination Between Ventricular and Supraventricular Tachycardia by Dual Chamber Cardioverter Defibrillators: Importance of the Atrial Sensing Function. PACE 2001; 24:183-190.

30. Jewel AF Arrhythmia Management Device. System reference guide. Medtronic, Inc.1996.

31. Gem DR II Dual Chamber Implantable Cardioverter Defibrillator. System reference guide. Medtronic USA, Inc 1998.

32. Tachos DR Technical Manual, Biotronic GmbH\&Co, 2000.

33. Luceri R: Initial clinical experience with a dual chamber rate responsive implantable cardioverter defibrillator. PACE 2000; (23): 1986-88.

34. Peters $\mathrm{R}$, Zhang $X$, Gold $\mathrm{M}$ : Clinical predictors and efficacy of antitachycardia pacing in patients with implantable cardioverter defibrillator. PACE 2001;24:70-4.

35. Almendral J, Arenal A, Villacastin J, et al. The importance of antitachycardia pacing for patients presenting with ventricular tachycardia. PACE 1993;16:535-9. 
CHAPTER 2

ICD Therapy in Maastricht Academic Hospital 


\section{INTRODUCTION}

The first clinical trial on feasibility, safety and clinical value of an implantable cardioverter defibrillator was completed in 1985 and followed by FDA approval for market release. In the same year the first ICD implantation was performed on December $6^{\text {th }}$ in the Academic Hospital in Maastricht. The concept of ICD therapy as a treatment modality for ventricular arrhythmias underwent dynamic changes in the past 15 years. The most important positive impulse was the fascinating technological development of the system hardware allowing reduction of the implantation risks. The sophistication of algorithms for arrhythmia detection and therapy allowed optimization of the electrical therapies in different types of ventricular and supraventricular arrhythmias. Furthermore, pacing for arrhythmia prevention or for pumpfailure added new therapies to the original ICD concept. On the other hand the use of ICD as treatment for ventricular arrhythmias was hampered by skepticism about advantages of device therapy, the faith in efficacy of other modalities to treat ventricular arrhythmias, the complications of ICD therapy and the high initial costs. In the past 15 years the value of the ICD therapy recognized in many retrospective studies was confirmed in prospective randomized trials $(1,2)$. Now the ICD has become the first choice therapy in an increasing number of patients with different types and etiologies of ventricular arrhythmias.

This chapter describes the demographic and clinical characteristics of patients treated with the ICD therapy in our institution between December 1985 and October 2000. The number of patients and their characteristics refer to their initial implant (excluding replacements), which means that each patient is mentioned only once. The first patient implanted in our center was a 35 years old man with ventricular fibrillation (VF) and an apparently normal heart, survivor of already two episodes of cardiac arrest and two other episodes of unwitnessed syncope. Since then the number of new ICD implantations has been increasing (fig 1), although restricted by budgetary measures. More increase was observed after the introduction of new technologies, like the change from thoracotomy to the nonthoracotomy lead system. Moreover, the outcome of ICD trials discouraged use of other therapies in sudden death survivors [AVID, CASH, The Dutch Study (3-6)], or resulted in expanding ICD indications [MADIT, CIDS, (7-9)]. On the other hand the emergence of new Dutch implantation centers altered the geographic distribution of referred ICD recipients to our center, as it was the case in 2000 .

During the past 15 years 340 patients received an ICD in our institution; 288 male and 52 female patients with a mean age of $56 \pm 14$ (17-77) years. The age distribution of the ICD recipients is shown in fig 2 . 


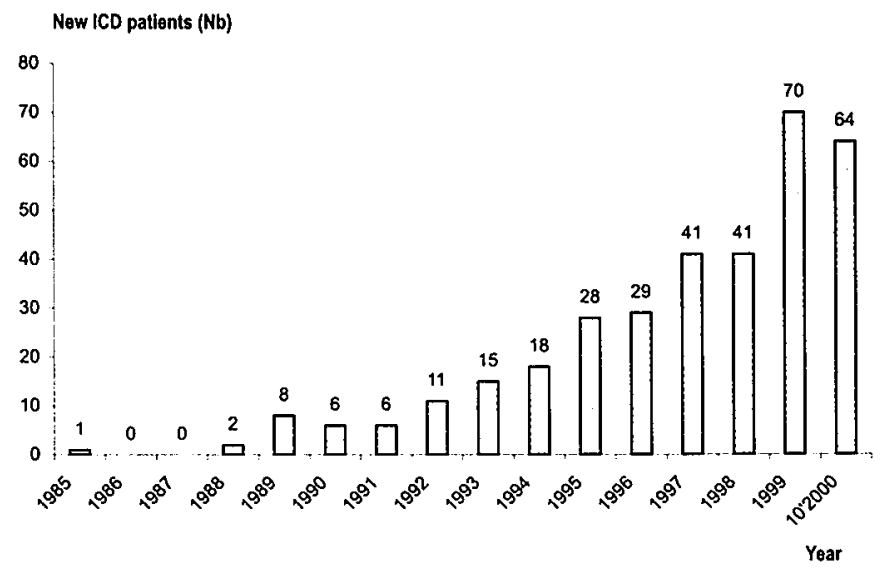

Figure 1. New ICD patients implanted in our center between December 1985 and 0ctober 2000. Shown are increasing numbers of new patients treated with ICD therapy.

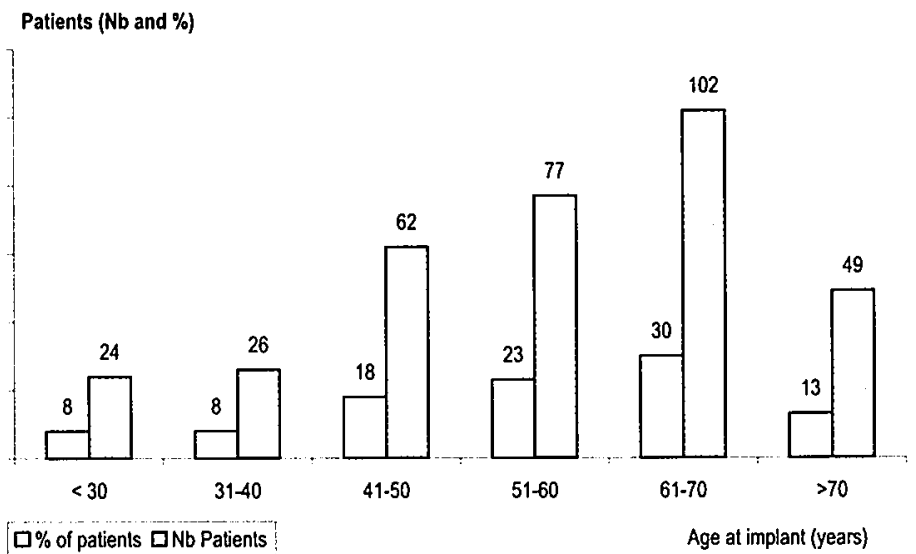

Figure 2. Age of the ICD patient at the time of implantation. The $x$-axis represents the age groups, the $y$-axis the number of patients. The gray bars show the number of patients, the white bars represent the percentage of the whole ICD population.

\section{VENTRICULAR ARRHYTHMIA ETIOLOGY}

The implantation of an ICD is preceded by a work-up, during which the ventricular arrhythmia etiology and possible therapeutic strategies are evaluated. This work-up includes a 12 lead ECG, a chest $x$-ray, echocardiography, exercise testing, Thallium myocardial perfusion scintigraphy in patients with coronary artery disease and in some forms of cardiomyopathy, coronary angiography, and an electrophysiological study. 
Furthermore, a MRI is performed in patients with arrhythmogenic right ventricular dysplasia, in some patients with a poorly understood type of cardiomyopathy and in those with an apparently normal heart. In addition, in patients with a cardiomyopathy, in whom cardiac disease was suspected to be part of a systemic disease, a myocardial biopsy was performed. Patients in whom no abnormalities were found using the above-mentioned diagnostic techniques, have been classified as idiopathic VF. At the present time, this is a diverse group. In some patients disease can be present at the ionic channel level, so they cannot be considered as idiopathic any more. For example patients with the distinct ECG pattern of RBBB and ST elevation in V1-3 have become a separate subgroup. It is likely, that also in other subgroups of patients with idiopathic VF, an explanation for their arrhythmia will eventually be found at the (sub) cellular level. Therefore at this moment, the term "apparently normal heart" is used to refer to the previously used entity idiopathic VF.

Using the described investigations the ventricular arrhythmia is classified as belonging to one of 10 etiologies: coronary artery disease (CAD), dilated cardiomyopathy (DCMP), hypertrophic cardiomyopathy (HCM) valvular heart disease (Valv), hypertensive heart disease (HThd), arrhythmogenic right ventricular dysplasia (ARVD), other forms of cardiomyopathy (CMP-other), long QT syndrome (LQTS), congenital heart disease (congenital), apparently normal heart ("N").

The frequency of these different etiologies is shown in table 1.

With the exception of 4 cases of valvular heart disease, there is no overlap between coronary artery disease and any other etiology. For example, dilated cardiomyopathy was not due to ischemic disease.

The group of CMP-other includes heart disease that could not be classified in any other etiology. To this group belong cardiac (and sometimes systemic) abnormalities such as: sarcoid heart disease (1), Takayashu's arteritis (1), M.Steinert (1), right ventricular cardiomyopathy with minor left ventricular involvement and no features typical for ARVD (2), left and right ventricular dilatation in athletes (2), "segmental myopathy" of the left ventricle (4). The last term describes the presence of one or two isolated segments showing hypokinesia or akinesia, often with normal wall thickness in an otherwise normal left ventricle without evidence of coronary artery disease or a history of a coronary accident.

\section{VENTRICULAR ARRHYTHMIAS BEFORE ICD IMPLANT}

\section{Clinical presentation of ventricular arrhythmia}

The clinical presentation of ventricular arrhythmias before ICD implant varies in severity. Two hundred twenty two patients (65\%) survived cardiac arrest outside (or in) hospital. Syncopal symptoms such as collapse, near-collapse or dizzy spells 
Table 1. Type of Heart Disease in ICD Recipients.

\begin{tabular}{lcc}
\hline Arrhythmia Etiology & Nb of patients & $\%$ Patients \\
\hline Coronary Artery Disease & 226 & $66.5 \%$ \\
Dilated Cardiomyopathy & 31 & $9 \%$ \\
Hypertrophic Cardiomyopathy & 7 & $2 \%$ \\
Arrhythmogenic Right Ventricular Dysplasia & 13 & $4 \%$ \\
Valvular Heart Disease & 13 & $4 \%$ \\
Hypertensive Heart Disease & 11 & $3 \%$ \\
CMP- other & 11 & $3 \%$ \\
Congenital Heart Disease & 3 & $1 \%$ \\
Long QT Syndrome & 2 & $0.5 \%$ \\
Apparently Normal Heart & 23 & $7 \%$ \\
Total & 340 & $100 \%$ \\
\hline
\end{tabular}

occurred in $100(30 \%)$ patients. Nonsyncopal symptoms, such as palpitations, dyspnoe or angina pectoris during their ventricular arrhythmia were present in $16(4,5 \%)$ patients. In 95 (28\%) of patients such nonsyncopal symptoms were present before they presented with more severe symptoms, such as cardiac arrest or syncope, which eventually became the ICD indication.

Two patients, one with ARVD and one with HCM, never experienced any arrhythmia related symptoms, but the ICD was implanted because of a family history of sudden death at a young age (below 40 years) in two or more family members.

\section{Ventricular arrhythmia documentation before ICD implant}

Ventricular fibrillation was the presenting ventricular arrhythmia in 162 (47\%) patients. Sustained ventricular tachycardia (VT) was documented as the presenting ventricular arrhythmia in 87 (26\%) patients (in 4 polymorphic). Both VF and VT were documented in $56(17 \%)$ patients. Nonsustained ventricular tachycardia (nvVT) was observed in 28 patients. In $52 \%$ of patients with VF and in a similar proportion of patients with VT, nsVT was documented as well.

In 7 patients no ventricular arrhythmia was documented. Five of them experienced unmonitored syncope and in two patients the ICD was implanted prophylactically because of a family history of sudden death at young age.

\section{Inducibility of the ventricular arrhythmia}

Programmed electrical stimulation was performed in 315 patients. In 243 (71\%) of them resulting in the induction of at least one type of ventricular arrhythmia. In 72 (21\%) patients no ventricular arrhythmia could be induced. Among the inducible patients only one type of arrhythmia was induced in 192 patients: VF in 22, VT in 127 
and nonsustained VTs in 43 patients. In 28 patients both VT and VF and in 23 patients both sustained and nonsustained VT could be induced.

\section{CARDIAC FUNCTION AND FUNCTIONAL CLASS AT THE TIME OF THE PRESENTING ARRHYTHMIA}

Left ventricular ejection fraction as determined by echocardiography had a mean value of $35 \pm 13(12-69) \%$ for the whole group. The LVEF was $<30 \%$ in $124(37 \%)$ patients, $30-40 \%$ in $96(28 \%)$ patients and $>40 \%$ in $120(35 \%)$ patients. In $70 \%$ of patients the left ventricle was moderately dilated, the end-diastolic dimension being $>$ $55 \mathrm{~mm}$. In $12 \%$ of patients the left ventricle was severely dilated, with the end-diastolic dimension being $\geq 70 \mathrm{~mm}$.

Since in $226(66 \%)$ cases coronary artery disease was the etiology of the arrhythmia, a scar of an old infarction was the most frequently observed structural cardiac abnormality. The infarction was localized in the anterior wall in 88 (39\%) patients, in the inferior or posterior wall in $95(42 \%)$ patients and in both the anterior and inferior wall in $40(18 \%)$ patients. In $3(1 \%)$ patients no scar could be localized.

In most patients with coronary artery disease, multivessel disease was present: in 113 (50\%) three vessel and in $42(19 \%)$ patients two vessel disease. In $155(68 \%)$ patients not-infarct related vessels were also diseased. Myocardial perfusion scintigraphy showed evidence of ischemia in $37 \%$ of those patients.

The 114 patients belonging to one of the eight other cardiac disease groups showed cardiac abnormalities typical for their etiology. More frequently than in the coronary heart disease subgroup, these patients showed right ventricular abnormalities, mainly an increased diameter. This was the case in 44/114 (39\%) patients, while only 13 of these patients had typical arrhythmogenic right ventricular dysplasia.

The haemodynamic measurements at the time of evaluation before ICD implantation indicated impaired cardiac function in many patients. 84 (25\%) patients had increased left ventricular end-diastolic pressures and $77(23 \%)$ patients increased right-sided pressures. The exact numbers of patients with impaired haemodynamics is probably underestimated, because most of the patients were referred from other hospitals, and transferred after they were already stabilized.

The NYHA functional class before implantation was 1 in $119(35 \%)$ patients, 2 in 129 (38\%) patients, 3 in $86(25 \%)$ patients and 4 in $6(2 \%)$ patients. 


\section{ICD IMPLANT PROCEDURE}

The ICD system was implanted using a thoracotomy approach in $26(8 \%)$ patients, a non-thoracotomy (partially subcutaneous) approach in $27(8 \%)$ patients and a complete transvenous approach in 287 (84\%) patients.

$158(46 \%)$ patients were implanted with a single chamber and $182(54 \%)$ patients with a dual chamber device.

A concomitant revascularisation procedure was performed in $64(19 \%)$ patients. In 44 surgically and in 20 using coronary angioplasty. When the thoracotomy approach was still used, additional cardiac surgery was performed in the same session as the ICD implant. At present, both procedures are performed on two occasions.

\section{VENTRICULAR ARRHYTHMIAS FOLLOWING ICD IMPLANTATION}

\section{Arrrhythmia recurrences documented by ICD}

Following implantation 3950 ventricular arrhythmia episodes were documented by the ICD in 123 patients, which is $36.2 \%$ of patients receiving an ICD. Using the device diagnostics the type of recurrent ventricular arrhythmia could be classified as VT in 3599 (91\%) episodes, VF or polymorphic ventricular tachycardia in $166(4 \%)$ episodes, a supraventricular tachycardia in $177(4.8 \%)$ episodes and ventricular oversensing in 8 (0.2\%)episodes.

Ventricular arrhythmia recurrences after ICD implant differed in relation to the presenting arrhythmia before ICD implant. Ventricular arrhythmia recurrences occurred in 45/56 (80\%) patients with both VF and VT, in 43/87 (49\%) patients with VT, in $30 / 162(18 \%)$ patients with VF only, in 4/28 (14\%) patients with nonsustained VT and in $1 / 7(14 \%)$ patient with unmonitored syncope.

The ventricular arrhythmia recurrences after ICD implant were different also for patients with different arrhythmia etiologies. As shown in figure 3 the recurrences occurred most often in patients with a dilated cardiomyopathy, coronary artery disease and arrhythmogenic right ventricle dysplasia.

\section{Device therapy for recurrent ventricular arrhythmia}

The detected arrhythmias could be terminated with antitachycardia pacing in 2905 (74\%) episodes. Cardioversion or a defibrillation shock was required in $838(21 \%)$ episodes. The arrhythmia stopped spontaneously in 144 (3.5\%) episodes. Therapy was withheld in $63(1.5 \%)$ episodes because of non-sustained arrhythmia.

Among the 838 ventricular arrhythmias terminated with high voltage therapy 580 occurred in the setting of episodes of electrical instability (repetitive shortly spaced arrhythmia episodes) and 258 during normally distributed single episodes. 


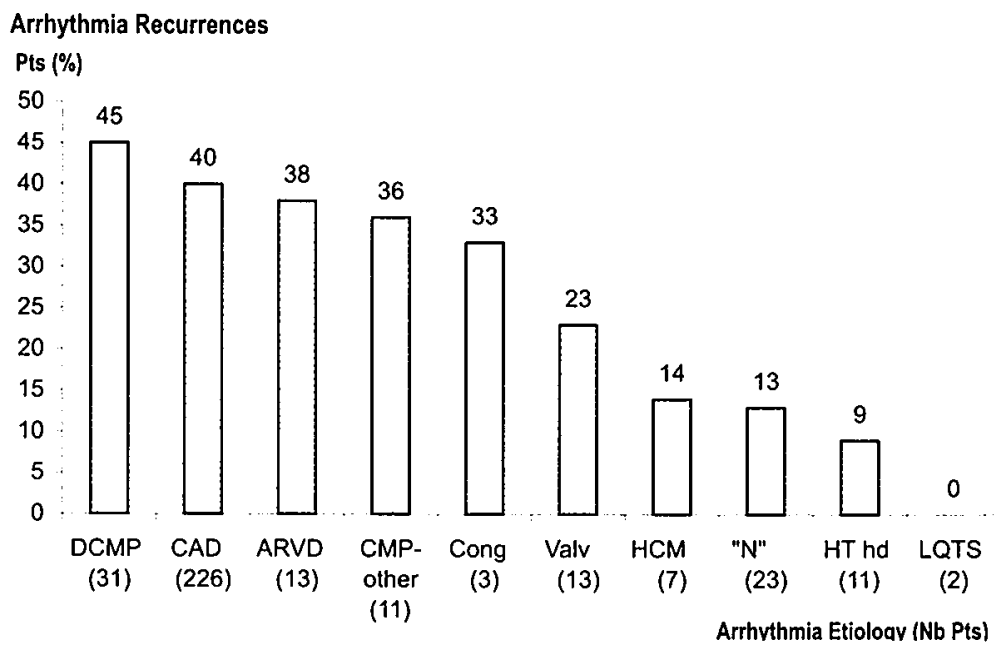

Figure 3. Ventricular arrhythmia recurrences in ICD patients with different types of heart disease. The $x$-axis represents the various etiology groups, the $y$-axis the percentage of patients within these groups with ventricular arrhythmia recurrences after ICD implant.

\section{Symptoms of recurrent arrhythmias}

The arrhythmias and their therapies were asymptomatic in 2262 (57.5\%) episodes, accompanied by nonsyncopal symptoms in 1545 (39\%) and syncopal symptoms in 143 $(3.5 \%)$ instances.

\section{ICD POPULATION DYNAMICS}

The outcome of studies on the role of ICD in the management of ventricular arrhythmia patients together with the advances in ICD technology changed not only the number of implants but also the characteristics of ICD patients. In the thoracotomy era, the ICD recipient was in several ways different from the one currently receiving a device. A typical patient from the eighties was a person with a documented lethal ventricular arrhythmia, usually in the setting of a previous myocardial infarction, in whom at least one modality of secondary arrhythmia prevention failed before implantation of the ICD. The ventricular arrhythmia had to be documented and the symptoms were aborted sudden death. The initial therapy could have been antiarrhythmic drugs, aneurysmectomy, and surgical VT ablation alone or in combination with $C A B G$. The underlying heart disease was of significant importance, but the patient had to be in a physical condition that would allow thoracotomy with an acceptable perioperative risk. The age of a patient was usually between 40-60 years with a reasonable life expectancy. Elderly patients were considered to be at too 


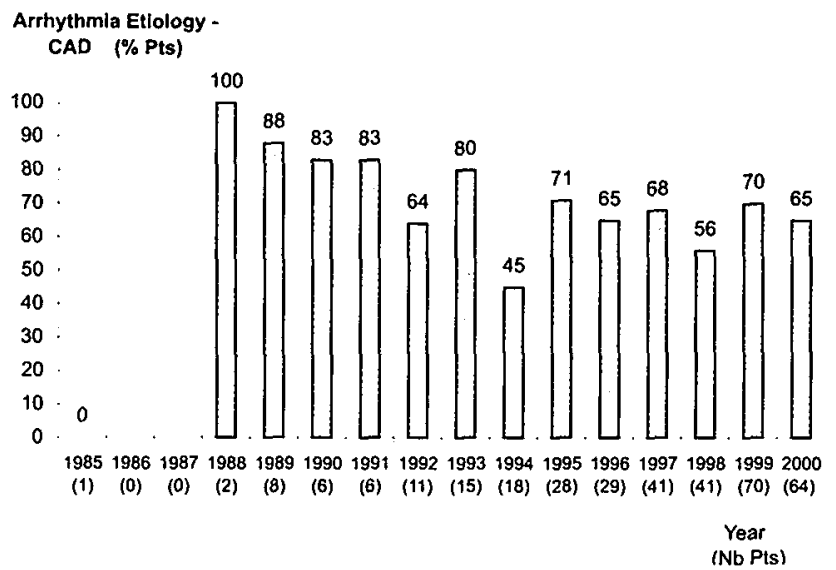

Figure 4. Changes in ventricular arrhythmia etiology in ICD patients. The $x$-axis represent year of initial ICD implantation; the $y$-axis the contribution (in percentages) of patients with coronary artery disease. The number of patients with an arrhythmia etiology other than coronary artery disease is increasing among ICD recipients.

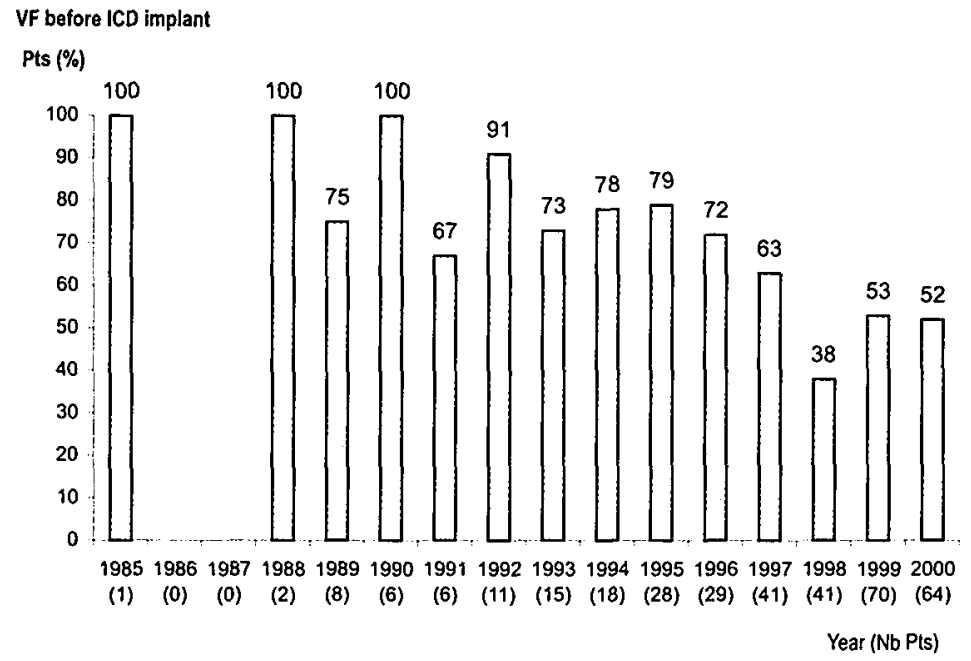

Figure 5. Changes in severity of the presenting arrhythmia in ICD patients. The $x$-axis shows the year of the initial ICD implantation; the $y$-axis the contribution of $V F$ (in percentages) among all types of presenting arrhythmias. The number of patients with other types of arrhythmias than VF is increasing among ICD recipients. 


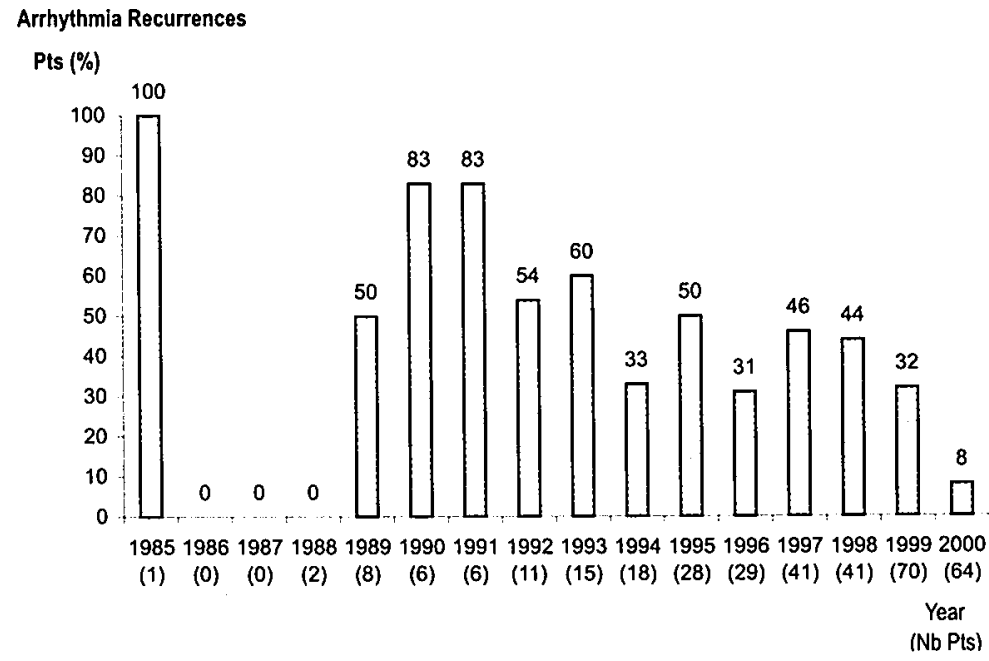

Figure 6. Ventricular arrhythmia recurrences in ICD patients implanted in the past 15 years in our center. The $x$-axis represents the year of initial implant; the $y$-axis the percentage of patients with ventricular arrhythmia recurrences after ICD implantation. The follow-up period becomes gradually shorter which may falsely suggest an apparent decrease in the number of patients having recurrences of a ventricular arrhythmia. Some patients have their first recurrence many years after the ICD implantation.

much risk for perioperative mortality and morbidity. The operative intervention and possible ICD therapies were considered to be too much of a burden in the adolescent patient or child.

At present patients with ventricular arrhythmias of other etiologies are being protected by the ICD therapy against the risk of sudden death. Therefore the contribution of coronary artery disease among the different arrhythmia etiologies has been decreasing (fig 4). Also the number of patients with VF as the presenting arrhythmia is decreasing (fig 5).

The ventricular arrhythmias recurrences in ICD patients implanted in the past 15 years related to the time after the implantation are shown in fig 6 .

Currently the age of the patients varies from below 18 to above 75 years. Their cardiac function differs from very poor (some are waiting for a transplant procedure) to normal. For many patients with a ventricular arrhythmia the ICD has become the therapy of choice.

Current indications for ICD therapy are presented in table 2 and are based on the ACC/AHA "Guidelines for Implantation of Cardiac Pacemakers and Antiarrhythmia Devices" in 1998 (10). The recommendations for indications for ICD therapy are expressed in the standard ACC/AHA format of class I, II, III indications. Class I includes conditions for which there is evidence and/or general agreement that the 
Table 2. Indications for ICD Therapy*.

Class I

1. Cardiac arrest due to VF or VT not due to a transient or reversible cause.

2. Spontaneous sustained VT.

3. Syncope of undetermined origin with clinically relevant, hemodynamically significant sustained VT or VF induced at electrophysiological study when drug therapy is ineffective, not tolerated, or not preferred.

4. Nonsustained VT with coronary disease, prior MI, LV dysfunction, and inducible VF or sustained VT at electrophysiological study that is not suppressible by a Class I antiarrhythmic drug.

\section{Class IIa}

None.

\section{Class IIb}

1. Cardiac arrest presumed to be due to VF when electrophysiological testing is precluded by other medical conditions.

2. Severe symptoms attributable to sustained ventricular tachyarrhythmias while awaiting cardiac transplantation.

3. Familial or inherited conditions with a high risk for life-threatening ventricular tachyarrhythmias such as long QT syndrome or hypertrophic cardiomyopathy.

4. Nonsustained VT with coronary artery disease, prior MI, and LV dysfunction, and inducible sustained VT or VF at electrophysiological study.

5. Recurrent syncope of undetermined etiology in the presence of ventricular dysfunction and inducible ventricular arrhythmias at electrophysiological study when other causes of syncope have been excluded.

\section{Class III}

1. Syncope of undetermined cause in a patient without inducible ventricular tachyarrhythmias.

2. Incessant VT or VF.

3. VF or VT resulting from arrhythmias amenable to surgical or catheter ablation; for example, atrial arrhythmias associated with the Wolff-Parkinson-White syndrome, right ventricular outflow tract VT, idiopathic left ventricular tachycardia, or fascicular VT.

4. Ventricular tachyarrhythmias due to a transient or reversible disorder (eg, AMI, electrolyte imbalance, drugs, trauma).

5. Significant psychiatric illnesses that may be aggravated by device implantation or may preclude systematic follow-up.

6. Terminal illnesses with projected life expectancy 6 months.

7. Patients with coronary artery disease with $\mathrm{LV}$ dysfunction and prolonged QRS duration in the absence of spontaneous or inducible sustained or nonsustained VT who are undergoing coronary bypass surgery.

8. NYHA Class IV drug-refractory congestive heart failure in patients who are not candidates for cardiac transplantation.

* Guidelines for Implantation of Cardiac Pacemakers and Antiarrhythmia Devices: Executive Summary. A Report of the American College of Cardiology/American Heart Association Task Force, 1998. 
ICD therapy is beneficial, useful and effective. Class II includes conditions for which there is conflicting evidence and/or divergence of opinion about the usefulness/efficacy of ICD therapy. Weight of evidence/opinions is in favor of usefulness/efficacy in class IIa, and is less well established in class IIb. Class III includes conditions for which there is evidence and/or general agreement that the ICD therapy is not useful/effective and in some cases may be harmful.

During follow-up 30 patients were referred for control to another ICD center and 24 patients died. The cause of death was cardiac in 20 patients, non-cardiac in 3 patients, and sudden but unknown in 1 patient. Cardiac death was arrhythmic and occurred in hospital in 3 patients during a period of electrical instability, and was nonarrhytmic in 17 patients. The nonarrhytmic death was due to severe ischemia and myocardial infarction in 4 patients, pumpfailure in 8 patients, a combination of both ischemia and electromechanical dissociation in 3 patients and during a complicated course after cardiac transplantation in 1 patient. The noncardiac deaths were due to pneumonia in 2 patients and cancer in 1 patient.

Seven patients underwent a successful heart transplant procedure.

\section{REFERENCES}

1. Cappato R. Secondary prevention of sudden death: the Dutch Study, the Antiarrhythmics Versus Implantable Defibrillator Trial, the Cardiac Arrest Study of Hamburg, and the Canadian Implantable Defibrillator Study. Am J Card 1999; 83: 68-73.

2. Connolly S, Hallstrom A, Cappato R, et al. Meta-Analysis of the Implantable Cardioverter Defibrillator Secondary Prevention Trials. Eur Heart J.2000; 21: 2071-78. 1.

3. The Antiarrhythmics versus Implantable Defibrillators (AVID) Investigators. A comparison of anti-arrhythmic drug therapy with implantable defibrillators in patients resuscitated from near-fatal ventricular arrhythmias. N Engl J Med 1997; 337: 1576-83.

4. Anderson JL, Hallstorm AP, Epstein AE, et al. The AVID Investigators. Design and results of the Antiarrhythmics vs Implantable Defibrillator (AVID) Registry. Circulation 1999; 99: 1692-1699.

5. Kuck K, Cappato R, Siebels J, Ruppel R. Randomized Comparison of Antiarrhythmic Drug Therapy with Implantable Defibrillators in Patients Resuscitated from Cardiac Arrest: the Cardiac Arrest Study of Hamburg (CAH). Circulation 2000; 102:748-54.

6. Wever E, Hauer R, Schrijvers G, et al. Cost-effectiveness of implantable defibrillator as first-choice therapy versus electrophysiologically guided, tired strategy in postinfarct sudden death survivors: a randomized study. Circulation 1996; 93: 489-496.

7. Connolly S, Gent M, Roberts RS, et al. Canadian Implantable Defibrillator Study (CIDS): A Randomized Trial of the Implantable Cardioverter Defibrillator Against Amiodarone. Circulation 2000; 101:1297-302 
8. Link $M$, Costeas $X$, Griffith $J$, et al. High incidence of appropriate implantable cardioverter-defibrillator therapy in patients with syncope of unknown etiology and inducible ventricular arrhythmias. J Am Coll Card 1997; 29: 370-375.

9. Moss A, Jackson Hall W, Cannon D, et al MADIT investigators. Improved survival with an implanted defibrillator in patients with coronary artery disease at high risk for ventricular arrhythmia. N Engl J Med 1996; 335: 1993-1940.

10. Gregoratos G, Cheitlin M, Conill A, et al. ACC/AHA Guidelines for Implantation of Cardiac Pacemakers and Arrhythmia Devices: A Report of the ACC/AHA Task Force on Practice Guidelines. Circulation 1998; 97: 1325-35. 
CHAPTER 3

\section{Dual Chamber Arrhythmia Detection in} the Implantable Cardioverter Defibrillator

Barbara Dijkman MD, Hein J.J. Wellens MD Department of Cardiology Academic Hospital Maastricht, Maastricht, The Netherlands

Published: Journal of Cardiovascular Electrophysiology, 2000;11:1105-1115. 


\section{ABSTRACT}

Background: Dual chamber ICD technology extended ICD therapy to more than termination of haemodynamically unstable ventricular tachyarrhythmias. It created the basis for dual chamber arrhythmia management in which dependable detection is important for treatment and prevention of both ventricular and atrial arrhythmias. Methods and results: Dual chamber detection algorithms were investigated in two Medtronic models of dual chamber ICD, the 7250 Jewel AF (33 patients) and the 7271 Gem DR (31 patients). Both ICDs use the same PR Logic algorithm to interpret tachycardia as ventricular (VT), supraventricular (SVT) or dual (VT+SVT). The accuracy of dual chamber detection was studied in $310 / 1367$ spontaneously occurring tachycardias in which rate criterion only was not sufficient for arrhythmia diagnosis. In 78 episodes there was a double tachycardia, in 223 episodes SVT was detected in the VT or VF zone, in 9 episodes arrhythmia was detected outside the boundaries of the PR Logic functioning. In $100 \%$ of double tachycardias the VT was correctly diagnosed and received priority in treatment. SVT was in $59(19 \%)$ episodes diagnosed as VT. The causes of inappropriate detection were: 1 ) algorithm failure - inability to fulfil the $\mathrm{PR}<\mathrm{RP}$ condition in atrial tachyarrhythmias with 1:1 AV conduction, and far field $R$ wave sensing intermittently present during sinus tachycardia, 2) programming settings - AF/AFL with ventricular rate above the SVT limit, 3) algorithm limitations atrial tachycardia with ventricular rate around the shortest programmable SVT limit and SVT redetection following VT therapy. Programming measures improved detection ability in $13 / 59$ of inappropriately detected arrhythmias.

Conclusions: Dual chamber detection algorithms evaluated in a subset of diagnostically difficult arrhythmias allow safe detection of double tachycardias but require further extension and programmability to improve VT:SVT discrimination rules. 


\section{INTRODUCTION}

Correct arrhythmia detection is a fundamental requirement for adequate ICD therapy. Since the first models of ICD many technological endeavors were made to improve device detection abilities. In the first devices the primary aim was to ensure safe recognition of life threatening ventricular tachyarrhythmias. Later attention was directed to discriminate fast (haemodynamically unstable) from slower (haemodynamically stable) ventricular tachycardias allowing the use of different therapeutic modalities (antitachycardia pacing, cardioversion, defibrillation). The next step was an attempt to differentiate between ventricular and supraventricular tachyarrhythmias to avoid inappropriate therapies. Technological tools that enabled these detection improvements included: programmability of tachycardia detection rate and duration, number of detection zones, detection enhancements such as sudden onset and rate stability, QRS width and morphology templates analysis. Presently available single chamber detection algorithms allow $90-100 \%$ sensitivity and $46-98 \%$ specificity of the ventricular tachyarrhythmia therapy (1-5).

One of the aims of dual chamber ICD technology was to further improve sensitivity and specificity of arrhythmia detection not only to withhold inappropriate therapy but also to allow dual chamber arrhythmia management. In this way the ICD is not only life saving $(6,7)$ because of ventricular arrhythmia treatment but it can also reduce morbidity of atrial arrhythmias.

The purpose of our study was to evaluate the functioning of currently used dual chamber detection algorithms in a subset of diagnostically difficult arrhythmias.

\section{METHODS}

\section{Dual chamber arrhythmia detection}

Dual chamber detection algorithms were investigated in two Medtronic models of dual chamber ICDs, the 7250 Jewel AF and the $7271 \mathrm{Gem}$ DR $(8,9)$. Both models are capable of detection and treatment of ventricular tachyarrhythmias, detection of supraventricular tachyarrhythmias, dual chamber pacing, mode switching and rate stabilization pacing. The Jewel AF has three modalities of atrial therapy: antitachycardia pacing, $50 \mathrm{~Hz}$ burst pacing and a cardioversion shock. Both models use the same "PR Logic" algorithm to interpret a tachycardia as ventricular (VT), supraventricular (SVT) or dual (VT+SVT). Arrhythmia classification is based on PR: $R P$ relation, $P P / R R$ regularity, evidence of ventriculoatrial (VA) conduction and far field $R$ wave sensing on the atrial channel (FFRS). 


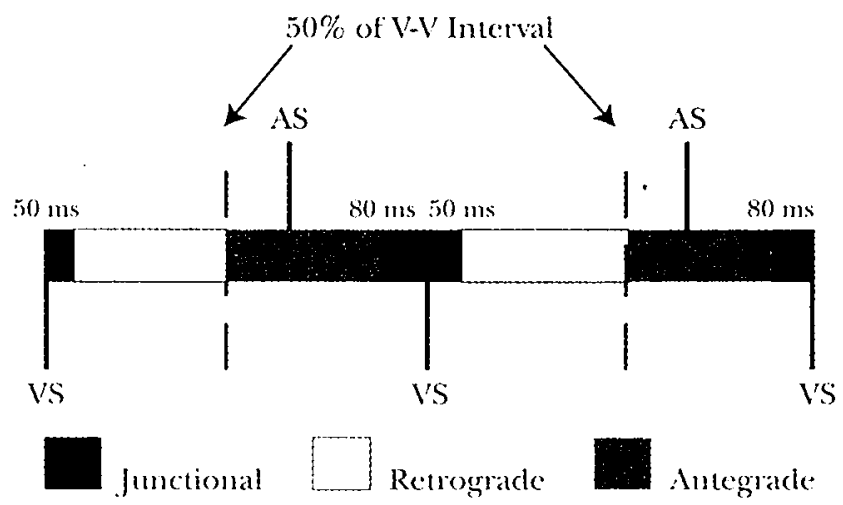

Figure 1. AV pattern recognition of PR Logic algorithm refers to the timing and the pattern of atrial events in relation to ventricular events. Each RR interval is divided in four zones to accommodate the expected atrial event during a tachyarrhythmia: antegrade $P(S T)$, retrograde $P(V T)$, junctional $P$ (in AV nodal tachycardia); AF waves can occur in various zones. AV relationship is evaluated in a number of consecutive ventricular beats.

PR Logic used in the Jewel AF and the Gem DR to discriminate between VT and SVT has both programmable and non-programmable elements. There are only two programmable dual chamber discrimination criteria: 1) type of $S V T$ for which VT:SVT rejection rules are applied: atrial fibrillation and atrial flutter (AF/AFL), sinus tachycardia (ST), and other 1:1 SVT; 2) the SVT limit: the ventricular cycle length until which ventricular therapy can be withhold in the presence of SVT. Most of the criteria used for the dual chamber arrhythmia discrimination are not programmable: 1) definition of SVT (AF/AFL criterion, ST criterion, other 1:1 SVT criterion); 2) ventricular cycle length regularity count; 3) $A: V$ dissociation count; 4) FFRS criterion. $A V$ pattern recognition, the key criterion of PR Logic, applied in all cases of dual chamber arrhythmia detection, is also not a programmable feature. In order to look at the relation and timing of the atrial and ventricular events each $R R$ interval is divided in four zones to accommodate an "expected" atrial event (fig.1).

The PR Logic algorithm was evaluated with respect to two diagnostic capabilities: 1) to ensure that ventricular detection is not disturbed by SVT criteria during a dual arrhythmia; 2) to prevent inappropriate ventricular labeling during a fast SVT that satisfies the ventricular rate criteria only.

\section{Patients}

In 64 patients (mean age $60 \pm 13$ years old) dual chamber Medtronic ICDs were implanted, the Jewel AF in 33 patients and the Gem DR in 31 patients. The ICD indi- 
cation was cardiac arrest with documented ventricular fibrillation (VF) in 30 patients, syncopal ventricular tachycardia (VT) in 28 patients and VT with other severe symptoms (pump failure, angina) in 6 patients. The etiology of the ventricular arrhythmia was coronary artery disease in 50 patients, dilated cardiomyopathy in 6 patients, other forms of cardiomyopathy in 6 patients and an apparent normal heart in 2 patients. Mean left ventricular ejection fraction was $31 \pm 13 \%$. In the Jewel AF group 29 patients had documented paroxysmal atrial tachyarrhythmia: 17 patients PAF, 2 patients PAFL, 9 patients both PAF and PAFL, 1 patient both paroxysmal atrial tachycardia (PAT) and atrioventricular nodal reentry tachycardia (AVNT). In the Gem DR group 1 patient had PAF and 1 patient AVNT.

Mean follow-up was $20 \pm 6$ months, with a total of 985 patient-months.

\section{ICD sensing function and detection programming}

The electrode hardware used during the implant procedure was selected depending on planned future high voltage ICD therapy (ventricular only or ventricular and atrial). The right ventricular sensing lead had true bipolar characteristics in 46 patients and integrated bipolar in 18 patients; mean $\mathrm{R}$ wave was $18(10-25) \mathrm{mV}$. A right atrial electrode with closely $(9.5 \mathrm{~mm})$ spaced tip-ring bipole was used for atrial sensing; mean $P$ wave being $3.6(2-8) \mathrm{mV}$. The right atrial lead was positioned in appendage in 4 patients, high right atrium anterior-lateral wall in 53 patients, low right atrium in 2 patients, the lateral wall 5 patients. There was no far field $R$ wave sensing of spontaneous and paced ventricular rhythms at implant. The initial sensing setting was programmed to $0.3 \mathrm{mV}$ for ventricle and atrium. In all patients the post pace atrial blanking period was programmed to $200 \mathrm{~ms}$, the duration of the not programmable blanking period after atrial sensed event was $100 \mathrm{~ms}$. Correct sensing was confirmed twice in-hospital shortly after implantation. Each patient underwent predischarge testing and at one month follow-up exercise stress testing. During predischarge testing a complete ventricular stimulation protocol as well as VF induction were performed via the ICD. In patients with paroxysmal atrial tachyarrhythmias induction of AF was done by fast burst pacing. During both investigations four surface ECG leads, intracardiac electrograms (Egm) and marker annotations $(M)$ were continuously registered.

Detection zones were programmed individually in each patient. Initial programming was based on information from arrhythmias occurring spontaneously and induced during electrophysiological study before implantation, operation and predischarge testing. Later during follow-up detection zones were adjusted when indicated using information about spontaneous recurrences. The value for the SVT discrimination limit was set to the nominal value of $320 \mathrm{~ms}$ in the first 12 patients and later adjusted, in all other patients it was programmed on an individual basis. 
Arrhythmia documentation

ICD documentation of spontaneously occurring atrial and ventricular tachyarrhythmias was retrieved from episode data and analyzed. Only arrhythmias with a complete registration of atrial and ventricular Egm, marker annotations and interval data were the subject of this study. Egm source programmed for data collection in Jewel AF was the wideband "Atip-Vring" stored 5 seconds from each tachyarrhythmia episode. Egm sources programmed in Gem DR, "A sense" and "V sense", were both stored during the whole arrhythmia episode.

\section{RESULTS}

\section{Arrhythmia recurrences}

During the follow-up period of 985 patient-months (mean 20 months (2-35) per patient) there were 1367 arrhythmia recurrences documented with Egm, marker annotation and interval data. In 1057 episodes the detected arrhythmia was a "single chamber" tachycardia: ventricular with VA dissociation $(n=457)$ or atrial with a ventricular rate outside the VT window $(n=600)$. In single chamber tachycardias the rate was sufficient for correct diagnosis. In the remaining 310 episodes tachycardia detection occurred both at the atrial and ventricular level. In these arrhythmias the VT:SVT discrimination rules were implemented before the tachycardia could be classified. The PR Logic algorithms were evaluated during these episodes.

\section{Safety of VT/VF detection}

The detection of true VT or VF was never interrupted or delayed by the rhythm detected on the atrial channel, neither in 457 "VT only" episodes nor in 78 double tachycardia (VT+SVT) episodes. The sensitivity to diagnose ventricular tachyarrhythmias within the programmed detection zones was $100 \%$ also in the presence of atrial arrhythmias.

\section{Arrhythmias detected with the dual chamber algorithm (Table 1)}

\section{1) Double tachycardias}

In 78/310 (25\%) episodes the PR Logic diagnosed dual arrhythmia (fig 2a). In the majority of episodes this was VT ( $\mathrm{n}=73$, mean CL $350 \mathrm{~ms}$ ), in 5 episodes VF. At the atrial level there was AF or AFL in 45/78 cases and AT in 20/78 cases. In addition there 
Table 1. VT + SVT Arrhythmias Detected with the Dual Chamber Algorithm

\begin{tabular}{|c|c|c|c|c|c|}
\hline Tachyarrhythmia & $\mathrm{Nb}$ cases & $\begin{array}{l}\text { Tachyarrhythmia } \\
\text { subcategories }\end{array}$ & & $\begin{array}{l}\mathrm{Nb} \text { of } \\
\text { patients }\end{array}$ & $\begin{array}{l}\text { Inappropriately } \\
\text { classified }\end{array}$ \\
\hline Double & 78 & $V T+A F$ & 40 & 7 & 0 \\
\hline \multirow[t]{3}{*}{ (atrial + ventricular) } & & $V T+A T$ & 20 & 4 & 0 \\
\hline & & $V T+"$ pseudo $\mathrm{AT}^{\prime \prime}$ & 13 & 6 & 0 \\
\hline & & $V T+A F$ & 5 & 2 & 0 \\
\hline \multirow[t]{3}{*}{ SVT in VT zone } & 223 & ST & 180 & 45 & 12 \\
\hline & & AT with $1: 1 \mathrm{AV}$ & 31 & 3 & 7 \\
\hline & & $A F, A F L$ & 12 & 3 & 7 \\
\hline \multicolumn{5}{|l|}{$\mathrm{AT}+$ ventricular rate above the } & 3 \\
\hline \multirow[t]{2}{*}{ Redetection SVT after VT therapy* } & 6 & ST & 4 & 2 & 4 \\
\hline & & AF & 2 & 1 & 2 \\
\hline Total & 310 & & $53^{* *}$ & 59 & \\
\hline
\end{tabular}

* Detection outside operation scope of PR Logic

${ }^{\star \star}$ In some patients there was more than one type of arrhythmia present

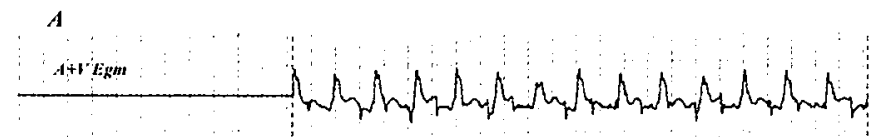

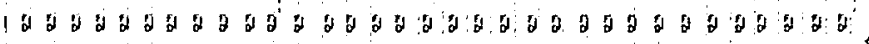
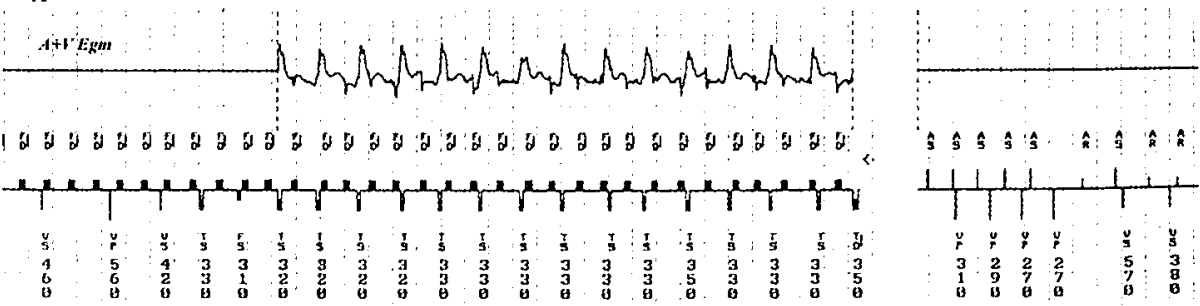

Marker

$B$

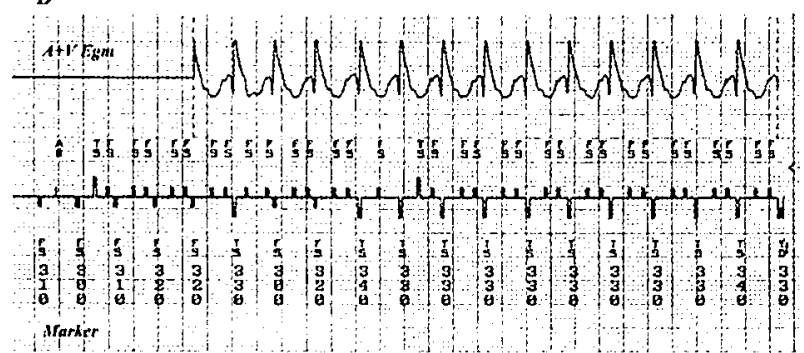

1 inV/mm 2stmms

Figure 2. Double tachycardia. A) Correct VT detection during AT. Following VT termination with pacing the AT continues. B) VT and "pseudo" AF caused by summation of far field R wave sensing and retrograde $P$ wave. Following VT termination with pacing, FFRS is still present during sinus rhythm. 

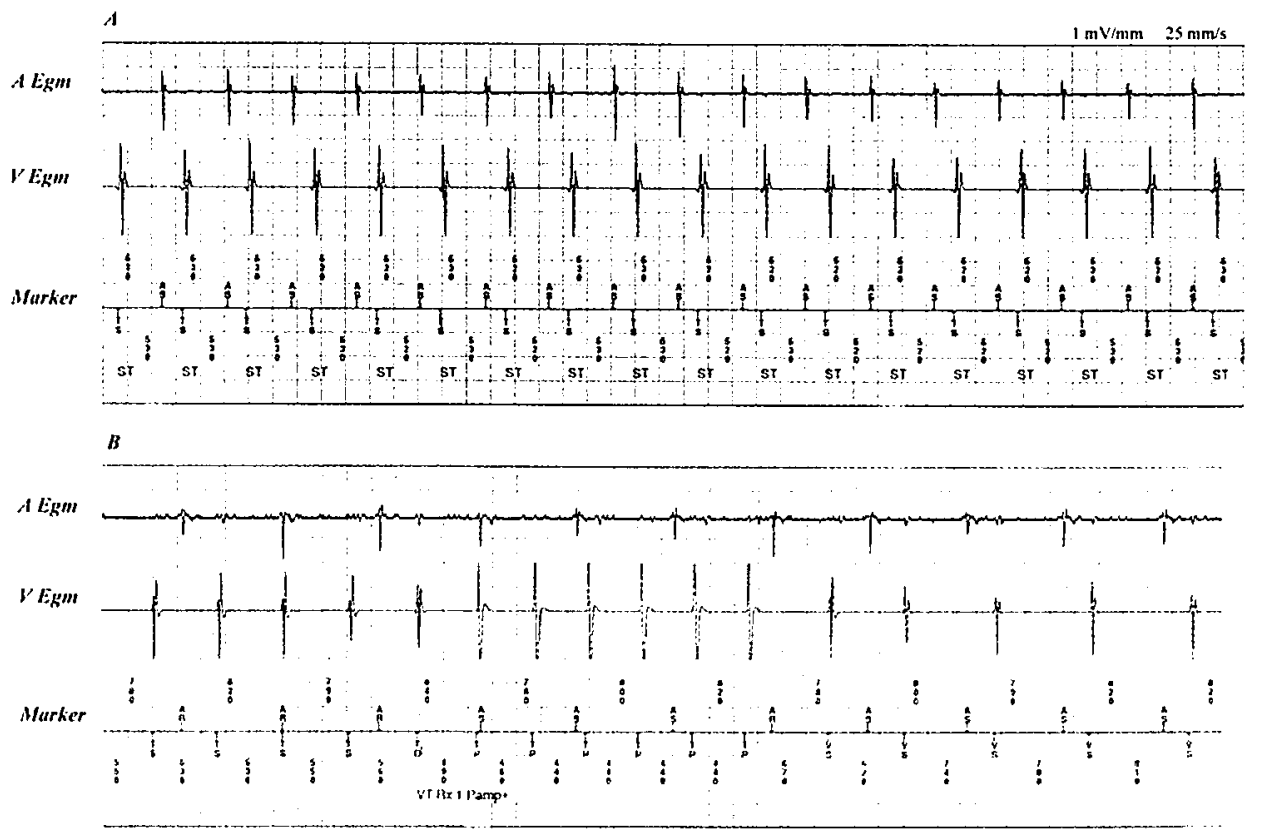

Figure 3. Dual chamber arrhythmia detection in a patient having rate overlap between VT and ST. A) Sinus tachycardia in the VT zone, the VT:SVT discrimination rules were applied correctly. B) Same patient: VT with a long cycle length.

were $13 / 78$ episodes in which during VT atrial arrhythmias were not really present but still detected and thus submitted to VT:SVT discrimination. These "pseudo" atrial tachyarrhythmias were due to the summation of retrograde $P$ waves and far field $R$ wave sensing (FFRS) on the atrial channel (fig 2b). In all double tachycardias the VT:SVT discrimination rules functioned appropriately regardless of the kind of atrial arrhythmia present during VT. Whenever VT was detected during atrial tachyarrhythmias the priority of treatment was given to VT. In one case the safety measure was applied by the ICD when VT detection appeared shortly after the delivery of atrial therapy; in that case the device considered that the VT might be the result of atrial therapy and disabled it.

\section{2) SVT in VT zone}

In $223 / 310(72 \%)$ of dual arrhythmia episodes the SVT was detected in the VT (sporadically VF) zone.

Simus tachycardia (ST) was the most frequently detected SVT in the VT zone $(180 / 223)$ having a mean CL of 550 (430-600) $\mathrm{ms}$ and occurring in 45 patients. The adequately diagnosed ST in the VT zone fulfilled the PR $<\mathrm{RP}$ criterion (fig 3 ). The majority of these detections were seen in patients in whom multiple VT morphologies were 


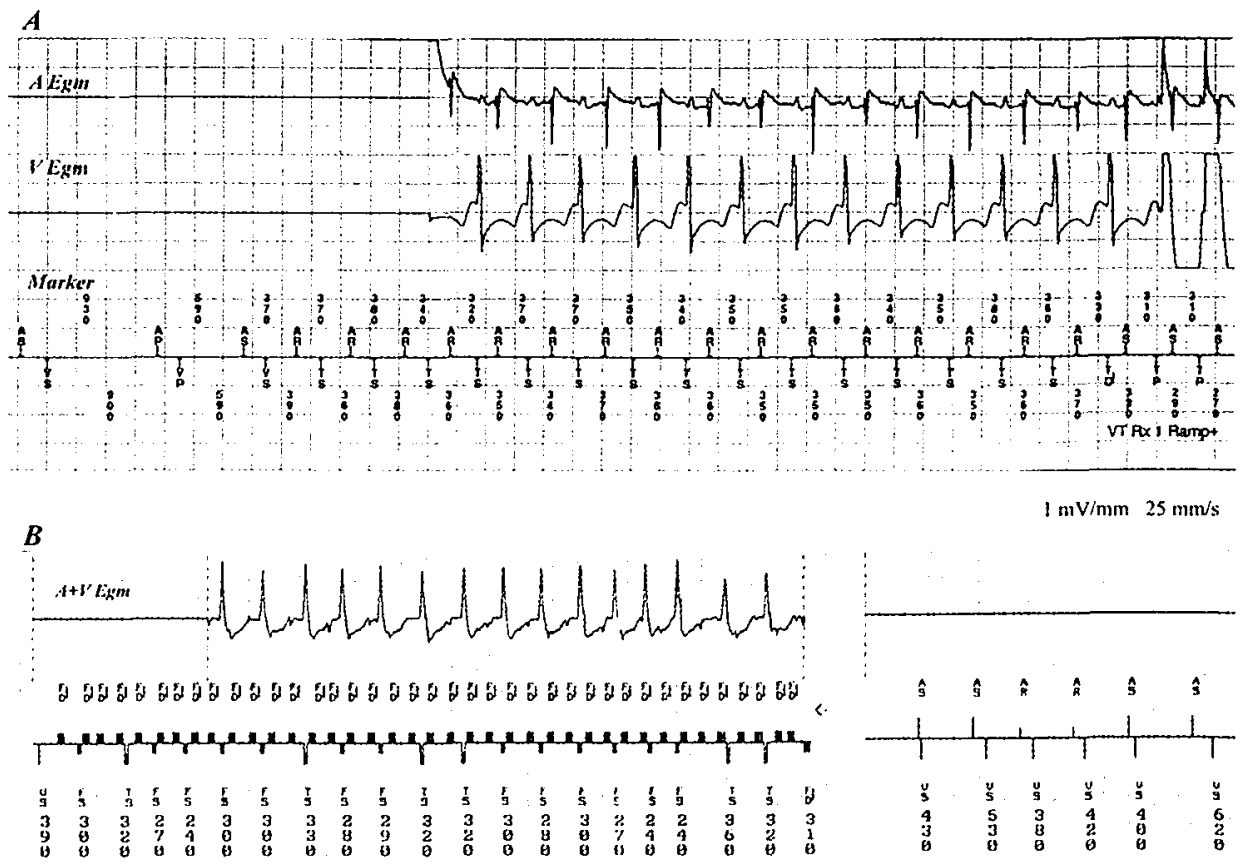

Figure 4. A) Atrial tachycardia with 1:1 AV conduction detected in the VT zone. There is no shortening of the PR interval at a higher heart rate. The antegrade $P$ falls in the zone of expected retrograde $P$. Failure of $P R$ Logic due to inability to fulfill $P R<R P$ criterion. B) Atrial fibrillation with fast ventricular rate detected as VF. Inappropriate detection occurs because the ventricular rate is above the programmed SVT limit.

present, some of the VTs being slow and having rate overlap with ST. With the exception of one episode, ST that were inappropriately detected as VT occurred during exercise testing in hospital, so no therapy followed.

Atrial tachycardia $(A T)$ with 1:1 AV conduction was the second most frequently $(31 / 223)$ occurring SVT detected in the VT zone and seen in 3 patients. Initiation of AT was always abrupt and the mean CL was 340 (310-380) ms. In all episodes of AT with 1:1 AV conduction the PR interval was longer than $R P$. The antegrade $P$ wave was detected in the retrograde zone causing inappropriate VT detection in all cases (fig 4a). All AT with 1:1 AV conduction detected in VT zone triggered VT therapy. In 28/31 cases it was antitachycardia pacing, which fortunately did not cause any proarrhythmia. In $3 / 31$ cases therapies proceeded till cardioversion, which terminated the AT.

Atrial fibrillation and atrial flutter $(A F / A F L)$ was documented in 12/223 episodes and resulted in 7 cases in ventricular rates causing detection above the programmed SVT 
limit (fig 4b). AF with fast ventricular rate detected as VT or VF occurred in 3 patients under extreme physical circumstances, like fever in a patient with severe pump failure, performance of an unusual amount of exercise in a short time, etc. In none of these patients AF/AFL with such a rapid ventricular rate was seen prior to implant. All 7 episodes of AF/AFL with ventricular rate above the SVT limit resulted in inappropriate cardioversion.

\section{3) Dual chamber detection outside the programmability of the PR Logic algorithm}

In 9/310 (3\%) episodes SVT was detected in VT/VF zone and the VT:SVT rejection rules were not applied. However it occurred in a setting that was an "expected behavior" of the detection algorithm.

Atrial Tachycardia in VF zone and around the shortest programmable SVT limit.

In 3/9 cases an AT with a mean CL of 250 (220-290) ms and 1:1 AV conduction resulted in detection in the VF zone (fig 5). Since detection was in the VF zone and some RR intervals above the shortest programmable SVT discrimination limit, dual chamber rejection rules were not applied any more. VF therapy was delivered, in one case resulting in the induction of AF and a second shock. The three cases of very rapid AT with 1:1 conduction occurred in 3 different patients under circumstances of anxiety, one in combination with exercise.

SVT redetection

In 6/9 VT episodes in 3 patients correct initial VT detection was followed by appropriate therapy resulting in VT termination but the initiation of SVT (4 ST, $2 \mathrm{AF}$ ). Since the VT:SVT rejection rules are available only during the initial detection, subsequent VT therapies (cardioversion) were delivered (fig 6).

\section{Causes of SVT being interpreted as VT/VF (table 2)}

In 59/310 (19\%) of the arrhythmia episodes detected using the rules of the dual chamber algorithm, tachycardia classification was not correct. In $43(73 \%)$ cases it was due to shortcomings of the VT:SVT discrimination criterion. In $7(12 \%)$ cases ventricular rate during SVT was faster than the programmed SVT discrimination limit. In the remaining $9(15 \%)$ cases the PR Logic was not applied as the arrhythmia classifier, which was in accordance with the normal function of the device.

1) $P R>R P$ relation was the most frequent $(63 \%)$ cause of SVT being inappropriately detected as VT. This diagnostic problem was especially obvious in atrial tachycardias with an abrupt onset and change of $C L$ when compared to the preceding sinus rhythm (fig 4a). Virtually all $31 \mathrm{AT}$ with 1:1 AV conduction detected in VT zone in our patients showed a $P R>R P$ relation and were erroneously detected as VT. Sinus tachycardias showed gradual onset and a longer mean $C L$. The $P R>R P$ relation was present in only $6 / 180$ ST (fig 7a). 
总

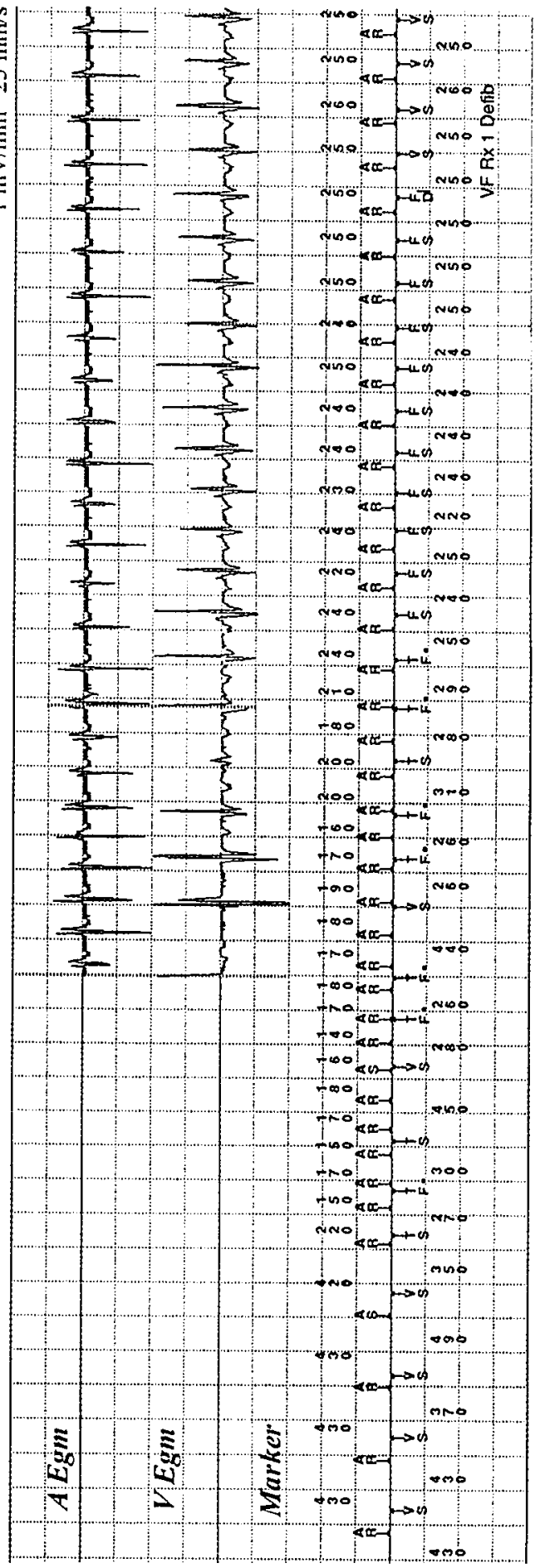


[98] Chapter 3
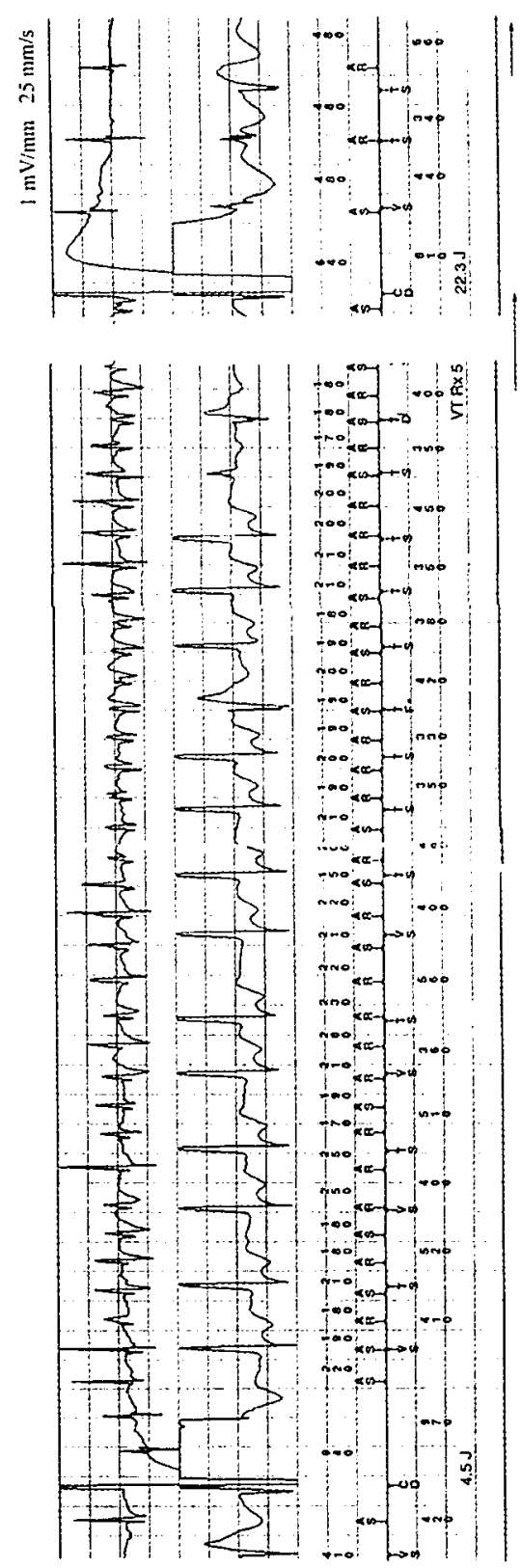

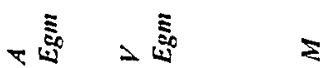

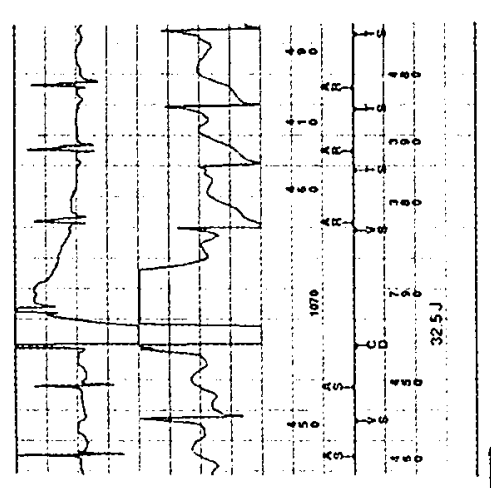

吾 导

至

点突

온

농

.

过岳

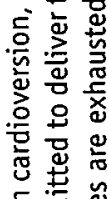

岳带

|3

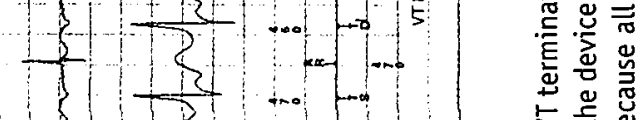

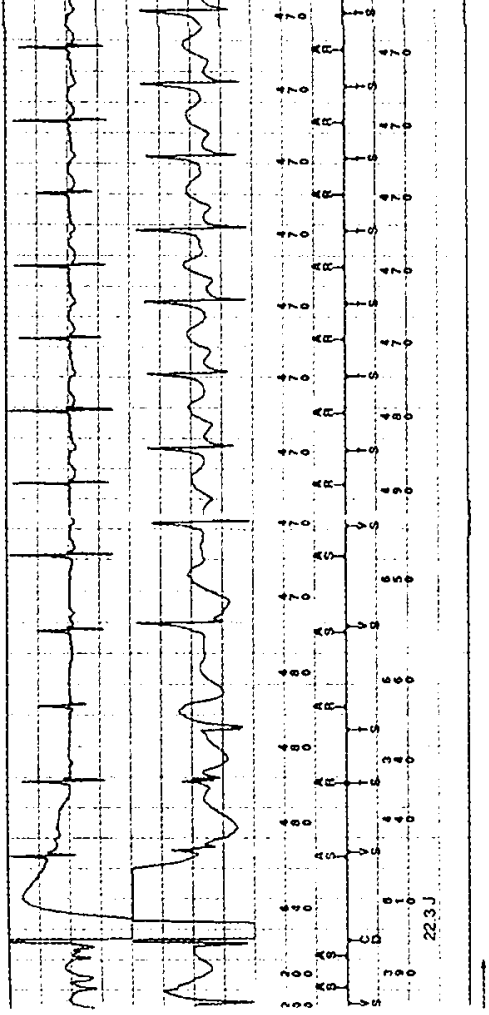

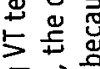

동 气

응 웅

요 홍 엉

递完

올

음 울을

놓 등

는군은

등 믕 흥

픈 흔

岳专

닌등드

등 突

总产 ผ

吉点焉

它它

至艺

온 는

它

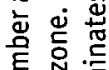

空

要

苛岳

。ํㅡㄴ

这产

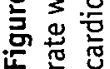


2) Far field $R$ wave sensing on the atrial channel (FFRS)

In $10 \%$ of misdiagnosed SVTs, ST was detected in the VT zone with FFRS being intermittently present. FFRS never fulfilled the $10 / 12$ criterion. It was not clear if that ever will be possible. In all cases already following 6-10 beats with evidence of FFRS, VT was detected (fig 8). FFRS had mean amplitude of 0.45 (range $0.15-1.2$ ) $\mathrm{mV}$ and was located 80 (range 0-200) ms after the ventricular sensed event. Evidences of FFRS as seen in atrial Egms were actually more frequently present than detected by the device; fortunately the amplitude of the FFRS signal was often to small to be recognized as an atrial sensed event.

3) SVT discrimination limit

In $12 \%$ of misclassified SVTs AF with a fast ventricular rate was present while the RR intervals were shorter than the programmed SVT discrimination limit. In those patients the reason for inappropriate detection were programming settings rather than failure of the algorithm.

4) Inappropriate detection but not PR Logic failure

Redetection of SVT in VT zone. Since the VT:SVT discrimination rules apply only to the initial detection, their absence on redetection cannot be seen as PR Logic failure but a limitation of detection principles in general. AT detected in the VF zone and around the shortest programmable $S V T$ Timit is another example of detection that is inappropriate but in which PR Logic functions as it was meant to do.

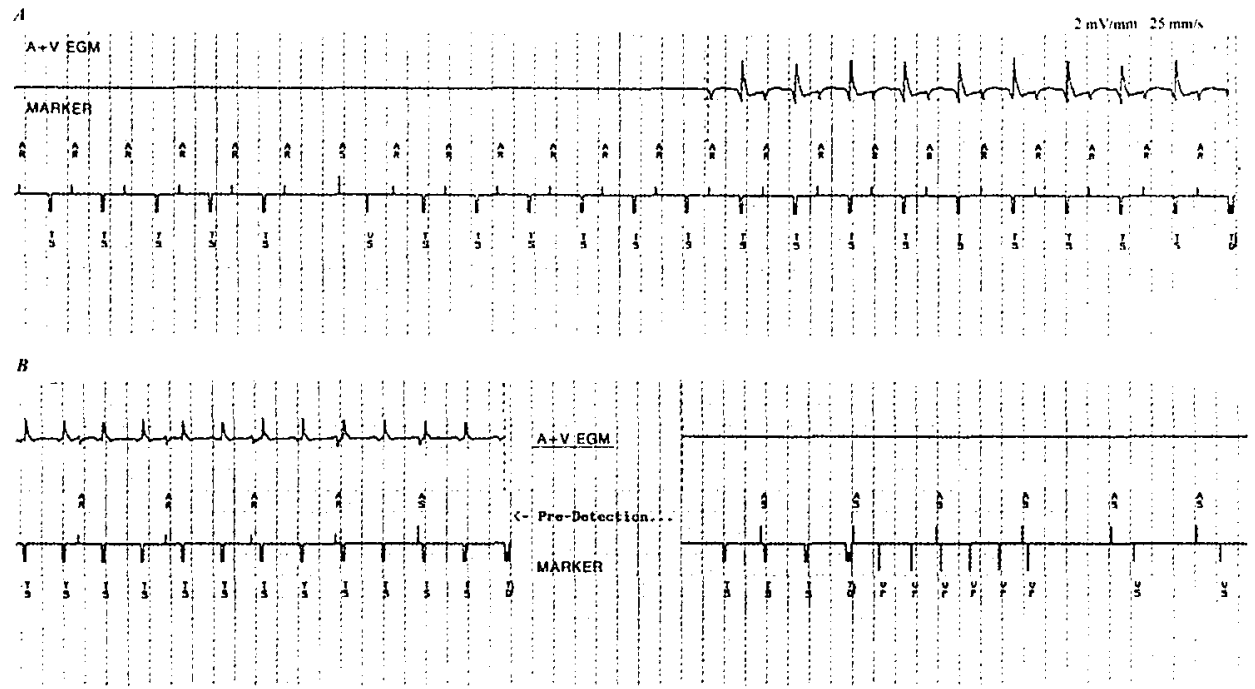

Figure 7. A). Inappropriate VT detection: sinus tachycardia with a long $P R$ interval resulting in $P R>R P$ ratio; one $P$ is not conducted at all. $B$ ). Same patient: during VT there is VA dissociation. Combining figures $7 A$ and $7 B$ in this patient, with poor antegrade and absent retrograde conduction, there is no use for a "reserve" zone for retrograde $P$. 


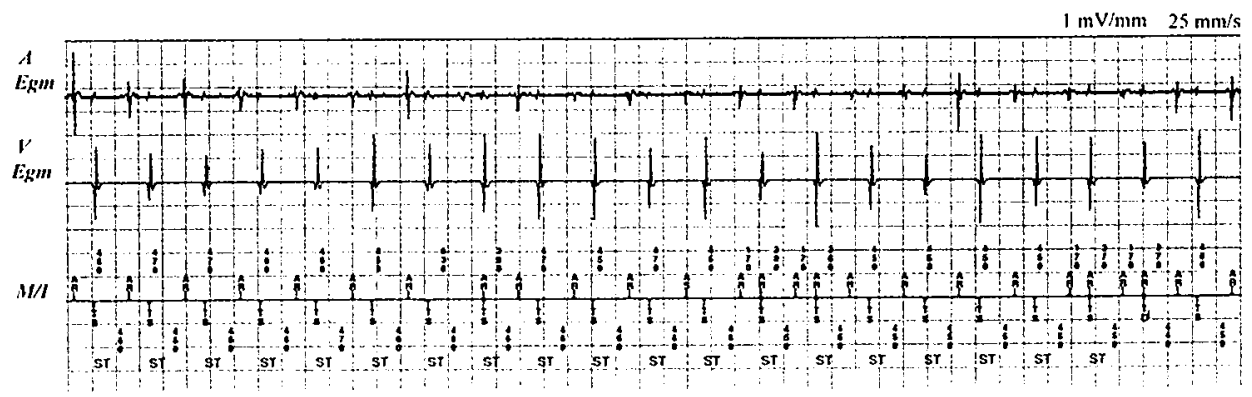

Figure 8. Sinus tachycardia in the VT zone. At the higher rate intermittent far field $R$ wave sensing occurs. Since the FFRS does not fulfill the 10/12 criterion, the tachycardia is interpreted as VT and not ST+FFRS. The FFRS deflection is present from the start of the registration, but only when the amplitude of the intrinsic $P$ decreases, FFRS is exposed.

Table 2. Types and Causes of SVT Interpreted as VT and Possibilities to Correct Diagnostic Mistakes in Present ICDs.

\begin{tabular}{llccc}
\hline SVT detected as VT & $\begin{array}{l}\text { Cause Mistaken } \\
\text { Detection }\end{array}$ & $\begin{array}{l}\text { Nb of } \\
\text { Patients }\end{array}$ & $\begin{array}{l}\text { Correction } \\
\text { possible }\end{array}$ \\
\hline ST in VT zone & PR>RP & 6 & 6 & 2 \\
& FFRS & 6 & 6 & 5 \\
AT 1:1 AVconduction & PR>RP & 31 & 3 & 0 \\
AF/AFL & SVT limit & 7 & 3 & 6 \\
AT in VF zone above SVT limit & beyond PR Logic & 3 & 3 & 0 \\
SVT onredetection & beyond PR Logic & 6 & 4 & 0 \\
Total & & 59 & 25 & 13 \\
\hline
\end{tabular}

\section{DISCUSSION}

Previous studies on arrhythmia detection capabilities in ICDs cannot always be compared because of differences in study design and algorithms tested (1-5). In our study not all detected tachycardias but only a subset of diagnostically difficult and spontaneously occurring arrhythmias were evaluated. There was also no rate limit set to consider a tachycardia for evaluation. Furthermore, we also analyzed arrhythmia episodes in which the PR Logic showed the "expected behavior" but the result was inappropriate therapy. Arrhythmia classification by ICD could be verified in the diag- 
nostics of episode data. This way not only device defined, but also the clinically relevant PR Logic performance was studied.

\section{Programming measures to improve detection specificity available in present ICDs and desirable in future devices}

\section{1) $P R: R P$ relation}

The AV recognition pattern of the PR Logic algorithm divides the $R R$ interval into four zones to accommodate the "expected" atrial events: antegrade and retrograde $P$ wave, FFRS, AF/AFL sensing (fig 1). This means that only atrial tachyarrhythmias, which are conform with that "expectation" can be appropriately detected. In ST or AT with 1:1 AV conduction and $P R>R P$, pattern recognition finds the antegrade $P$ at the place of the "expected" retrograde P wave. When this ST or AT is detected in VT zone, it is falsely interpreted as VT. Since the zones for antegrade and retrograde $\mathrm{P}$ are not programmable it is difficult to correct that classification error with the present algorithm.

In some cases the critical analysis of clinically documented ventricular and atrial tachyarrhythmias might help to program the VT detection zone "away" from SVT while maintaining a sufficient safety margin. However that measure means not challenging the algorithm rather than using it. Alternatively, programming the basic bradycardia-pacing mode to DDD with a short AV interval in order to restore $\mathrm{PR}<\mathrm{RP}$ seems acceptable only if a patient needs ventricular pacing and if that mode of pacing does not negatively affect the haemodymanic performance for those patients. The possible detrimental effects of $R V$ apical pacing in patients with decreased cardiac function makes chronic RV pacing to ensure a short $\mathrm{AV}$ interval an unacceptable choice to help the ICD properly detect arrhythmias.

Maladaptation of the PR interval to higher heart rates was often seen in our patients. During exercise induced ST 19\% patients showed absent or hardly measurable PR shortening. Telemetry monitoring before ICD implantation also showed failure of PR shortening during spontaneous rhythm acceleration in $25 \%$ patients. This means that potential SVT misdiagnosis due to PR $>$ RP could have happened more often than actually observed. Fortunately not every patient with prolonged $\mathrm{PR}$ interval during ST will at the same time have VTs with long CL. In a study of 108 patients, Paul et al (10) showed that in the absence of antiarrhythmic drugs, only $11 \%$ had sinus rates exceeding the rate of VT, but this proportion increased to $35 \%$ in patients on a single antiarrhythmic drug regimen and to $63 \%$ on a combination of antiarrhythmic drug therapy.

In patients with absent $V A$ conduction and a prolonged $P R$ interval during sinus and atrial tachycardia, it might be rational not to "reserve" a zone for the retrograde $\mathrm{P}$ in AV recognition pattern (fig 7). Only 4.4-28\% (11-13) of ventricular tachycardias are associated with 1:1 VA conduction. 
It would be useful if AV pattern analysis of the PR Logic were programmable with respect to a number of parameters: the (relative) duration of zones for antegrade versus retrograde $\mathrm{P}$ wave, the range of ventricular rates within which both zones are programmable, the duration of retrograde zone to be " 0 " in patients with absent VA conduction.

\section{2) Far field $R$ wave sensing on the atrial channel}

The FFR $S$ problem could be solved in $5 / 6$ patients by programming the atrial channel to less sensitive values. In the remaining patient such an adjustment was not possible there was not enough difference between the amplitude of FFRS and the P wave. It is essential during implant procedure to obtain $P$ wave amplitude that will allow the adjustment in $P$ sensitivity in the future. Programming the atrial channel less sensitive could cause intermittent atrial undersensing which in turn can also lead to inappropriate VT/VF detection (14). In patients in whom no atrial tachyarrhythmias need to be considered for treatment or mode switching there is still one less elegant way to bypass shortcomings of the FFRS criterion: programming the atrial channel paradoxically to a more sensitive setting. As a result FFRS is present on a beat-to-beat basis and at higher rates the short-long AA pattern is lost. Inappropriate "pseudo" atrial tachycardia is detected, but intermittent FFRS that results in ST detected as VT is no longer present and inappropriate VT therapy is withheld.

FFRS in atrium as a form of oversensing was encountered already in AAI and DDD pacing systems (15-17). Depending on sensing polarity, design and position of the atrial lead and signal filtering, it could be observed by some authors in up to $80 \%$ of patients. In dual chamber ICD with atrial arrhythmia management capabilities (mode switching, AF/AT therapy) atrial blanking is short in order to ensure a wide detection window for atrial events; short atrial blanking is a predisposing factor for FFRS.

FFRS showed to be a phenomenon of dynamic nature. Despite the fact that in none of our patients during the implant procedure FFRS was present, in $40 \%$ of the patients it could be provoked at least once on follow-up visits. Sometimes it was spontaneously present, sometimes only after provocation such as higher heart rate, sitting body position, following ventricular paced beats (contrary to sensed). The amplitude and the VA time of the FFRS showed variation within small limits from one measurement to another. At the nominal atrial sensing setting only in $2 / 28$ patients in whom FFRS was seen it was present on a beat-to-beat basis; in all other patients it was only intermittently present.

Not all circumstances of FFRS presence were of equal clinical relevance. For the dual chamber discrimination algorithm FFRS is important when present during a ventricular sensed rhythm occurring within the VT detection zone. Virtually each time during ST when FFRS appeared it led to inappropriate VT detection because the number or RR intervals with FFRS evidence required to confirm its presence never reached the $10 / 12$ criterion (fig 8 ). In patients in whom it is impossible to prevent 
FFRS by programming the atrial channel to less sensitive value other options are needed. It would be desirable if the $10 / 12$ criterion were programmable.

Continuous presence of FFRS leads to inappropriate detection of atrial arrhythmias. False detection of AF/AT might lead to erroneous pacing (18), inappropriate mode switching $(19,20)$ or atrial therapy. In these cases it does not matter if there is a ventricular sensed or a paced rhythm.

The solution for FFRS should come from ICD (and pacemaker) engineers: better filtering of atrial signals so that this phenomenon will not appear at all.

\section{3) SVT discrimination limit}

The SVT discrimination limit was programmed to the value that was well above the ventricular rate during SVTs documented prior to ICD implantation. However in 3 patients the ventricular rate during SVT crossed the SVT discrimination limit for a .period of time that was long enough to cause VT or VF detection. With growing confidence in dual arrhythmia detection and virtually no single example of VT underdetection during SVT, the SVT limit was later programmed in most patients to a shorter CL (i.e. $270-300 \mathrm{~ms}$ ). This measure solved in $6 / 7$ cases the problem of inappropriate AF detection as VT/VF.

In cases AF/AFL with fast ventricular rate exceeded the programmed SVT discrimination limit resulting in inappropriate VT detection the programming setting, more than an algorithm failure, was the cause of misdiagnosis. However reprogramming the SVT limit to shorter CL values is helpful only in some of the atrial arrhythmias previously misdiagnosed due to SVT limit. Potentially useful discrimination rules such as PR:RP ratio are less reliable when the heart rate is very high. Also the beat-to-beat variability in $R R$ interval during $A F$ tends to decrease with increasing rates (21). At short CL the ventricular regularity count of $<50 \%$ required to recognize AF in the VT zone might not be fulfilled.

\section{4) Inappropriate detection but not $P R$ Logic failure}

\section{AT with 1:1 AV conduction in the VF zone around the SVT limit}

Rapid AT with 1:1 AV conduction still remains a diagnostic problem for the dual chamber detection algorithm. The SVT discrimination limit which is programmable as low as $240 \mathrm{~ms}$ is already liberal and with few exceptions sufficient to allow almost all episodes of AF/AT to be recognized as SVT. In view of the questionable reliability of the discrimination algorithm at short tachycardia $\mathrm{CL}$ and the potential consequences of "diagnostic mistakes", setting the SVT discrimination limit at 240 ms seems justified. More observational data and studies are necessary before we can negotiate with that limit. 


\section{Redetection}

The possibility of dual chamber redetection should be present, since delivering inappropriate therapy of increasing aggressiveness is not safe. Sequences of ICD therapies when an appropriate shock for VT/VF induces AF, for which another shock is delivered (because there is no VT:SVT discrimination on redetection), causing again VF, etc. may end up with VT or VF for which no further therapy is available (22).

\section{Other measures to improve arrhythmia diagnosis-detection enhancements and extension of diagnostics}

In "difficult" arrhythmias that remain a diagnostic problem for PR Logic, it might be useful to add some of the single chamber detection enhancements, like QRS morphology analysis.

The concept of monitoring some of the hardware parameters, already present in the Gem DR, could be extended to additional measurements. "Automeasurements" might be helpful in selecting parameters to optimize programming of detection algorithms. For instance keeping record of the $P$ wave amplitude during atrial arrhythmias and during sinus rhythm might help to optimize the atrial sensing in patients with FFRS. Recording QRS templates during sinus rhythm might help to implement an additional detection enhancement. Measurement of VA intervals in conducted ventricular beats can give some idea about FFRS.

Growing complexity of detection algorithms makes correct arrhythmia diagnosis dependable on more than one parameter (23). Extensive documentation, with dual chamber Egm, markers and intervals is necessary from before the arrhythmia onset through all the therapeutic steps until the arrhythmia is declared terminated.

\section{LIMITATIONS OF THE STUDY}

Since Gem DR provides more complete arrhythmia documentation than the Jewel AF, the possibilities to follow the detection and redetection process are not the same in both devices.

Some of the presented data were obtainable only in Gem DR, for instance episodes of sinus tachycardias occurring in VTs zone with correctly withheld therapy. In supraventricular arrhythmias the Jewel AF provides Egm documentation of treated episodes only. Furthermore, type and timing of sensed events could better be judged in separate A Egm and V Egm in Gem DR than in the wideband A+V Egm in Jewel AF. 


\section{CONCLUSIONS}

Dual chamber detection algorithms allow safe detection of all double tachycardias and correct diagnosis in $81 \%$ of supraventricular tachycardias occurring in the VT zone. The causes of arrhythmia misinterpretation were: the PR Logic failures (73\%), the PR Logic limitations (15\%) and the programming settings (12\%). Extension and programmability of the algorithms is required to improve detection accuracy in this subset of diagnostically difficult arrhythmias.

\section{REFERENCES}

1. Swerdlow CD, Chen PS, Kass RM, Allard JR, Peter CT. Discrimination of ventricular tachycardia from sinus tachycardia and atrial fibrillation in tired-therapy cardioverter-defibrillator. $\mathrm{J}$ Am Coll Card 1994; 23: 1342-1355.

2. Swerdlow CD, Ahern T, Chen PS, Hwang C, Gang E, Mandel W, Kass RM, Peter CT. Underdetection of ventricular tachyarrhythmia by algorithms to enhance specificity in a tired-therapy cardioverter-defibrillator. J Am Coll Card 1994; 24: 416-424.

3. Neutzner J, Pitschner H, Schlepper M. Programmable VT detection enhancements in implantable defibrillator therapy. PACE 1995; 18: 539-547.

4. Brachmann J. Swerdlow CD, Mitchell BL, Miller T, van Veen BK for the Worldwide 7218 ICD investigators. Worldwide experience with the electrogram width feature for improved detection in an implantable pacer-cardioverter-defibrillator. J Am Coll Card 1997; 28:115A.

5. Brugada J, Mont L, Figueiredo M, Valentino M, Matas M, Navarro-Lopez F. Enhanced detection criteria in implantable defibrillators. J Cardiovasc Electrophysiol 1998; 9: 261-268.

6. The Antiarrhythmics versus Implantable Defibrillators (AVID) Investigators. A comparison of anti-arrhythmic drug therapy with implantable defibrillators in patients resuscitated from near-fatal ventricular arrhythmias. N Engl J Med 1997; 337: 1576-83.

7. Anderson JL, Hallstorm AP, Epstein AE, Pinski SL, Rosenberg Y, Nora MO, Chilson D, Cannom DS, Moore R. The AVID Investigators. Design and results of the Antiarrhythmics vs Implantable Defibrillator (AVID) Registry. Circulation 1999; 99: 1692-1699.

8. Jewel AF Arrhythmia Management Device system reference guide. Medtronic, Inc. 1996.

9. Gem DR dual chamber implantable cardioverter defibrillator system reference guide. Medtronic, Inc. 1997.

10. Paul V, Bashir $Y$, Anderson M, Ward D, Camm J. Antitachycardia pacing and antiarrhythmics combined: a recipe for misdiagnosis. PACE 1991; 14: 722 (A).

11. Wellens $\mathrm{H}, \mathrm{Bar} F$, Lie $\mathrm{K}$. The value of the electrocardiogram in the differential diagnosis of a tachycardia with a widened QRS complex. Am J Med 1978; 64:27-33.

12. Militianu A, Salacata A, Meissner M, Grill C, Mahmud R, Palti AJ, Ben David J, Mosteller R, Lessmeier, Baga JJ, Pires LA, Schurger CD, Steinman RT, Lehman MH. Ventricular conduction capability and prevalence of $1: 1$ retrograde conduction during inducible sustained monomorphic ventricular tachycardia in 305 implantable cardioverter defibrillator recipients. PACE 1997; 20: 2378-2384. 
13. Li H, Thakur R, Yee R, Klein G. Ventriculoatrial conduction in patients with implantable cardioverter defibrillators: implications for tachycardia discrimination by dual chamber sensing: A reassessment. PACE 1997:20;916-922.

14. Glotzer TV, Radoslovich GA, Neglia J, Zimmerman JM. Variability of atrial sensing during exercise in a patient with a dual chamber ICD caused inappropriate ICD discharges. PACE 1999; 22:1838-1841.

15. Brouwer J, Nagelkerke D, Heijer den P, RuiterJH, Mulder H, Begemann MJ, Lie KI. Analysis of atrial sensed far-field ventricular signals: A reassessment. PACE 1997: 20: 916-922.

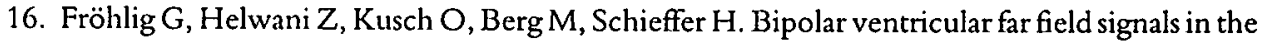
atrium. PACE 1999; 22: 1604-1613.

17. Schüller $\mathrm{H}, \mathrm{R}$ euter J, Clausson $\mathrm{E}$, Markowitz $\mathrm{T}$. Far field $\mathrm{R}$ wave sensing-an old problem reappearing. PACE 1996; 19: 631 (abstract).

18. BrickerJT, Garson AJr, Traweek MS, Smith RT, Ward KA, Vargo TA, Gilette PC. The use of exercise testing in children to evaluate abnormal pacemaker function not apparent at rest. PACE 1985; 8: 656-660.

19. Fröling G, Kindermann M, Heisel A, Lawall P, Berg M, Schwaab B, Shieffer H. Mode switch without atrial tachyarrhythmia. PACE 1996; 19: 592 (abstract).

20. Ellenbogen KA, Mond HG, Wood MA, Barold SS. Failure of automatic mode switching: recognition and management. PACE 1997; 20: 268-275.

21. Kuelkamp V, Mewis Ch, Suchalla R, Bosch R, Doernberger V, Seipel L. Rate dependence of RR stability during atrial fibrillation and ventricular tachyarrhythmias. Circulation 1998:98; 713 (A).

22. Birgersdotter-Green U, Rosenqvist $M$, Lindemans FW, Ryden L, Radegran K. Holter documented sudden death in a patient with an implanted defibrillator. PACE 1992: 15: 1008-1014

23. Sarter BH, Callins DJ, Gottlieb GD, Schwartzmann DS, Marchlinski FE. Implantable defibrillator diagnostic storage capabilities: evolution, current status, and future utilization. PACE 1998; 21: 1287-1298. 


\section{CHAPTER 4}

\section{Importance of the Atrial Channel for Ventricular Arrhythmia Therapy in the Dual Chamber Implantable Cardioverter Defibrillator}

Barbara Dijkman MD, Hein J.J. Wellens MD Department of Cardiology

Academic Hospital Maastricht, Maastricht, The Netherlands

Published: Journal of Cardiovascular Electrophysiology 2000;11:1309-1319. 


\section{ABSTRACT}

Background: Performance of dual chamber ICD systems has been judged based on functioning of the VT:SVT discrimination criteria and DDD pacing. The purpose of this study was to evaluate the use of the dual chamber diagnostics to improve the electrical and antiarrhythmic therapy of ventricular arrhythmias.

Methods and results: Information about the atrial and ventricular rhythm in relation to ventricular arrhythmia occurrence and therapy was evaluated in 724 spontaneous arrhythmia episodes detected and treated by three types of dual chamber ICDs in 41 patients with structural heart disease. Device programming was based on clinically documented and induced ventricular arrhythmias. In ambulatory patients sinus tachycardia preceded ventricular arrhythmias more often than in hospital during exercise testing and the incidence of these VTs could in only 2 patients be reduced by an increase in dose of beta-blocking agent. In 5 patients in whom sinus tachycardia developed after onset of haemodynamic stable VT, propranolol was more effective, as far as incidence of VT and pace-termination was concerned, than class III antiarrhythmics combined with an other beta-blocking agent. Atrial arrhythmias were in all but 3 cases present a longer time before the onset of ventricular arrhythmias. During atrial arrhythmias fast ventricular rates before the onset of ventricular rate were observed more often than RR irregularities and short-long RR sequences. Dual chamber diagnostics allowed proper interpretation of detection and therapy outcome in patients with different types of ventricular arrhythmia.

Conclusions: The advantages of dual chamber ICD system go further than avoiding the shortcomings of the single chamber system. Information from the atrial chamber allows better device programming and individualization of drug therapy for ventricular arrhythmia. 


\section{INTRODUCTION}

Technology of the dual chamber implantable cardioverter defibrillator (ICD) was driven by two requirements. First, the need to have DDD pacing possibilities integrated in an ICD system. Second, improved detection specificity to avoid inappropriate therapies for supraventricular arrhythmias. The performance of the dual chamber ICDs has so far been judged based on the performance of these two features (1). However it is still the ventricular arrhythmia, which is the primary aim of ICD therapy, and it is not entirely clear if dual chamber algorithms could positively influence device therapy other than to help withholding false shocks. The purpose of this study was therefore to evaluate the use of information from the atrial channel to guide antiarrhythmic and electrical therapy of ventricular arrhythmias.

\section{METHODS}

\section{Patients and their arrhythmias}

The impact of the relation between the atrial and ventricular rhythm on arrhythmia therapy was evaluated in ventricular arrhythmias occurring spontaneously in 41 patients ( $57 \pm 15$ years old) after dual chamber ICD implantation.

The ICD indication was cardiac arrest with documented ventricular fibrillation (VF) in 24 patients, syncopal ventricular tachycardia (VT) in 9 patients, VT with pump failure in 3 patients and recurrent VT after repeated radiofrequency ablations in 5 patients. Eleven patients had paroxysmal atrial fibrillation (PAF). The arrhythmia etiology was coronary artery disease in 26 patients, dilated cardiomyopathy in 10 patients, valvular disease in 2 patients, arrhythmogenic right ventricular dysplasia in 2 patients and sarcoid heart disease in 1 patient. The mean left ventricular ejection fraction was $33 \pm 11 \%$. Antiarrhythmic drugs that were used at the time of implant and follow-up were amiodarone ( $200 \mathrm{mg} /$ daily) in 20 patients and sotalol $(160 \mathrm{mg} / \mathrm{daily})$ in 7 patients. A beta blocking agent was used in 37 patients, in 30 patients long acting metoprolol 100 (range 50-150) $\mathrm{mg} /$ daily and in 7 patients carvedilol 25 (range 12.5-50) $\mathrm{mg} /$ daily. The doses of the beta-blocking agent initially prescribed were titrated to achieve specific therapeutic effects in the individual patient, for example control of anginal complaints or blood pressure; rate control during PAF or treatment of exercise induced VT. The dose was diminished in case of intolerability or temporarily diminished during worsening of pump failure.

Each patient underwent predischarge testing during which VF induction and programmed electrical stimulation were performed. One month after implantation each patient underwent exercise testing. The initial programming of the ICD detec- 
tion zones and therapies was based on clinical documentation of the ventricular arrhythmia, findings during electrophysiological study before and after implantation and relevant data from exercise testing.

\section{Arrhythmia detection and documentation by ICD}

Three types of dual chamber ICDs of two manufacturers were implanted, the Ventak AV (I, II, III) models of Guidant (20 patients), the $7271 \mathrm{Gem} \mathrm{DR} \mathrm{(10} \mathrm{patients)} \mathrm{and} \mathrm{the}$ 7250 Jewel AF (11 patients) models of Medtronic.

The ventricular arrhythmia detection in the dual chamber Guidant ICDs is based on rate and duration criteria and in Medtronic ICDs on rate and number of intervals. The detection zones for VT, fast VT and VF in ICDs of both manufacturers are not the same. There are three separate zones in the Guidant ICDs, only two zones in the Jewel $\mathrm{AF}$ and two zones with an overlapping third one, which can be coupled to VT or VF zone in the Gem DR. Since device-based arrhythmia diagnosis may differ due to different zones definition in the three ICDs and due to individual programming, the cycle length (CL) value was used as ventricular arrhythmia classifier in this study. We considered a ventricular arrhythmia to be VT if its CL was 300-600 msec and fast VT if the CL was $<300 \mathrm{msec}$.

During AF the ventricular rate was considered fast if it exceeded 100 beats $/ \mathrm{min}$. A sinus rate above 100 beats/min was defined as sinus tachycardia.

The relation between atrial and ventricular rhythm from the onset until the termination of spontaneously occurring ventricular arrhythmia was evaluated using the dual chamber ICD diagnostics. Only ventricular arrhythmias with atrial and ventricular electrograms (A Egm, $V$ Egm), marker annotation (M) and interval data were the object of this study. The Ventak AV models and the Gem DR provide A Egm and V Egm registration from the onset of the arrhythmia until its termination. The Ventak AV II and III models provide in addition to rate A Egm and V Egm also the ventricular shock Egm (Sh Egm). The Jewel AF provides the combined wideband A+V Egm registration of the initial arrhythmia detection and marker channel registration of arrhythmia termination.

\section{RESULTS}

During the follow-up period of $30 \pm 10(2-48)$ months 724 recurrent ventricular arrhythmias with complete Egm and marker registration were documented. There were 47 fast VTs ( 8 were VF) and 677 VTs. The fast VT had a mean CL of 275 (130-290) msec, were syncopal in 21 cases, occurred during sleep in 3 cases. The VTs had a mean CL of 355 (300-600) msec and were haemodynamically well tolerated 
within the timeframe of their duration. The atrial rhythm could be evaluated at different stages of a ventricular arrhythmia episode.

\section{Sinus tachycardia preceding the onset of ventricular arrhythmia}

On exercise testing after ICD implantation sustained VTs preceded by sinus tachycardia occurred in 4 patients. In these patients the dose of their beta-blocking agent was increased 1.5-2 times and the test repeated. During second testing sustained VT was no longer induced in 2 patients, but still initiated in 2 other patients. In the last 2 patients further increase of beta-blocker was not tolerated and exercise testing was not repeated. Therefore 39 patients entered the follow-up period without exercise induced ventricular arrhythmia.

In spontaneously occurring ventricular episodes dual chamber Egm showed sinus tachycardia preceding the onset of 60/724 (8\%) ventricular arrhythmia in 10 patients. The ensuing arrhythmia was fast VT in 2 and VT in 58 cases.

In 10 patients in whom sinus tachycardia preceded VT onset, the initial dose of the beta-blocking agent was increased 1.5-2 times in 2-3 steps. This resulted in reduction of VT recurrences by $>50 \%$ in only $2 / 10$ patients.

In two young patients with arrhythmogenic right ventricular dysplasia the dual chamber Egm added valuable information about their ventricular arrhythmia. Before ICD implantation VF was clinically documented in one patient and a fast VT with CL $240 \mathrm{msec}$ in the other patient. Both patients while on sotalol and metoprolol, were inducible only for fast VTs and showed no VTs during (maximal) exercise testing; the last test being performed twice in each patient. The dual chamber Egm documentation of spontaneous arrhythmia episodes in these patients showed that $5 / 10$ recurrent VTs were preceded by sinus tachycardia of a lower rate than the sinus tachycardia on exercise testing in hospital. The VT CL was in $8 / 10$ recurrent VTs considerably $(>120$ msec) longer than documented before implantation. The transition between sinus tachycardia and VT was accompanied by only a 30-50 msec change in CL (Fig 1). Information provided by both $\mathrm{A}$ Egm and V Egm was helpful to detect the slower VTs and adjust programming parameters. Both patients had no recollection of any physical activity of the same magnitude as during in-hospital exercise testing.

\section{Atrial and ventricular arrhythmias}

Atrial tachyarrhythmias were present at the time of a ventricular arrhythmia in 52/724 (7\%) documented ventricular arrhythmia episodes. However in a subgroup of 11 patients known with atrial arrhythmias before ICD implantation, 52/252 (21\%) VT episodes were dual arrhythmias. The A Egm registrations showed atrial tachycardia in 

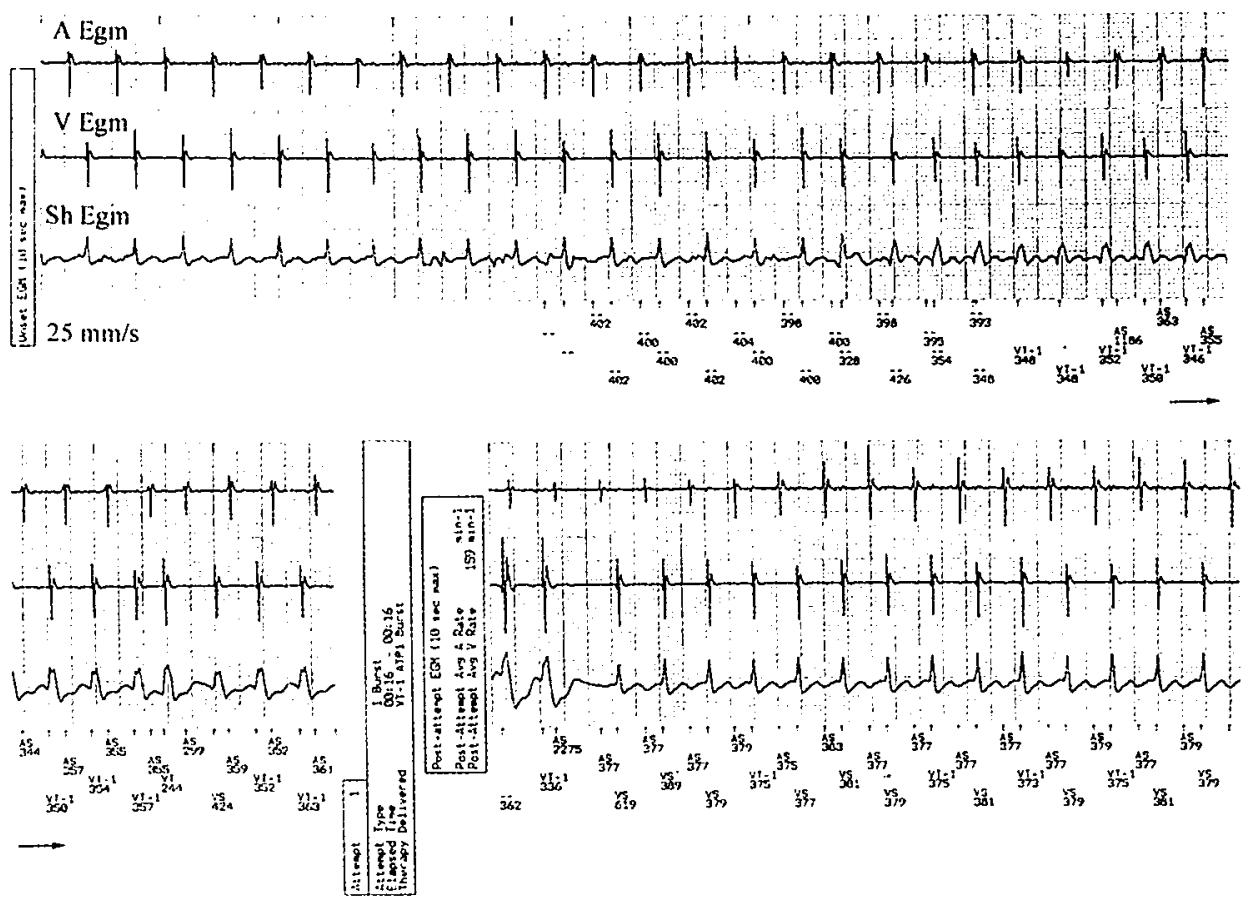

Figure 1. A) Sinus tachycardia before the onset of VT with only a $50 \mathrm{msec} C L$ difference between the two tachycardias. After 6 beats with VA dissociation there is 1:1 VA conduction. Sinus tachycardia continues following pace-termination of VT. Differences in intracardiac V Egm during sinus tachycardia and VT are not apparent but the Shock Egm points at VT initiation and termination. In the presence of 1:1 retrograde conduction sinus tachycardia developing after the onset of VT cannot be evaluated.

28 cases and AF in 24 cases. Since the total number of documented atrial arrhythmias was 592 , only $0.9 \%$ of them became dual tachycardia, atrial and ventricular.

Only in 3 episodes the onset of atrial tachycardia was immediately followed by the onset of VT (fig 2). In all other cases atrial arrhythmias were present for a longer time, hours to days before ventricular arrhythmias occurred. Rapid AV conduction during atrial arrhythmias resulted in ventricular rates of more than 100 beats $/ \mathrm{min}$ before VT initiation in 30 (58\%) cases. Changing AV conduction resulting in variation in $R R$ intervals at the ventricular level was observed before VT onset in $5(10 \%)$ cases. In the remaining cases the ventricular rate was neither fast nor irregular (fig 3).

In 2 patients $A F$ started during VT. In one during fast VT with 1:1 VA conduction, in the other patient there was VA dissociation (fig 4).

Atrial arrhythmias induced by defibrillation and cardioversion shocks were seen in $9 / 131(7 \%)$ cases of high voltage ventricular therapy. The single chamber V Egm and 

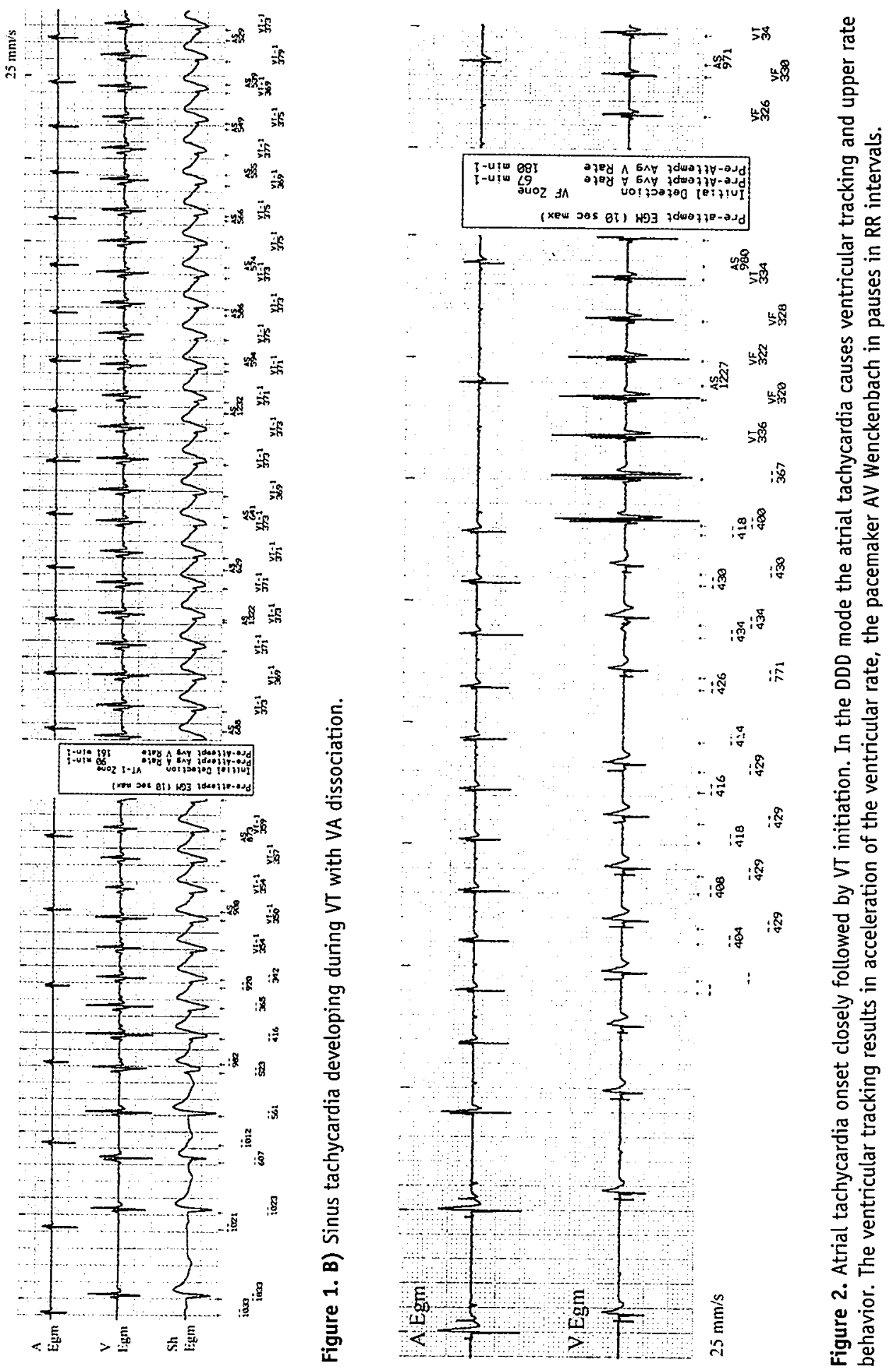
A

$25 \mathrm{~mm} / \mathrm{s}$
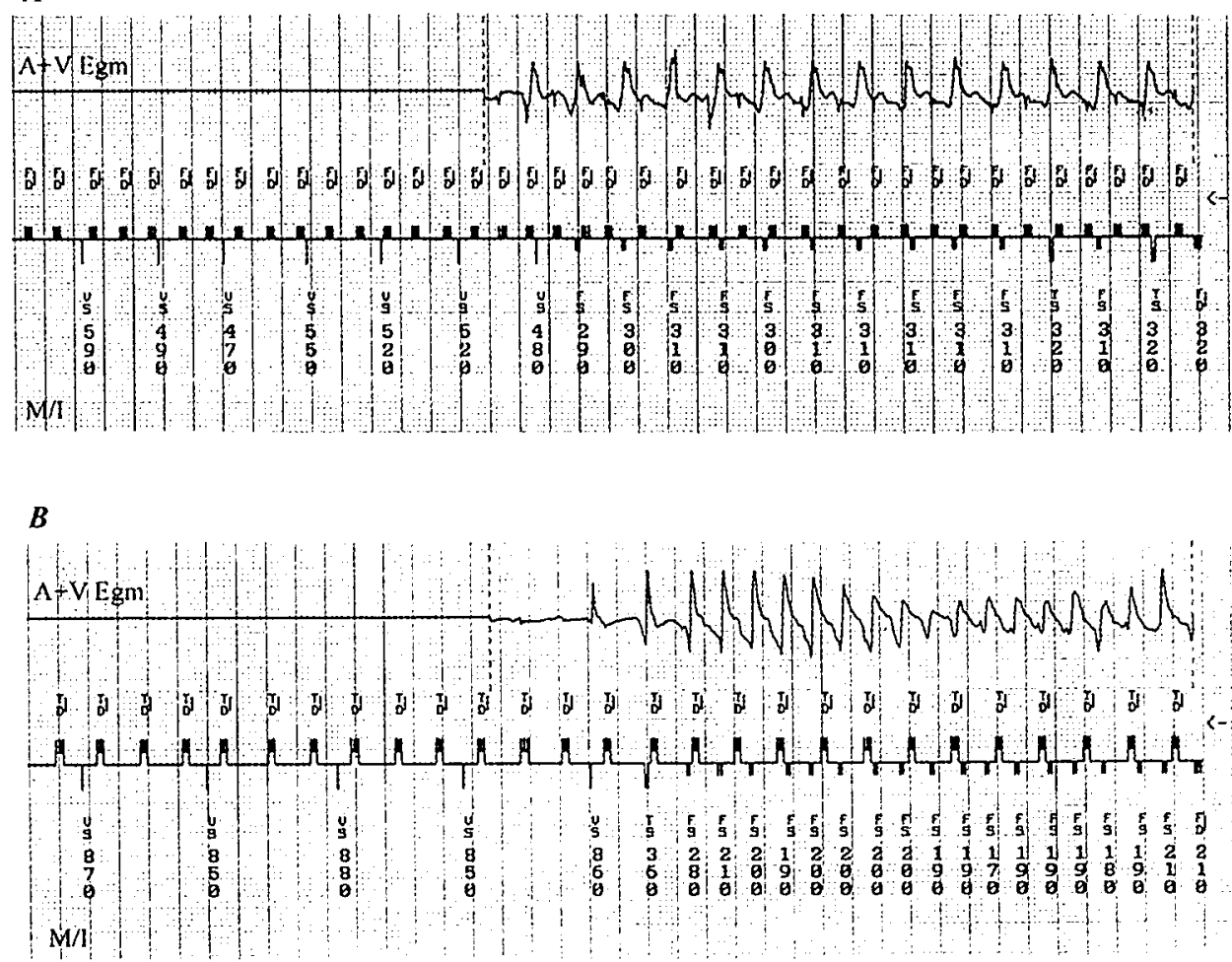

C

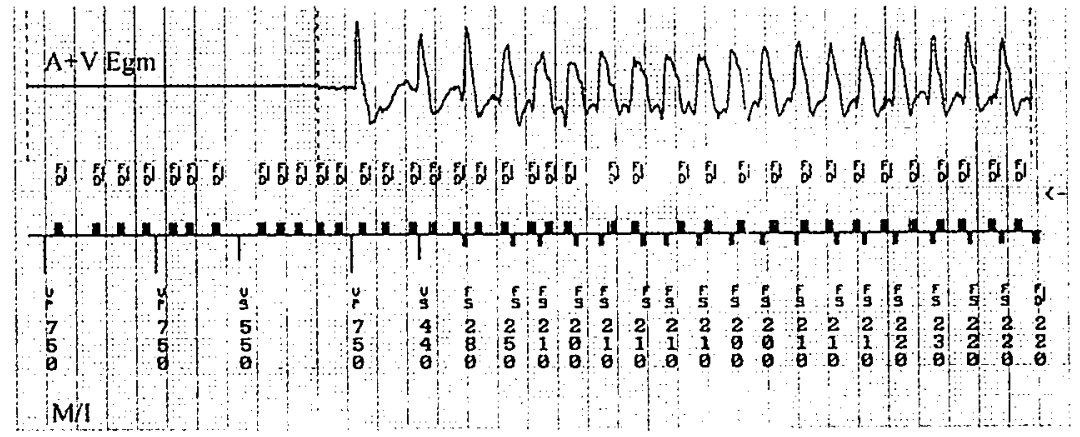

Figure 3. Initiation of VF or VT during atrial arrhythmias was most often preceded by a rapid ventricular rate $(A)$; a regular but not fast ventricular rate $(B)$; RR variations with "short-long-short" sequence $(C)$ were less common. Arrhythmia Logbook indicated that the atrial arrhythmias were present for several hours to days before ventricular arrhythmias occurred. 

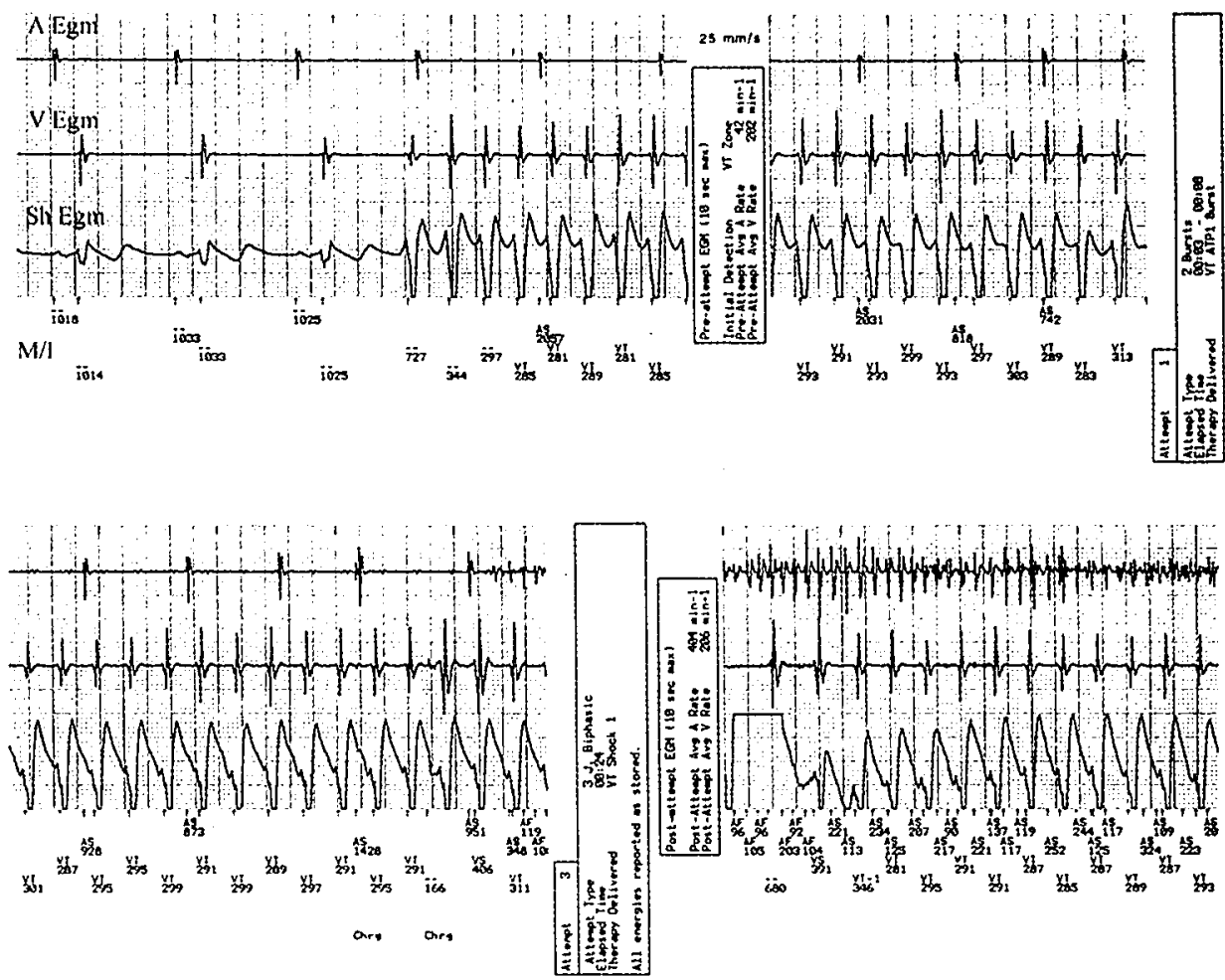

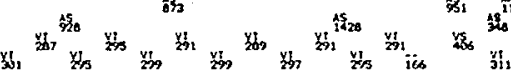
are one

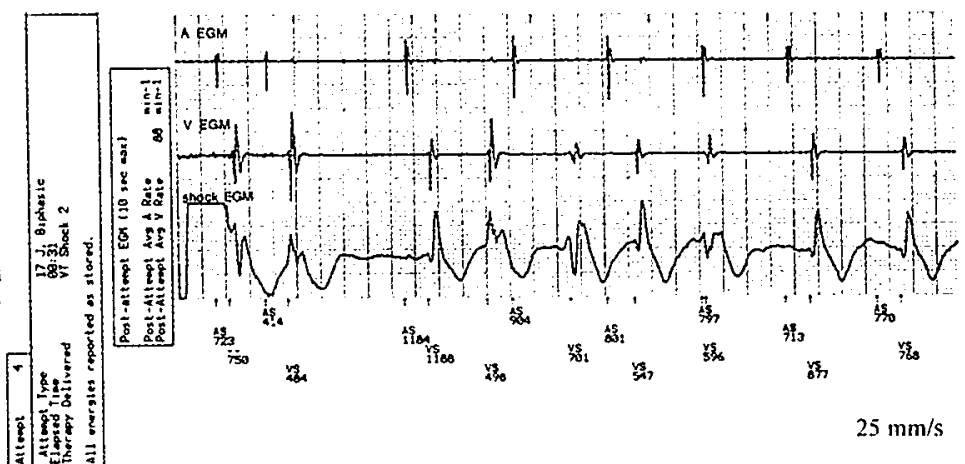

Figure 4. Atrial fibrillation onset during VT without VA conduction. Upper panel: the onset of VT with VA dissociation. Middle panel: AF starts during charging for the first cardioversion therapy. Lower panel: both arrhythmias stop following the second cardioversion. 
shock Egm morphology would be insufficient to differentiate between ventricular and supraventricular rhythm, especially when the ventricular rhythm is fast and regular or after a cardioversion (fig 5). Correct diagnosis of shock induced AF was important to limit device proarrhythmia by adjustments of programming measures or medication.

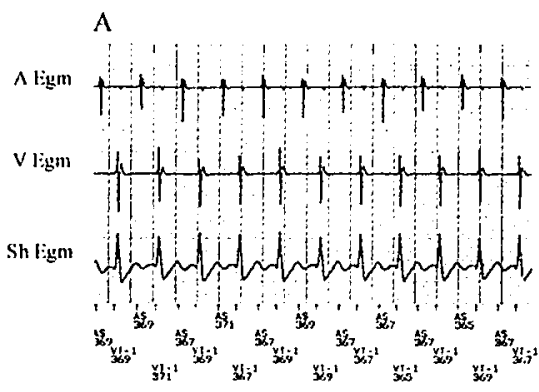

B

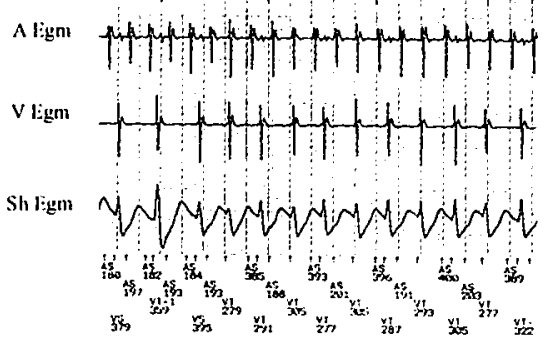

D
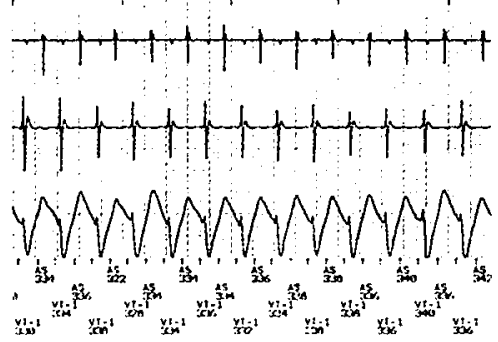

$25 \mathrm{~mm} / \mathrm{s}$

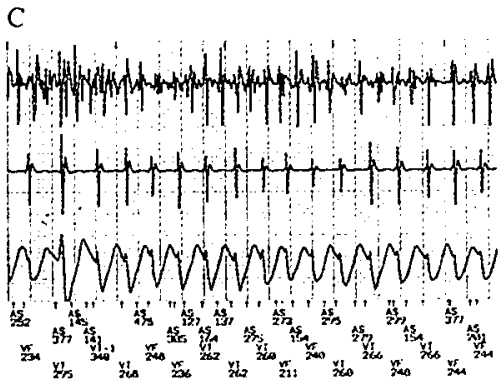

Figure 5. Shortcomings of single chamber V Egm and Shock Egm registration in interpreting the type of arrhythmia. All four Egm snapshots are from the same arrhythmia episode. The morphology of the Shock Egm changes due to rate increase $(A \rightarrow B)$ and cardioversion $(B \rightarrow C \rightarrow D)$. Classifying the arrhythmia using single chamber $V$ and Shock Egm only, may be difficult when the ventricular rate is fast and regular. It is the atrial channel that discloses the presence of a rapid atrial tachycardia (B), atrial fibrillation (C), sinus tachycardia (D).

Sinus tachycardia developing after the onset of ventricular arrhythmia

Sinus tachycardia developing during ventricular arrhythmia could be diagnosed only in VTs without retrograde conduction, which was the case in 708/724 episodes (fig 1 B). All 47 fast VT and 661 VT episodes were accompanied by VA dissociation. In $168 / 708(23 \%)$ of these episodes A Egm showed sinus tachycardia developing after the onset of ventricular arrhythmia. Sinus tachycardia was observed during 3/47 (6\%) episodes of fast VT and during 165/661 (25\%) episodes of VT. 
A subgroup of 5 patients experienced during follow-up a high incidence (more than $1 /$ day) of recurrent monomorphic VT responding poorly to previously successful antitachycardia pacing therapy. The VTs had CL ranging from 340-560 msec, were mostly asymptomatic, sometimes causing only minimal symptoms of "heart working differently". It was the cardioversion that made the patients aware of the arrhythmia. No change in blood pressure could be measured during these VTs while in hospital. In one patient the blood pressure during VT was higher than during sinus rhythm. These 5 patients were on amiodarone and in functional class I -II with a left ventricular ejection fraction of less than $35 \%$ after an extensive myocardial infarction. In spite of good haemodynamic tolerance, the intracardiac A Egm showed the development of a sinus tachycardia during VT. Increase of the initial dose of the beta-blocker (2 patients carvedilol, 3 patients metoprolol) 1.5-2 times did not reduce the frequency of VTs. Eventually propranolol (160-320 $\mathrm{mg}$ /daily) was prescribed; in 2 patients amiodarone was discontinued. The change in antiarrhythmic drug resulted in reduction of VT recurrences to less than $1 /$ month in 4 patients and disappearance of VTs in 1 patient. In addition, the recurrent VTs could now successfully be terminated with pacing therapies. VTs in patients in whom amiodarone was stopped showed clearly shorter CL but much easier pace-termination and no need for cardioversion (fig 6). The haemodynamical tolerance of these VTs remained unchanged good.

\section{Ventricular arrhythmia rate and ICD detection zones}

Dual chamber Egm documentation of VT detection and redetection revealed that in $59 / 724(8 \%)$ in 10 patients ventricular arrhythmia CL was at the edge or outside the programmed detection zone. In 44 cases the VT fell out of the detection zone due to deceleration after (pacing or cardioversion) therapy. Changes in rate and morphology of single chamber V Egm after ventricular therapy suggested VT termination in most cases. In some patients differences between V Egm morphology during VT and sinus rhythm were more discrete than during two different VTs (fig 7). It was the A Egm, which allowed information about VA dissociation.

The $\mathrm{VV}$ and $\mathrm{AA}$ vs time interval plots with their graphical display add very clear insight in the arrhythmia - ICD interaction and cover a longer time than the Egm registration. The interval plots allow not only information about VT at the moment of detection and therapy (fig 8). The interval plots can disclose within one arrhythmia episode presence of multiple VTs with different CL, before or after the treated VT was documented in Egm (fig $8 \mathrm{~A}$ ). The interval plots depict also VT dynamics that are useful for programming therapy. VTs showing a gradual rate acceleration might benefit from antitachycardia pacing applied early after onset (fig $8 \mathrm{~B}$ ). In cases where an arrhythmia shows only small rate fluctuations, the relation between AA and VV interval plots makes differentiation between VT and sinus tachycardia easier (fig $8 \mathrm{C}, \mathrm{D}$ ). It was 


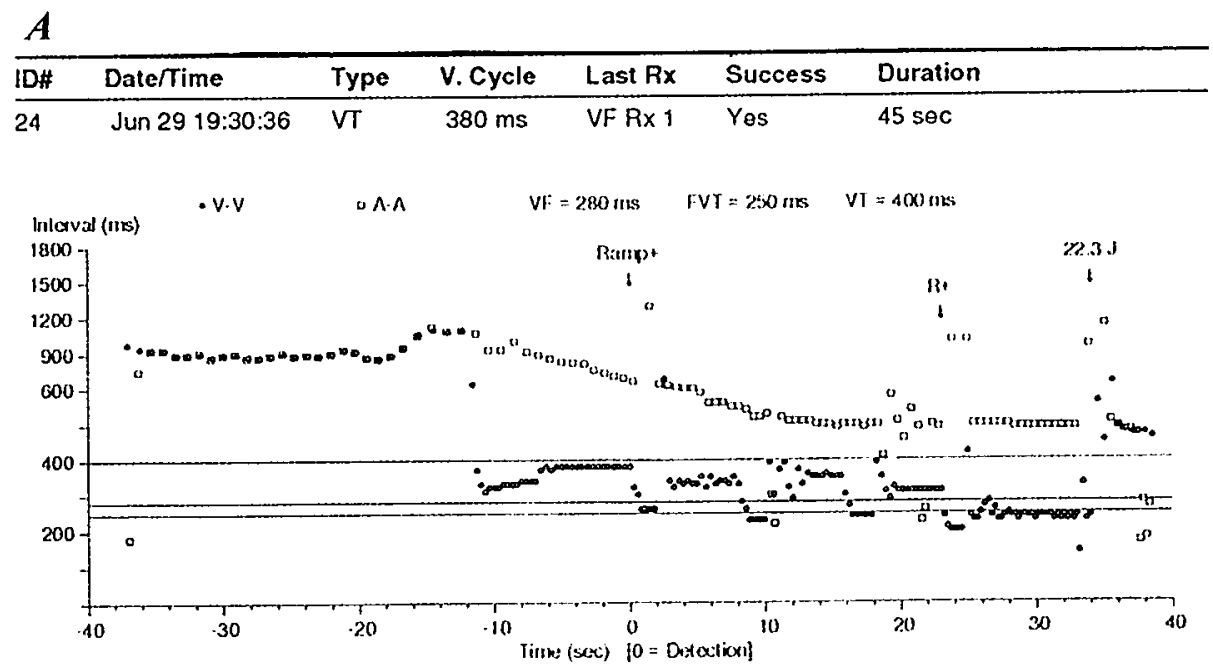

\begin{tabular}{lllllll}
\hline$B$ & & & & \\
\hline ID\# & Date/Time & Type & V. Cycle & Last Rx & Success & Duration \\
\hline 88 & Sep 05 11:44:41 & VT & $310 \mathrm{~ms}$ & VT Rx 1 & Yes & 13 sec
\end{tabular}

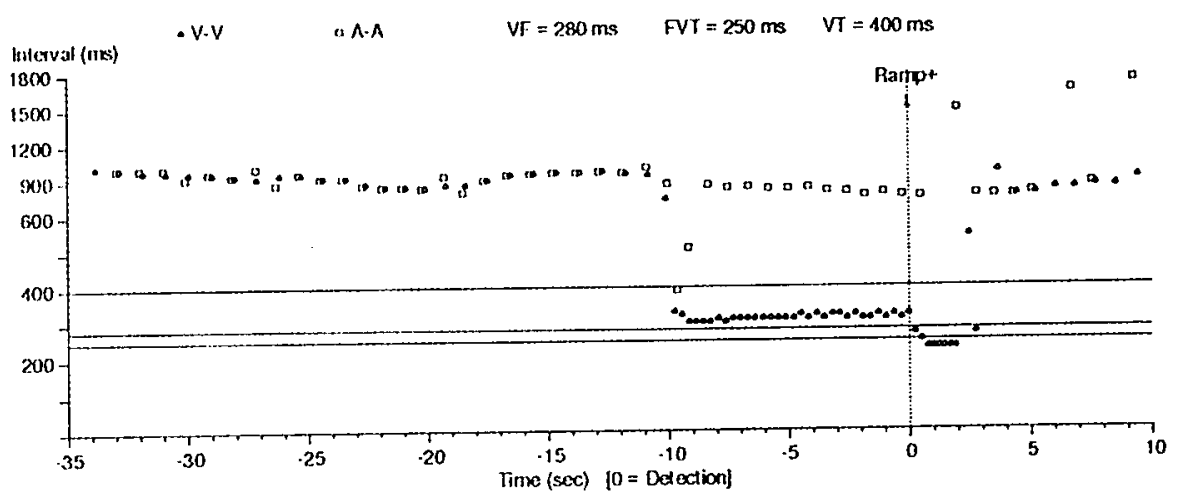

Figure 6. Patient with sinus tachycardia developing during VT before (A) and after (B) discontinuation of amiodarone and change of another beta-blocker to propranolol. A) There is no sinus tachycardia before the onset of VT but it develops thereafter. One of the unsuccessful sequences of antitachycardia pacing causes acceleration of the VT into VF zone triggering (successful) defibrillation shock. B) Same patient 2 months after the change in medication. The VT now has a 70 msec shorter $C L$ but can easily be terminated with the first attempt of antitachycardia pacing. 

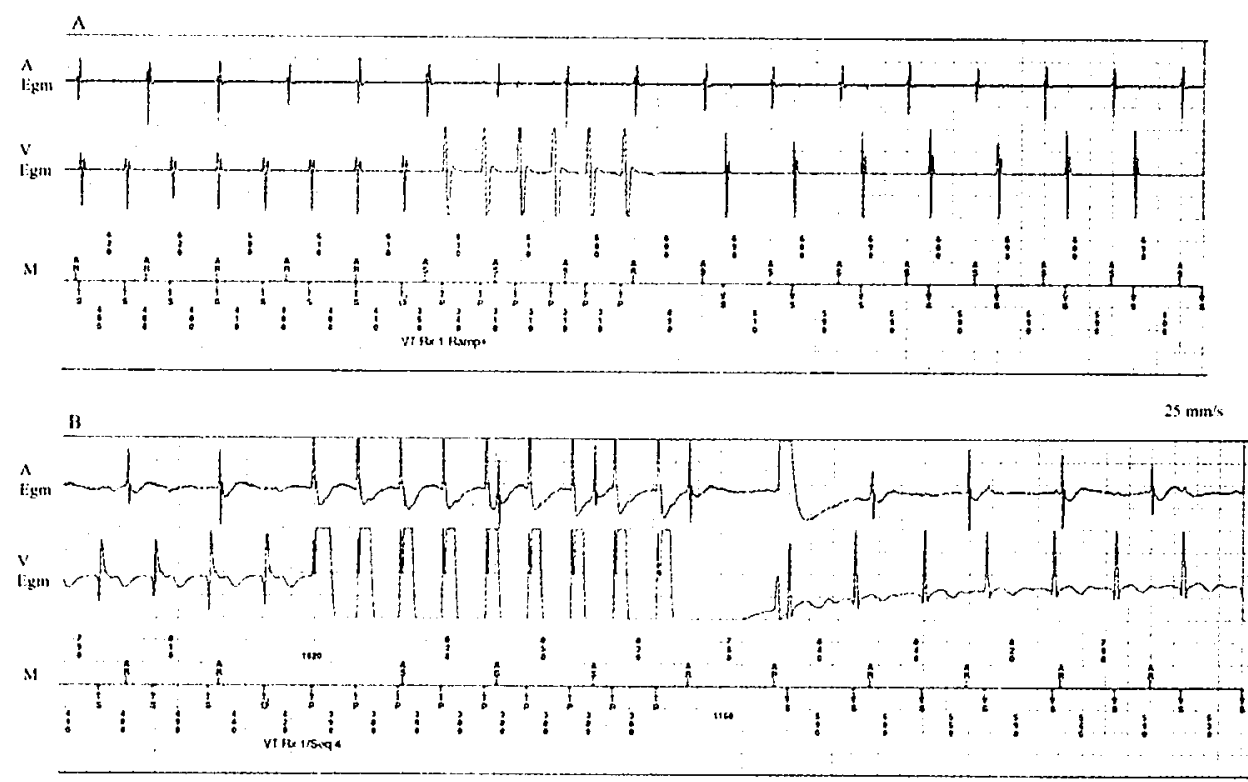

Figure 7. Use of dual chamber Egm documentation to judge VT therapy outcome and to correct programming of detection zones. A) Following pacing therapy for VT there is sinus rhythm. B) Following antitachycardia pacing there is no termination but another VT occurs with a 100 msec longer $\mathrm{CL}$ and $\mathrm{VA}$ dissociation. The differences in $\mathrm{V} \mathrm{Egm} \mathrm{morphology} \mathrm{between} \mathrm{VT} \mathrm{and} \mathrm{the} \mathrm{conducted}$ sinus beats (A) are more discrete than between the two VTs (B). Without A Egm the outcome in $B$ ) might be misinterpreted.

particularly useful in slower VTs having V Egms morphology similar to V Egm during sinus tachycardia. Rate decrease following antitachycardia pacing might cause the therapy to be falsely declared successful. However, the AA interval plots with the gradual acceleration of the sinus rate, starting from VT onset, provide indication for unsuccessful pacing therapy (fig $8 \mathrm{C}$ ). In contrast, sinus tachycardia in the VT zone shows also flat or only gradually changing VV interval plots, however with atrial and ventricular events being associated (fig $8 \mathrm{D}$ ).

In all cases of an arrhythmia with 1:1 AV/VA relation the dual chamber Egm registration is indispensable to differentiate sinus tachycardia and VT with 1:1 VA conduction. Both can be present and even may have similar CL in the same patient (fig $1 \mathrm{~A}$ ). 

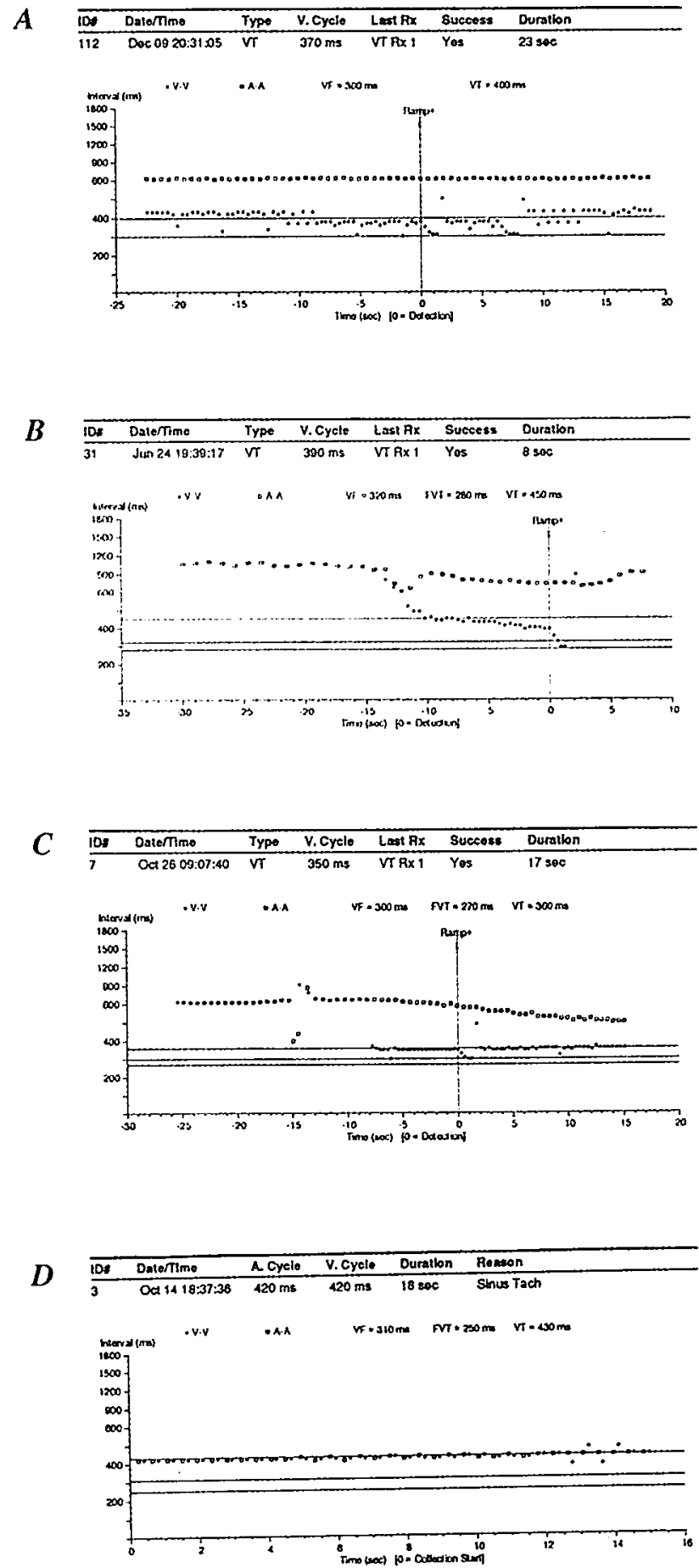

For legend, see next page. 
Figure 8. The value of dual chamber interval plots to evaluate the arrhythmia-ICD interaction. A) VT with two different $\mathrm{CL}$ and VA dissociation. The rate of the first VT (VTa) is outside the detection zone but it accelerates spontaneously (VTb). The VTb is detected and treated with antitachycardia pacing. Following the pace therapy the VT does not stop but the increase in $\mathrm{CL}$ places the VT again outside the detection zone just as VTa. The ventricular rate based single chamber criteria limit the diagnosis to one VTb of only $23 \mathrm{sec}$ duration and considers the therapy successful. It is the A Egm and the evidence of VA dissociation throughout the whole tracing, which help correct interpretation. B) VT with gradual shortening of the CL. The VT started approximately $12 \mathrm{sec}$ before the antitachycardia pacing, showing at that time a CL of 600 $\mathrm{msec}$ and VA dissociation. After $8 \mathrm{sec}$ within the detection zone there is successful pacing therapy and $\mathrm{AV}$ reassociation. In the absence of the $A$ Egm the gradual rate increase from 100 beats/min $\rightarrow 155$ beats $/ \mathrm{min}$ might have given the impression of sinus tachycardia. C) VT with $\mathrm{CL}$ just within the detection zone. $\mathrm{A}$ minimal increase in $\mathrm{CL}$ after antitachycardia pacing causes the false conclusion of therapy success. A Egm shows VA dissociation with increasing rate of sinus tachycardia. D) Sinus tachycardia within the VT detection zone correctly diagnosed by the dual chamber discrimination criteria. In the 1:1 AV associated rhythm the dual chamber Egm registration is helpful to exclude VT with 1:1 retrograde conduction.

\section{DISCUSSION}

As shown, compared with a single chamber system the dual chamber ICD has more to offer than DDD pacing capabilities and VT: SVT arrhythmia discrimination algorithms. The atrial channel registrations are helpful to learn about mechanisms contributing to the onset and the maintenance of ventricular arrhythmias. Dual chamber diagnostics allow useful information about arrhythmia-ICD interaction. That information is helpful to optimize ventricular arrhythmia therapy.

\section{Atrial and ventricular arrhythmia}

Studies on the initiation of ventricular arrhythmias in ICD patients used criteria of timing and morphology of initiating beats registered at the ventricular level in the stored Egm (2). However what appears as a premature event in the V Egm, can be either a ventricular beat or an atrial conducted one. The V Egm morphology of ventricular and supraventricular beats can be very similar, while both normally and aberrantly conducted atrial premature beats are capable of initiating ventricular arrhythmia. The single chamber Egm registration may not be sufficient to trace the chamber of origin of beats initiating VT. This is of relevance for rate stabilization algorithms that were developed to prevent onset of recurrent arrhythmias by premature beats. Value of algorithms may be limited if rate stabilization pacing is not applied in the chamber where premature beats originate.

Initiation of VT and VF by supraventricular beats has been reported in spontaneous and induced arrhythmias (3-7). In our patients ventricular arrhythmia initiated by atrial arrhythmias represent only a relatively small part of all dual chamber documented VT/VF episodes. In the majority of ventricular arrhythmias starting during atrial arrhythmia, a fast ventricular rate was present, only in a few cases a short-long-short 
sequence at the ventricular level was observed. In Jewel AF patients in whom the pacing mode is DDD with mode switching, the ventricular rate stabilization feature can prevent longer RR intervals. Ventricular arrhythmias beginning during AF with a fast ventricular rate indicate that titration of beta-blocking agent might need adjustments during follow-up.

In three cases in which the onset of the atrial arrhythmia was closely followed by the onset of ventricular tachycardia there was a combination of both, a fast and an irregular ventricular rate. In patients in whom bradycardia pacing was programmed in the DDD mode, the fast ventricular rate is caused by tracking of the atrial arrhythmia before mode switching has been effectuated. In addition, the pacemaker AV Wenckenbach may lead to a long-short sequence of the RR interval. Both pacing related phenomena, ventricular rates faster than during spontaneous AV conduction and artificial RR interval irregularities were observed in our patients. To avoid these undesired phenomena in current ICDs one may program the bradycardia pacing in $\mathrm{DDI}(\mathrm{R})$ mode or use fallback pacing. However these options also have their disadvantages. DDD mode with beat -to- beat mode switching together with non-competitive atrial pacing would be better in patients in whom atrial arrhythmias shortly after their onset initiated ventricular arrhythmias.

\section{Sinus tachycardia preceding ventricular arrhythmia initiation}

As documented in the dual chamber Egm, sinus tachycardia was the most frequently present supraventricular tachycardia preceding the onset of VT, in spite of the use of beta-blocking agents in $90 \%$ of our patients. Exercise testing is among the best tools to study exercise related arrhythmias but it cannot mimic all situations patients will encounter in real life. Absence of ventricular arrhythmia during sinus tachycardia on exercise testing (performed twice, the first time being before ICD implantation) was reproducible among two exercise tests but not predictive for daily life situations. Exercise, especially when accompanied by a "target" (catching a bus, playing a game) or emotion (anger or fear) was followed by VT also in patients in whom VT was absent during exercise testing in hospital. Some patients do not recall physical exercise when VT occurred during sinus tachycardia. Increasing the dose of beta-blocking agents helped to reduce the number of sinus tachycardia related VTs during follow-up in only 2 patients. Other causes of sinus tachycardia than physical exercise may be suspected, such as mental stress or autonomic influences (8).

\section{Sinus tachycardia developing after the onset of ventricular arrhythmia}

Ventricular tachycardia, both spontaneous and paced, causes an increase in sympathetic activity in the human heart $(9,10)$. Increased sympathetic activity plays a role in 
preventing arrhythmia related hypotension. Use of beta-blocking agents in $90 \%$ of our patients limits the possibility to follow spontaneous autonomic influences during ventricular arrhythmias. However, sinus tachycardia developing during VT was observed more often during haemodynamically stable VT than during fast VT.

Dual chamber Egm documentation allowed the identification of a subgroup of patients showing development of sinus tachycardia during VT that could not be explained by a fall in blood pressure during the arrhythmia. In these patients the VTs accompanied by gradually increasing sinus rate of pace-termination. The administration of an aselective beta-blocker propranolol resulted in a significant reduction of VT incidence and a response to pace therapy of $100 \%$, also when amiodarone was stopped. In spite of the fact that a sinus tachycardia was no longer present and that the VTs had shorter CL in some patients, haemodynamic tolerance did not worsen. The sinus tachycardia must have other cause than compensating for blood pressure drop.

The beneficial effect of beta-blocking agents on reducing the number of ICD shocks has been reported before in patients with the first generation ICD (11). However limited diagnostics and programmability in those devices did not allow evaluating what the mechanism was of the decreased number of shocks in beta-blocker users. Combining amiodarone with beta-blocking agent has been described to diminish the occurrence of ventricular arrhythmias $(12,13)$. However in many studies prescribing beta-blockers was often limited to certain types of ventricular arrhythmias (exercise and ischemia related or induced by isoproterenol administration) and certain types of patients (using class I or III antiarrhythmic drugs, having congestive heart disease). In our patients the information derived from dual chamber ICD diagnostics helped to guide antiarrhythmic treatment. The advantages of individualizing beta-blocking therapy would not have been suspected from a single chamber V Egm.

\section{Ventricular arrhythmia rate and ICD detection zones}

Information about clinically documented and induced ventricular arrhythmias was a good basis for programming the detection zones, the spontaneous VTs tending to be slower than the induced ones. However some of our patients presented during follow-up new ventricular arrhythmia, different from those documented prior to implant. This was particularly the case in patients with a complex anatomic arrhythmogenic substrate, like right ventricular dysplasia, dilated cardiomyopathy, or in patients after repeated ablations of VTs after an old myocardial infarction.

Dual chamber Egm documentation was very valuable to evaluate arrhythmia detection and redetection especially when VT CL and morphology altered during one episode. Presence of multiple VTs within one detected episode might be inapparent in a single chamber V Egm or Shock Egm registration. A change in V Egm morphology 
together with rate increase before a treated VT might be interpreted as an arrhythmia initiation; respectively rate decrease after a treated $\mathrm{VT}$ as an arrhythmia termination. Without A Egm ventricular arrhythmia detection and redetection would have been speculative in some patients with multiple arrhythmias. The dual chamber diagnostics allow correct interpretation of arrhythmia and therapy outcome. Even when the initial device-based classification is wrong, improved possibilities of verification allow better adjustment of programming measures.

\section{CONCLUSIONS}

The diagnostic possibilities in the dual chamber ICD allow better insight in the mechanisms contributing to the onset and maintenance of ventricular arrhythmias. That knowledge is far from complete and we are unable to influence all the different factors at the present time. However the currently available possibilities helped in some patients to influence positively the ventricular arrhythmia occurrence and its therapy. Sinus tachycardia was the most often occurring supraventricular arrhythmia either preceding or accompanying ventricular arrhythmias. VT initiation preceded by sinus tachycardia was much more often observed in ambulatory patients than during exercise testing in hospital. This could only to a limited extent be influenced by beta-blocking agents. In a subgroup of patients sinus tachycardia developing after the onset of a sustained monomorphic VT did not seem to be related to haemodynamic instability but rather to an exaggerated sympathetic response. In these patients VT incidence and amenability for pace-termination were positively influenced by propranolol. VTs arising during atrial arrhythmias were usually preceded by a high ventricular rate and not by short-long-short sequences in the ventricle. In spite of the wide use of beta-blocking agents the atrial and ventricular Egm registration suggested that the dose was too low in many patients. The dual chamber diagnostics provide useful information about arrhythmia detection and monitoring of therapy outcome, especially in patients with multiple VTs. This allows better evaluation of arrhythmia-ICD interaction and adjustments of programming parameters.

The advantages of dual chamber systems go further than avoidance of what was considered to be the shortcomings of single chamber system. They result in better treatment of the ventricular arrhythmia itself. 


\section{REFERENCES}

1. Fan K, Lee K, Lau CP. Dual chamber implantable cardioverter defibrillator benefits and limitations. J Interv Card Electrophysiol 1999; 3:239-45

2. Roelke M, Garan H, McGovern BA, Ruskin JN. Analysis of the initiation of spontaneous monomorphic ventricular tachycardia by stored intracardiac electrograms. J Am Coll Cardiol. $1994 ; 23: 117-22$.

3. Wellens HJ, Bar FW, Farre J, Ross DL, Wiener I, Vanagt EJ. Initiation and termination of ventricular tachycardia by supraventricular stimuli. Am J Cardiol. 1980; 46:576-82.

4. Belhassen B, Shapira I, Kauli N, Keren A, Laniado S. Initiation and termination of ventricular tachycardia by supraventricular beats. Basic Res Cardiol 1980:75:772-9

5. Berman ND. Ventricular tachycardia initiated by both normally and aberrantly conducted atria premature beats. PACE 1980; 3:519-25.

6. Bekheit S, Turitto G, Fontaine J, el-Sherif $N$. Initiation of ventricular fibrillation by supraventricular beats in patients with acute myocardial infarction. Br Heart J. 1988;59:190-5.

7. Brembilla-Perrot $B$, Gretzinger A, Guenoun P, Giorgi JP, Licho T. Initiation of ventricular fibrillation by atrial fibrillation. Eur Heart J. 1994; 15:289-91.

8. Pruvot E, Thonet $G$, Vesin J, et al. Heart rate dynamics at the onset of ventricular tachycardias as retrieved from implantable cardioverter-defibrillators in patients with coronary artery disease. Circulation 2000; 101:2398-2404.

9. Smith M, Ellenbogen K, Beightol L, Eckberg D. Sympathetic neural responses to induced ventricular tachycardia. J Am Coll Cardiol 1991; 18:1015-24.

10. Smith M, Joglar J, Wasmund S, et al. Reflex control of sympathetic activity during simulated ventricular tachycardia in humans. Circulation. 1999; 100:628-634.

11. Leclerq JF, Leenhart A, Coumel P, Slama R. Efficacy of beta-blocking agents in reducing the number of shocks in patients implanted with first-generation automatic defibrillators. Eur Heart J 1992; 13:1180-1184.

12. Tonet J, Frank R, Fontaine $G$, et al. Efficacy and safety of low doses of beta-blocker agents combined with amiodarone in refractory ventricular tachycardia. PACE 1988; 11:1984-1989.

13. Ogunyankin K, Singh B. Mortality reduction by antiadrenergic modulation of arrhythmogenic substrate: significance of combining beta blockers and amiodarone Am J Card 1999; 84: 76-82 
CHAPTER 5

\section{Diagnosis and Therapy of Atrial Tachyarrhythmias in the Dual Chamber Implantable Cardioverter Defibrillator}

Barbara Dijkman MD, Hein J.J.Wellens MD Department of Cardiology

Academic Hospital Maastricht, Maastricht, The Netherlands

Published: Journal of Cardiovascular Electrophysiology 2000;11:1196-1205. 


\section{ABSTRACT}

Backgromnd: Devices capable of monitoring and treating atrial tachyarrhythmias provide information about the natural history of the arrhythmias and are potentially able to influence their natural course by electrical therapy early after onset.

Methods and results: Types of atrial arrhythmia and efficacy of device therapies were evaluated in 30 patients implanted with the 7250 Jewel AF Medtronic ICD model. All patients had structural heart disease and documented sustained ventricular and atrial arrhythmias (27 patients AF) prior to implant. Twenty patients were on amiodarone, three on sotalol. During $20 \pm 10$ months follow-up 600 atrial arrhythmia recurrences were documented in $50 \%$ of patients. AF was diagnosed in $19 \%$, fast polymorphic AT in $20 \%$, fast monomorphic AT in $57 \%$, slow AT in $4 \%$ of episodes. The two adaptive pacing therapies Burst and Ramp, together with the $50 \mathrm{~Hz}$ burst were successful in $57 \%$ of detected atrial arrhythmias. Burst and Ramp were responsible for $49 \%$ and 50 $\mathrm{Hz}$ burst for $51 \%$ of successfully treated arrhythmias; $33 \%$ of episodes terminated spontaneously. No ventricular proarrhythmia was observed due to atrial pacing therapies. In $30 \%$ episodes dual chamber pacing was required due to post termination bradycardia. Atrial arrhythmia recurrences in patients with dilated cardiomyopathy were not amenable for pacing therapies. Several aspects of atrial arrhythmia diagnosis, therapy and documentation that are specific for functioning of Jewel AF are discussed. Conclusions: Atrial arrhythmias in ICD patients with diseased hearts and on class III antiarrhythmics had frequently a longer cycle length than AF. Half of these arrhythmias could be terminated with pacing therapies, one third terminated spontaneously. 


\section{INTRODUCTION}

Devices capable of monitoring atrial tachyarrhythmias with atrial fibrillation as the most important one, allow following the natural history of the arrhythmias over a longer period of time in the same patient. Better understanding of the mechanisms of different types of atrial arrhythmias will help to develop better therapy strategies and in some patients possibly also prevention. Successful treatment early after onset might potentially change the natural history of atrial arrhythmias and reduce their morbidity. The purpose of this study was to evaluate types of atrial arrhythmia and efficacy of device therapies in patients with a history of both ventricular and atrial tachyarrhythmias treated with the Medtronic 7250 Jewel AF model of an implantable cardioverter defibrillator (ICD).

\section{METHODS}

\section{Patients}

The Medtronic 7250 Jewel AF ICD was implanted in 30 patients (59 \pm 14 years old) with documented ventricular and atrial tachyarrhythmias. The ICD indication was ventricular fibrillation (VF) in 11 patients, haemodynamically unstable ventricular tachycardia (VT) in 13 patients, and both VF and VT in 6 patients. All patients had at least one documented episode of paroxysmal sustained atrial tachyarrhythmia: 17 patients atrial fibrillation (AF), 1 patient atrial flutter (AFL), 7 patients both $A F$ and AFL, 2 patient atrial tachycardia (AT), 2 patients both AF and AT, 1 patient $A F$ and AV nodal tachycardia. Altogether AF was documented in 27 patients. Ten of them experienced at least one persistent episode of AF, which did not terminate within one or more days after onset and required cardioversion to obtain sinus rhythm. The history of atrial tachyarrhythmias was present during $24 \pm 19$ months prior to implant. The arrhythmia etiology was coronary artery disease in 22 patients, idiopathic dilated cardiomyopathy in 4 patients, hypertrophic obstructive cardiomyopathy in 1 patient, myotonic dystrophy in 1 patient, aortic valve disease in 1 patients and surgically corrected Fallot's tetralogy in 1 patient. Ten patients were in functional class III NYHA, 15 in class II and 5 in class I. The mean left ventricular ejection fraction was $36 \pm 15 \%$, the left atrial diameter $50 \pm 7 \mathrm{~mm}$. Twenty-five patients were on a beta-blocking agent, 20 patients were on amiodarone, 3 patients on sotalol, 5 patients on digitalis and 1 patient on verapamil. 21/23 patients on class III antiarrhythmics were using the drug at the time atrial arrhythmia was documented before ICD implantation. Prior to implant atrial tachyarrhythmias were accompanied by palpitations in 2 patients, pump failure in 5 patients, tiredness and decrease of exercise tolerance in 7 
patients, were asymptomatic or terminated before symptoms occurred in the remaining patients.

Jewel AF atrial arrhythmia management.

The Jewel AF has two separately programmable detection zones for atrial arrhythmias. The median atrial interval is the basis to classify the rhythm as AF or AT. It is possible to program the detection zones with an overlap; classification of an atrial arrhythmia detected in that area depends on rate regularity (fig 1A). The detection timer has a not programmable initial part and a programmable sustained part. The initial AF/AT detection criterion is fulfilled if the atrial arrhythmia is present during 32 ventricular cycles. The sustained detection timer can be set to 0 minutes. Treatment of atrial arrhythmias starts after the programmable sustained duration timer has expired. During detection the Jewel AF continuously evaluates the presence of atrial sensed events between RR intervals and adjusts its AF/AT evidence counter (fig 1B). The counter increments by one if there are $\geq 2$ atrial events between $R R$ intervals, remains the same in the presence of one event with a 1:1 AV relation and decrements on each subsequent 1:1 AV related event. Once the initial detection criterion is met, it remains satisfied when AF/AT evidence counter is $\geq 27$.

The Jewel AF has three modalities of atrial therapy: 1) Adaptive antitachycardia pacing (ATP) of Burst and Ramp type, 2) A $50 \mathrm{~Hz}(20 \mathrm{msec})$ atrial burst pacing, and 3) Cardioversion. All three therapy modalities are available in AT, the last two in AF. Within one detected type of arrhythmia therapies are delivered according to increasing aggressiveness $(\mathrm{ATP} \rightarrow 50 \mathrm{~Hz}$ burst $\rightarrow \mathrm{CV}$ ) but it is possible to skip some steps. The ATP and $50 \mathrm{~Hz}$ burst therapies can consist of a programmable number of burst sequences (1).

Programming settings - atrial arrhythmia detection, therapy and documentation

Detection. The detection zones were programmed individually in each patient based on information on spontaneous and induced atrial arrhythmia. The sustained detection timer was programmed to " 0 " to allow information about type of arrhythmia shortly after onset.

Thernpies. The AT pacing therapies were programmed in the following order: 1) Burst, 2) Ramp, 3) $50 \mathrm{~Hz}$ burst. The initial ATP parameters (5-7 sequences of 5-7 stimuli with a coupling interval of $81 \%$ of the tachycardia interval) were adjusted during follow-up depending on their efficacy. Most often it was necessary to shorten the coupling intervals and to add more stimuli. Bursts of $50 \mathrm{~Hz}$ were given in 3-5 sequences of 1 second duration for AT and of 2-3 seconds for AF. The $50 \mathrm{~Hz}$ burst was 


\section{A. Atrial Fibrillation and Atrial Tachycardia Detection}

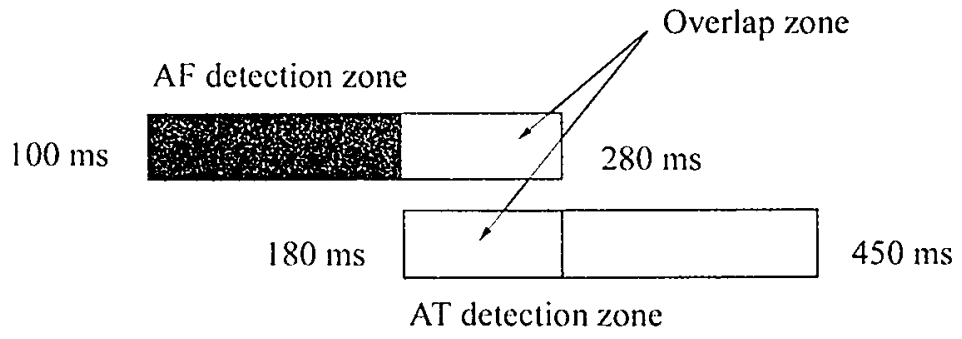

B. AF Evidence Counter

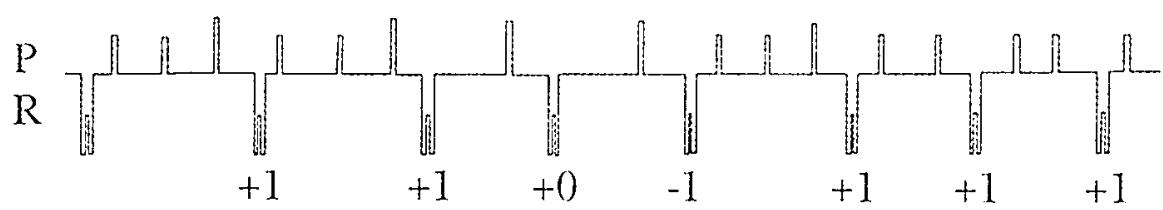

Figure 1. The atrial arrhythmia detection criteria are based on rate and pattern information. A) AF/AT detection zones; arrhythmia detected in the overlap zone is classified as AF or AT depending on rate regularity; $B$ ) AF/AT evidence counter increments in the presence of $=2$ atrial sensed events within one ventricular RR interval; it decreases in the presence of a 1:1 relation between atrial and ventricular events; it remains unchanged the first time a 1:1 AV relation is encountered.

the only therapy automatically allowed in AF. If one type therapy was more effective than the others, steps of therapy were adjusted to use the more successful type. Cardioversion was applied only as manual therapy in hospital by physician using short-acting anesthetic agents.

Documentation. Atrial arrhythmias and their therapies were evaluated in spontaneous episodes in which documentation of intracardiac electrograms (Egm), marker annotations $(M)$ and interval data was available. The Egm source for data collection was the wideband "Atip-Vrig" stored for 5 seconds prior to the first atrial therapy. 


\section{Definitions of atrial arrhythmia types and therapy outcome}

The classification of atrial arrhythmias used in this study was based on device provided information about median PP interval, Egm pattern and in fast atrial arrhythmias detected in AF/AT overlap zone also PP regularity. Atrial arrhythmia was considered $A F$ if the $\mathrm{P}$ waves were polymorphic, the isoelectric line absent or irregular and the median PP interval $\leq 240 \mathrm{msec}$. Fast AT if the $\mathrm{P}$ waves and an isoelectric line were clearly distinguishable and the median PP interval was 200-300 msec; the P waves could have different morphologies (fast polymorphic $A T$ ) or one morphology (fast monomorphic $A T$ ). Atrial tachycardia was considered slow $A T$ if the median PP interval was $>300$ msec (fig 2). This classification was applied to obtain more accurate arrhythmia diagnosis than the one that is based on rate only.

The Jewel AF provides no Egm registration following consecutive therapies but gives only marker annotation after arrhythmia termination. The marker annotations do not always cover the moment of the last applied therapy, especially when termination did not occur immediately after therapy. Results of therapy were classified as follows: Successful: the arrhythmia is terminated and the last successful therapy sequence or the result of it is documented in the marker annotation. The duration of the whole episode is in accordance with the number of redetections and therapy sequences.

Spontancously terminated: the arrhythmia is terminated and the marker annotation shows the transition from arrhythmia to sinus rhythm without any therapy in between. The termination occurs before programmed therapies were exhausted.

Unsuccessful: the arrhythmia is still present after all programmed therapies had been applied. Some of these unsuccessfully treated arrhythmias stopped spontaneously some hours (-days) later, others were still ongoing during interrogation.

Other therapy ontcome: initiated atrial therapies are withheld because of VT detection (having priority of treatment) or device interrogation, or the arrhythmia was stopped by inappropriate ventricular therapy (cardioversion).

This outcome classification was applied parallel to what is clinically understood under "success" or "failure" of electrical therapy.

Types of atrial arrhythmias and their therapies outcome were analyzed using descriptive statistics.

\section{RESULTS}

During the follow-up period of $20 \pm 10$ (2-35) months atrial arrhythmia recurrences occurred in $15(50 \%)$ patients. All 15 patients were known with AF before implant: in 9 patients AF was the only clinically documented arrhythmia, in 4 patients both $\mathrm{AF}+\mathrm{AFL}$, in 1 patient $\mathrm{AF}+\mathrm{AT}$ and in 1 patient $\mathrm{AF}+\mathrm{AV}$ nodal tachycardia. 

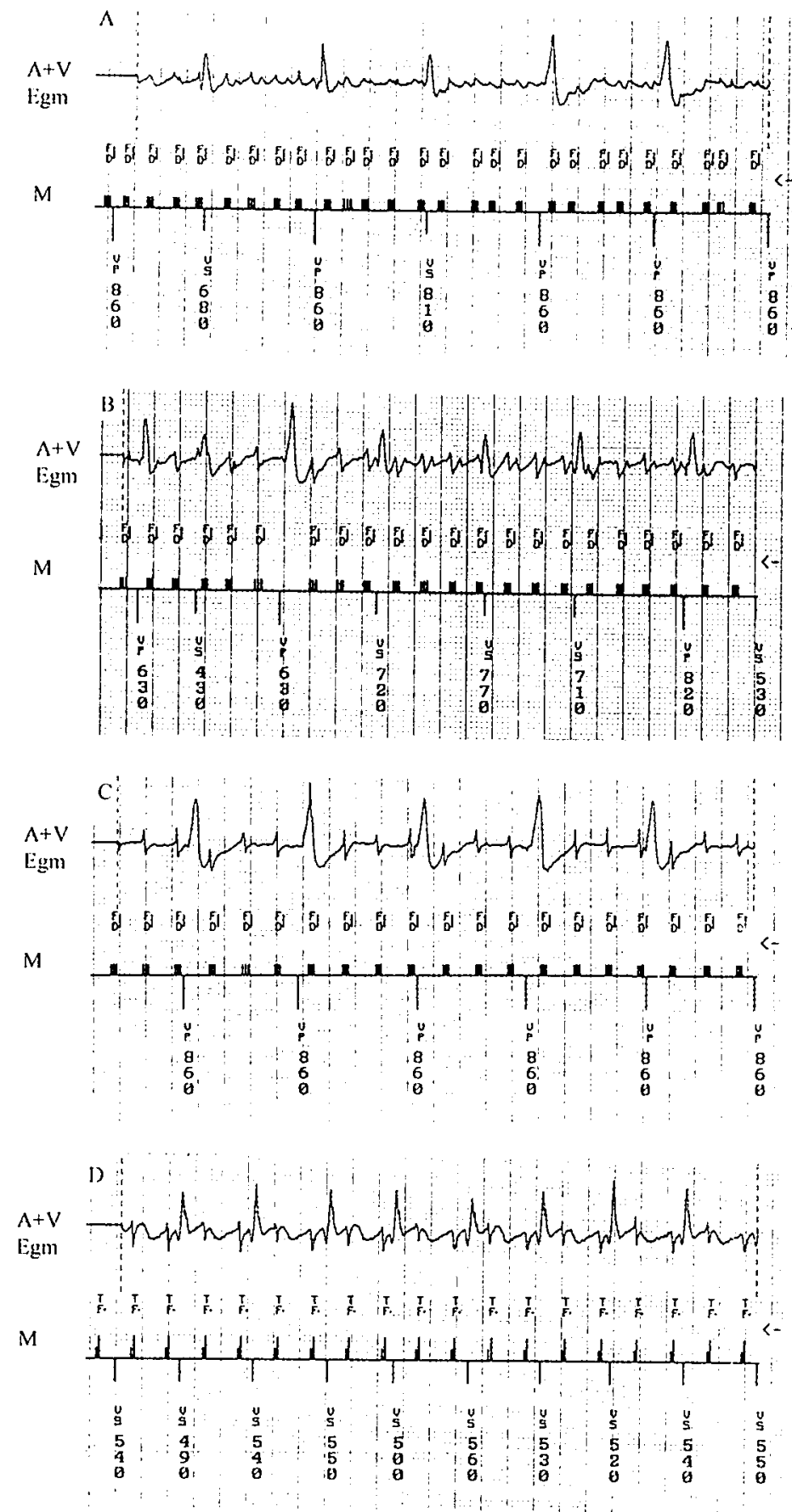

Figure 2. Intracardiac wideband "A tip- $V$ ring" Egm registration early after the onset of an atrial arrhythmia. Added to arrhythmia classification the $P$ wave morphology in: A) AF, B) fast polymorphic AT, C) fast monomorphic AT, D) slow AT. All Egm are $0,5 \mathrm{mV} / \mathrm{mm}$ and $25 \mathrm{~mm} / \mathrm{s}$. 


\section{Types of atrial arrhythmia documented by Jewel AF}

Spontaneous and treated atrial arrhythmias recurrences could in 600 cases be documented with information on median PP interval, Egm pattern, arrhythmia duration and applied therapies. The mean PP interval of all recurrent atrial arrhythmias was 255 \pm 35 (130-360) msec.

Using the criteria described under methods we diagnosed $112(19 \%)$ episodes of AF, $122(20 \%)$ fast polymorphic AT, 345 (57\%) fast monomorphic AT, and 21 (4\%) slow AT. The mean of PP intervals in AF was $215 \pm 25 \mathrm{msec}$, in fast polymorphic AT $240 \pm$ $30 \mathrm{msec}$, in fast monomorphic AT $270 \pm 25 \mathrm{msec}$, in slow AT $320 \pm 20 \mathrm{msec}$.

In 188/600 (31\%) episodes the initial arrhythmia classification by Jewel AF altered within the same episode; for example AT became AF following ATP or AF became AT following $50 \mathrm{~Hz}$ burst. Also spontaneous changes in arrhythmia, like rate deceleration caused changes in classification.

The contribution of AF among all atrial arrhythmias was different in patients with different arrhythmia etiology: $10 \%$ in patients with coronary artery disease and $80 \%$ in patients with dilated cardiomyopathy.

\section{Atrial arrhythmia therapy outcome documented by Jewel AF}

In an attempt to terminate 600 detected atrial arrhythmias the Jewel AF applied in total 3365 sequences of pacing therapies, on average 5,6 sequences per arrhythmia episode: 1165 Burst, $945 \mathrm{Ramp}$, and 1255 of $50 \mathrm{~Hz}$ burst pacing sequences. Taking into consideration the lack of Egm documentation of consecutive redetections and therapies we cannot comment about the efficacy of these "intermediate" therapies and have limited our conclusions to the last applied therapy (fig 3).

Using the criteria described under methods, atrial arrhythmia was treated successfully in $345(58 \%)$ episodes, unsuccessfully in $114(19 \%)$ episodes, terminated spontaneously before the programmed therapies were applied in 97 (16\%) episodes. Therapy outcome was in $8 \%$ classified as "other": atrial arrhythmia detection was suspended due to the VT/VF detection in 28 episodes or interrogation in 8 episodes, arrhythmia was terminated with inappropriate shock in 8 episodes. Most (102/114) of the unsuccessfully treated arrhythmias stopped spontaneously as well after some hours (-days) of duration. Thus, the total number of spontaneously terminated arrhythmias includes 97 episodes in which arrhythmia stopped before the programmed therapies were applied and the 102 episodes in which arrhythmia stopped longer time after the unsuccessful therapies were exhausted.

In 20 episodes $\mathrm{AF}$ was persistent and had to be terminated by manually commanded ICD cardioversion under short acting anesthesia. The in-hospital cardioversions were performed within several days after persistent AF was documented. 


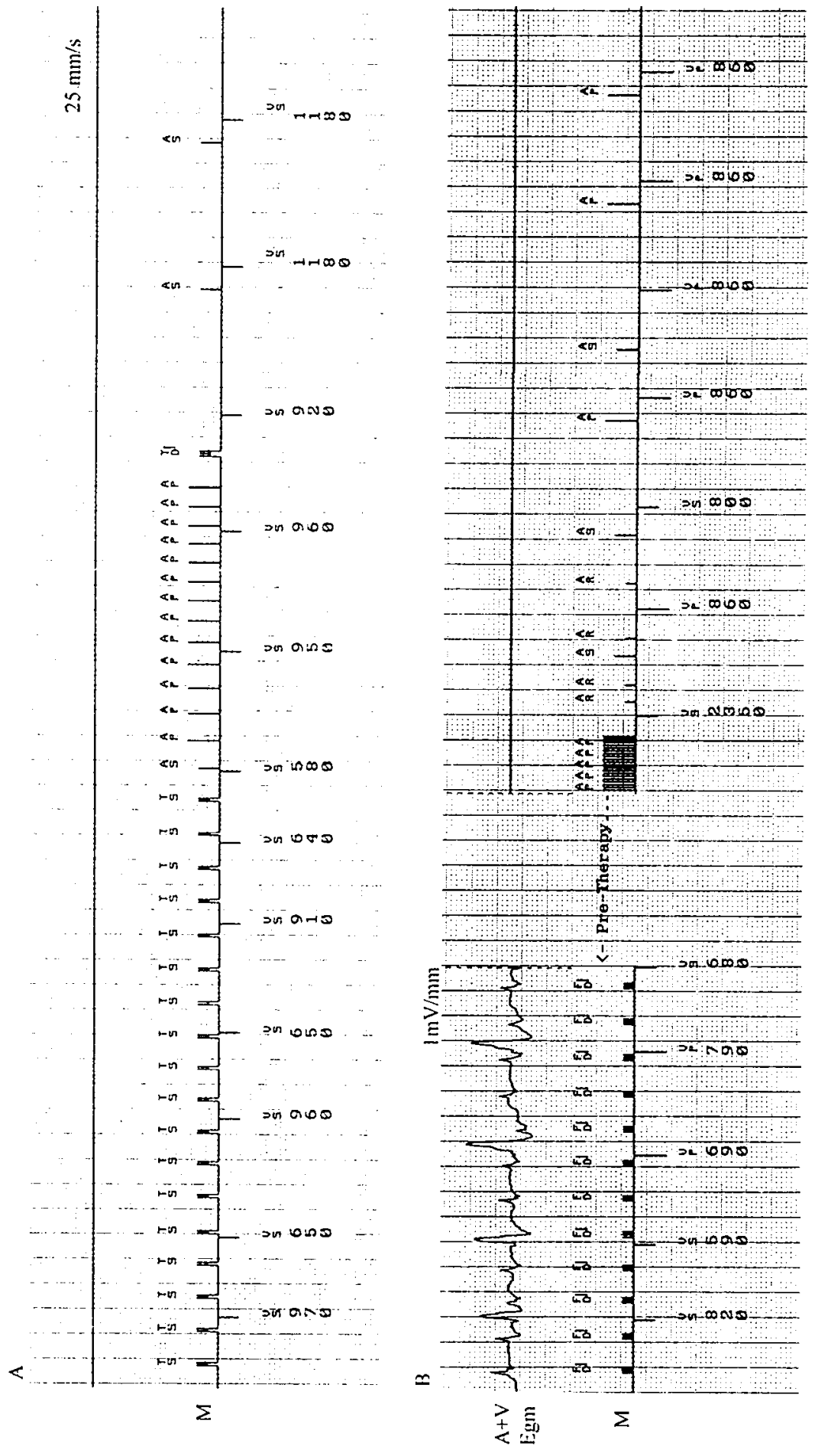



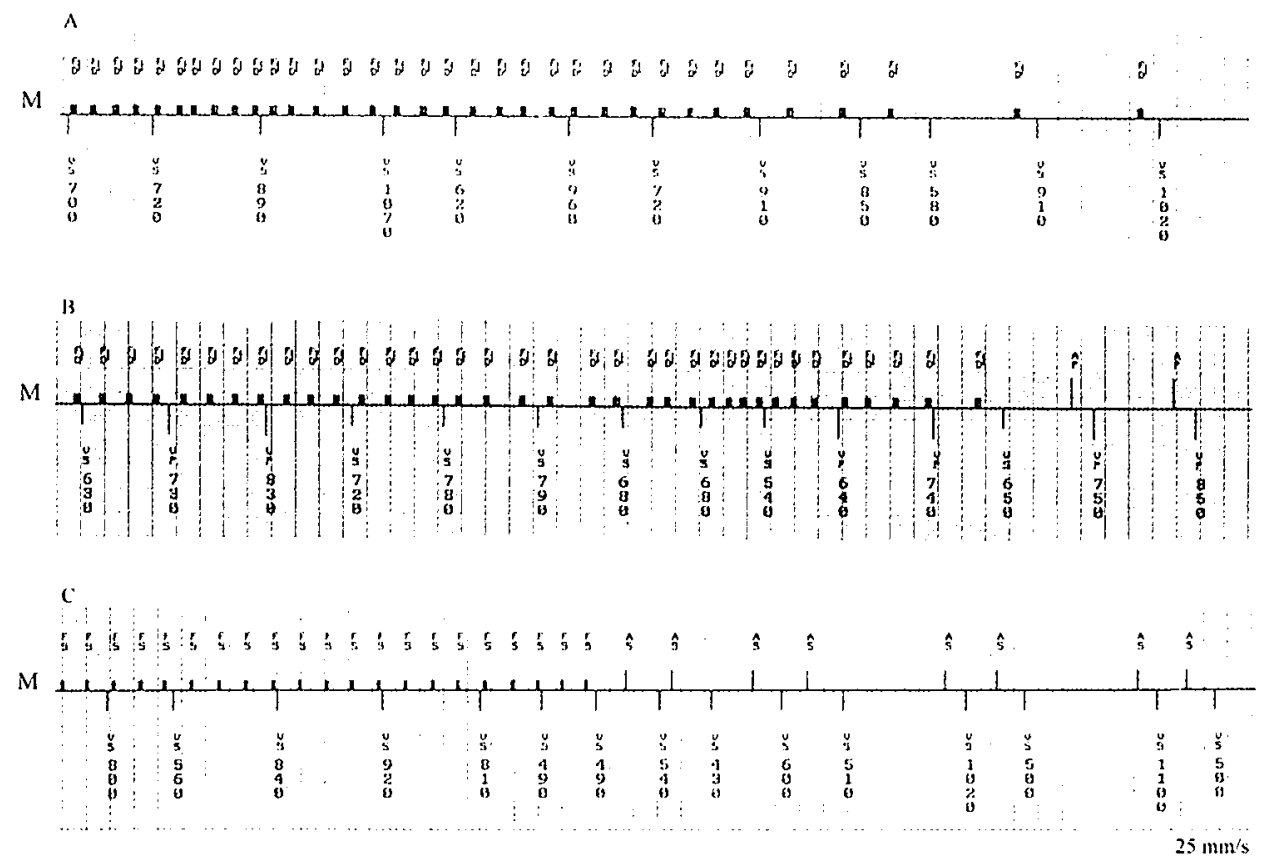

Figure 4. Spontaneous termination of an atrial arrhythmia, showing a pattern of: A) deceleration, B) brief acceleration, $C$ ) no visible changes in $\mathrm{CL}$ before atrial bigeminy starts.

In arrhythmias that were successfully terminated the ATP was responsible for $49 \%$ and the $50 \mathrm{~Hz}$ burst for $51 \%$ of the results.

In $45 \%$ of successfully treated atrial arrhythmias there was immediate pace termination, in the remaining episodes delayed termination.

Atrial arrhythmias that stopped spontaneously showed in $40 \%$ deceleration, in $5 \%$ both acceleration and deceleration and in $56 \%$ no change in CL before termination (fig 4).

The outcome of all pacing therapies combined, in different types of atrial arrhythmia is shown in table 1. The individual contribution of Burst, Ramp and $50 \mathrm{~Hz}$ burst in successful arrhythmia termination is shown in table 2 .

While evaluating performance of atrial therapies by Jewel AF, two aspects of device functioning deserve special attention. Documentation of the moment of applied therapy in the marker channel is provided only following the last sequence of the last therapy. This means that the documentation is available in only $14 \%$ of all therapy sequences, considering the number of episodes and the number of applied sequences. Changes of the arrhythmia classification within one episode can result in a seemingly inconsistent relation between the initially detected arrhythmia and the last therapy, for 
Table 1. Outcome of All ATP and $50 \mathrm{~Hz}$ Therapies Combined in Different Types of Atrial Arrhythmia.

\begin{tabular}{|c|c|c|c|c|c|}
\hline Type of atrial arrhythmia & $\mathrm{Nb}$ & $\begin{array}{l}\text { Successfully } \\
\text { treated }\end{array}$ & $\begin{array}{l}\text { Spontaneous } \\
\text { termination before all } \\
\text { therapies exhausted }\end{array}$ & $\begin{array}{l}\text { Unsuccessfully } \\
\text { treated }\end{array}$ & $\begin{array}{l}\text { Other } \\
\text { outcome }\end{array}$ \\
\hline $\mathrm{AF}$ & 112 & 20 & 30 & 45 & 17 \\
\hline Fast polymorphic AT & 122 & 45 & 24 & 35 & 18 \\
\hline Fast monomorphic AT & 345 & 267 & 38 & 32 & 8 \\
\hline Slow AT & 21 & 13 & 5 & 2 & 1 \\
\hline Total & 600 & 345 & 97 & $114^{*}$ & 44 \\
\hline
\end{tabular}

* $102 / 114$ unsuccessfully treated atrial arrhythmias terminated hours (-days) later; thus, the total number of spontaneously terminated arrhythmias was $97+102=199$.

Table 2. Efficacy of Burst, Ramp, and $50 \mathrm{~Hz}$ Burst as Last Applied Therapy in Successful Treatment of Atrial Arrhythmias.

\begin{tabular}{|c|c|c|c|c|c|}
\hline \multirow{2}{*}{$\begin{array}{l}\text { Atrial arrhythmia-initial } \\
\text { detection }\end{array}$} & \multirow[b]{2}{*}{$\mathrm{Nb}$} & \multirow{2}{*}{$\begin{array}{l}\text { Success three pacing } \\
\text { therapies combined }\end{array}$} & \multicolumn{3}{|c|}{ Success individual therapy } \\
\hline & & & Burst & Ramp & $50 \mathrm{~Hz}$ burst \\
\hline $\mathrm{AF}$ & 112 & 20 & 7 & 1 & 12 \\
\hline Fast polymorphic AT & 122 & 45 & 28 & 7 & 10 \\
\hline Fast monomorphic AT & 345 & 267 & 90 & 25 & 152 \\
\hline Slow AT & 21 & 13 & 9 & 2 & 2 \\
\hline Total & 600 & 345 & 134 & 35 & 176 \\
\hline
\end{tabular}

example successful Burst for AF. This occurred when the initially detected arrhythmia underwent changes, spontaneous or therapy induced, and became atrial arrhythmia of another type.

Analysis of the therapy sequence suggests that in some cases atrial therapy that was not successful in first instance changed the type of arrhythmia allowing termination with another type of therapy in second instance. In 38 cases the $50 \mathrm{~Hz}$ burst did not terminate the initial arrhythmia (usually fast AT, often classified by the device as AF) but caused deceleration so that it was next detected as AT and successfully terminated with ATP. In 19 cases aggravation of arrhythmia occurred - the initially detected AT changed into AF following burst pacing.

The ability to terminate or influence atrial arrhythmia using various pacing therapies was observed only in patients with coronary artery disease and valvular heart disease but never in patients with dilated cardiomyopathy. Also the percentage of spontaneously terminating arrhythmias was much higher in patients with coronary artery disease than in patients with cardiomyopathy ( $35 \%$ vs $4 \%$ ). 

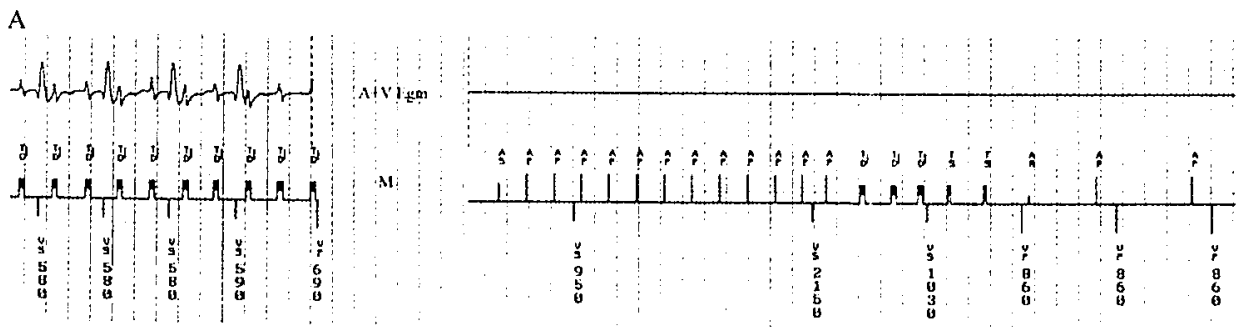

B
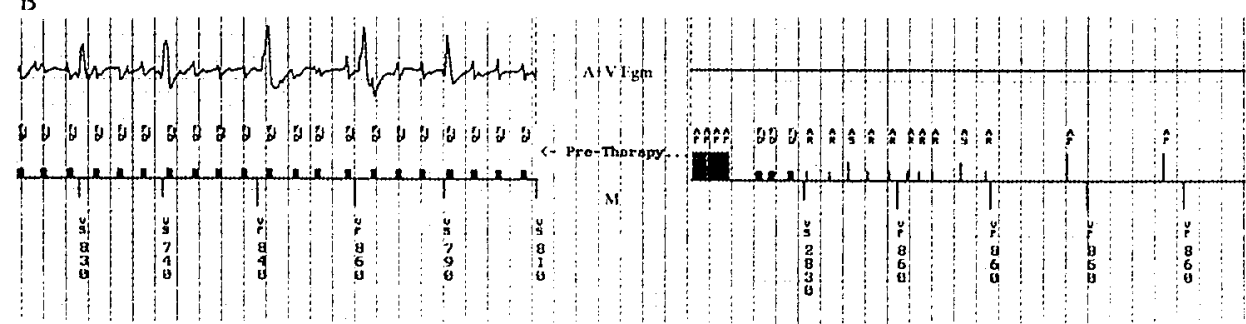

Figure 5. Ventricular bradycardia during atrial pacing therapies and post-termination dual chamber pacing. The Egm registrations in both left panels show an atrial arrhythmia with normal AV conduction. The only cause for ventricular pacing in B left is ventricular rate stabilization. A) During ATP (atrial rate only $20 \%$ faster than during AT) there is a suppression of $A V$ conduction. B) During $50 \mathrm{~Hz}$ burst there is $>2.5 \mathrm{sec}$ pause in RR interval. Following atrial arrhythmia termination there is pacing in atrium and ventricle.

There was no single case of ventricular proarrhythmia observed due to atrial therapies. In $200(33 \%)$ of episodes dual chamber pacing was present because of bradycardia following both spontaneous and therapy-induced terminations. Prolonged $(>2.5 \mathrm{sec})$ suppression of AV conduction during therapy application was seen rarely, almost exclusively during $50 \mathrm{~Hz}$ burst (fig 5 ).

\section{DISCUSSION}

Types of atrial arrhythmias documented by Jewel AF

Despite the fact that AF was clinically documented in all patients prior to ICD implant, it was identified in only $19 \%$ of recurrent atrial arrhythmias. The most frequently occurring was fast AT, both polymorphic and monomorphic. Some of the fast monomorphic AT were presumably AFL, but the single channel Egm registration does not allow verifying this presumption. The incidence of an arrhythmia which (at 
onset) could be qualified as AF was much lower than expected in this group of patients before ICD implant. This might be due to different reasons.

In patients who do not recognize the initiation of their atrial arrhythmias, an Ecg registration might be not representative for the whole scale of atrial arrhythmias that were present in these patients prior to implant. In addition, a pre-implant Ecg registration documents a different moment of arrhythmia than the post-implant Egm registration of the first 15-30 seconds after onset. The coexistence of more than one type of atrial arrhythmias, some of them being asymptomatic, in the same patient has already been reported $(2,3)$ and the activation sequence during spontaneous conversions was described (4).

Class III drugs were used in $93 \%$ and beta-blocking agent in $83 \%$ of patients in this group. Amiodarone might enhance changing $\mathrm{AF}$ to a more organized and slower form of atrial arrhythmias (5) and lengthen the CL of AFL (6). Jayachandran et al (7) showed in an animal study that also propranolol significantly lengthened the AF interval. The effects ascribed to the use of amiodarone were observed in patients with coronary artery and valvular heart disease. However there was a trend towards different type of atrial arrhythmias recurrences in patients with dilated cardiomyopathy as compared to coronary artery disease. patients with dilated cardiomyopathy had less frequently recurrences but of longer duration, less spontaneous terminations and less organized type of arrhythmias.

Although high-resolution mapping gives detailed insights in the activation patterns of atria during $\mathrm{AF}(8)$, it cannot be used to evaluate recurrent arrhythmia in the clinical setting. The criteria used by Wells based on the morphology of a single bipolar atrial electrogram (9) are more readily applicable to classify arrhythmia in devices. The Egm registrations of atrial arrhythmias in the Jewel AF have positive and negative features. Positive is, that the device offers to select one of the four sources of the atrial Egm. Considering the necessity to collect information about the ventricular rhythm we chose for the wideband A tip-V ring. Registering the atrial Egm only is also possible. Due to the fact that the atrial therapies were programmed to be delivered without delay in our patients, the registered Egm represents the arrhythmia early after its onset. In addition, the Egm registrations are comparable in different episodes because they are derived from a stable atrial lead. However there are also limitations inherent to the stable position of one intracardiac lead. The Egms derived from it cannot be representative for the whole electrical activity of both atria. In the study on atrial electrograms on transition between AFL and AF Emori et al (10) found that during AF the electrograms from the atrial septum showed a disorganized and fractionated pattern, while the electrograms from the right atrial free wall remained organized with sharp deflections and an isoelectric line. The negative side of Egm collection is its short duration and presence only on initial detection. These limitations of device diagnostics must be considered while interpreting atrial arrhythmias. 
Some of the pitfalls of the device based arrhythmia detection in Jewel AF are shown in fig 6. The AF/AT evidence counter "pools" information from the 27 most recent RR intervals in order to be sure that the arrhythmia remains sustained. That definition of sustained arrhythmia may not allow recognizing bouts of non-sustained arrhythmias with few sinus beats among them (fig 6A). Once the AF evidence counter is satisfied it may be difficult to "get rid" of arguments for an arrhythmia in the presence of sinus rhythm with atrial bigeminy or short non-sustained AT (fig 6B). Calculating the median of atrial intervals does not give insight in temporary rhythm accelerations/decelerations and is insufficient without the Egm for conclusions about the type of atrial arrhythmia (fig $6 \mathrm{C}$ ). Another feature of device based arrhythmia detection, making it different from that during the electrophysiological study, is the presence of blanking periods. Atrial sensed blanking of $100 \mathrm{~ms}$ assures detection of the presence of an arrhythmia. The cross-chamber blanking of $30 \mathrm{~ms}$ during ventricular paced events may periodically obscure events on the atrial channel. Blanking some of the fibrillation waves, can influence value of PP intervals and rate regularity (fig $6 \mathrm{D}$ ). Intermittent atrial undersensing (fig $6 \mathrm{E}$ ) during AF may also influence interpretation of arrhythmia. The value of the median PP intervals is the basis for ICD arrhythmia diagnosis. The average of the PP intervals is the basis for calculation of coupling interval and interval decrement in adaptive pacing therapy. Regularly blanked or undersensed $P$ waves might result in detection of arrhythmia with a longer CL or in delivery of pacing therapies with to long coupling intervals and to slow interval decrements. These aspects of atrial arrhythmia detection in Jewel AF were one of the reasons to add the Egm pattern to device based rate classification.

\section{Atrial arrhythmia therapy outcome documented by Jewel AF}

The most frequently recurring arrhythmias had at their onset more degree of organization and a longer CL than AF. These fast AT were in half of the cases pace-terminable. The efficacy of (last applied) pacing therapies in all documented episodes was comparable in ATP with the $50 \mathrm{~Hz}$ burst. However, when evaluating performance of the three types of therapies separately, their availability in different types of arrhythmias with different "degrees of difficulty" must be considered. The $50 \mathrm{~Hz}$ burst is available only in AF and as the third therapy tire in AT, so it would not readily be used for easy pace-terminable arrhythmias.

In the small subgroup of fast AT that were classified as AF, the first therapy, the $50 \mathrm{~Hz}$ burst, did not terminate the arrhythmia itself but influenced it in such a way that it was thereafter amenable for ATP. The lack of Egm registrations of intermediate stages of therapy sequences does not allow insight into the exact mechanism, how the $50 \mathrm{~Hz}$ burst modifies the initial arrhythmia. 

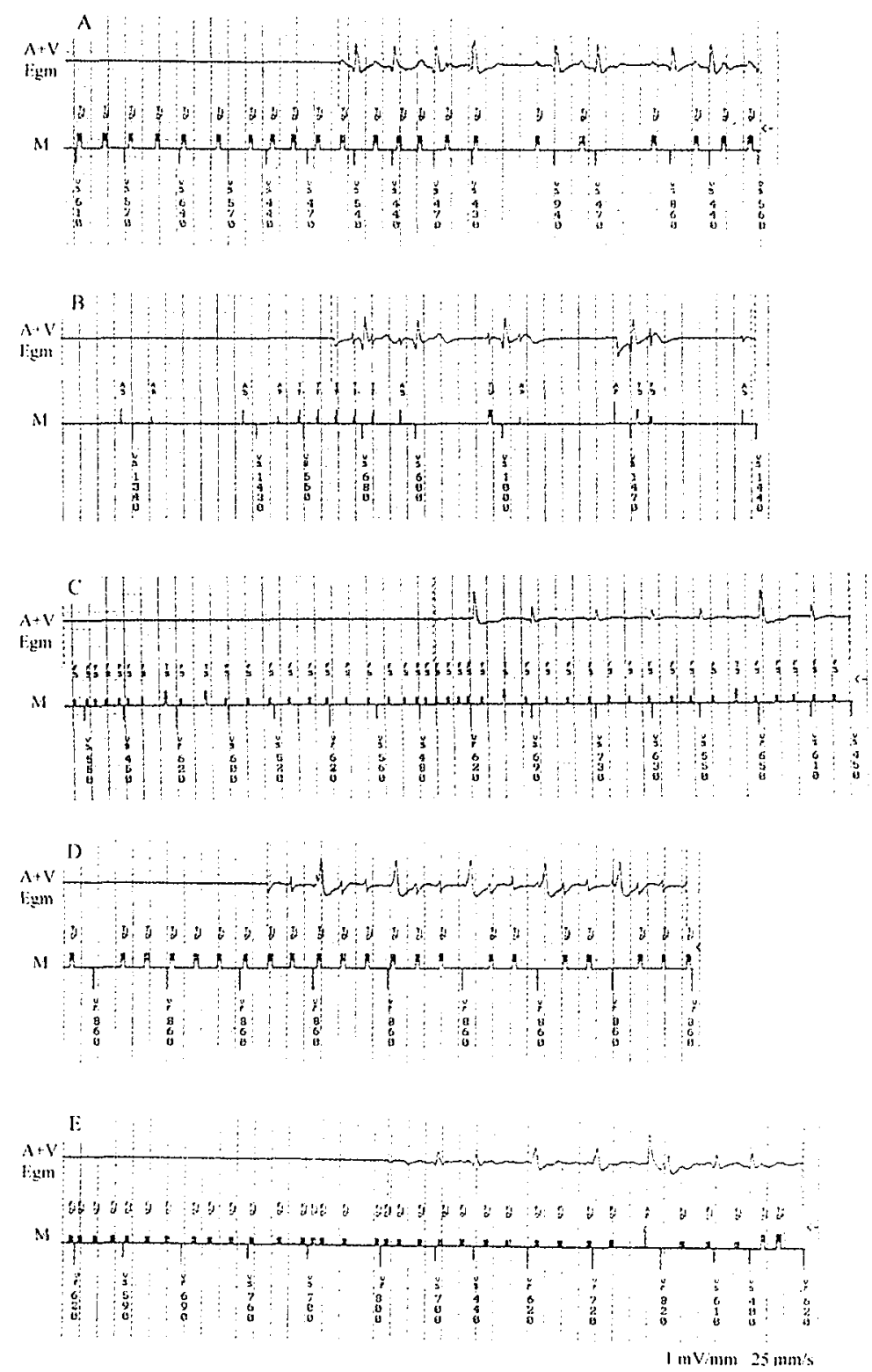

Figure 6. Importance of adequate Egm documentation for atrial arrhythmia diagnosis: A) Inertion of the AF/AT evidence counter does not allow detection of brief spontaneous terminations. B) Once the arrhythmia has been detected but is no longer present, the AF/AT evidence counter cannot decrease to less than 27 in the presence of atrial bigeminy and non-sustained AT. C) Fluctuations in rate and regularity of the arrhythmia are of importance when the final classification: AF or AT is made. D) The cross-chamber blanking during ventricular paced events covering atrial sense events may influence mean PP interval and rate regularity. E) Intermittent atrial undersensing during AF might delay detection of sustained arrhythmia. 
The value of amiodarone as antiarrhythmic drug for AF also in patients with congestive heart failure is supported by the results of the CHF-STAT study (11). Except from effect of amiodarone on reduction of AF recurrences, it can facilitate spontaneous termination of AF. Also the newer class III agent Dofetilide was reported to be effective in suppressing CHF related AF by preventing its recurrences and converting it to SR (12). We could not test the value of class III antiarrhythmics for arrhythmia recurrences since we cannot be sure about the real number of arrhythmia episodes before ICD implantation and post implantation there was no control group without drugs. However, the observations in our group of patients suggest, that the ability to stop arrhythmia with pacing and the high number of spontaneous terminations might be facilitated by the use of amiodarone.

The role of timing of atrial therapies being applied early after onset as protection against development and persistence of AF needs further evaluation.

The accuracy of therapy outcome classification in the Jewel AF depends on the accuracy of arrhythmia detection and redetection. In order to declare arrhythmia termination 5 consecutive sinus (or atrial paced) beats must be present. In a number of situations the device had problems classifying the therapy result (fig 7). The therapy outcome was "unknown" if VT was detected before atrial arrhythmia was teminated (fig 7A); in that case VT correctly received priority in treatment and atrial therapies were suspended. The inability to recognize spontaneous termination (fig $7 \mathrm{~B}$ ) and to bring the AF evidence counter down due to atrial premature beats or non-sustained arrhythmia (fig 7 C) was mainly responsible for not suspending therapy. Atrial arrhythmias that on redetection have slower CL, falling out of the detection window, can also be responsible for an "unknown" therapy classification or very long redetection time (fig $7 \mathrm{D}$ ). All these possible arrhythmia-device interactions make the interpretation of the outcome of the intermediate therapies without Egm registration speculative. Although atrial arrhythmias seldom require urgent interventions, information about these interactions should be more accurate to help in guiding therapy.

\section{LIMITATIONS OF THE STUDY}

This is a study in a small group of patients with ventricular and atrial arrhythmias of various etiologies and an even smaller subgroup of patients actually having recurrences of atrial arrhythmias. The fact that the most patients did not recognize the onset of atrial arrhythmias prior to implant means, that the Ecg documentation do not represent the whole scale of arrhythmias in these patients. The diagnostics of the Jewel AF do not disclose all information on arrhythmia diagnosis and the efficacy of automatic therapies. 

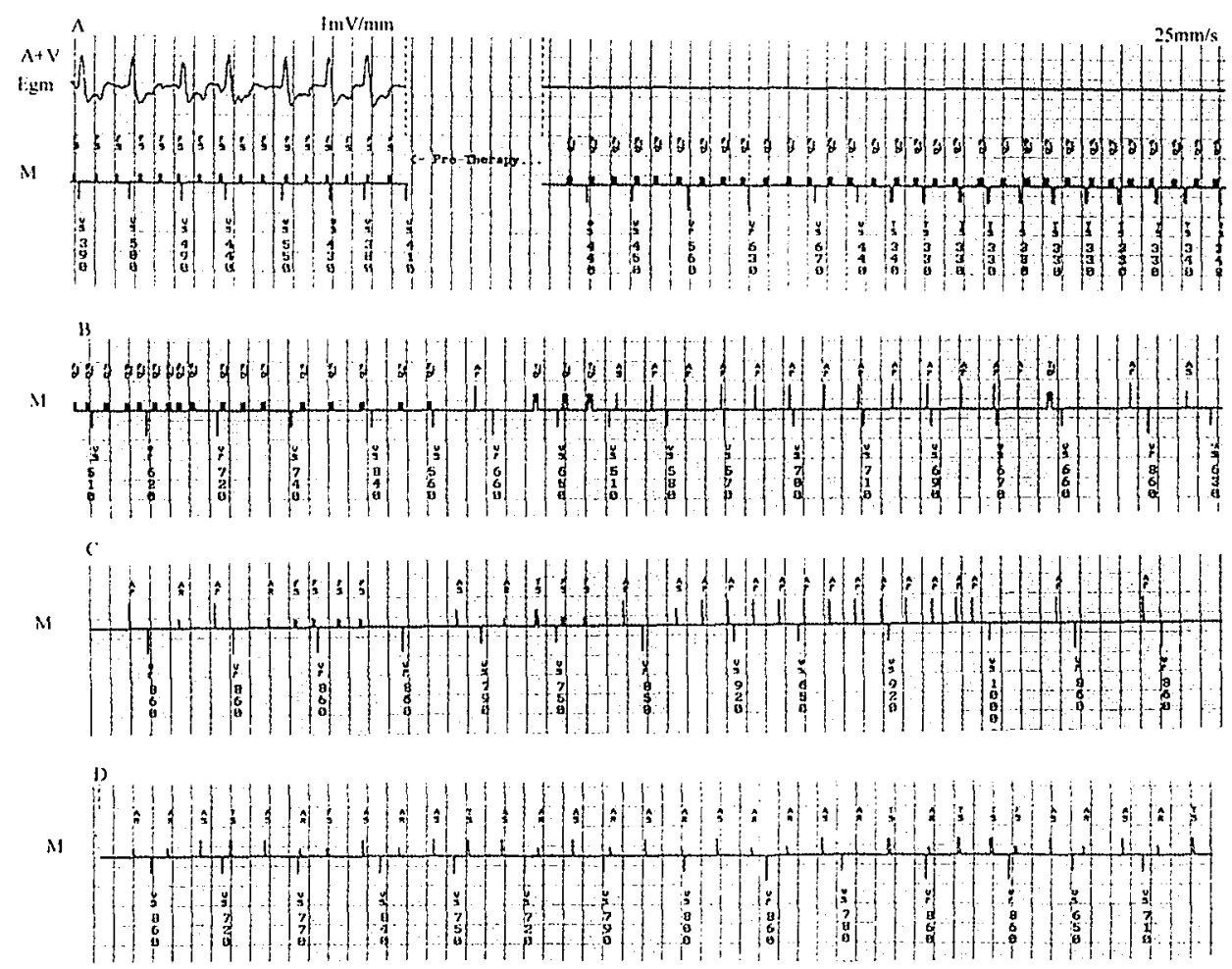

Figure 7. Importance of documenting arrhythmia redetection and termination for therapy result classification. A) Detection of ventricular tachycardia during atrial arrhythmia correctly suspends atrial therapies. The atrial arrhythmia therapy result is labeled as "unknown". B) AF/AT evidence counter did not decrement enough to recognize spontaneous termination. C) Apparent atrial arrhythmia triggers inappropriate atrial therapy. Therapy outcome (ike in $B$ ) and C) was often labeled as successful by the device. D) Following therapy another arrhythmia is detected with a $\mathrm{CL}$ at the edge of the detection zone. It can take long before the device declares the outcome.

\section{CONCLUSIONS}

The Jewel AF is the first dual chamber arrhythmia management device that allows information on atrial arrhythmias and their treatment under ambulatory conditions. Atrial arrhythmias observed in patients with diseased hearts, a history of VT/VF and on class III antiarrhythmics had frequently longer cycle lengths than AF. Half of these arrhythmias could be terminated with pacing therapies, one third terminated spontaneously. The definitions used by the Jewel AF to classify types of atrial arrhythmia and therapy outcome need to be adjusted to bring them closer to the clinical understanding arrhythmia diagnosis and success of electrical therapy. Future refinements of diagnos- 
tics are required to obtain better insight in arrhythmia characteristics and the value of device interventions.

\section{REFERENCES}

1. Jewel AF Model 7250 Arrhythmia Management Device system reference guide. Medtronic Inc, 1996.

2. Page R, Wilkinson W, Clair W, McCarthy E, Pritchett E. Asymptomatic arrhythmias in patients with symptomatic paroxysmal atrial fibrillation and paroxysmal supraventricular tachycardia. Circulation. 1994; 89:224-227.

3. Roithinger F, Lesh $M$. What is the relationship of atrial flutter and fibrillation. PACE 1999; 22:643-654.

4. Roithinger F, Karch R, Steiner P, SippensGroenewegen A, Lesh M. Relationship between atrial flutter in humans. Activation sequence changes during spontaneous conversion. Circulation. 1997; 96:3484-3491.

5. Huang D, Monahan K, Zimetbaum P, Papagcorgiou P, Epstein L, Josephson M. Hybrid pharmacologic and ablative therapy. J Cardiovasc Electrophysiol 1998; 9: 462-469.

6. Stambler B, Wood M, Ellenbogen K. Pharmacological alterations in human type I atrial flutter cycle length and monophasic action potential duration.J Am Coll Cardiol 1996; 27:453-61.

7. Jayachandran V, Sih H, Winkle W, Zipes D, Hutchins G, Olgin J. Atrial fibrillation produced by prolonged rapid atrial pacing is associated with heterogeneous changes in atrial sympathetic innervation. Circulation 2000; 101:1185-1191

8. Kumagai K, Khrestian C, Waldo A. Simultaneous multisite mapping studies during induced atrial fibrillation in the sterile pericarditis model. Circulation. 1997;95:511-521.

9. Wells J, Karp R, Kouchoukos N. Characterization of atrial fibrillation in man: Studies following open heart surgery. PACE 1978; 1:426-438.

10. Emori T, Fukushima K, Saito H, Nakayama K, Ohe T. Atrial electrograms and activation sequences in the transition between atrial fibrillation and atrial flutter. J Cardiovasc Electrophysiol, 1998; 9:1173-1179.

11. Deedwania P, Singh B, Ellenbogen K, Fisher S, Fletcher R, Singh S. Spontaneous conversion and maintenance of sinus rhythm by amiodarone in patients with heart failure and atrial fibrillation. Circulation 1998; 98:2574-2579.

12. Torp-Pedersen C, Moller M, Bloch-Thomsen P, Kober L, Sandoe E, Egstrup K, Ang J, Videbaek, Marchant B, Camm A. Dofetilide in patients with congestive heart failure and left ventricular dysfunction. Danish Investigation of Arrhythmia and Mortality on Dofetilide Study Group. N Engl J Med. 1999; 341:857-65. 


\section{Merits and Limitations of the Mode Switching Rate Stabilization Pacing Algorithms in the Implantable Cardioverter Defibrillator}

Barbara Dijkman MD, Hein J.J. Wellens MD Department of Cardiology

Academic Hospital Maastricht, Maastricht, The Netherlands

Journal of Interventional Cardiac Electrophysiology, in press. 


\section{ABSTRACT}

Background: The 7250 Jewel AF Medtronic model of ICD is the first implantable device in which both therapies for atrial arrhythmias and pacing algorithms for atrial arrhythmia prevention are available. Feasibility of that extensive atrial arrhythmia management requires correct and synergic functioning of different algorithms to control arrhythmias.

Methods and results: The ability of the new pacing algorithms to stabilize the atrial rate following termination of treated atrial arrhythmias was evaluated in the marker channel registration of 600 spontaneously occurring episodes in 15 patients with the Jewel AF. All patients ( $55 \pm 15$ years) had structural heart disease and documented atrial and ventricular arrhythmias. Dual chamber rate stabilization pacing was present in 245 (41\%) of episodes following arrhythmia termination and was a part of the mode switching operation during which pacing was provided in the dynamic DDI mode. This algorithm could function as the atrial rate stabilization pacing only when there was a slow spontaneous atrial rhythm or in presence of atrial premature beats conducted to the ventricles with a normal AV time. In case of atrial premature beats with delayed or absent conduction to the ventricles and in case of ventricular premature beats, the algorithm stabilized the ventricular rate. The rate stabilization pacing in DDI mode during sinus rhythm following atrial arrhythmia termination was often extended in time due to the device-based definition of arrhythmia termination. This was also the case in patients, in whom the DDD mode with true atrial rate stabilization algorithm was programmed.

Conclusions: The rate stabilization algorithms in the Jewel AF applied after atrial arrhythmia termination provide pacing that is not based on the timing of atrial events. Only under certain circumstances the algorithm can function as atrial rate stabilization pacing. Adjustments in availability and functioning of the rate stabilization algorithms might be of benefit for the clinical performance of pacing as part of device therapy for atrial arrhythmias. 


\section{INTRODUCTION}

When medical treatment of atrial fibrillation results in insufficient arrhythmia control or side effects, use of nonpharmacological therapies can be considered. Non-pharmacological atrial fibrillation treatment modalities include methods to shorten its duration (external and internal atrial cardioversion), to control the ventricular rate (His bundle ablation followed by pacemaker implantation) or treatment of possible triggers for atrial fibrillation (like pulmonary vein ablation). Preventive strategies for atrial fibrillation were designed to reduce the number of episodes in patients with frequent arrhythmia recurrences. So far prevention of atrial fibrillation by pacing was addressed by trying to optimize site and rate of atrial stimulation (1-3).

The 7250 Jewel AF Medtronic model of ICD (implantable cardioverter defibrillator) is the first implantable device in which different modalities of atrial arrhythmia treatment can be combined with pacing algorithms for atrial arrhythmia prevention (4). Device therapy using that extensive arrhythmia management requires correct and consonant functioning of all algorithms to control arrhythmia. In case of pacing algorithms it is important that, in patients with an atrial arrhythmia, these algorithms support maintenance of a stable sinus rhythm.

The moment following successful therapy of an atrial arrhythmia deserves special attention as far as the mode of pacing is concerned. After termination of atrial fibrillation the electrophysiologic properties of the atria make them vulnerable to premature beats causing early reinitiation of arrhythmia. The purpose of this study was to evaluate the influence of the new mode switching rate stabilization algorithm on atrial rate stability following termination of atrial arrhythmias in patients with the 7250 Jewel AF.

\section{METHODS}

\section{Patients}

Rate stabilization pacing was evaluated following the termination of spontaneously occurring and treated atrial arrhythmias in 15 patients ( $55 \pm 15$ years) implanted with the 7250 Jewel AF. All patients had documented sustained ventricular and atrial arrhythmias. The ICD indication was ventricular fibrillation in 8 patients, haemodynamically unstable ventricular tachycardia in 7 patients. Atrial arrhythmias were atrial fibrillation in 9 patients, both atrial fibrillation and atrial flutter in 4 patients, atrial fibrillation and atrial tachycardia in 1 patient, atrial fibrillation and AV nodal tachycardia in 1 patient. The arrhythmia etiology was coronary artery disease in 9 patients, idiopathic dilated cardiomyopathy in 4 patients, myotonic dystrophy in 1 
patient and valvular heart disease in 1 patient. The mean left ventricular ejection fraction was $35 \pm 13(17-58) \%$ and the left atrial diameter $50 \pm 8(38-64) \mathrm{mm}$. Eleven patients were on a beta-blocking agent, 11 patients on amiodarone, and 3 patients on sotalol. The follow-up period was $20 \pm 10(2-35)$ months.

\section{Jewel AF atrial arrhythmia management}

\section{Detection and therapy}

The Jewel AF has two programmable detection zones for atrial arrhythmias with the median PP interval as the classifier of atrial fibrillation or atrial tachycardia. The device has three modalities of atrial therapy: adaptive antitachycardia pacing, $50 \mathrm{~Hz}$ burst pacing and cardioversion. Only the first two types of therapy were automatically allowed in our patients. Persistent atrial fibrillation episodes were converted in hospital with manually commanded ICD cardioversion using short lasting anesthesia.

\section{Mode switching}

Mode switching in the Jewel AF is initiated when the duration of the median PP interval becomes less than the programmed upper tracking rate interval during at least 6 RR intervals. The transition from DDD to a non-atrial tracking DDI mode, occurring at the onset of atrial arrhythmia, is called Mode Suritch Delta. The transition back from DDI to DDD mode occurs after the termination of atrial arrhythmia (so during sinus rhythm) and is called the Mode Suitch Fall.

\section{Rate stabilization pacing in the Jewel AF}

The Jewel AF has two types of rate stabilization pacing algorithms (fig 1).

1) Mode switching rate stabilization controls the magnitude of ventricular rate changes from the onset until after the termination of atrial arrhythmia. Increments and decrements of the pacing rate are limited by the rate slope throughout the whole mode switching operation. Mode switching rate stabilization modulates a temporary lower rate in the ventricle using the dynamic DDI mode, so pacing can occur also in the atrium.

The duration of mode switching rate stabilization pacing immediately after atrial arrhythmia termination can be extended with the Mode Switch Duell. During a programmable period of time, overdrive DDI pacing at a rate higher than the basic rate can be applied. That part of the algorithm is meant to prevent recurrences early after termination of atrial arrhythmias by suppressing APBs.

2) Atrial rate stabilization is designed to prevent the onset of atrial tachyarrhythmias caused by atrial premature beats (APBs), followed by pauses and short-long sequences at the atrial level. The APBs trigger temporarily shortening of the pacing interval, which is than gradually lengthened with a programmable increment until the lower 
Rate stabilization pacing availability

during atrial arrhythmia and sinus rhythm

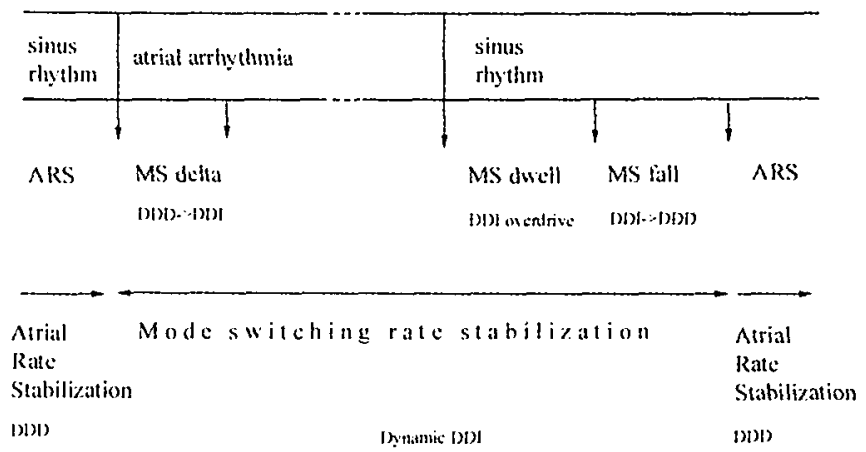

Figure 1. Availability of the dual chamber rate stabilization pacing algorithms in the Jewel AF. During an atrial arrhythmia and immediately following its termination, the mode switching rate stabilization pacing limits increments and decrements in the ventricular rate. In the dynamic DDI mode pacing occurs also in the atrium. Outside mode switching and therapy operations the true atrial rate stabilization pacing limits decrements in the atrial rate. In the DDD mode pacing occurs also in the ventricles.

rate is reached. Atrial Rate Stabilization is available in DDD and AAI mode and provides rate smoothing in the atrium, however in the DDD mode pacing can also occur in the ventricle. Atrial Rate Stabilization is not available during mode switching or therapy operation for ongoing atrial arrhythmia.

\section{Diagnostics}

The diagnostics of the Jewel AF provide in treated atrial arrhythmia episodes information on arrhythmia classification, median PP interval, therapy outcome, registrations of the intracardiac electrogram 5 seconds before the first applied therapy and the marker channel registration before the device declares the end of episode.

\section{Programming settings}

Programming of the dual chamber pacing parameters was done individually in each patient using several steps. During the initial 3 months post implant pacing was programmed in the DDI mode with a basic rate of 40 beats/min and an AV interval allowing spontaneous AV conduction. During that period the occurrence of atrial arrhythmias was monitored while the spontaneous rhythm was allowed as much as possible in the atrium and the ventricle. Using information from the monitoring period the pacing parameters were adjusted, depending on their clinical benefits in the individual patient. During the following 3 months the pacing mode was programmed 
in DDD with mode switching and Atrial Rate Stabilization pacing in 9 patients. In 2 of these patients, in whom a high number $(>3 /$ month) of atrial arrhythmia recurrences could be reduced while using the Atrial Rate Stabilization, this algorithm was programmed on a permanent basis. In 3 other patients with sinus bradycardia following arrhythmia termination Mode Switch Dwell pacing of 70 beats/min was used. In 2 patients the basic rate was increased to 50 beats $/$ min. In 2 patients the pacing mode was programmed after 3 months back in the DDI mode. After the first 6 months following implant, during which the initial adjustments of programming parameters were done, in 7 patients the pacing was in DDD mode and in 8 patients in DDI mode.

\section{RESULTS}

\section{Rate stabilization pacing following atrial arrhythmia termination}

In 600 spontaneously occurring episodes the treated atrial arrhythmia could be documented using the intracardiac electrogram before the first therapy and the marker channel registration following arrhythmia termination. The device diagnosed atrial fibrillation in 341 and atrial tachycardia in 259 cases. Irregularities in atrial rhythm after arrhythmia termination were present in 138 (23\%) of episodes: in 119 cases APBs, in 19 cases nonsustained atrial tachycardias. The APBs occurring immediately after (spontaneous or therapy induced) arrhythmia termination, were still within the device-defined therapy or mode switching operation. The device defines termination of an atrial arrhythmia when 5 consecutive sinus (or atrial paced) beats are present. In patients with frequent APBs it takes long before 5 consecutive sinus beats occur. Only 5 - 15 seconds of sinus rhythm before declaring arrhythmia termination are available from the marker channel registration of episode data. During that time rate stabilization pacing is provided by the mode switching and not the Atrial Rate Stabilization algorithm.

Dual chamber rate stabilization pacing was observed in $245(41 \%)$ of episodes following arrhythmia termination: the Mode Switch Fall was present in 178 cases and the Mode Switching Dwell in 67 cases. Except from episodes with atrial rate irregularities, in 107 (18\%) other episodes rate stabilization functioned as fallback pacing, bringing atrial and ventricular rate down to the lower rate without being interrupted by premature beats. 


\section{Mode switching rate stabilization pacing - effect on atrial rhythm}

Dual chamber rate stabilization pacing after atrial arrhythmia termination was provided in the dynamic DDI mode. This mode takes care of the ventricular rather than the atrial rate regularity, in spite of the fact that sinus rhythm is present again. There are a number of situations where rate stabilization in the dynamic DDI mode functionally resembles atrial rate smoothing (fig 2 ). In the first place, if it functions as fallback pacing. When the spontaneous rhythm is slower than the temporary lower rate of the Mode Switch Fall, than the gradual increment of the pacing interval smoothes the rate at the atrial and the ventricular level at the same time (fig $2 \mathrm{~A}$ ). Furthermore, if the APBs are conducted to the ventricles with a nomal AV time, they result in "prematurity" both in the atrium and the ventricle. In this situation the rate smoothing could be triggered by an atrial or a ventricular premature beat (fig $2 \mathrm{~B}$ ). As soon as atrial and ventricular events are dissociated, the possibilities of the mode switching rate stabilization algorithm to smooth irregularities at the atrial level become limited (fig 3). Ventricular premature beats reschedule the rate stabilization pacing interval, which can be best observed when the intrinsic atrial rhythm is slow. In the presence of a paced atrial rhythm, the appearance of rate smoothing pacing can be triggered by the ventricular premature beat only (fig $3 \mathrm{~A}$ ). In case there are APBs with prolonged or absent AV conduction, the AA intervals are not followed by VV intervals of equal duration. It than becomes evident that rate smoothing respects the ventricular and not the atrial sensed events. The ability to smooth irregularities in the atrial rhythm as well depends on the timing of the APBs in relation to the duration of the post-ventricular atrial refractory period (PVARP) and the basic pacing rate (fig 3B). APBs outside the atrial refractory period inhibit atrial pacing which can result in a longer AV interval. APBs within the atrial refractory period do not inhibit atrial pacing; the sequence of atrial refractory and paced events can lead to iatrogenic atrial bigeminy.

Mode switching rate stabilization pacing can also be observed in treated atrial arrhythmias that terminate spontaneously or become nonsustained (fig 4). When both the atrial and ventricular rate decelerate, the algorithm provides rate smoothing at the atrial and the ventricular level, functionally resembling atrial rate stabilization (fig 4A). On the contrary, in cases where during an atrial arrhythmia a fast ventricular rate is present, attempts to maintain stability lead to a fast pacing rate, sometimes also in the atria (fig 4B). The timing of the atrial paced events can result in an aggressive form of pacing, which might even maintain an atrial arrhythmia that would otherwise become nonsustained or terminate spontaneously. 

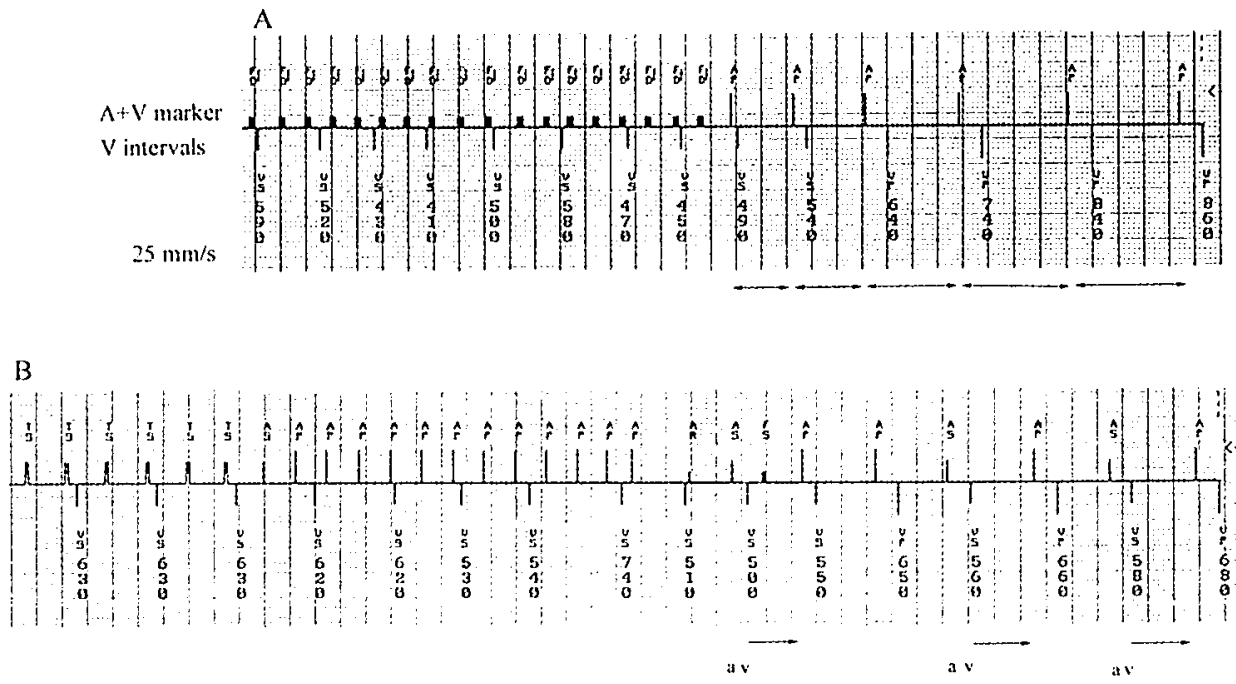

Figure 2. Dual chamber rate stabilization following atrial arrhythmia termination. Mode Switch Fall pacing functions as atrial rate stabilization. In the marker channel registration in both panels the "AS" and the "AP" represent respectively atrial sensed and paced events; the "VS" and the "VP" respectively the ventricular sensed and paced events. A) The left part of the marker registration shows an atrial arrhythmia detected as atrial fibrillation ("FD"), which terminated spontaneously. After termination, the intrinsic atrial rate is slow and there is a paced rhythm present in the atrium and the ventricle. Rate smoothing pacing with increasing intervals $(\leftrightarrow \rightarrow)$ brings the atrial and the ventricular rhythm gradually down to the lower rate. B) The left part of the marker registration shows an atrial arrhythmia detected as atrial tachycardia ("TS") stopped with atrial antitachycardia pacing. After termination, the intrinsic atrial rate approximates the temporary lower rate and there are APBs (a) conducted with normal AV time to the ventricles (AS-VS events). When APBs are normally conducted and the AA interval equals the $V V$ interval, the prematurity at the atrial level is followed by prematurity at the ventricular level $(v)$. Rate stabilization can be due to both atrial and ventricular sensed events. The premature beats cause each time resetting of the temporary pacing interval $(\rightarrow)$. The sequence of a gradual pacing rate decrease until the lower rate is reached cannot be completed, because it is each time interrupted by a new APB, which is conducted to the ventricles. The resulting ventricular sensed events come sooner than the expected ventricular paced events (caused by rate stabilization pacing). This reschedules the rate stabilization pacing interval. 

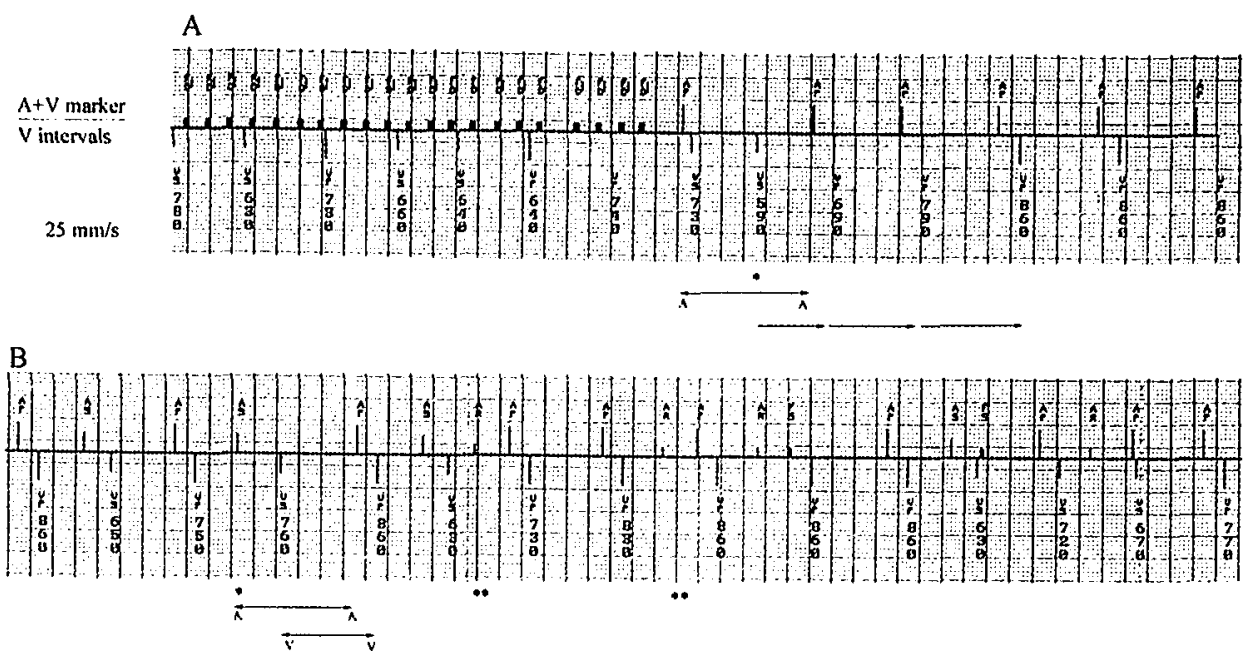

Figure 3. Dual chamber rate stabilization following atrial arrhythmia termination. Lack of rate stabilization pacing in the atrium. Abbreviations in the marker channel as in figure 2. A) The left part of the marker registration shows an atrial arrhythmia detected as atrial fibrillation ("FD") terminating spontaneously. After termination, the intrinsic rate is slow and there is mainly a paced rhythm in the atrium and ventricle. The VPB $\left({ }^{\star}\right)$ triggers rate smoothing pacing $(\rightarrow)$, with the pacing intervals increasing gradually. However, the first pacing interval after that VPB is calculated from the last ventricular and not atrial event. This discloses that rate stabilization concerns the ventricle and not the atrium. The lack of atrial based rate stabilization causes a $1200 \mathrm{msec}$ gap in atrial activity $(\leftrightarrow)$ at the moment of spontaneous arrhythmia termination. B) The marker registration shows rate stabilization pacing some time after the termination of an atrial arrhythmia. The moment of termination is not recorded, probably because it occurred longer before the device could declare the end of the episode. This is the case, when the intrinsic rhythm is interrupted by frequent APBs. The first 4 beats show changes in AV conduction: the fourth atrial beat is conducted with marked PR prolongation $\left.{ }^{*}\right)$. Dual chamber rate stabilization pacing is inhibited at this moment because the rate in the ventricles is faster than the temporary lower rate. This results in the interval between atrial sensed and atrial paced events longer than $1000 \mathrm{msec}(\leftrightarrow)$. The seventh atrial beat, an earlier coupled APB, moves within the PVARP $\left.{ }^{\star \star}\right)$ and being a refractory event it does not inhibit atrial pacing. This can result in an atrial bigeminy pattern. Rate smoothing based on timing of the ventricular and not the atrial events becomes apparent in both $\left({ }^{*}\right)$ and $\left({ }^{* *}\right)$. The timing of atrial paced events is calculated not from the preceding atrial events, but is such, that the timing of their resulting ventricular events allows only limited variations in ventricular rate. Whenever a paced rhythm occurs in the atrium, it is because it is a part of the DDI mode pacing, meant to maintain a stable ventricular rate. 

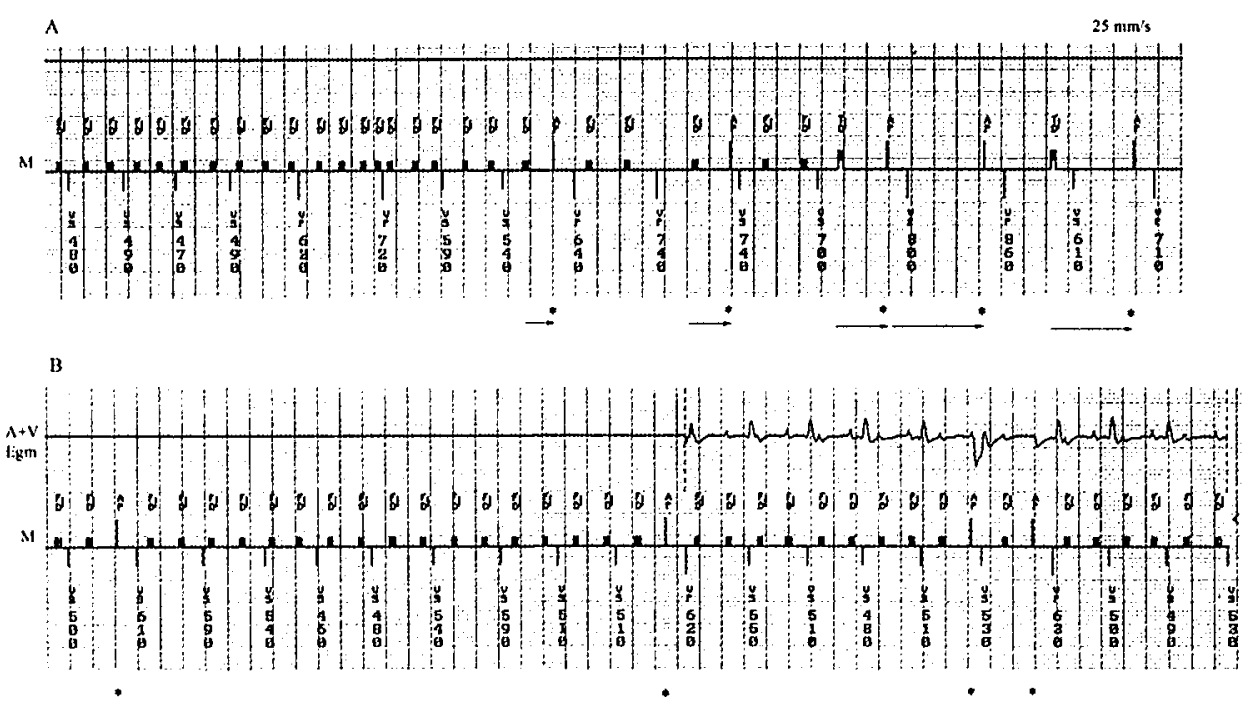

Figure 4. Rate stabilization pacing during an atrial arrhythmia. Abbreviations in the marker channel registration as in figure 2 . A) The left part of the marker registration shows an atrial arrhythmia detected as atrial fibrillation, which decelerates and stops spontaneously. When the median of the atrial rate decreases, atrial premature beats are marked not as fibrillation ("FD") but as tachycardia ("TD") events. Rate stabilization pacing is present during deceleration of the atrial arrhythmia and before spontaneous termination. The intervals between the atrial paced event, the AP $\left({ }^{*}\right)$, occurring as part of rate stabilization pacing, and the preceding atrial arrhythmic event increases gradually. In this case the way of pacing in the atrium $\left(^{\star}\right)$ resembles atrial rate smoothing $(\rightarrow)$. However, it is the occurrence of the ventricular sensed events (VS) that reschedule the timing of the DDI rate stabilization pacing, including the timing of the AP $\left(^{*}\right)$ events. B) The upper strip shows the wideband intracardiac electrogram of an atrial tachycardia that is detected within the atrial fibrillation zone, as it is indicated in the marker channel registration in the lower strip. During the atrial arrhythmia a spontaneous and fast ventricular rate is present. The mode switching rate stabilization pacing attempts to prevent too much variation of the VV intervals. While "stabilizing" the ventricular rate in the DDI mode, it causes pacing in the atrium $\left(^{*}\right)$ at a rate, which approximates the rate of the atrial arrhythmia itself. The fast pacing in the atrium might sustain the arrhythmia. 

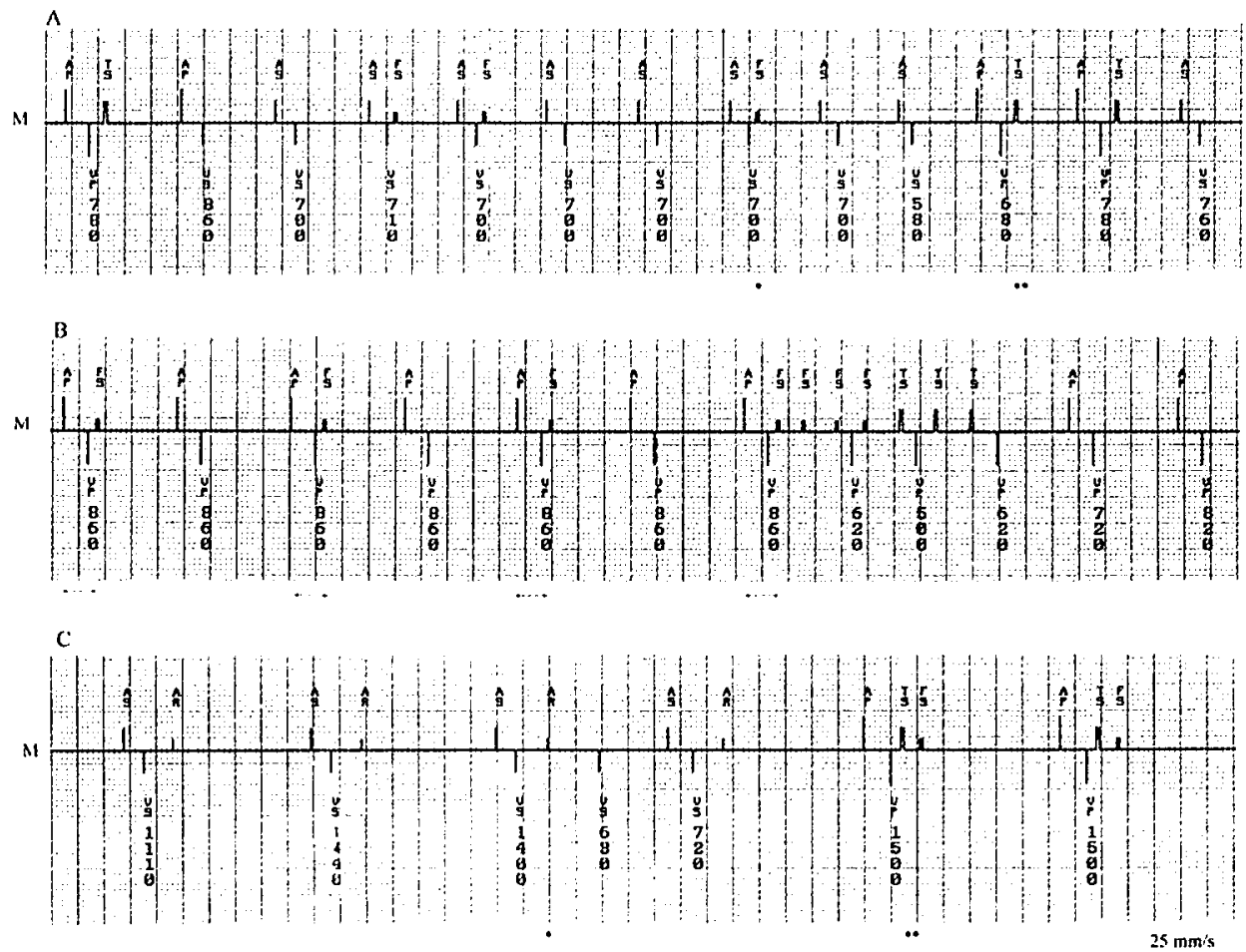

Figure 5. Limitations of the marker channel registration in the evaluation of atrial rhythm following termination of an atrial arrhythmia. An example of an atrial bigeminy pattern. Abbreviations as in figure 2. A) The atrial "TS"(tachycardia) and "FS" (fibrillation) events occurring following both paced and sensed events in the atrium and the ventricle, can represent true atrial bigeminy or far field $R$ wave sensing on the atrial channel. The atrial event after the ventricular spontaneous beat $\left(^{*}\right)$ is a fraction earlier and marked as "FS" when compared with the one after the ventricular paced beat $\left({ }^{\star \star}\right)$, marked as "TS". This suggests far field $R$ wave sensing on the atrial channel. B) Atrial bigeminy can be suspected because the atrial "FS" (fibrillation) event has the same coupling interval $(\leftrightarrow)$ throughout the registration as the "FS", which initiates a nonsustained atrial tachycardia. $\mathrm{C}$ ) Atrial bigeminy can be suspected because the third "AR" (refractory) event having the same coupling interval as the previous two ones, is an atrial conducted beat $\left({ }^{\star}\right)$. The last two beats suggest the presence of respectively both far field $R$ wave sensing and atrial bigeminy $\left(^{* *}\right)$. 
Rhythm registration following atrial arrhythmia termination

The possibility to evaluate the pacemaker function following atrial arrhythmia termination was in some cases limited by the lack of an intracardiac electrogram registration. The marker annotation gave a good idea about the timing but not about the nature of atrial and ventricular sensed events. That information is needed in patients with frequent APBs in whom the permanent use of the Atrial Rate Stabilization is considered. This can also be important in patients with early recurrences after atrial arrhythmia termination. The APB pattern in the marker annotation may represent atrial premature beats, true atrial bigeminy or far field $\mathrm{R}$ wave sensing on the atrial channel (fig 5). The programming measures to cope with the three causes of APB pattern are very different. In case there is true atrial ectopy, permanent programming of the atrial arrhythmia prevention pacing might be considered. In case there is atrial bigeminy, increasing the lower pacing rate might help to eliminate the changes in AA and $R R$ intervals. In case there is far field $R$ wave sensing, decreasing atrial sensitivity usually solves oversensing. Far field $R$ wave sensing at the atrial channel can resemble atrial bigeminy but should not be "treated" with rate stabilization algorithms.

\section{DISCUSSION}

Studies on the prevention of atrial fibrillation by modulating the atrial rate with pacing have reported different outcomes (5-13). Looking at the performance of pacing algorithms by using records of arrhythmia recurrences only, does not elucidate the exact mechanisms of how pacing influences the spontaneous rhythm in different settings. Factors such as algorithm availability, programming settings, synergy between various features of an algorithm and type of arrhythmia may influence the performance of arrhythmia prevention pacing.

Dual chamber rate stabilization pacing in the Jewel AF - two different algorithms

In the Jewel AF the true atrial rate stabilization algorithm, the Atrial Rate Stabilization, is available in the DDD and AAI mode. Since the device has no separately programmable bradycardia and post-shock pacing mode and the ICD patient must be assured of pacing in the ventricle following high voltage therapy, AAI is not an optimal option. Programming the DDD mode in patients with atrial arrhythmias need to be combined with the use of mode switching to avoid ventricular tracking of these arrhythmias. However the mode switching algorithm in the Jewel AF introduces another type of rate stabilization -the mode switching rate stabilization. This pacing 
algorithm operates for a variable period of time also after atrial arrhythmia termination, when sinus rhythm is present again. This means that although the basic pacing mode is DDD and the Atrial Rate Stabilization is used, pacing immediately after atrial arrhythmia termination is provided in the dynamic DDI mode. Considering the device-based definition of atrial arrhythmia termination, frequent APBs may suspend the Atrial Rate Stabilization pacing at a time that the Atrial Rate Stabilization might be useful. The mode switching rate stabilization aimed at smoothing the ventricular rate, causes atrial pacing as part of the algorithm. Depending on the time relation of the underlying rhythm (including premature beats), the temporary lower rate and the programming setting (AV interval, PVARP duration), this pacing algorithm can have different results on the atrial rate regularity. It varies from atrial stimulation that functionally equals Atrial Rate Stabilization pacing, to lack of atrial stimulation, and even introduction of an artificial atrial bigeminy.

The availability of the two rate stabilization algorithms is not only different after atrial arrhythmia termination, but also for atrial refractory sensed events. When the Atrial Rate Stabilization is programmed, atrial rate smoothing is not triggered by the atrial refractory sensed events. These atrial events occurring in the PVARP are most often (in the absence of VA conduction) atrial premature beats. Due to parameter interlocks the PVARP duration is usually long. It means that, if for example the AV time is 190 msec and the PVARP $310 \mathrm{msec}$, the Atrial Rate Stabilization pacing will be triggered by APBs occurring $>500 \mathrm{msec}$ after the $\mathrm{P}$ wave. However, device-defined atrial refractory beats may not truly be physiologically refractory in atrial tissue and also these beats can initiate and reinitiate atrial arrhythmias (14-16). When the mode switching rate stabilization algorithm is used, the opposite situation occurs: atrial beats sensed in PVARP do not inhibit pacing in the atrium. In that case the timing of atrial stimulation in relation to preceding $A B P s$ has less chance to function as rate stabilization in the atrium (fig 3B).

Although the functioning of the dual chamber rate stabilization algorithm was accessible for evaluation only shortly after atrial arrhythmia termination, some observations could be made. The availability of the true atrial rate stabilization pacing in the present device is limited by programmability of pacing parameters. The possibility of a separate bradycardia and post-shock pacing mode and the availability of the Atrial Rate Stabilization in other modes than DDD would support wider and more predictable use of this algorithm. It might be helpful to have the possibility of the dynamic DDI mode in which also the AV interval and the temporary lower rate could change within limits, to improve AV synchrony without permanent DDD pacing. Furthermore, patients in whom permanent DDD pacing is undesirable, could benefit from an automatic change of the pacing mode from DDI to DDD + Atrial Rate Stabilization, after the device has registered an increasing number of atrial arrhythmias in a given period of time. In addition, allowing the atrial rate stabilization for the APBs within (part of) 
PVARP might improve its availability. At present the refractory APBs do not inhibit stimulation in atrium, which in the DDI mode may occur shortly after these beats. In patients with frequent APBs, in whom higher pacing rates while applying rate stabilization are undesirable, availability of beat-to-beat mode switching together with the feature of non-competitive atrial pacing would be helpful. In patients with early reinitiation of atrial arrhythmias following successful termination it might be useful to allow the Atrial Rate Stabilization pacing already during Mode Switch Fall, especially if the duration of this phase of mode switching becomes excessive due to APBs or nonsustained atrial arrhythmias.

\section{Arrhythmia documentation - importance for the clinical functioning of the pacing algorithms}

Clinical aspects of the functioning of rate stabilization pacing may be important in some patients. Mode switching rate stabilization pacing applied when the ventricular rate during atrial arrhythmia is already fast, causes maintenance of that fast ventricular rate (fig 5B). Also Atrial Rate Stabilization applied for frequent and early-coupled $A P B s$ increases the mean (paced) rate at the ventricular level. This may change cardiac function in some patients, for example in patients with coronary artery disease. Furthermore, both rate stabilization algorithms increase the time of pacing in the ventricle in patients who otherwise are not pacemaker-dependent. RV apex pacing may worsen mechanical LV performance short and long term. In our patients without evident clinical advantages of the DDD + Atrial Rate Stabilization algorithm, the permanent pacing mode remained the DDI to allow the spontaneous rhythm. Only in patients in whom a decrease of a high incidence of atrial arrhythmia recurrences during Atrial Rate Stabilization pacing was indicated by device counters, the algorithm was programmed permanently. The lack of an intracardiac electrogram registration of the atrial arrhythmia onset did not allow elucidating the mechanism of a decrease in arrhythmia incidence by Atrial Rate Stabilization in these patients.

The studies on the effect of overdrive atrial pacing on recurrences of atrial arrhythmia do not result in unanimous conclusions. Better outcomes were reported in studies with subsets of patients in whom the mechanism of atrial fibrillation onset was elucidated before applying atrial pacing. The positive effect of suppressing atrial premature beats by higher atrial pacing rates was shown by Coumel (5) in 6 patients with vagally mediated atrial fibrillation. Similarly positive effects of DDD pacing at a rate 10 beats faster than the mean atrial rate was reported by Attuel in 10 patients with sinus node disease (6). In studies with less selected patients the reports do not support pacing as arrhythmia prevention in the general atrial fibrillation population. The Atrial Pacing Periablation for Paroxysmal Atrial Fibrillation Study did not demonstrate any advantage of atrial rate-adaptive pacing in 97 patients known with drug-refractory parox- 
ysmal atrial fibrillation and no symptomatic bradycardia. Although atrial pacing suppressed APB frequency, it failed to reduce atrial fibrillation recurrences (7). In fact atrial fibrillation onset was preceded by atrial pacing in most patients (8). Newer pacing algorithms were developed to avoid continuous high rate atrial pacing and to allow an increase in pacing rate only in the presence of sensed atrial rhythm. The Consistent Atrial Pacing algorithm used in patients with paroxysmal atrial fibrillation increased the percentage of atrial paced rhythm from $65 \%$ to $95 \%$ but could not achieve any significant decrease in atrial fibrillation episodes (9). In this respect the result of the last two studies resemble the results of the CAST study for ventricular arrhythmias: the suppression of APBs is not sufficient to prevent sustained (atrial) arrhythmias. Atrial pacing for prevention of atrial fibrillation was studied also in patients after cardiovascular surgery. In these patients atrial pacing in the first days after surgery was applied to overdrive spontaneous sinus rhythm. Again, the results were encouraging in some studies $(10,11)$ but disappointing in others $(12,13)$. New studies that evaluate the possibility to correct various proarrhythmic factors will give a better idea "when, how and in whom" atrial pacing algorithms are effective (17).

Documenting interactions between the spontaneous rhythm and the pacing algorithms, would give better insight in the functioning of the algorithm in different settings. Clinical performance of the interesting concept of "pacing for arrhythmia" might benefit from adjustments of the way it is being applied.

\section{LIMITATIONS}

The marker channel annotation as the only manner of rhythm registration after arrhythmia termination was in some episodes not sufficient to provide the necessary information on the atrial rhythm. It is uncertain, if mode switching rate stabilization pacing might cause proarrhythmia in some patients or is a less optimal but harmless way of cardiac stimulation.

\section{CONCLUSIONS}

The Jewel AF provides two types of rate stabilization algorithms: the true atrial rate stabilization, the Atrial Rate Stabilization (outside therapy and mode switching operation) and the mode switching rate stabilization (during and immediately after atrial arrhythmia termination). Also when the Atrial Rate Stabilization algorithm is used, pacing following atrial arrhythmia termination is provided in the dynamic DDI mode, in spite of the fact that sinus rhythm is present at that time. The mode switching rate stabilization functioning can be evaluated using rhythm registrations of arrhythmia episodes. This algorithm can only under certain circumstances function as atrial rate 
smoothing as well (sinus bradycardia, APB with normal AV conduction). Stabilizing atrial rhythm at the desired time and manner requires adjustments of the functioning and availability of the Atrial Rate Stabilization algorithm. More diagnostic possibilities are necessary to evaluate the relation between the functioning of the different types of rate stabilization pacing and atrial arrhythmia recurrences, in order to select patients who might benefit from the pacing algorithm as a part of therapy for frequent atrial arrhythmias.

\section{REFERENCES}

1. Saksena S, Mehra R, Ellenbogen K. Pacing for prevention of tachyarrhythmias. In: Ellenbogen K, Kay G, Wilkoff B "Clinical Cardiac Pacing and Defibrillation", 2nd edition, 2000, p 479-496.

2. Delfaut $P$, Saksena S, Prakash A, Krol R. Long-term outcome of patients with drug-refractory atrial flutter and fibrillation and dual-site right atrial pacing for arrhythmia prevention. J Am Coll Cardiol. 1998 Dec; 32(7): 1990-8.

3. Yu WC, Tsai CF, Hsieh MH, Chen CC, Tai CT, Ding YA, Chang MS, Chen SA. Prevention of the initiation of atrial fibrillation: mechanism and efficacy pacing modes. PACE.2000 Mar; 23(3): 373-9.

4. Jewel AF Model 7250 Arrhythmia Management Device system reference guide. Medtronic Inc, 1996.

5. Coumel P, Friocourt P, Mugica J, Attuel P, LeClercqJF. Long-term prevention of vagal atrial arrhythmias by atrial pacing at $90 /$ minute: experience with 6 cases. PACE 1988; 6:552-560.

6. Attuel P, Pellerin D, Mugica J, Coumel P. DDD pacing: an effective treatment modality for recurrent atrial arrhythmias. PACE 1988; 11:1647-1654.

7. Gillis A, Wyse G, Connolly S, Dubuc M, Philippon F, Yee R, Lacombe P, Sarah Rose M, Kerr Ch. Atrial pacing periablation for prevention of paroxysmal atrial fibrillation. Circulation 1999; 99:2553-2558.

8. Hill M, Dinsmmoor D, Klecker K, Gillis A. Impact of atrial pacing on atrial fibrillation onsets in the PA3 trail. Circulation 1998; 17(suppl I):I-711. Abstract.

9. Ricci R, Puglisi A, Azzolini P, Spampinato A, Bellocci F, Padeletti L, Capucci A, Boriani G, Marchini A, Cavaglia S, De Seta F. Consistent atrial pacing algorithm to suppress recurrent paroxysmal atrial fibrillation: a randomized prospective cross over study. PACE 21 (II): 798, 1998.

10. Greenberg MD, Katz NM, Iuliano S, Tempesta B, Solomon A. Atrial pacing for the prevention of atrial fibrillation after cardiovascular surgery. J Am Coll Cardiol. 2000 May; 35(6): 1416-22.

11. Blommaert D, Gonzalez M, Mucumbitsi J, Gurne O, Evrard P, Buche M, Louagie Y, Eucher $\mathrm{Ph}$, Jamart J, Installe E, De Roy L. Effective prevention of atrial fibrillation by continuous atrial overdrive pacing after coronary artery bypass surgery. J Am Coll Cardiol. 2000 May; 35(6): 1411-5.

12. Chung $M$, Augostini $R$, Asher $C$, et al. Ineffectivness and potential proarrhythmia of atrial pacing for atrial fibrillation after coronary artery bypass grafting. Ann Thorac Surg. $2000 \mathrm{Apr}$; 69(4): 1057-63. 
13. Kurz D, Naegeli B, Kuntz M, Genoni M, Niederhauser U, Bertel O. Epicardial biatrial synchronous pacing for prevention of atrial fibrillation after cardiac surgery. PACE 1999; 22:721-726.

14. Sra J, Biehl M, Blanck Z, Dhala A, Jazayeri M, Deshpande S, Akhtar M. Spontaneous reinitiation of atrial fibrillation following transvenous atrial defibrillation. PACE 1998; 21:1105-1110.

15. Timmermans C, Rodriguez L, Smeets J, Wellens H. Immediate reinitiation of atrial fibrillation following internal atrial defibrillation. J Cardiovasc Electrophysiol 1998; 9: 122-128.

16. $Y u W$, Lin $Y$, Tai C, Tsai C, Suing M, Chen C, Hsu T, Ding Y, Chang M, Chen S. Early recurrence of atrial fibrillation after external cardioversion. PACE 1999:22;1614-1619.

17. Anselme F, Saoudi N, Cribier A. Pacing in prevention of atrial fibrillation: The PIPAF studies.J Interv Card Electrophysiol. 2000 Jan; 4 Suppl 1:177-184. 
[162] 


\section{ABSTRACT}

Background: Approximately 25\% of patients receiving an implantable cardioverter defibrillator (ICD) to treat ventricular tachyarrhythmias have documented atrial tachyarrhythmias prior to implant. This study assessed the ability of device-based prevention and termination therapies to reduce the burden of spontaneous atrial tachyarrhythmias.

Methods and Results: Patients with a standard indication for the implantation of an ICD and two episodes of atrial tachyarrhythmias in the preceding year received a dual-chamber ICD (Medtronic 7250 Jewel AF) that utilizes pacing and shock therapies for prevention and/or termination of atrial tachyarrhythmias. In a multi-center trial patients were randomized to three-month periods of atrial therapies "ON" or "OFF" and subsequently crossed over. Analysis was performed on the 52 patients that had atrial tachyarrhythmia episodes and had at least 30 days of follow up with atrial therapies "ON" and "OFF." The atrial therapies resulted in a reduction of atrial tachyarrhythmia burden from a mean of 58.5 hours/month to 7.8 hours/month. A paired analysis (Wilcoxon signed-rank test) showed that the median difference in burden ( 1.1 hours/month) was highly significant $(\mathrm{P}=0.007)$. When a subgroup of 41 patients treated only with atrial pacing therapies was analyzed, the reduction in burden persisted $(P=0.01)$.

Conclusion: In patients with a standard ICD indication and atrial tachyarrhythmias, atrial pacing and shock therapies result in a significant reduction in atrial tachyarrhythmia burden.

\section{Condensed Abstract}

The effect of atrial termination and prevention therapies on atrial tachyarrhythmia burden in patients implanted with a new dual chamber ICD was evaluated. Patients had a standard indication for a ventricular ICD and documented atrial tachyarrhythmias. Patients were randomized to three-month periods during which atrial therapies were "ON" or "OFF" and subsequently crossed over. A paired analysis comparing the "OFF" to the "ON" period in 52 patients showed a significant reduction in atrial tachyarrhythmia burden $(P=0.007)$. Conclusion: Atrial termination and prevention therapies reduce atrial tachyarrhythmia burden in patients implanted with a Jewel AF for a standard ventricular ICD indication. 


\section{INTRODUCTION}

The incidence of paroxysmal atrial fibrillation (AF) in patients receiving an implantable cardioverter defibrillator (ICD) to treat ventricular tachyarrhythmias is about $25 \%$ at the time of the implant (1). Furthermore, ICD patients will develop a first episode of paroxysmal AF after the implant at an annual rate of about $2.6 \%$ (1). Management of atrial tachyarrhythmias in patients with ICDs is important because of the potential for increased morbidity, mortality and the cost of care. The AVID trial showed that AF was an independent predictor of mortality in ICD patients (2), and other studies have shown that atrial fibrillation is associated with an increased mortality in patients with depressed ventricular function $(3,4)$. Additionally, atrial tachyarrhythmias may be symptomatic and may precipitate symptoms in patients with congestive heart failure (5). Sustained atrial tachyarrhythmias may also increase the need for anticoagulation.

Antiarrhythmic drugs have been the mainstay of therapy for the management of patients with AF (6). However, drug therapy is often unsatisfactory due to limited efficacy, risk of proarrhythmia, and undesirable side effects, particularly in patients with depressed ventricular function. In patients who must already be exposed to the procedural risk of ICD placement for ventricular arrhythmias, a dual chamber ICD that offers therapies for both atrial and ventricular tachyarrhythmias may represent a significant advance in arrhythmia management.

A new dual-chamber ICD has been introduced (Medtronic 7250 Jewel® AF) which automatically detects and treats episodes of AF, atrial tachycardia (AT), ventricular fibrillation (VF), ventricular tachycardia (VT), and provides antibradycardia pacing. This device also employs separate algorithms for the prevention of atrial tachyarrhythmias. The primary objective of this study was to evaluate the effect of the combination of prevention and termination therapies on the percent of time an ICD patient spends in atrial tachyarrhythmia (AT/AF burden). A second objective was to determine the effect of these atrial therapies on the frequency of AT/AF episodes.

\section{METHODS}

\section{Study Population and Implant Procedure}

The study was conducted with 269 patients enrolled in 60 centers and followed for 6 months. All patients had a clinical indication for the implantation of a ventricular ICD and two episodes of atrial fibrillation and/or atrial tachycardia in the preceding year with ECG documentation of at least one episode. Patients with chronic fibrillation were excluded from the study. All patients gave written informed consent according 
to a protocol approved by the Human Subjects Committee of the institution at which the devices were implanted. The ICD pulse generator and electrodes were inserted through a single left pectoral incision using standard methods. A 2-lead system was implanted in $84.6 \%$ patients, a 3 -lead system in $14.6 \%$ patients and a 4 -lead system in $0.8 \%$ patients. A step-up atrial DFT protocol was recommended but not required.

\section{Device Characteristics}

A detailed description of the detection and termination algorithms has been reported previously (7). The device discriminates AT from AF based on two programmable detection zones, which may overlap (Figure 1). If the median atrial cycle length is in the overlap zone, the rhythm is classified as AT if it is regular and AF if it is irregular. Outside of the overlap zone, the rhythm is classified as AF if the median cycle length is in the AF zone and AT if it is in the AT zone. Programming of the AT/AF detection intervals was left to the discretion of the physician.

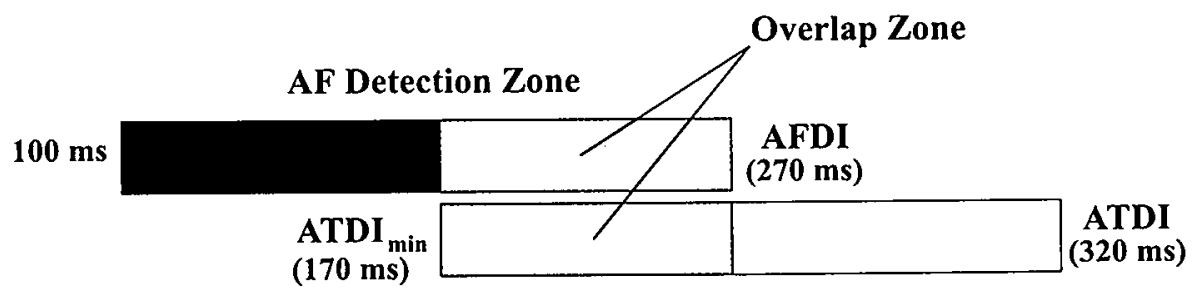

\section{AT Detection Zone}

Figure 1. AT/AF detection zones in the Jewel AF 7250. AFDI $=A F$ detection interval, $A T D I_{\min }=$ minimum AT detection interval, ATDI = AT detection interval. The minimum AF detection interval is $100 \mathrm{~ms}$ and corresponds to the atrial blanking period. The nominal values are shown for the AFDI, the ATDI ${ }_{\min }$, and the ATDI.

The two prevention algorithms and four types of termination therapies that are available in the device are listed in Table 1. For AT episodes, the first 3 programmable therapies are pacing and the last three are shocks. The first two therapies may be programmed to either a Burst+ or Ramp but the third therapy can only be programmed to a $50 \mathrm{~Hz}$ Burst. For AF episodes, the first programmable therapy is pacing $(50 \mathrm{~Hz}$ Burst) followed by a maximum of five shock therapies. For both AT and $\mathrm{AF}$, any of the six possible therapies can be programmed to be skipped. There is a programmable delay ( $0-24 \mathrm{hrs})$ between AT/AF detection and the onset of therapy, programmed separately for pacing (nominal $1 \mathrm{~min}$ ) and shocks (nominal $30 \mathrm{~min}$ ). The nominal programming of the AT pacing therapies is 6 sequences of Ramp (8 pulses), followed by 6 sequences of Burst $+(15$ pulses) followed by 5 sequences of $50 \mathrm{~Hz}$ burst ( $1 \mathrm{~s}$ each). The nominal programming of pacing for the $\mathrm{AF}$ is 10 sequences of $50 \mathrm{~Hz}$ 
burst ( $1 \mathrm{~s}$ each). Programming of all atrial pacing variables, therapy sequences and atrial shocks was left to the discretion of the treating physician.

If atrial shocks were enabled, it was recommended that the first shock for AF be delivered at twice the DFT. All atrial shocks were synchronized to the QRS as sensed by the right ventricular lead, and were withheld if the $R-R$ interval preceding the synchronized QRS complex was less than a programmable value (nominally, $500 \mathrm{msec}$ ), to avoid shock delivery during ventricular repolarization.

Table 1. Atrial Therapies Available in the Jewel AF 7250

\begin{tabular}{ll}
\hline Therapy Type & Function \\
\hline $\begin{array}{l}\text { AT/AF Prevention } \\
\text { Atrial Rate Stabilization }\end{array}$ & $\begin{array}{l}\text { Prevent long pauses following premature atrial complexes by } \\
\text { delivering an atrial pace with an interval equal to the previous one } \\
\text { plus a programmable increment (nominal } 100 \text { msec). } \\
\text { High rate DDI pacing is continued for a programmable duration } \\
\text { immediately following an atrial tachyarrhythmia to overdrive } \\
\text { Suppress ectopy }\end{array}$ \\
Overdrive switchback delay & $\begin{array}{l}\text { Atrial burst delivered at programmable percentage of AT cycle } \\
\text { length followed by two extrastimuli }\end{array}$ \\
Atrial Burst+ & $\begin{array}{l}\text { Atrial autodecremental ramp initiated at programmable percentage } \\
\text { of AT cycle length }\end{array}$ \\
Atrial Ramp & $\begin{array}{l}\text { A train of atrial pacing pulses at 20 msec intervals delivered for } \\
\text { programmable duration (0.5-3 sec) } \\
\text { Atrial shocks delivered over a programmable pathway at }\end{array}$ \\
Atrial Defibrillation & $\begin{array}{l}\text { programmable energies of } 0.4-27 \mathrm{~J} \text { (independent of ventricular } \\
\text { shock programming). }\end{array}$ \\
\hline
\end{tabular}

\section{Study Design}

Ventricular therapies were enabled in all patients. The effect of atrial therapies on AT/AF burden was assessed using a single blind, randomized, crossover design in which atrial prevention and termination therapies were jointly programmed "ON" or "OFF" for 3 months and then each patient was crossed over to the opposite arm for an additional 3 months. Patients were followed with routine device interrogations at 1 month, 3 months, and 6 months post-implant with additional visits as clinically indicated. Changes in antiarrhythmic drugs during the study period were discouraged and use of anticoagulants was left to the physician's discretion. 


\section{Data analysis}

All data beyond the randomization period (i.e., the initial 6 months of follow-up) were censored. To be included in the data analysis, a patient was required to have at least one AT/AF episode during the first 6 months of follow-up and at least 30 days of follow-up in each of the "ON" and "OFF" arms of the study. If patients crossed over early or late, then unequal follow-up times were used in the two arms of the study. Patients were included only if their devices were programmed to deliver both prevention therapies and at least one termination therapy (ATP, $50 \mathrm{~Hz}$ or a shock) in the "ON" arm, and no prevention and termination therapies in the "OFF" arm (i.e. programmed as per protocol). The rhythm (AT or AF) present at the time of first therapy delivery for a given episode was used to classify the episodes, regardless of rhythm transitions (between AT and AF) after initial detection but before therapy delivery. If a shock was delivered at any time during an episode, pacing was considered unsuccessful.

Atrial tachyarrhythmia burden was defined as the total duration of all spontaneous AT/AF episodes divided by the corresponding follow-up time. Atrial tachyarrhythmia frequency was defined as the number of spontaneous AT/AF episodes divided by the time the patient was in sinus rhythm (i.e., follow-up time minus time in AT/AF). The time in sinus rhythm was used to calculate AT/AF frequency, since the prevention algorithms are operative and can reduce the frequency of AT/AF only during sinus rhythm. When patients had more episodes than the device memory could store (30 treated and 128 untreated episodes), the follow-up time in that arm was reduced by the time during which the device memory was saturated. The follow-up time was reduced due to device memory saturation in the "ON" period in 1 patient and 6 patients in the "OFF" period. Paired comparisons of burden and frequency between the "OFF" and "ON" periods were performed using a Wilcoxon signed-rank test since the data did not follow a normal distribution. An analysis was performed to determine if there was a significant order effect, i.e., treating patients in an "ON" then "OFF" sequence vs. an "OFF" then "ON" sequence, and no such effect was seen.

\section{RESULTS}

Characteristics at the time of enrollment for all 269 patients that were randomized in the device clinical trial and for the 52 patients that were the subject of this analysis (discussed below) are summarized in Table 2. The 269 randomized patients had a mean ejection fraction of $34.5 \pm 15.3 \%$, and class II or worse heart failure in $80 \%$. A history of atrial fibrillation was present in $81 \%$ of the patients and atrial flutter in $33 \%$. Despite the requirements of two AT/AF episodes in the year prior to enrollment, an 
Table 2. Patient Demographics

\begin{tabular}{|c|c|c|}
\hline & $\begin{array}{l}\text { Total } \\
(\mathrm{N}=269)\end{array}$ & $\begin{array}{l}\text { Analyzed } \\
(\mathrm{N}=52)\end{array}$ \\
\hline \multicolumn{3}{|l|}{ Gender } \\
\hline Male/Female & $84.7 \% / 15.3 \%$ & $82.4 \% / 17.6 \%$ \\
\hline \multicolumn{3}{|l|}{ Age (years) } \\
\hline Mean \pm SD & $65.3 \pm 10.5$ & $67.7 \pm 10.0$ \\
\hline \multicolumn{3}{|c|}{ Primary Indication for ICD } \\
\hline$S C D$ & $22.4 \%$ & $15.7 \%$ \\
\hline VT & $61.6 \%$ & $70.6 \%$ \\
\hline $\mathrm{SCD} / \mathrm{NT}$ & $13.4 \%$ & $11.8 \%$ \\
\hline Other & $2.6 \%$ & $2.0 \%$ \\
\hline \multicolumn{3}{|c|}{ Primary Cardiovascular History } \\
\hline Coronary artery disease & $67.5 \%$ & $76.5 \%$ \\
\hline Myocardial infarction & $58.4 \%$ & $56.9 \%$ \\
\hline Cardiomyopathy & $47.8 \%$ & $51.0 \%$ \\
\hline Valvular heart disease & $12.7 \%$ & $13.7 \%$ \\
\hline Bradyarrhythmia & $17.2 \%$ & $17.6 \%$ \\
\hline Congestive heart failure & $39.6 \%$ & $52.9 \%$ \\
\hline \multicolumn{3}{|l|}{ Ejection Fraction (\%) } \\
\hline Mean \pm SD & $34.5 \pm 15.3$ & $33.5 \pm 15.7$ \\
\hline \multicolumn{3}{|c|}{ New York Heart Classification } \\
\hline Class I & $17.2 \%$ & $11.8 \%$ \\
\hline Class II & $52.2 \%$ & $64.7 \%$ \\
\hline Class III & $25.4 \%$ & $15.7 \%$ \\
\hline Class IV & $2.2 \%$ & $3.9 \%$ \\
\hline Unknown & $3.0 \%$ & $3.9 \%$ \\
\hline \multicolumn{3}{|c|}{ Atrial Tachyarrhythmia History } \\
\hline $\mathrm{AF}$ & $80.6 \%$ & $86.3 \%$ \\
\hline Atrial flutter & $33.2 \%$ & $43.1 \%$ \\
\hline Other SVT & $6.0 \%$ & $3.9 \%$ \\
\hline \multicolumn{3}{|c|}{ Antiarrhythmic Drug Use at Enrollment } \\
\hline Amiodarone & $31.6 \%$ & $40.4 \%$ \\
\hline Sotalol & $7.4 \%$ & $1.9 \%$ \\
\hline Class I & $9.3 \%$ & $9.6 \%$ \\
\hline Beta-Blocker & $16.0 \%$ & $11.5 \%$ \\
\hline Digoxin & $5.6 \%$ & $1.9 \%$ \\
\hline Ca-Channel Blocker & $5.9 \%$ & $5.8 \%$ \\
\hline
\end{tabular}


atrial episode within the first 6 months of follow-up was observed in only 158 of the 269 subjects (59\%). The devices were programmed with a median atrial tachycardia detection interval of $320 \mathrm{~ms}$ (range, $250-450 \mathrm{~ms}$ ), atrial fibrillation detection interval of $270 \mathrm{~ms}$ (range, $150-300 \mathrm{~ms}$ ), and a minimum atrial tachycardia detection interval of $170 \mathrm{~ms}$ (range, 100-300 ms) (Figure 1).

Of the 269 patients implanted and initially randomized, 52 were included in this analysis. Reasons for exclusion included: failure to crossover ( $n=56)$, non-protocol programming of atrial therapies (e.g. atrial prevention "ON" with therapies "OFF" or other deviation, $n=106)$, lack of at least 30 days follow-up in each arm $(n=7)$, and absence of at least one AT/AF episode during the 6 -months of follow up $(n=48)$. For the 52 properly programmed, randomized, and followed patients, the mean duration of the "ON" and "OFF" arms was $98 \pm 22$ days and $89 \pm 30$ days respectively. In these 52 patients, 44 had at least one atrial pacing therapy with a mean time to initiation of 4 minutes (median, 1 minute; range, 0-60 minutes), and 32 had at least one atrial shock therapy with a mean time to shock of 104 minutes (median, 30 minutes; range, 1-1440 minutes). Atrial tachyarrhythmia burden was significantly reduced with the use of prevention and termination therapies. During the "OFF" period the mean burden was 58.5 hours/month (median, 2.82; range, 0-470), vs. 7.8 hours/month (median, 0.63; range, 0-66) with therapies "ON". This reflects an $89 \%$ reduction in mean burden. The median "OFF"- "ON" difference was 1.1 hours/month $(\mathrm{P}=0.007)$.

Additionally, there was a trend toward a reduction in the episode frequency. The mean episode frequency was 21.6 episodes/month (median, 1.35, range, 0-176) with therapies "OFF", vs. 6.9 episodes/month (median, 1.27; range, 0-52) with therapies "ON" $(\mathrm{P}=0.07)$. Individual patient results are shown in Figure 2. There were 332 AT/AF episodes that were treated in 34 patients. In the 211 episodes treated as AT $(64 \%)$, pacing therapies successfully terminated $62 \%$. In the 121 episodes treated as AF (36\%), pacing therapies successful terminated $24 \%$. The combined pacing success rate for all AT/AF episodes was 49\%. Shock therapies successfully terminated 18/23 AT/AF episodes (78\%).

In addition to the total frequency of AT/AF episodes, we also analyzed the frequency of episodes with durations longer than 20 minutes since the majority of the atrial pacing sequences for termination were completed within 20 minutes. A reduction in the frequency of these episodes provides additional evidence for the contribution of the pacing therapies to burden reduction. The frequency of episodes lasting longer than 20 minutes was reduced from a mean of 3.3 episodes/month (median, 0.5 ; range, $0-30$ ) with therapies "OFF" to 1.2 episodes/month (median 0.3 ; range, $0-14$ ) with therapies "ON" $(\mathrm{P}=0.03)$.

A sub-analysis of burden and frequency was performed in 41 patients in whom no shocks were delivered (i.e. only pacing therapies used). The AT/AF burden reduction 
A

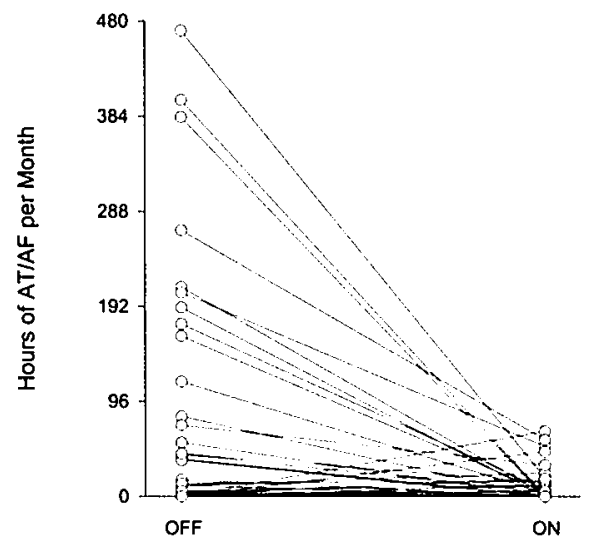

B

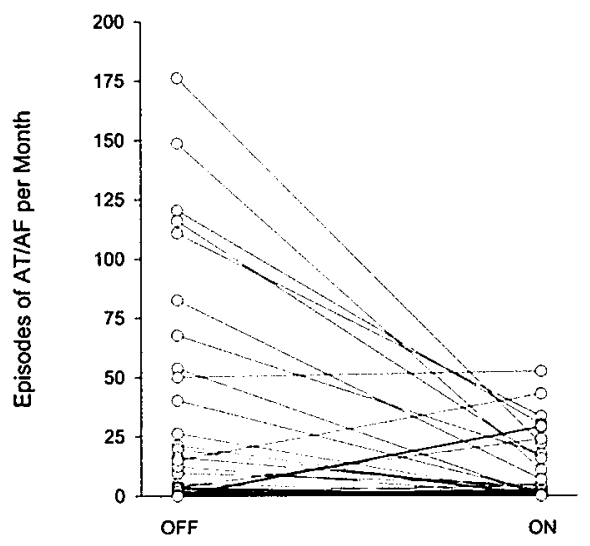

Figure 2. Effect of atrial therapies on AT/AF burden and frequency in 52 patients. (A) Comparison of AT/AF burden in each patient between the periods with atrial therapies " $O N$ " and "OFF". Burden was significantly decreased during the " $O N$ " period ( $P=0.0007)$. (B) Comparison of the frequency of AT/AF episodes in each patient between the periods with atrial therapies "ON" and "OFF". A non-significant reduction in frequency was observed during the "ON" period $(P=0.07)$.

A

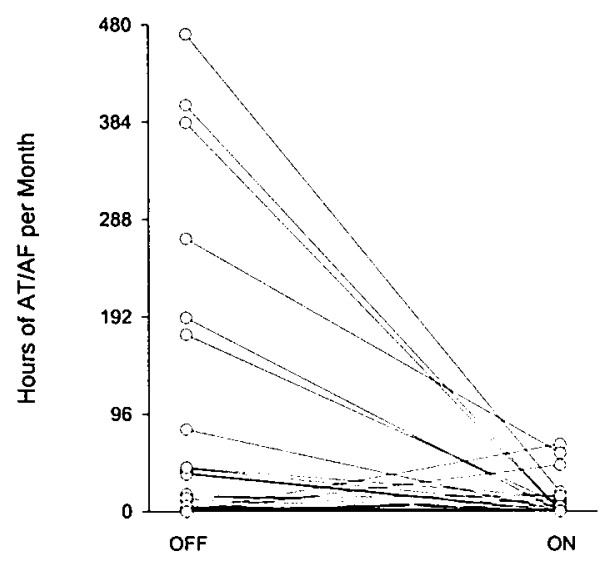

B

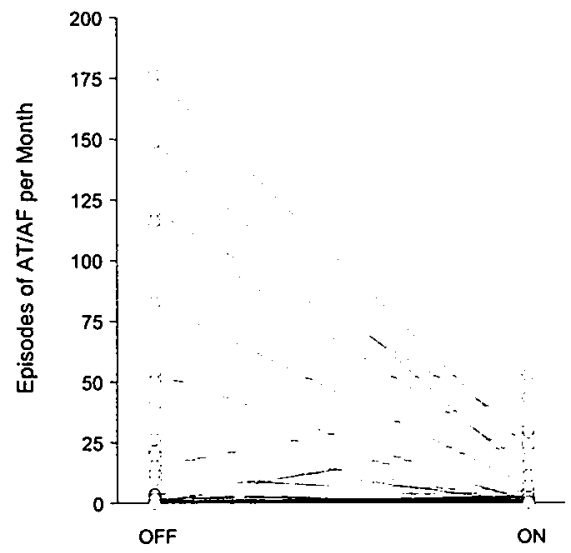

Figure 3. Effect of atrial therapies on AT/AF burden and frequency in 41 patients treated exclusively with pacing therapies. (A) Comparison of AT/AF burden in each patient between the periods with atrial therapies "ON" and "OFF". Burden was significantly decreased during the "ON" period $(\mathrm{P}=0.01)$. (B) Comparison of the frequency of AT/AF episodes in each patient between the periods with atrial therapies " $O \mathrm{~N}$ " and "OFF". A non-significant reduction in frequency was observed during the "ON" period ( $\mathrm{P}=0.16)$. 
persisted in this subgroup treated only with pacing therapies (Figure $3 \mathrm{~A}$ ). During the period with therapies "OFF" the mean AT/AF burden was 53.6 hours/month (median, 2.7 ; range, $0-470$ ) vs. 6.2 hours/month (median, 0.1 ; range, $0-66$ ) with therapies "ON". This reflects an $89 \%$ reduction in mean burden. The median "OFF""ON" difference in burden was 0.54 hours per month $(\mathrm{P}=0.01)$. The corresponding frequency of episodes is shown in Figure $3 \mathrm{~B}$. The mean number of episodes was $22.3 /$ month (median, 1.1; range, 0-176) with therapies "OFF" vs. 6.5 episodes/month (median, 1.0; range, $0-52)$ with therapies "ON" $(\mathrm{P}=0.16)$.

\section{DISCUSSION}

This study found that in patients with a clinical history of atrial fibrillation (81\% of patients) and/or atrial flutter and depressed ventricular function, pacing and shock therapies for termination and prevention of atrial tachyarrhythmias significantly reduced the atrial tachyarrhythmia burden $(\mathrm{P}=0.007)$. Moreover, this burden reduction persisted even when considering patients treated only with painless pacing therapies. The absolute burden reduction was highly variable among patients and tended to be greatest in those patients who had the highest burden during the "OFF" period (Figure 3). Additionally, device-based atrial therapies resulted in a trend toward reduction in the frequency of atrial arrhythmias $(\mathrm{P}=0.07)$. Moreover, there was a significant reduction in the frequency of episodes lasting longer than 20 minutes $(P=0.03)$. Thus, the reduction in burden stemmed from both shorter and fewer episodes. Importantly, the presence of an implanted device permitted accurate determination of arrhythmia burden, independent of symptoms, which has been a limiting factor in other studies that used antiarrhythmic drugs to treat atrial tachyarrhythmias (8).

We could not determine from the present analysis whether the frequency reduction stemmed solely from effective preventative atrial pacing or whether reverse remodeling $(9,10)$ of an adverse atrial electrophysiologic milieu due to prompt arrhythmia termination played a role. Nonetheless, the observed episode reduction may be particularly important as it may enhance tolerability of device therapy for atrial arrhythmias.

\section{Pacing Therapies for the Termination of Atrial Tachyarrhythmias}

The patient discomfort associated with defibrillation has spurred interest in the development of less painful methods to terminate atrial fibrillation. Antitachycardia pacing (ATP) has been highly effective in isthmus-dependent (typical) atrial flutter, which is a highly organized rhythm with a large excitable gap $(11,12)$. Rate adaptive ATP has not been effective in disorganized rhythms, such as atypical flutter or atrial fibrillation 
(13). However, the demonstration that atrial fibrillation has an excitable gap and that high frequency pacing can capture up to a $4 \mathrm{~cm}$ region of the atrium in a fibrillating animal model has provided a rationale for the use of this form of pacing for atrial fibrillation termination (14).

Clinical trials of high frequency $(50 \mathrm{~Hz})$ burst pacing in short cycle length or long-standing atrial fibrillation have been disappointing (15). However, in atypical flutter, which may be a precursor to atrial fibrillation, the results have been more promising, with acute success rates of up to 65 percent (13). In the present study, although $81 \%$ of the patients had atrial fibrillation as their documented clinical arrhythmia, $64 \%$ of spontaneous episodes were initially treated as an atrial tachycardia, suggesting that many episodes of clinical fibrillation begin with a more organized rhythm that subsequently degenerates. The presence of an indwelling device capable of prompt arrhythmia detection may permit termination with pacing techniques during the early, more vulnerable phase of arrhythmogenesis.

Presumably, the use of pacing therapies reduced the need for shocks since pacing therapies were effective in terminating $49 \%$ of all AT/AF episodes. Since the programming of shocks was left to the discretion of the physicians, only 11 of the 52 patients included in the analysis received shocks. Of the 41 patients that received only pacing therapies, the median difference in burden was 0.54 hours/month, with burden lowered with therapies "ON" $(\mathrm{P}=0.01)$. This is an important finding and suggests that a device with pacing and shock therapies may have much more clinical utility compared to a shock-only device.

\section{Role of an Atrial Defibrillator in Patients with Structural Heart Disease}

Previous experience with implantable atrial defibrillators has been limited to patients with structurally normal hearts (16). However, the role for this therapy may be particularly compelling in patients with structural heart disease. Patients with depressed ventricular function and atrial fibrillation have an increased mortality compared to those with normal sinus rhythm independent of the ventricular heart rate-raising the possibility that atrial rhythm control may introduce a mortality benefit $(3,4)$. Additionally, patients with an indication for a ventricular defibrillator must be exposed to the risk of device implantation, so that a device capable of treating both atrial and ventricular arrhythmias may add therapeutic benefit with little or no additional risk. Despite the fact that maintenance of sinus rhythm is more difficult in the setting of cardiac structural abnormalities (17), we found that atrial tachyarrhythmia burden was significantly reduced in this population. The effects of lowering the atrial AT/AF burden on mortality, morbidity and quality of life in ICD recipients needs to be evaluated in future clinical studies. 
Current guidelines recommend the initiation of anticoagulation for atrial fibrillation lasting longer than 24-48 hours prior to cardioversion (18). Prompt treatment of atrial tachyarrhythmias and burden reduction may eliminate the need for anticoagulation in some patients. In this study, $8 \%$ of patients had episodes $>24$ hrs when atrial therapies were enabled while $23 \%$ of patients had episodes $>24$ hrs without atrial therapies. This suggests that the use of the atrial therapies may decrease the need for anticoagulation in this high-risk group of patients.

Atrial tachyarrhythmia prevention has been associated with a reduction in congestive heart failure and hospitalizations in other populations (19). Lowering the arrhythmia burden may reduce the mean ventricular rate, diminish the risk of tachycardia induced pump deterioration, and result in similar clinical benefit in this high risk population, although this was not assessed in the study.

\section{LIMITATIONS OF THIS STUDY}

Implanted patients whose device programming was not in compliance with the study protocol $(n=169)$ were excluded from this analysis. This may have stemmed in part from preliminary data available at the time of the study proposing benefit with atrial therapies. Additionally, another 48 patients had no AT/AF episodes during the 6 months of follow up, and were also excluded. Although this was done since no effect of prevention or termination could be determined in patients with no episodes, due to these exclusions, definitive conclusions about burden reduction must be restricted to a sub-group of 52 patients. Use of antiarrhythmic drugs (amiodarone, sotalol or Class I drugs) was stable in $69 \%$ of the 52 patients during the 6 months of follow-up. Antiarrhythmic drug changes may have contributed to changes in AT/AF burden and/or frequency in some patients.

\section{CONCLUSION}

The use of pacing and shock therapies for atrial tachyarrhythmia prevention and termination reduces arrhythmia burden in patients with a ventricular ICD indication. Atrial arrhythmia burden is reduced due to the presence of shorter and possibly fewer episodes with therapy use. Pacing therapies alone can terminate nearly half of all atrial arrhythmia episodes without the need for shocks. 


\section{REFERENCES}

1. Best PJ, Hayes DL, Stanton MS. The potential usage of dual chamber pacing in patients with implantable cardioverter defibrillators. Pacing \& Clinical Electrophysiology 1999; 22:79-85.

2. The Antiarrhythmics versus Implantable Defibrillators (AVID) Investigators. A comparison of antiarrhythmic drug therapy with implantable defibrillators in patients resuscitted from near-fatal ventricular arrhythmias. New England Journal of Medicine 1997:157601583.

3. Dries DL, Exner DV, Gersh BJ, Domanski MJ, Waclawiw MA, Stevenson LW. Atrial fibrillation is associated with an increased risk for mortality and heart failure progression in patients with asymptomatic and symptomatic left ventricular systolic dysfunction: a retrospective analysis of the SOLVD trials. Studies of Left Ventricular Dysfunction. Journal of the American College of Cardiology 1998; 32:695-703.

4. Deedwania PC, Singh BN, Ellenbogen K, Fisher S, Fletcher R, Singh SN. Spontaneous conversion and maintenance of sinus rhythm by amiodarone in patients with heart failure and atrial fibrillation: observations from the veterans affairs congestive heart failure survival trial of antiarrhythmic therapy (CHF-STAT). The Department of Veterans Affairs CHF-STAT Investigators. Circulation 1998; 98:2574-9.

5. Savelieva I, Camm A. Clinical relevance of silent atrial fibrillation: prevalence, prognosis, quality of life, and management. Journal of Interventional Cardiology Electrophysiology 2000; $4: 369-382$.

6. Prystowsky E, Benson W, Fuster V, et al. Management of patients with atrial fibrillation: a statement for healthcare professionals from the subcommittee on electrocardiography and electrophysiology, American Heart Association. Circulation 1996; 92:1262-1277.

7. Swerdlow C, Schsls W, Dijkman B, et al. Detection of atrial fibrillation and flutter by a dual-chamber implantable cardioverter-defibrillator. Circulation 2000; 101:878-885.

8. Clair W, Wilkinson W, McCarthy E, Page R, Pritchett E. Spontaneous occurrence of symptomatic paroxysmal atrial fibrilation and paroxysmal supraventricular tachycardia in untreated patients. Circulation 1993; 87:1114-22.

9. Tse HF, Lau CP, Yu CM, et al. Effect of the implantable atrial defibrillator on the natural history of atrial fibrillation. Journal of Cardiovascular Electrophysiology 1999; 10:1200-9.

10. Rodriguez LM, Timmermans C, Wellens HJ. Are electrophysiological changes induced by longer lasting atrial fibrillation reversible? :observations using the atrial defibrillator. Circulation 1999; 100:113-6.

11. Friedman PA, Luria D, Fenton AM, et al. Global right atrial mapping in human atrial flutter: The presence of posteromedical (sinus venosa region) functional block and double potentials. A study in biplane fluoroscopy and intracardiac echocardiography. Circulation 2000; 101:1568-1577.

12. Waldo AL, Mackall JA, Biblo LA. Mechanisms and medical management of patients with atrial flutter. Cardiology Clinics 1997; 15:661-76.

13. Giorgberidze I, Saksena S, Mongeon L, et al. Effects of high-frequency atrial pacing in atypical atrial flutter and atrial fibrillation. Journal of Interventional Cardiac Electrophysiology 1997; 1:111-23.

14. Kirchhof C, Chorro F, Scheffer G, et al. Regional entrianment of atrial fibrillation studied by high-resolution mapping in open-chest dogs. Circulation 1993; 88:736-49. 


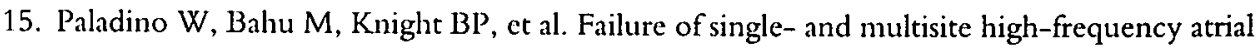
pacing to terminate atrial fibrillation. American Journal of Cardiology 1997; 80:226-7.

16. Wellens HJ, Lau CP, Luderitz B, et al. Atrioverter: an implantable device for the treatment of atrial fibrillation. Circulation 1998; 98:1651-6.

17. Arnar D, Danielsen R. Factors predicting maintanance of sinus rhythm after direct current cardioversion of atrial fibrillation and flutter: a reanlysis with recently acquired data. Cardiology $1996 ; 87: 181-188$.

18. Laupacis A, Albers G, Dalen J, Dunn MI, Jacobson AK, Singer DE. Antithrombotic therapy in atrial fibrillation. Chest 1998; 114:579S-589S.

19. Andersen HR, Nielsen JC, Thomsen PE, et al. Long-term follow-up of patients from a randomised trial of atrial versus ventricular pacing for sick-sinus syndrome. Lancet 1997; 350:1210-6. 
CHAPTER 8

\section{Interactions Between Pacing and} Arrhythmia Detection Algorithms in the Dual Chamber Implantable Cardioverter Defibrillator

Barbara Dijkman MD, Hein J.J. Wellens MD Department of Cardiology

Academic Hospital Maastricht, Maastricht, The Netherlands

Journal of Interventional Cardiac Electrophysiology, in press. 


\begin{abstract}
Dual chamber implantable cardioverter defibrillator (ICD) combines the possibility to detect and treat ventricular and atrial arrhythmias with the possibility of modern heart stimulation techniques. Advanced pacing algorithms together with extended arrhythmia detection capabilities can give rise to new types of device-device interactions. Some of the possible interactions are illustrated by four cases documented in four models of dual chamber ICDs. Functioning of new features in dual chamber devices is influenced by the fact that the pacemaker is not a separate device but a part of the ICD system and that both are being used in a patient with arrhythmia. Programming measures are suggested to optimize use of new pacing algorithms while maintaining correct arrhythmia detection.
\end{abstract}




\section{INTRODUCTION}

In the first generation of the implantable cardioverter defibrillator (ICD) there was no possibility of heart stimulation. With post-shock and anti-bradycardia stimulation pacmaker became an integral part of the ICD systems thereafter. The first pacemakers within the ICD offered only "the basics" of cardiac pacing. Historically, pacemakers as single devices were most of the time more advanced than the pacemaker integrated in the ICD systems. Parameter programmability, antitachycardia pacing, rate response sensors, dual chamber pacing, mode switching and multisite stimulation were sooner available for pacemaker than ICD patients. Therefore in selected ICD recipients who were dependent on (more sophisticated) cardiac stimulation, two separate systems were implanted in some centers. That solution however could lead to device-device interaction (1-7) with potentially dangerous sequels, such as withholding ICD therapy, triggering inappropriate shocks, causing permanent or temporary pacemaker malfunction. Thanks to the currently available technology of pacing integrated in the ICD system those life-threatening interactions can now be avoided. However, also in modern devices the ICD and the pacemaker cannot function independently from each other due to safety of both counterparts of the system. The requirement that detection of ventricular arrhythmia should never be jeopardized by heart stimulation, has consequences for the availability of pacing rates and duration of blanking and refractory periods in a pacemaker. Furthermore, optimal differentiation of various types of ventricular and supraventricular arrhythmia has an effect on sensitivity settings in a pacemaker. In addition, modern dual chamber arrhythmia management devices have new pacing algorithms for atrial and ventricular arrhythmia prevention and rate stabilization that were previously not available in pacemakers. These considerations influence importantly the functioning of an ICD integrated pacemaker.

The following four cases illustrate some of the circumstances in which the functioning of a dual chamber pacemaker is influenced by the fact that it is a part of an ICD system and not a separate device. The "ICD dedicated" way of pacemaker functioning can in turn influence arrhythmia occurrence and detection by ICD. The rhythm registrations were documented either during spontaneous ventricular arrhythmia episodes or "real time" during exercise testing. Only relevant information on device programming is given.

\section{CASE 1}

A 35 years old patient with arrhythmogenic right ventricular dysplasia and episode of ventricular fibrillation (VF) was implanted with the dual chamber 1821 Ventak II DR Guidant model of ICD (8). In this patient periods of sinus bradycardia of $30-40$ beats/min were documented while AV conduction was normal. The Ventak AV has 
the possibility to program separately bradycardia and post-shock pacing mode, which was desirable in this patient. The bradycardia pacing was programmed in AAI 55 beats $/ \mathrm{min}$; the atrial refractory period was $250 \mathrm{msec}$.

Rhythm registration. The surface ECG (fig 1 ) shows during sinus tachycardia occurrence of $A V$ junctional tachycardia and the $P$ waves gradually moving within the $Q R S$ complex (A). Both tachycardias have approximately the same rate (B). When the spontaneous $P$ waves occur late in the AV junctional beats (a) they are conducted to the ventricles with long PR interval (b). Following these conducted beats pacing in the atrium occurs in spite of the spontaneous atrial rate being 110 beats $/ \mathrm{min}$ - far above the programmed lower rate. The paced atrial beats are conducted to the ventricles (c) resulting in ventricular beats 320-360 ms after the preceding QRS complex, sometimes late in the $\mathrm{T}$ wave.

Comments. The real time ICD rhythm registration includes: single lead surface ECG, the atrial intracardiac electrogram (A Egm), the ventricular intracardiac electrogram (V Egm) and the marker channel annotation with interval data (fig 2). It shows the same AV junctional and sinus tachycardias as in fig $1 \mathrm{~B}$. The $P$ waves occur within the QRS complex, mostly early, occasionally late in QRS. When the $P$ waves are simultaneous with the QRS complex $\left(^{\star}\right)$ the atrial activity present in the A Egm is not visible in the marker channel. This is caused by the cross-chamber blanking period at the atrial channel after the ventricular sensed event. When the atrial activity is blanked, it cannot reschedule the basic AAI pacing interval. Stimulation in the atrium occurs when the escape interval, which is calculated from the one atrial event earlier, times out. In this case atrial pacing occurs behind the QRS complexes. The atrial paced beats being conducted to the ventricles give rise to premature ventricular complex occurring just after the $\mathrm{T}$ wave. Not only AV junctional, but also late ventricular beats that occur simultaneously with atrial beats, can cause this pacing pattern.

Background of interaction. In a single chamber AAI pacemaker the basic pacing interval would have been rescheduled by the atrial activity also when it is simultaneous with the ventricular one. In the dual chamber ICD the ventricular sensing remains present during AAI pacing, because of the requirement to detect ventricular arrhythmias. In order to avoid cross-chamber oversensing, the cross-chamber blanking periods are present as a non-programmable feature. In this device the sensing circuit is disabled not only at the ventricular level $(66 \mathrm{msec})$ after atrial paced and sensed events, but also at the atrial level $(86 \mathrm{msec})$ after ventricular paced and sensed events. This results in functioning of the AAI mode that is different in the ICD than in bradycardia devices.

Possible solutions. If the AAI mode remains preferable and this kind of interaction occurs at higher heart rate only during exercise, it might be helpful to program the AAIR mode and to adjust the sensor parameters so, that the sensor driven atrial paced rhythm precedes the AV junctional rhythm. The adjustments should allow spontaneous sinus rhythm as much as possible and the presence of the paced atrial rhythm at 

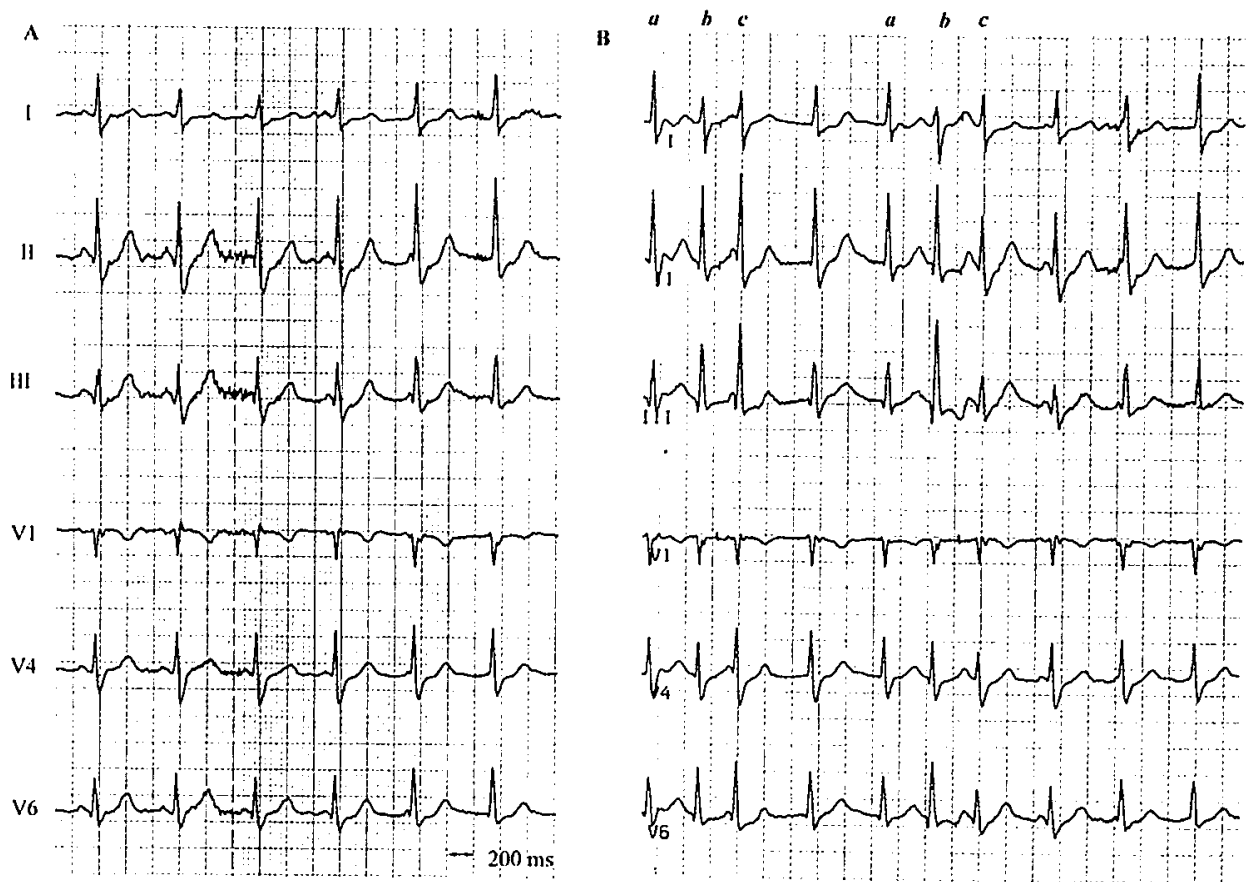

Figure 1. Patient 1. Inappropriate AAI stimulation in the dual chamber ICD. Six simultaneously recorded ECG leads. A) Sinus tachycardia and occurrence of AV junctional tachycardia gradually reaching the same rate as sinus tachycardia. B) Atrial pacing despite high spontaneous atrial rate. The different QRS complexes result from: a) AV junctional beats, b) sinus beats conducted to ventricles with long PR interval, c) paced atrial beats conducted to ventricles.

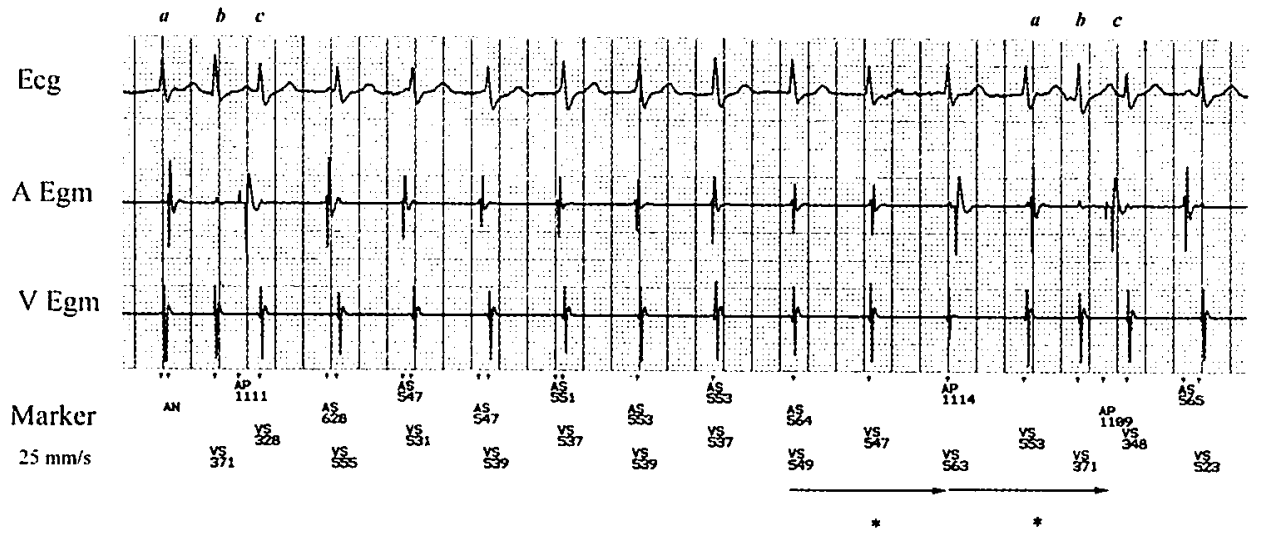

Figure 2. Same patient as in fig 1. ICD registration including single lead ECG, the A Egm, the $V$ Egm and the marker annotation. Dual tachycardia, sinus and AV junctional. Due to the atrial blanking during the ventricular events, present also in AAI mode, some atrial beats do not reschedule pacing escape interval. See text for explanation. 
the heart rates when AV junctional beats appear. It is useful to verify the reproducibility of the heart rate range, when the atrial overdriving is required. If the dual chamber pacing is indicated, the DDDR mode could offer the solution.

If this kind of interaction occurs at higher heart rate not related to exercise it might be helpful to program the basic pacing rate to a lower value. The atrial sensed events that are not blanked have than more chance to reschedule the pacing escape interval. In this patient atrial activity occurred at slightly different moments within the QRS complexes and was not blanked in consecutive beats. If the atrial activity is not blanked in consecutive beats, the AAI lower rate pacing interval can be programmed to the value, which is longer than two PP intervals at the time the interaction occurs. For example, if the lower rate is 40 beats/minute $(1500 \mathrm{msec})$, and the accelerated AV junctional rhythm is faster than 100 beats $/ \mathrm{min}$ (PP interval $<600 \mathrm{msec}$ ), atrial rhythm can still reschedule the pacing escape interval. Even if every second $P$ wave would be blanked (resulting in "PP interval" of $<1200 \mathrm{msec}$ ), the rate of atrial activity that can be sensed is sufficient to inhibit atrial pacing. In case there are consecutive atrial events that fall within the blanking period, this measure would not work.

\section{CASE 2}

A 40 years old patient with an old myocardial infarction and sustained monomorphic ventricular tachycardia (VT) was implanted with the Medtronic $7271 \mathrm{Gem}$ DR model of ICD (9). This patient was not pacemaker-dependent and programming the pacing mode in the DDI 40 beats $/ \mathrm{min}$ with the AV interval of $190 \mathrm{~ms}$ and the duration of the post-ventricular atrial refractory period (PVARP) of $310 \mathrm{~ms}$ allowed the spontaneous rhythm. The duration of not programmable cross-chamber blanking period after paced events is $30 \mathrm{~ms}$ in both atrium and ventricle. The ventricular rate stabilization algorithm was programmed with the minimum ventricular interval $600 \mathrm{~ms}$ and an interval increment of $100 \mathrm{~ms}$.

The Gem DR has a new ventricular rate stabilization (VRS) algorithm designed to prevent the short-long-short sequences started by ventricular premature beats that initiate ventricular arrhythmias in some patients. One of the advantages of the VRS algorithm is, that permanent ventricular stimulation and higher rates are not necessary to avoid pauses in the ventricular rhythm. This is especially of value in patients who are not pacemaker-dependent, in whom rate stabilization pacing is of clinical value, reducing ventricular arrhythmia incidence.

Rhythm registration. The real time ICD rhythm registration shows (fig 3 ) single lead surface ECG, the marker channel annotation and the intracardiac ventricular electrogram (VEgm). During sinus tachycardia of 125 beats/min dual chamber pacing occurs. It is initiated when the antegrade P wave moves within the PVARP becoming 


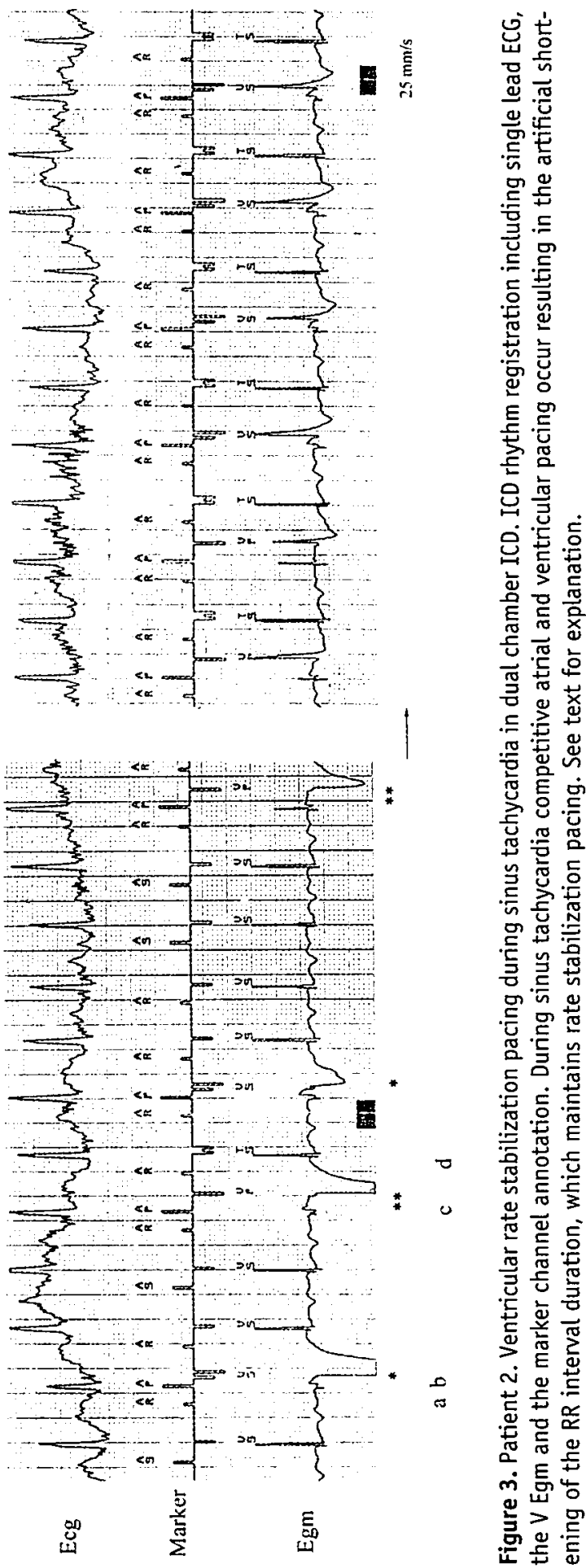


an atrial refractory sensed event (a). The very next atrial paced beat occurs simultaneous with the spontaneous QRS complex sensed within the ventricular safety period (b); therefore the AV interval is shortened $\left(^{\star}\right)$. If the spontaneous QRS complex cannot be sensed because it is exactly within the blanking period of an atrial paced event, it cannot inhibit stimulation in the ventricle (c); stimulation in the ventricle occurs after the AV interval times out $(\star \star)$. Depending critically on the sensing moment of the spontaneous QRS complex in relation to the blanking period of the atrial paced event, either ventricular safety pacing or $T$ wave pacing occurs; in this case fortunately without capture.

Comments. The ventricular paced beats occurring after the spontaneous QRS complexes have number of consequences. First: the moment at which PVARP starts to be calculated shifts in time - it is not started by the spontaneous ventricular event (because these are blanked), but by the ventricular paced event. This makes it even more difficult for the antegrade P wave to move outside the PVARP. Atrial refractory events do not inhibit pacing in the atrium and the summation of both results in competitive atrial pacing. Second: the time interval between the ventricular paced beats and the next spontaneous ventricular event shortens artificially (d). This artificially shorted RR interval becomes the basis for calculating the next rate stabilization interval. This sequence of rate stabilization pacing maintains itself and remains until the stimulation in atrium, as part of dual chamber rate stabilization pacing, ceases. Background of interaction. When the VRS algorithm is enabled, each ventricular event begins a ventricular rate stabilization escape interval (VV) equal to the sum of the previous ventricular interval (RR) and the programmable VRS increment (fig 4). This means that pacing intervals have no fixed value, but are calculated and adjusted for each cycle. At the same time the basic principles of dual chamber timing require that the VV interval is the sum of the AV and VA intervals. To allow the (RR+VRS increment) duration at the ventricular level, pacing in the atrium is scheduled earlier to allow the AV time. Thus, the VA interval equals: (RR+VRS increment)- AV time. In addition, the duration of the AV time is influenced by the rate adaptive $A V$ shortening - feature that mimics the physiologic shortening of the conduction times when the heart rate increases. Although the rate adaptive AV shortening is not available in the DDI mode, it becomes pertinent while the VRS algorithm is enabled. This results in AV intervals that are temporarily shorter than the programmed one, which additionally influences the duration of VA intervals during VRS pacing.

If the programming settings in this particular case are considered, and if we assume that the last $R R$ interval (before the sequence started) is $480 \mathrm{msec}$, the AV interval is 150 msec, and the programmed VSR increment is $100 \mathrm{msec}$, than the VA interval equals $(480+100)-150=430 \mathrm{msec}$. After a VA interval of this duration pacing in the atrium occurs, in this case simultaneously with the spontaneous ventricular event, which results in artificial shortening of $R R$ interval. This shortening of the $R R$ interval 


\section{Ventricular Rate Stabilization}
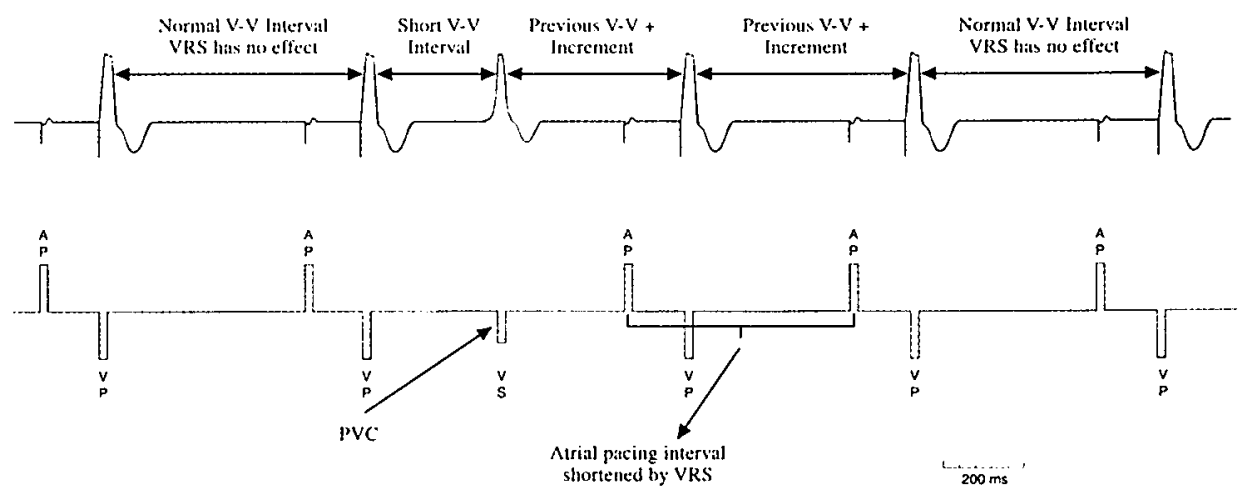

Figure 4. Ventricular rate stabilization algorithm. Each ventricular event begins a pacing interval equal to the sum of the previous ventricular interval and the programmed interval increment. Temporary shortening of the pacing intervals, including the VA time.

causes continuation of the rate stabilization pacing for the longer period of time, than the time rate stabilization would be activated by the spontaneous rhythm only. An "apparent" pacing mediated tachycardia occurs during which the rate stabilization pacing is the cause and the result of competitive atrial and ventricular pacing. Within the timing conditions as in this case, it is only an apparent tachycardia, because the pace pulses do not lead to capture beats.

Possible solutions. Programming measures to prevent this pacing pattern caused by VRS algorithm should be directed towards widening of the detection window for the atrium and the ventricle. If the DDI mode remains desirable, it would be: increasing the minimum rate stabilization interval (for example from 600 to $800 \mathrm{~ms}$ ), shortening of the AV interval, shortening of the PVARP. The first measure, increasing the minimum rate stabilization interval will restrict the application of the VRS pacing during higher spontaneous ventricular rates, as it occurred in this patient. It will still allow using the VRS pacing when the time duration between the VPB and the next ventricular beat is longer than, for example $800 \mathrm{msec}$. The second measure, shortening of the AV interval (and the rate adaptive AV interval) in ventricular timing-based pacing operations postpones the moment of atrial stimulation. When the atrial stimulation is postponed, the spontaneous ventricular activation, gets more chance to appear sooner than the oncoming VRS pacing and to reschedule it. This solution is feasible, if there is only minor difference between the rate of spontaneous rhythm and 
the VRS rate calculated for that moment. The third measure, shortening of the PVARP would reduce the chance for competitive atrial pacing.

It might be necessary to adjust more than one pacing parameters to improve the time relations between the spontaneous rhythm and the VRS pacing.

In addition, the non-competitive atrial pacing feature should truly be available during the ventricular rate stabilization operation. The non-competitive atrial pacing is meant to prevent atrial stimulation shortly after atrial refractory sensed events. When enabled in Gem DR, it delays atrial stimulation scheduled to occur within $300 \mathrm{msec}$ following an atrial refractory event, until this period expires. Delayed atrial stimulation may result in shortening of the programmed AV interval, because the device at the same time attempts to maintain a stable ventricular rate.

The programmability present in the Gem DR allows adjustments of different pacing parameters, while the VRS algorithm can still be used. If the undesirable interactions between bradycardia pacing and tachycardia prevention algorithm remain, despite the available programmability, it might become necessary to disable the algorithm.

\section{CASE 3}

A 67 years patient with an old myocardial infarction was implanted with the 7250 Jewel AF Medtronic ICD (10) because of syncopal VTs as well as paroxysmal atrial flutter and atrial tachycardias. The patient was not pacemaker-dependent and with the pacing mode programmed in DDI 40 beats/min and the AV interval $200 \mathrm{~ms}$, spontaneous rhythm was present.

Jewel AF provides the possibility of detecting and treating ventricular and atrial arrhythmias. The dual chamber arrhythmia detection and discrimination in the device is based on the PR Logic algorithm. This algorithm uses information on rate and pattern of atrial and ventricular events, as well as information on rhythm regularity and AV association, to classify an arrhythmia as ventricular, supraventricular, or dual. The device has two programmable detection zones for supraventricular arrhythmias, the atrial fibrillation (AF) zone and atrial tachycardia (AT) zone, the median PP interval being the arrhythmia classifier. Dual chamber antibradycardia pacing is possible in the DDD mode and in the DDI mode.

$R h y t h m$ registration. Fig 5 shows the snapshots of the real time ICD rhythm registration including a single lead surface ECG, the wideband intracardiac (Atip-Vrig) electrogram and the marker channel annotation. During sinus rhythm beat-to beat far field $R$ wave sensing (FFRS) was present on the atrial channel. During sinus tachycardia (first panel) the following can be observed: 1 ) the summation of both the sinus beats and the FFRS results in an atrial rate which is within an atrial tachycardia detection zone, 2) the ventricular rate enters the detection zone of ventricular tachycardia. At this point the device recognizes correctly the presence of sinus tachycardia (with 


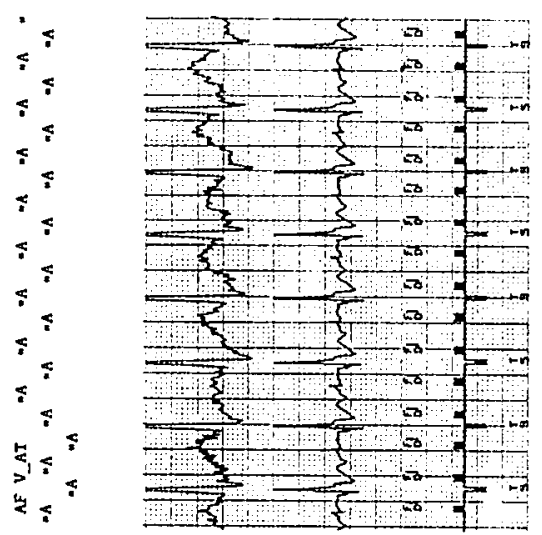

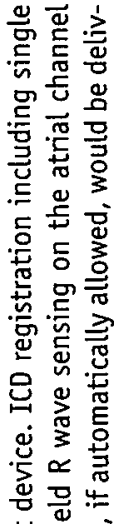

芹究

产市

若范

芒華

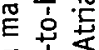

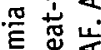

동

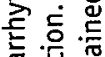

苋

읃

壳 究

气告

空交 会

宁

至

.

등 E

吾荓

冚吉 竞

公究

등

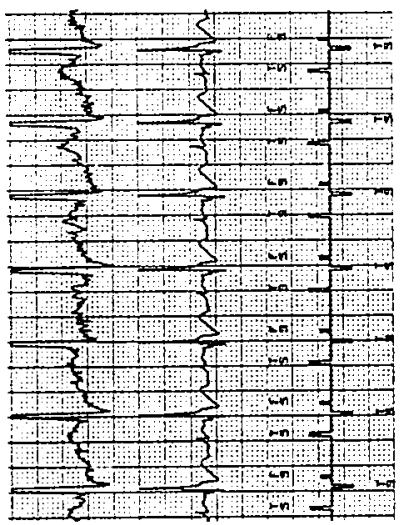

点焉

范

동

흥.:동

过焉

要

묻응 중

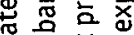

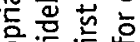

은난

吾范获

空染朵

胥苋

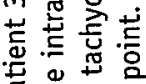

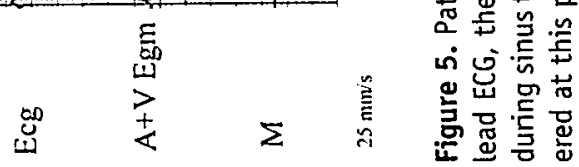


the FFRS) in the VT detection zone. However when the heart rate raises further, atrial tachycardia and at higher rate sustained atrial fibrillation are inappropriately diagnosed (second and third panel). If automatic atrial therapy were allowed, it would be delivered at this point. The discrimination between ventricular and supraventricular arrhythmia remains correct.

Comments. In order to assure reliable atrial arrhythmia diagnosis, also when the ventricular rate is fast, Jewel AF requires wide detection window for atrial events. Therefore the duration of atrial blanking periods in this device is short. The duration of the atrial blanking period after a sensed atrial event is $100 \mathrm{~ms}$, the cross-chamber atrial blanking period after the ventricular pace pulse is $30 \mathrm{~ms}$ and there is no cross-chamber blanking after ventricular sensed events. Short atrial blanking can give rise to FFRS on the atrial channel. By applying the FFRS criterion the device attempts to benefit from the short atrial blanking while not allowing the FFRS to interfere with arrhythmia detection.

Background of interaction. Jewel AF recognizes presence of FFRS during sinus rhythm, when the atrial events show a short-long pattern ( $P$ wave - FFRS $-P$ wave) and the $R P$ interval ( $R$ wave -FFRS) is $<160 \mathrm{msec}$ during at least $10 / 12$ ventricular intervals. In this patient the FFRS criterion is initially fulfilled, but when the short-long pattern of the P wave-FFRS is lost at a higher heart rate, no longer satisfied.

There are two aspects of FFRS in the ICD, which make it different from the same oversensing phenomenon in bradycardia devices: the management possibilities and the consequences. In most dual chamber pacemakers the post-ventricular atrial blanking period is present both after ventricular paced and sensed events. In the Jewel $\mathrm{AF}$ the atrial blanking period is present only after ventricular paced events. To ensure a wide detection window for ventricular and atrial arrhythmias, especially during higher heart rates, there is no cross-chamber blanking following (atrial or ventricular) sensed events. So in pacemakers FFRS can be avoided by adjusting either atrial sensitivity or the duration of the post-ventricular atrial blanking period. In Jewel AF FFRS during the spontaneous rhythm can be prevented only by programming the atrial channel to less sensitive settings. Furthermore, the consequences of erroneous atrial arrhythmia detection are different in bradycardia devices and ICDs. It is false mode switching in a pacemaker but both mode switching and inappropriate atrial therapy in an ICD. The last one can lead to induction of atrial arrhythmia.

Possible solution. If both programming adjustments, the atrial sensitivity and the duration of atrial blanking period are available, the choice between the two measures depends on the atrial arrhythmia cycle length and the amplitude of the intrinsic atrial signal. Atrial arrhythmias, such as atrial flutter (AFL) or AT, having longer cycle length than $\mathrm{AF}$, require wider detection window to be diagnosed by the device. At the same time the amplitude of intrinsic atrial signals during AFL often equals the $P$ wave amplitude during sinus rhythm. In patient with AFL or AT and FFRS, it is better to program 
the atrial channel to less sensitive settings and to keep the atrial blanking period as short as possible. AF having shorter cycle length than AFL is less dependable for detection on the duration of the atrial blanking period. The amplitude of intrinsic atrial signals during $A F$ is often smaller than during sinus rhythm. In patient with AF and FFRS it might be useful to program longer blanking period and to keep the nominal atrial sensitivity settings. In patients with both AF and AFL maintaining a wide detection window remains an important consideration.

\section{Case 4}

A 38 years old patient with sarcoid heart disease and documented VF, syncopal VT and symptomatic VPBs as well as non-sustained atrial arrhythmias was implanted with the Guidant 1810 Ventak AV model of ICD (11). This patient developed gradually AV conduction disturbances and in the end complete AV block; in addition sinus bradycardia was present most of the time.

The Ventak AV has a rate smoothing algorithm that is meant to prevent an undesired sudden decrease or increase in ventricular rate. When enabled it prevents the paced intervals from changing by more than a programmable percentage from one cardiac cycle to the next. Smoothing-up and smoothing-down are independently programmable. This patient was symptomatic both for sudden rate increase due to non-sustained atrial tachycardia and for the pauses following VPBs. The non-sustained atrial tachycardias were most of the time too short to trigger mode switching (for which at least 8 atrial tachycardia beats must be present). The patient associated sudden rate changes with oncoming ventricular tachycardia, which made him anxious.

The pacemaker was programmed in the DDD mode with a lower rate 60 beats $/ \mathrm{min}$, an upper rate 120 beats/min, the $A V$ time of $150 \mathrm{~ms}$ (rate-adaptive shortening possible till $100 \mathrm{msec}$ ), the PVARP was $250 \mathrm{msec}$. Mode switching operation was allowed. Smoothing-up and smoothing-down were programmed with a band of $12 \%$ rate range.

Rhythm registration. Fig 6 shows documentation of spontaneous rhythm with the A Egm, the V Egm and the maker annotations. In $6 \mathrm{~A}$ rate smoothing is illustrated as it was often observed outside arrhythmia episodes in this patient. During dual chamber pacing the ventricular premature beats trigger a temporary increase in stimulation rate, which than gradually decreases $(\rightarrow)$ until the basic rate. In $6 \mathrm{~B}$ the same algorithm operates immediately after the termination of a nonsustained VT. In an attempt to prevent a sudden rate decrease in this case, shorter stimulation intervals are applied $(\rightarrow)$ to smooth down the rate. Dual chamber pacing results in stimulation of the atrium shortly after spontaneous atrial activity. Furthermore after two non-tachycardia beats the VT reinitiates. 

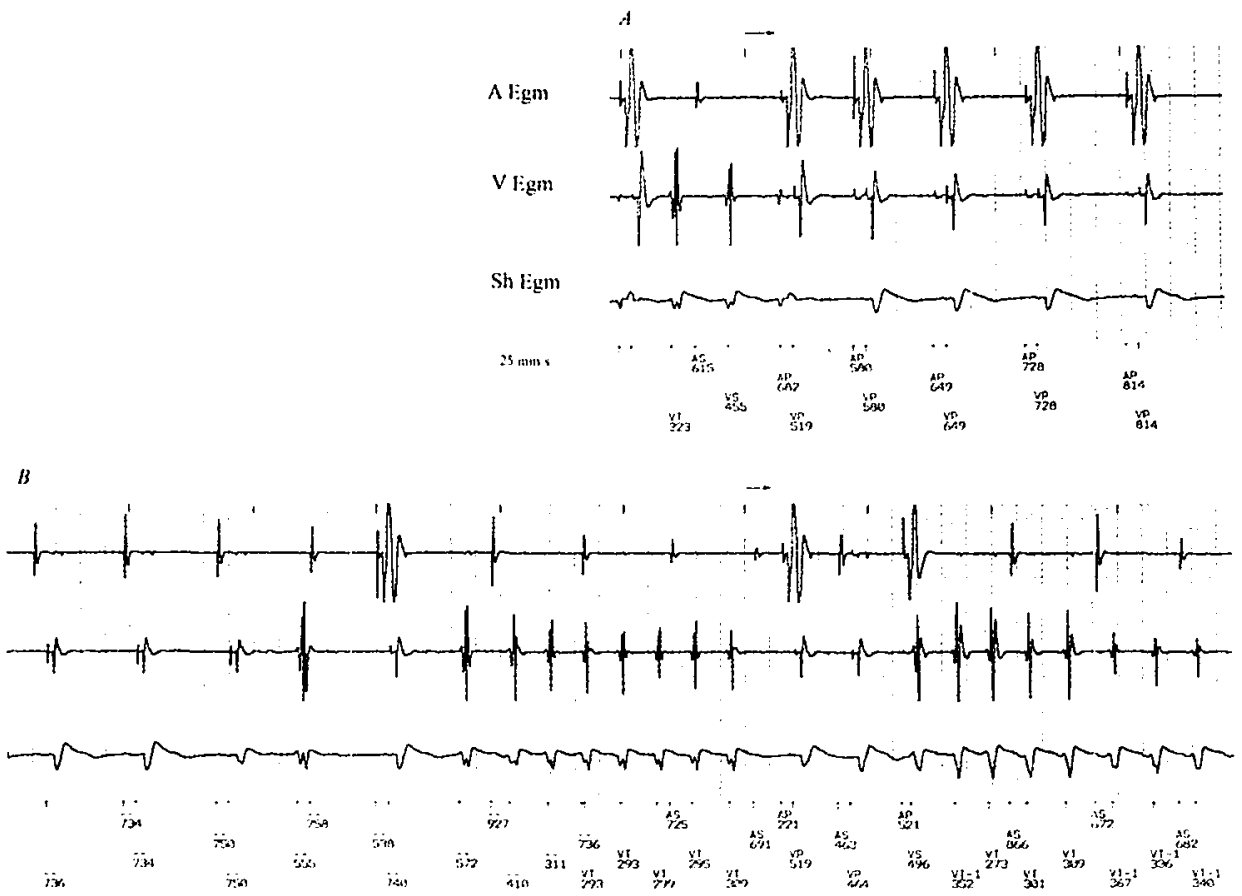

Figure 6. Patient 4. Effect of dual chamber rate smoothing pacing in ICD patient with ventricular arrhythmias. ICD rhythm registration including the $A$ Egm, the $V$ Egm, the shock Egm and the marker annotations. A) Rate smoothing triggered by ventricular premature beats during bradycardia pacing. B) Rate smoothing following ventricular tachycardia termination. Competitive atrial pacing and VT reinitiation. See text for explanation.

Comments. The rate-smoothing feature was applied first in the dual chamber bradycardia devices and later in ICD to control the magnitude of the heart rate variations. Smoothing-down might be helpful in symptomatic patients with sick sinus syndrome, atrial and ventricular premature beats and during upper rate operations. Smoothing-up might be helpful in patients with brief atrial arrhythmias (before mode switching is effectuated) or in case of electromagnetic interference.

Background of interaction. In this patient rate smoothing was effective to alleviate some arrhythmia related complains. Fig 6 A shows such a desirable smoothing-down operation. However, in ICD patients rate smoothing maintains higher ventricular rates also after the termination of ventricular arrhythmias. Although it is uncertain if this was the mechanism in this case, it might reinitiate the arrhythmia. In patients with frequent premature beats the rate smoothing can lead to pacing rates that are continuously higher than the programmed lower (or sensor) rate. In addition, atrial sensed events during a ventricular arrhythmia do not inhibit pacing in the atrium immediately after VT termination, which leads to competitive atrial pacing. 
Possible solution. Programming measures to prevent undesirable high pacing rate in patients with ventricular arrhythmias include: allowing wider range of heart rate changes during rate smoothing (especially "down") and limiting the upper pacing rate in DDD mode. The first measure, the wider range of heart rate changes (for example $25 \%$ ), would allow faster rate decrease following ventricular arrhythmia. Since this might reduce some of the potential benefits of the algorithm in other situations (as shown in fig 6A), it might be useful to have possibility of applying two slopes while smoothing - down the heart rate. This would allow fast rate decrement for the initial 2-3 beats and slower continuation of rate decrease during the remaining beats. The second measure, limiting the upper rate in DDD mode would set the first post tachycardia rate smoothing interval to a longer value. If the continuously high heart rate results from the smoothing being triggered by frequent premature beats it might be necessary to disable the "smoothing down" part of the algorithm.

\section{DISCUSSION}

The device-device interactions in the dual chamber ICDs can be observed also when the tachycardia and bradycardia part of the system are sealed within one can. The interactions are not of mechanical or technical, but of logical nature. The interactions occur mostly during arrhythmia detection and therapy, or outside an arrhythmic episode when the spontaneous heart rate is higher. Under these circumstances the detection window becomes critically dependent on the duration of blanking and refractory periods of both the ICD and the pacemaker. Sensing and pacing related phenomena have very different consequences in both devices - according to the basic concept sensed events inhibit stimulation in a pacemaker but trigger therapy in ICD. Therefore observations from bradycardia devices cannot simply be extrapolated to the ICD integrated pacemaker. Interaction between various pacemaker features and ICD detection algorithms may cause inappropriate pacing that can result in device induced arrhythmias and device mediated arrhythmia; they may also cause inappropriate arrhythmia detection. Although these interactions are usually not life threatening, they can result in electrical ICD therapy or erroneous pacemaker function. The potentially proarrhythmic interactions are not always prevented by parameter interlocks. At the same time interactions may be inapparent during normal sinus rhythm and cannot always be predicted then. Therefore it is desirable to evaluate them under different circumstances, that the ICD patient experiences in normal life, for example at different heart rates, using relevant pacing settings or during an arrhythmia. Exercise testing, induction of ventricular and atrial arrhythmias and Holter registrations, are among the tools to look at the functioning of the algorithms and their interactions. Evaluation of the sensing and pacing related phenomena, is more complete when the 
heart rate becomes higher, because the detection window than become more dependent on the duration of the refractory and blacking periods. In addition, some oversensing phenomena, such as $T$ wave sensing or FFRS, might become apparent only, when the heart rate becomes higher. Furthermore, in some patients the contribution of the paced rhythm increases at higher rates, resulting in additional lengthening of the refractory and blanking periods that are related to the paced rhythm. Increased contribution of the pacemaker rhythm may be due to more outspoken pacemaker dependency; or use of pacemaker features, such as rate response or rate adaptive AV shortening. A Holter registration might be useful to look at the algorithm operation triggered by arrhythmias or rhythm irregularities that cannot be induced or provoked.

In addition to evaluating technical functioning of new algorithms it is equally important to look at their clinical relevance in the individual patient. It might be useful to start that evaluation by allowing patients' spontaneous rhythm, which in most cases can be obtained by programming the pacemaker in DDI mode with a relatively low basic rate. Thereafter, changes in pacing parameters or addition of more complex pacing algorithms can be done, guided by requirements of the clinical situation. The programming endeavors should result in the use of only those features, which offer true clinical advantages to the patient.

Dual chamber ICD technology is an important step forward in management of cardiac arrhythmias. It improves the ability of arrhythmia detection and discrimination, enables physiological pacing, extends ICD therapy with atrial arrhythmia therapy, creates possibility for arrhythmia prevention, allows adequate treatment with beta blocking agents and other antiarrhythmic drugs, helps diminishing arrhythmia related complaints. The advantages of dual chamber devices were achievable thanks to increased device complexity. Although the increased complexity of the ICD detection and pacing algorithms is likely to create situation as described in the four cases, it should never overshadow the advantages that are of importance for the majority of ICD patients. Careful evaluation of new features in individual patients helps to prevent potentially problematic programming settings, while using the best of the advanced ICD technology.

\section{CONCLUSIONS}

The interactions between pacing and arrhythmia management algorithms in the dual chamber ICD may result in inappropriate pacing causing arrhythmia induction as well as in inappropriate arrhythmia detection. Careful evaluation of the functioning of new features remains desirable also when the pacemaker is integrated in the modern dual chamber ICD system. 


\section{REFERENCES}

1. Fogoros RN: Device-device interactions. In: Naccarelli $G$ and Veltri $E$, Eds: Implantable Cardioverter-Defibrillators, Blackwell Scientific Publications, Boston 1993, pp205-215.

2. Singer I, Slater $D$ : Interactions of implantable cardioverter-defibrillators with antiarrhythmic drugs and pacemakers. In: Singer I, Ed: Implantable Cardioverter Defibrillator. Futura Publishing Company, Armonk 1994, pp377-388.

3. Sheahan RG, Dorian P, Poludnikiewicz M, Newman D. Dual device therapy in the setting of changing ICD technology: device-device interaction revisited. PACE 1997; 20:1704-7.

4. Brode SE, Schwartzman D, Callans DJ, Gottlieb CD, Marchlinski FE. ICD-antiarrhythmic drug and ICD-pacemaker interactions. J Cardiovasc Electrophysiol. 1997; 8: 830-42.

5. Geiger MJ, O'Neill P, Sharma A, Skadsen A, Zimerman L, Greenfield RA, Newby KH, Wharton JM, Kent V, Natale A. Interactions between transvenous nonthoracotomy cardioverter defibrillator systems and permanent transvenous endocardial pacemakers. PACE 1997; 20: 624-30.

6. Lambert R, McPherson CA, Lewis RJ, Batsford WP, Rosenfeld LE. Inappropriate sensing of atrial stimuli in patients with third-generation defibrillators and DDD pacemakers. PACE 1998; 21:1225-9.

7. Glikson M, Trusty JM, Grice SK, Hayes DL, Hammill SC, Stanton MS. A stepwise testing protocol for modern implantable cardioverter-defibrillator systems to prevent pacemaker-implantable cardioverter-defibrillator interactions. Am J Cardiol. 1999; 83:360-6.

8. Ventak AV II DR Implantable Cardioverter Defibrillator. Physician's system manual. Cardiac Pacemakers, Inc 1998.

9. Gem DR Implantable Cardioverter Defibrillator. System reference guide. Medtronic USA, Inc 1997.

10. Manual Jewel AF Arrhythmia Management Device. System reference guide. Medtronic USA, Inc 1996.

11. Ventak AV Implantable Cardioverter Defibrillator. Physician's system manual. Cardiac Pacemakers, Inc 1996. 


\section{CHAPER 9}

\section{General Discussion}

Sudden cardiac death is a major health problem throughout the developed countries of the world being estimated as the cause of 250-400 deaths annually in the United States, and the mode of death in about $50 \%$ of all people dying from heart disease (1). Sudden cardiac death is a clinical diagnosis and has a number of causes, rather than being a single and distinct entity. On the basis of series of patients whose rhythm was registered while dying suddenly, it was stated that $80-90 \%$ of sudden cardiac death is due to ventricular arrhythmias (2). The implantable cardioverter defibrillator (ICD) was developed to treat the specific cause of sudden arrhythmic cardiac death, namely ventricular fibrillation (VF) or haemodynamic unstable ventricular tachycardia (VT). From the first series of cardiac arrest survivors implanted with the ICD until now, evidence has grown confirming the superiority of ICD therapy compared to other treatment modalities in patients with life threatening ventricular arrhythmias. ICD therapy resulted in a significant reduction (20-30\%) of not only arrhythmic but also overall mortality, as proven by retrospective and prospective studies (3-7).

The first generation ICDs fulfilled their primary task of being capable of detecting and interrupting fast ventricular arrhythmias. However, more capabilities turned out to be desirable for successful and complete arrhythmia therapy in patients with various structural and functional heart disease. Soon, the next two generations ICD provided programmable detection zones, different electrical therapies (antitachycardia pacing (ATP), low energy cardioversion and defibrillation), bradycardia and postshock back-up pacing, possibilities of non-invasive electrophysiological examination and arrhythmia diagnostics. Sophistication increased in subsequent models of devices. This development of ICD therapy has been described under "ICD technology development and current status".

In 1995 a new generation dual chamber ICDs was introduced, in which the new capabilities were not simply the extension of existing ones. The addition of information from the atrial channel allowed, for the first time the diagnosis and evaluation of all cardiac arrhythmias and their mutual relationship, the ultimate aim being to better treat and prevent those arrhythmias. Furthermore, it became possible to pace and 
defibrillate the atrium. The possibilities and limitations of dual chamber arrhythmia management in those new devices were evaluated in this thesis.

Correct arrhythmia detection is a fundamental requirement for adequate ICD therapy. Since the first models of ICD many technological endeavors were made to improve device detection abilities. In the first devices the primary aim was to ensure safe recognition of life threatening ventricular tachyarrhythmias. Later attention was directed to discriminate fast (haemodynamically unstable) from slower (haemodynamically stable) ventricular tachycardia allowing the use of different therapeutic modalities. The next step was an attempt to differentiate between ventricular and supraventricular tachyarrhythmias to avoid inappropriate therapies. Technological tools that enabled these detection improvements included: programmability of tachycardia detection rate and duration, number of detection zones, detection enhancements such as sudden onset and rate stability, QRS width and morphology templates analysis. Presently available single chamber detection algorithms allow $90-100 \%$ sensitivity and $46-98 \%$ specificity of the ventricular tachyarrhythmia therapy (8-12). In chapter 3 functioning of the new dual chamber detection algorithm, the PR Logic, was studied in 1367 spontaneously occurring arrhythmias in 35 patients implanted with the first two models of dual chamber Medtronic ICDs (Jewel $A F$ and Gem DR). If the accuracy of ventricular arrhythmia detection is looked at in the way it is mostly done in the literature, $100 \%$ sensitivity and $96 \%$ specificity was found. In our study a different approach was chosen. The performance of the PR Logic was evaluated in a subgroup of 310 diagnostically difficult arrhythmias, in which tachycardia detection occurred both at the atrial and the ventricular level and the VT: SVT discrimination rules were implemented before the tachycardia could be classified. These were double (atrial and ventricular) tachycardias and supraventricular arrhythmias occurring in the VT/VF zones.

Studies evaluating the performance of detection enhancements include mostly all recurrent ventricular arrhythmias, also those in which the ventricular rate criterion only is sufficient for correct arrhythmia classification and the detection enhancements are not challenged. The approach in those studies leads to optimistic results, considering that in our study only $23 \%$ of spontaneous arrhythmias required VT: SVT discrimination rules for proper diagnosis and these arrhythmias were the object of evaluation. Furthermore, there was no rate limit used in assessing the tachycardia, whereas in other studies supraventricular arrhythmias with fast ventricular rates, for example $>160$ beats/minute, were excluded because it was not possible to program detection enhancements for higher ventricular rates. In addition, we also analyzed arrhythmia episodes in which the PR Logic showed the expected behavior, but the result was inappropriate therapy, for example on redetection. We considered expected algorithm limitations as being undesirable, negotiable, and clinically rele- 
vant. Moreover, in our study arrhythmia classification by the ICD was verified in the intracardiac atrial and ventricular electrograms in all arrhythmia episodes. That validation of device diagnosis is important: device-based arrhythmia classification is from the viewpoint of the device always correct. After all the differences in study design were considered, we found that in $100 \%$ of double tachycardias the VT was correctly diagnosed and received priority of treatment and that the supraventricular tachyarrhythmia (SVT) in the VT zone were misdiagnosed in $19 \%$ of episodes as VT. The causes of inappropriate discrimination were analyzed and programming measures to improve detection specificity using current algorithms (feasible in only $22 \%$ of failures) discussed. Considering that both functioning of the PR Logic and parameter settings in the individual patient were of influence on arrhythmia detection and discrimination, our study has implications for both design of future detection algorithms in the ICD and programming measures by the physician.

The design of the detection algorithm should provide more programmability. There are only two programmable elements in the present PR Logic: the type of SVT for which VT: SVT rejection rules are applied, and the SVT Limit. However most of the criteria used for the dual chamber arrhythmia discrimination are not programmable as such: definition of the SVT, ventricular cycle length regularity, AV association, far field $R$ wave sensing (FFRS) criterion, and the AV pattern recognition.

The $A V$ pattern recognition divides the $R R$ interval into four zones to accommodate the expected atrial event: anterograde $P$ wave, retrograde $P$ wave, FFRS, atrial fibrillation and flutter (AF/AFL) sensing. This means that only atrial tachyarrhythmias, which are conform to this expectation can appropriately be detected. Since sinus and atrial tachycardias with 1:1 AV conduction and $\mathrm{PR}>\mathrm{RP}$ zone were responsible for the majority of diagnostic mistakes and maladaptation of the $P R$ interval to higher heart rate was often seen in our patients, it is important that the zones for the anterograde and retrograde $\mathrm{P}$ become programmable. In patients with absent VA conduction and prolonged $P R$ interval it might be rational not to reserve a zone for the retrograde $P$. In patients with intermittent VA conduction, a programmable ventricular rate limit within which the anterograde and retrograde zones are defined would be useful.

The FFRS criterion as currently defined has not much chance to be fulfilled, because in most patients it whom the phenomenon was observed, it was intermittently present. The best solution for FFRS would be improved processing of atrial signals so, that this cross-chamber oversensing will not appear at all. Until that time the criterion should be programmable as well.

The SVT discrimination limit which is programmable as low as $240 \mathrm{msec}$ is already liberal and with few exceptions sufficient to allow almost all episodes of AF/AFL to be recognized as SVT. In view of the questionable reliability of the elements of the PR Logic (such as PR: RP ratio and RR rate variability) at that short tachycardia cycle 
length, as well as the potential consequences of diagnostic mistakes, setting the minimum SVT discrimination limit at that value seems justified.

The possibility of arrhythmia discrimination should be present also on redetection, since delivering inappropriate therapy of increasing aggressiveness is not always safe. Sequences of ICD therapies when a shock for VT induces AF with fast ventricular rate, leading to inappropriate shock (because there is no VT: SVT discrimination on redetection), causing VF, etc., may end up with VT or VF for which no therapy is available (13).

In difficult arrhythmias that remain a diagnostic problem for PR Logic, it would be useful to add some of the single chamber detection enhancements, like QRS morphology analysis.

Automatic measurement and monitoring of some parameters (for example VA conduction, FFRS, QRS morphology during sinus rhythm and tachycardia) by the ICD might be helpful to optimize the detection algorithm.

Information on patients' arrhythmia before ICD implantation is important for the choice of device, since the algorithms for arrhythmia detection and discrimination differ among different ICDs. It is important to be informed about the possibility of ventricular rate overlap during VT and sinus tachycardia on exercise, especially in patients in whom the VT cycle length is long. Furthermore, about the presence and type of atrial arrhythmias and their ventricular rate. Moreover, about the AV and VA conduction under different circumstances. In addition, pacemaker dependency especially at higher rates, will be of influence on arrhythmia detection window. A complex arrhythmia history with coexistence of different types of tachyarrhythmias at the ventricular and supraventricular level, as well as bradycardia, is something not every dual chamber ICD model is capable to cope with at this time.

Careful evaluation of the arrhythmia detection and discrimination algorithms in ICD during induction study on predischarge- and during exercise testing together with the information on arrhythmia history before implant, allow to design initial device programming. Observations on detection of spontaneous arrhythmia recurrences allow further adjustments. Shortcomings of the detection algorithm can in many cases be corrected by programming measures. The consequences of each element of the PR Logic are not the same among different patients, as is illustrated in the ICD rhythm registration in chapter 3 . For example, in a patient experiencing double tachycardia, VT and AF, RR interval regularity during the VT is important for the detection time of the VT. In VTs showing "normal" $R R$ variation ( $30-40 \mathrm{msec})$ the detection time, if nominally programmed, might become excessively long. Another example, in a patient with intermittent FFRS, counting on the automatic FFRS criterion is insufficient, because intermittent FFRS during sinus tachycardia will lead to inappropriate $\mathrm{VT}$ detection. The only way to allow sinus tachycardia to be properly discriminated is to adjust the sensitivity of the atrial channel. On the other hand making the atrial 
channel less sensitive might negatively influence the detection of atrial arrhythmias. To deal with this dilemma, the amplitude of the FFRS and its behavior during exercise need to be evaluated and the reliability of atrial arrhythmia detection verified during an induction study.

In some cases the programming measures to help the PR Logic discriminating arrhythmia will be not possible because of the lack of algorithm programmability for a certain parameter. It is important to realize that in those cases the dual chamber ICD will function as a single chamber device in respect to that feature. The approach is than reduced to the approach known from the era of single chamber ICDs: influencing ventricular rate during ventricular and supraventricular arrhythmias, for which pharmacological interventions are necessary; programming longer detection times, although this is not without consequences; limiting patients' exercise. All those measures meant restrictions for the patients and in practice could not be relied on for arrhythmia discrimination.

In summary, our study on dual chamber arrhythmia detection looks more specifically at the performance of the detection algorithm in diagnostically difficult arrhythmias, than other studies. Not only device defined, but also clinically relevant PR Logic performance was evaluated. The PR Logic allows safe detection of ventricular arrhythmias but requires further extension and programmability to improve VT: SVT discrimination rules. The importance of programming measures in different patients with a complex arrhythmia history is illustrated for different elements of the PR Logic.

Technology of the dual chamber ICD was driven by two impulses: the DDD pacing possibilities and improved detection specificity to avoid inappropriate therapy for supraventricular arrhythmias. The performance of the dual chamber ICDs has so far been judged based on the performance of these two features (14). However, no studies addressed the question, whether the ventricular arrhythmia itself, being the primary aim of the ICD therapy, could positively be influenced by the dual chamber device other than withholding inappropriate shocks. In chapter 4 use of information from the atrial channel to guide antiarrhythmic and electrical therapy for ventricular arrhythmias is reported analyzing 724 spontaneous ventricular arrhythmia episodes occurring in 41 patients implanted with dual chamber ICDs (Guidant and Medtronic). The study resulted in a number of interesting findings.

Studies on initiation of ventricular arrhythmias during supraventricular arrhythmias concentrated mainly on AF and the presence of short-long-short sequences (15-19). We found that the most common supraventricular tachycardia preceding the onset of VT is sinus tachycardia. This was in spite of the use of beta-blocking agents in $90 \%$ of our patients and in spite of the absence of ventricular arrhythmias during exercise testing. Differently from VTs occurring during exercise testing in hospital, it was not easy to suppress VTs occurring during sinus tachycardia in the ambulatory patient by 
increasing beta-blocker dosage. Some of the patients do not recall physical exercise when VT occurred during sinus tachycardia. Therefore other causes of sinus tachycardia than physical exercise may be suspected, such as mental stress or autonomic influences (20). Furthermore, we found sinus tachycardia developing during $23 \%$ of ventricular arrhythmias. Different from previous reports $(21,22)$, sinus tachycardia developing during VT occurred in ICD patients without any signs of haemodynamic instability. VTs also having sinus tachycardia responded poorly to previously successful antitachycardia pacing. In these patients discontinuation of amiodarone and change of the betablocking agent to propranolol resulted in easier pace-termination of VTs.

Atrial arrhythmias were present at the time of ventricular arrhythmias in $7 \%$ of documented ventricular episodes. In a subgroup of patients known with an atrial arrhythmia before ICD implantation, $21 \%$ of VT episodes were preceded by atrial arrhythmia, but only $0.9 \%$ of documented ongoing atrial arrhythmias became double tachycardia. The onset of atrial tachycardia was immediately followed by the onset of VT even more sporadically (only 3 cases). VT initiation during an atrial arrhythmia was preceded by a fast ventricular rate in $58 \%$ cases and by a short-long-short sequence in only $10 \%$ of cases. In the remaining cases the ventricular rate was neither fast nor irregular. Furthermore, we observed atrial arrhythmias arising during fast VT with and without VA conduction.

Dual chamber electrogram (Egm) documentation of recurrent ventricular arrhythmias revealed that in $8 \%$ of cases the VT cycle length was at the edge or outside the programmed detection zone. This was particularly the case in patients with dilated cardiomyopathy and arrhythmogenic right ventricular dysplasia. Dual chamber Egm documentation was very valuable to evaluate arrhythmia detection and redetection especially when the VT cycle length and QRS morphology altered during one episode. Presence of multiple VTs within one detected episode might be inapparent in a single chamber V Egm. Changes in morphology of a single chamber V Egm and sudden rate deceleration after ventricular therapy may erroneously suggest VT termination. It is the A Egm, which allowed information about VA dissociation and the sinus rate. This implicates that in studies comparing dual and single chamber detection algorithms, limitations of self-assessment capabilities in single chamber ICDs should be considered.

In summary, the diagnostic possibilities in the dual chamber ICD allow better insight in the mechanisms contributing to onset and maintenance of ventricular arrhythmias. The currently available dual chamber diagnostics helped in some patients to influence positively ventricular arrhythmia occurrence and its therapy. The study stresses the value of betablocking agents in patients with ventricular (and atrial) arrhythmias treated with ICD and suggests that the dose of beta-blocker required to achieve an antiarrhythmic effect might be higher than to achieve other cardiovascular effects. 
The incidence of paroxysmal AF in ICD recipients is about $25 \%$ at the time of implant and the annual rate of developing a first paroxysmal AF episode is about 2,6\% (23). Atrial arrhythmias in ICD patients are important because these arrhythmias are an independent predictor for increased mortality and morbidity (24). Dual chamber ICD technology created the possibility to detect and treat atrial tachyarrhythmias with atrial fibrillation as the most important one, and the Medtronic Jewel AF was the first Arrhythmia Management Device model to have that possibility.

Using the diagnostics of the Jewel AF allowed us to study (chapter 5) atrial arrhythmias occurring under ambulatory conditions in 30 patients with structural heart disease. These patients had documented ventricular and atrial arrhythmias (AF being present in 27 patients), with most of them on a beta-blocking agent and a class III drug. This is the first study, in which the analysis of the intracardiac electrogram and marker registration was added to the rate criterion in order to achieve a more specific classification of the arrhythmia and therapy outcomes. Furthermore, in this study atrial arrhythmias were documented and treated early after onset. During the follow-up period of 20 (2-35) months atrial arrhythmia recurrences occurred in $50 \%$ of patients, all known with AF before implant. The baseline characteristics of patients with and without atrial arrhythmia recurrences were similar with regard to known "risk factors" such as an enlarged left atrium, presence of important mitral valve dysfunction or diminished left ventricular function. Different were the history of persistent $\mathrm{AF}$ before implant and worse functional class in patients with recurrences. Therefore in this group of patients, atrial arrhythmia documentation based on symptoms does not represent their true arrhythmia incidence and cannot be used to judge efficacy of, both device and pharmacological therapies. This is relevant for patient eligibility in studies on atrial arrhythmia treatment (therapy or prevention) using device therapy, in which not the ventricular arrhythmias, but atrial arrhythmias are going to be the reason for device implantation.

The most frequently recurring atrial arrhythmias had at their onset a higher degree of organization and a longer cycle length than AF. We diagnosed $19 \%$ episodes of AF, $20 \%$ fast polymorphic ATs, $57 \%$ fast monomorphic ATs and $4 \%$ slow AT. When device- based arrhythmia classification was applied $55 \%$ of arrhythmias were classified as AF. This is important for the interpretation of other studies on the clinical results of the Jewel AF. In other studies device-based arrhythmia classification was used, using mainly nominal programming settings. If the number of AF episodes is overestimated, than the number of successful device therapies for AF will also be overestimated. Other "AF derived" parameters, like atrial defibrillation threshold, will therefore in a number of cases refer to fast atrial tachycardias and atrial flutter.

In patients known with paroxysmal $\mathrm{AF}$ as the only documented arrhythmia before ICD implant, we observed the coexistence of more than one type of atrial arrhythmia. 
In this way device diagnostics can be helpful to identify patients being candidates for other types of hybrid therapy for AF.

The automatically allowed atrial therapies, antitachycardia pacing and the $50 \mathrm{~Hz}$ burst, applied early after onset of arrhythmias could terminate successfully $58 \%$ of atrial episodes. In addition $30 \%$ of arrhythmias terminated spontaneously. It is an interesting finding, that in patients with structural heart disease and documented AF, a high proportion of atrial arrhythmias can be terminated using painless therapies. It is suggestive, that the possibility of device therapy to be applied early after onset of arrhythmia was of importance for the therapy outcomes.

The contribution of AF among documented atrial arrhythmias was different in patients with different arrhythmia etiologies. It was $10 \%$ in patients with coronary artery disease and $80 \%$ in patients with dilated cardiomyopathy. The percentage of spontaneously terminating arrhythmias was much higher in patients with coronary artery disease than in patients with dilated cardiomyopathy. This suggests that a patient with a dilated cardiomyopathy has less chance to benefit from device therapy for atrial arrhythmias.

We found that the Jewel AF definitions used to classify atrial arrhythmias and therapy outcome need to be adjusted to bring them close to their clinical features and that device diagnostics need to be extended. These adjustments are required to improve the clinical and especially the investigational value of the Jewel AF.

In addition to different treatment modalities for atrial arrhythmias the Jewel AF has new pacing algorithms for mode switching and atrial arrhythmia prevention. Device therapy using extensive arrhythmia management requires consonant functioning of all algorithms to control arrhythmia. In case of pacing algorithms it is important that, in patients with atrial arrhythmias, these algorithms support maintenance of a stable sinus rhythm. The moment following successful therapy of atrial arrhythmias deserves special attention as far as mode of pacing is concerned. After termination of AF the electrophysiologic properties of the atria make them vulnerable to premature beats causing early reinitiation of arrhythmia. In chapter 6 the first study is presented to evaluate the influence of the mode switching rate stabilization pacing algorithms, mode switching fall and mode switching dwell, on atrial rate stability following termination of atrial arrhythmias in patients with Jewel AF.

We found that the dual chamber mode switching rate stabilization pacing following the arrhythmia termination resulted in dynamic DDI pacing in the presence of sinus rhythm and atrial premature beats in $23 \%$ of episodes and in fallback pacing in $18 \%$ of episodes. The algorithm can only under certain circumstances function as atrial rate smoothing. This means that although the basic pacing mode is DDD and the atrial arrhythmia prevention algorithm (atrial rate stabilization, the ARS) is used, pacing immediately after atrial arrhythmia termination is provided in a ventricular- timing- 
based mode. Moreover, the duration of mode switching rate stabilization pacing was often prolonged, because of device-based definition of atrial arrhythmia termination. This, in addition to the long en fixed duration of the postventricular atrial refractory period, limited the time during which the true atrial arrhythmia prevention pacing could operate. Depending on the time relation of the underlying rhythm and the pacing parameters (some of them are fixed when mode switching is used), the mode switching rate stabilization could even be the cause of iatrogenic atrial rate irregularity. Stabilizing atrial rhythm at the desired time and manner would require adjustment of the functioning and availability of atrial rate stabilization algorithms applied in present devices. At the moment we suggest using complex pacing algorithms only, when clinical advantages are expected and can be proven while these algorithms are programmed. Furthermore, if the device diagnostics do not allow insight in the functioning of the algorithms, it is useful to supplement the diagnostics by the Holter registrations.

These observations have also implications for the design of studies on pacing for arrhythmia prevention. So far, the studies on the effect of overdrive atrial pacing on recurrences of atrial arrhythmia do not lead to unanimous conclusions (26-32). We suggested some reasons, why this could be the case. Except from the obvious question: has a pacing algorithm effect on the atrial arrhythmia, other questions are also important. Has an algorithm the chance to operate in a patient, given his underlying rhythm. Do the programming settings limit the application of the algorithm in practice. Is an algorithm available in circumstances that are important for maintenance of sinus rhythm, such as during APBs or immediately after arrhythmia termination. Can proarrhythmia be expected due to the timing of algorithm related pacing. Can, after initially promising results, proarrhythmia occur at long term, for example due to permanently higher pacing rates. If an algorithm does not result in the expected benefits, is it due to failure of the concept or failure of the environment in which it operates. We made several suggestions on features desirable for the functioning and accessibility of the pacing algorithms that stabilize atrial rhythm.

Documenting interactions between the spontaneous rhythm and the pacing algorithms would give better insight in the functioning of the algorithms and allow better selection of patients who might benefit from pacing for arrhythmia prevention. Devices, in which these algorithms are being applied, should provide adequate diagnostics to evaluate algorithm operation and not only arrhythmia counters.

These considerations about the true value of algorithm and proper patient selection are especially important in patients in whom not the ventricular arrhythmia or bradycardia is present, but where arrhythmia prevention is the only indication for implant. 
Till now, better outcomes were reported in studies with subset of patients in whom the mechanism of atrial fibrillation was elucidated before applying atrial pacing $(33,34)$.

Atrial arrhythmia prevention in the Jewel AF uses two new pacing algorithms. The Atrial Rate Stabilization, operating outside arrhythmic episode, is designed to prevent the onset of atrial tachyarrhythmias caused by atrial premature beats, followed by pauses and short-long sequences at the atrial level. The Overdrive Switchback Delay is designed to prevent atrial arrhythmia reinitiation caused by atrial premature beats immediately after arrhythmia termination. These two preventive therapies together with the termination therapies (antitachycardia pacing, the $50 \mathrm{~Hz}$ burst, cardioversion) form the device-based atrial arrhythmia therapy. That extensive atrial arrhythmia therapy was assessed (chapter 7) for its ability to reduce the burden of spontaneous atrial arrhythmias, AF/AT, in 52 patients with the Jewel AF. The atrial therapies resulted in a significant reduction of time that the ICD patient suffered from atrial arrhythmias and in a reduction of long lasting atrial episodes. Furthermore, there was a trend towards a reduction in the episode frequency. The findings suggest, that in patients with structural heart disease reducing atrial arrhythmia duration can only to some extend modify its occurrence. The arrhythmia burden reduction was highly variable among patients and tended to be greatest in patients with the highest arrhythmia burden. This stresses the importance of arrhythmia history documentation as part of the preimplant patient evaluation, before device choice is made. Importantly, a significant arrhythmia burden reduction persisted in patients in whom only pacing therapies (so no cardioversion shocks) were delivered. This means that in ICD patients, a device with pacing and shock therapies has more clinical utility compared to a shock-only device. Furthermore, programming only painless pacing therapies to be delivered automatically, while cardioversions are performed under short-lasting anesthesia in hospital, do not limit the value of device therapy for atrial arrhythmias.

Dual chamber ICD technology was a very welcome development for patients with ventricular arrhythmia who, due to their underlying heart disease or use of antiarrhythmics, suffered from AV conduction disturbances, sinus bradycardia and chronotropic incompetence. Previously, in pacemaker-dependent recipients of single chamber ICDs who were dependent on (more sophisticated) cardiac stimulation, separate pacing systems were implanted in some centers. That solution could lead to device-device interaction with potentially catastrophic sequelae (35-39). Thanks to the currently available technology of pacing integrated in the ICD system those life-threatening interactions can now be avoided. However, also in modern devices the ICD and the pacemaker cannot function independently from each other due to safety of both counterparts of the system. The requirement that detection of ventric- 
ular arrhythmias should never be jeopardized by heart stimulation, has consequences for the availability of pacing rates, and duration of blanking and refractory periods in pacemaker. In addition, modern dual chamber arrhythmia management devices have new pacing algorithms for atrial and ventricular arrhythmia prevention and rate stabilization that were not available in previous pacemakers. In this thesis (chapter 8) several examples of device-device interactions in four models of dual chamber ICDs are discussed. Illustrated are cases when the interactions between various pacemaker features and ICD caused respectively: inappropriate arrhythmia detection, inappropriate pacing, apparent pacemaker tachycardia and arrhythmia induction. The interactions are not of mechanical or technical, but of logical nature. They occur during arrhythmia detection or outside an arrhythmia episode when the heart rate is higher. Under these circumstances the detection windows become critically dependent on the duration of blanking and refractory periods of both the ICD and the pacemaker. We found that the potentially proarrhythmic interactions are not always prevented by parameter interlocks. Furthermore, the interactions are inapparent during normal sinus rhythm and cannot be predicted then. This means that exercise testing and arrhythmia induction study after ICD implantation are valuable to evaluate the interactions and allows taking programming measures to limit the chance of interactions. Especially complex pacing algorithms should be programmed with caution and consideration of their true clinical value. Sensing and pacing related phenomena have very different consequences in both the ICD and the pacemaker. Therefore observations from bradycardia devices cannot simply be extrapolated to the ICD integrated pacemaker.

\section{FINAL REMARKS}

The diagnostic and therapeutic advantages of current dual chamber ICDs were achieved at the cost of increasing device complexity. In spite of the possibility of many ICD functions operating automatically, control and programming by the physician is necessary to prevent problematic settings, while using the best of the advanced ICD technology. In this way the new possibilities of arrhythmia therapy offered by the dual chamber ICD are used with optimal benefit for the patient. Observations on dual chamber ICD algorithms for arrhythmia detection, therapy and prevention contribute to advancements of arrhythmia management using device therapy. 


\section{REFERENCES}

1. Myerburg R, Kessker K, Castellanos A. Sudden Cardiac Death: structure, function and time dependence of risk. Circulation 1992 (Suppl); 85:1-2.

2. Bayes de Luna A, Coumel P, Leclercq J. Ambulatory sudden cardiac death: mechanism of production of fatal arrhythmia on the basis of data from 157 cases. Am Heart J 117; 1989:151-159.

3. The Antiarrhythmics versus Implantable Defibrillators (AVID) Investigators. A comparison of anti-arrhythmic drug therapy with implantable defibrillators in patients resuscitated from near-fatal ventricular arrhythmias. N Engl J Med 1997; 337: 1576-83.

4. Anderson J, Hallstorm A, Epstein A, et al. The AVID Investigators. Design and results of the Antiarrhythmics vs. Implantable Defibrillator (AVID) Registry. Circulation 1999; 99: 1692-1699.

5. Moss A, Hall W, Cannom D, et al, for the Multicenter Automatic Defibrillator Implantation Trial Investigators. Improved Survival with an Implantable Cardioverter Defibrillator in Patients with Coronary Disease at High R isk for Ventricular Arrhythmias. N Engl J Med 1996; 335: 1993-1940.

6. Kuck K, Cappato R, Siebels J, Ruppel R. Randomized Comparison of Antiarrhythmic Drug Therapy With Implantable Defibrillator in Patients Resuscitated from Cardiac Arrest: the Cardiac Arrest Study of Hamburg (CASH). Circulation 2000; 102:748-54.

7. Connoly S, Gent M, Roberts R, et al. on behalf of the CIDS Investigators. Canadian Implantable Defibrillator Study (CIDS): A Randomized Trial of the Implantable Cardioverter Defibrillator against Amiodarone. Circulation 2000; 101: 1297.

8. Swerdlow $C$, Chen $P$, Kass R, et al. Discrimination of ventricular tachycardia from sinus tachycardia and atrial fibrillation in tired-therapy cardioverter-defibrillator. J Am Coll Card 1994; 23: $1342-1355$.

9. Swerdlow C, Ahern T, Chen P, et al. Underdetection of ventricular tachyarrhythmia by algorithms to enhance specificity in a tired-therapy cardioverter-defibrillator.J Am Coll Card 1994; 24: 416-424.

10. Neutzner J, Pitschner H, Schlepper M. Programmable VT detection enhancements in implantable defibrillator therapy. PACE 1995; 18: 539-547.

11. Brachmann J. Swerdlow C, Mitchell B, et al. for the Worldwide 7218 ICD investigators. Worldwide experience with the electrogram width feature for improved detection in an implantable pacer-cardioverter- defibrillator. J Am Coll Card 1997; 28:115A.

12. Brugada J, Mont L, Figueiredo $M$, et al. Enhanced detection criteria in implantable defibrillators. J Cardiovasc Electrophysiol 1998; 9: 261-268.

13. Birgersdotter-Green $U$, Rosenquist $M$, Lindemans $F$, et al. Holter documented sudden death in a patient with an implanted defibrillator. PACE 1992: 15: 1008-1014.

14. Fan K, Lee K, Lau C.P. Dual chamber implantable cardioverter defibrillator: benefits and limitations. J Interv Card Electrophysiol 1999; 3:239-45

15. Wellens $\mathrm{H}$, Bar $\mathbf{F}$, Farre $\mathbf{J}$, et al. Initiation and termination of ventricular tachycardia by supraventricular stimuli. Am J Cardiol. 1980; 46:576-82.

16. Belhassen B, Shapira I, Kauli N, et al. Initiation and termination of ventricular tachycardia by supraventricular beats. Basic Res Cardiol 1980:75:772-9. 
17. Berman ND. Ventricular tachycardia initiated by both normally and aberrantly conducted atria premature beats. PACE 1980; 3:519-25.

18. Bekheit S, Turitto $G$, Fontaine J, el-Sherif $N$. Initiation of ventricular fibrillation by supraventricular beats in patients with acute myocardial infarction. Br Heart J. 1988; 59:190-5.

19. Brembilla-Perrot B, Gretzinger A, Guenoun $P$, et al. Initiation of ventricular fibrillation by atrial fibrillation. Eur Heart J. 1994; 15:289-91.

20. Pruvot $E$, Thonet $G$, Vesin J, et al. Heart rate dynamics at the onset of ventricular tachycardias as retrieved from implantable cardioverter-defibrillators in patients with coronary artery disease. Circulation 2000; 101:2398-2404.

21. Smith M, Ellenbogen K, Beightol L, Eckberg D. Sympathetic neural responses to induced ventricular tachycardia. J Am Coll Cardiol 1991; 18:1015-24.

22. Smith M, Joglar J, Wasmund S, et al. Reflex control of sympathetic activity during simulated ventricular tachycardia in humans. Circulation. 1999; 100:628- 634.

23. Best $P$, Hayes $D$, Stanton $M$. The potential usage of dual chamber pacing in patients with implantable cardioverter defibrillators. PACE 1999; 22:79-85.

24. Dries D, Exner D, Gersh B, et al. Atrial fibrillation is associated with an increased risk for mortality and heart failure progression in patients with asymptomatic and symptomatic left ventricular systolic dysfunction: a retrospective analysis of the SOLVD trials. Studies of Left Ventricular Dysfunction. J Am Coll Cardiol 1998; 32:695-703.

25. Gillis A, Wyse G, Connolly $S$, et al. Atrial pacing periablation for prevention of paroxysmal atrial fibrillation. Circulation 1999; 99:2553-2558.

26. Hill M, Dinsmmoor D, Klecker K, Gillis A. Impact of atrial pacing on atrial fibrillation onsets in the PA3 trial. Circulation 1998; 17 (suppl I):711(A).

27. Ricci R, Puglisi A, Azzolini P, et al. Consistent atrial pacing algorithm to suppress recurrent paroxysmal atrial fibrillation: a randomized prospective cross over study. PACE 21 (II): 798, 1998.

28. Greenberg MD, Katz NM, Iuliano S, et al. Atrial pacing for the prevention of atrial fibrillation after cardiovascular surgery. J Am Coll Cardiol. 2000 May; 35(6): 1416-22.

29. Blommaert D, Gonzalez M, Mucumbitsi J, et al. Effective prevention of atrial fibrillation by continuous atrial overdrive pacing after coronary artery bypass surgery. J Am Coll Cardiol. 2000 May; 35(6): 1411-5.

30. Chung $M$, Augostini R, Asher $C$, et al. Ineffectivness and potential proarrhythmia of atrial pacing for atrial fibrillation after coronary artery bypass grafting. Ann Thorac Surg. 2000 Apr; 69(4): 1057-63.

31. Kurz D, Naegeli B, Kuntz M, et al. Epicardial biatrial synchronous pacing for prevention of atrial fibrillation after cardiac surgery. PACE 1999; 22:721-726.

32. Anselme F, Saoudi N, Cribier A. Pacing in prevention of atrial fibrillation: The PIPAF studies.J Interv Card Electrophysiol. 2000 Jan; 4 Suppl 1:177 184.

33. Coumel P, Friocourt P, Mugica J, et al. Long-term prevention of vagal atrial arrhythmias by atrial pacing at 90/minute: experience with 6 cases. PACE 1983; 6:552-560.

34. Attuel P, Pellerin D, Mugica J, Coumel P. DDD pacing: an effective treatment modality for recurrent atrial arrhythmias. PACE 1988; 11:1647-1654.

35. Fogoros RN: Device-device interactions. In: Naccarelli $G$ and Veltri E, Eds: Implantable Cardioverter-Defibrillators, Blackwell Scientific Publications, Boston 1993, pp205-215. 
36. Singer I, Slater D: Interactions of implantable cardioverter-defibrillators with antiarrhythmic drugs and pacemakers. In: Singer I, Ed: Implantable Cardioverter Defibrillator. Futura Publishing Company, Armonk 1994, pp377-388.

37. Sheahan RG, Dorian P, Poludnikiewicz M, Newman D. Dual device therapy in the setting of changing ICD technology: device-device interaction revisited. PACE 1997; 20:1704-7.

38. Brode SE, Schwartzman D, Callans DJ, et al. ICD-antiarrhythmic drug and ICD-pacemaker interactions. J Cardiovasc Electrophysiol. 1997; 8: 830-42.

39. Geiger MJ, O'Neill P, Sharma A, et al. Interactions between transvenous nonthoracotomy cardioverter defibrillator systems and permanent transvenous endocardial pacemakers. PACE 1997; 20: 624-30. 


\section{Summary}

In this thesis studies are presented on the technical functioning and clinical results of new algorithms used in the last generation of dual chamber ICDs, to manage cardiac arrhythmias.

Chapter 1 discussed the development and current status of ICD technology. It covered basic principles of arrhythmia treatment using ICD therapy. Important ICD functions, such as arrhythmia detection, the different electrical therapies, device testing, and arrhythmia analysis using diagnostics and memory of the device are illustrated by ICD rhythm registrations. These ICD functions are described as they have developed over the years, from the earliest defibrillators to the most advanced dual chamber arrhythmia management devices.

Chapter 2 gave information on the ICD patients treated in the Academic Hospital Maastricht. This includes their demographics, clinical characteristics, and arrhythmia history before ICD implantation. It also discusses the use of the device after implant. Importantly, it shows that continuously occurring refinements in ICD therapy led to the expanding use of the device in our hospital during the past 15 years.

In chapter 3 the new dual chamber arrhythmia detection algorithm, the PR Logic, was evaluated in a subset of diagnostically difficult arrhythmias. In these arrhythmias, such as double tachycardias (SVT+VT), or SVT's occurring in the VT/VF zone, the ventricular rate only is an insufficient criterion for classification. The detection of ventricular arrhythmias remained secured when applying the PR Logic algorithm. The arrhythmia discrimination, although improved when compared to single chamber devices, is still not perfect. Causes of diagnostic problems are discussed and divided into three groups: algorithm failure, algorithm limitation, and programming settings. Furthermore, programming measures to avoid inappropriate detection in present ICDs are discussed. Using observations from this study and from the literature, suggestions are made to improve detection specificity in future devices.

Chapter 4 looked at the dual chamber ICD from a different perspective than done so far. The diagnostic possibilities of the dual chamber ICD allow better insight in the mechanisms contributing to the onset and maintenance of ventricular arrhythmias. Information from the atrial channel is useful to guide antiarrhythmic drug therapy of ventricular arrhythmias by influencing their occurrence and susceptibility to electrical 
therapies. Furthermore, dual chamber diagnostics allow better programming of the ICD detection zones. In this way the advantages of dual chamber ICD go beyond avoidance of shortcomings of the single chamber system.

Detection and therapy of atrial arrhythmias is a new important function of the dual chamber ICDs and became first available in the 7250 Jewel AF (Medtronic). In chapter 5 atrial arrhythmias and device interventions were studied in patients with diseased hearts, a history of VT/VF, on class III antiarrhythmics, and implanted with the 7250 Jewel AF. The atrial arrhythmias documented early after their onset had, in most cases, a longer atrial cycle length than during atrial fibrillation, also in patients in whom atrial fibrillation was the only atrial arrhythmia clinically documented before ICD implant. Half of these arrhythmias could be teminated with pacing therapies, one third terminated spontaneously. No ventricular proarrhythmia was observed while applying atrial therapies. Dual chamber devices capable of monitoring and treating atrial arrhythmias provide information about the natural history of the arrhythmia and are potentially able to influence the natural course of atrial arrhythmias by electrical therapy early after their onset.

Feasibility and safety of an extensive atrial arrhythmia management program in the dual chamber ICDs require correct and synergic functioning of different algorithms to control arrhythmias. In chapter $\mathbf{6}$ functioning of new mode switching rate stabilization pacing algorithms was evaluated. Rate stabilization pacing provided in the modified DDI mode is not based on the timing of atrial events. The algorithm can only under certain circumstances function as atrial rate stabilization pacing as well. Both the potentially beneficial and proarrhythmic effects of this algorithm are discussed and illustrated. Furthermore, the duration of time after atrial arrhythmia termination, during which the mode switching rate stabilization algorithm is applied, is often prolonged due to the device-based definition of arrhythmia termination. This limits the time during which true Atrial Rate Stabilization pacing can operate. The clinical performance of pacing as part of device therapy for atrial arrhythmias could improve from adjustments in availability and functioning of rate stabilization algorithms.

The Jewel AF is the first implantable device in which both termination therapies for atrial arrhythmias and pacing algorithms for atrial arrhythmia prevention are available. In chapter 7 the effects of such a combined atrial arrhythmia treatment on the arrhythmia burden (the time during which the patient suffers from the atrial arrhythmia), were studied in patients with paroxysmal atrial arrhythmias and an ICD indication. Termination therapies used one or more of three modalities: adaptive antitachycardia pacing, $50 \mathrm{~Hz}$ burst or cardioversion. The preventive therapy is a dual chamber pacing algorithm, the Atrial Rate Stabilization. The results of the first 
multicenter (including ours) Jewel AF study are presented. The atrial arrhythmia burden is reduced as shown by shorter, and possibly fewer, episodes with this therapy.

Extended arrhythmia detection capabilities in dual chamber ICDs together with advanced pacing algorithms can give rise to new types of device-device interactions. Some of the possible interactions occurring in different dual chamber ICD models were illustrated in chapter 8 . The requirement that detection of ventricular arrhythmias should never be jeopardized by cardiac stimulation, has and should always influence functioning of an ICD integrated pacemaker. The interactions become more complex due to the fact that modern dual chamber arrhythmia management devices have pacing algorithms for atrial and ventricular arrhythmia prevention and for rate stabilization that were previously not available in pacemakers. Careful evaluation of these new features is required in the individual patient. 
[212] 


\section{Samenvatting}

Het concept van de implantable cardioverter defibrillator (ICD) heeft een dynamische ontwikkeling doorgemaakt. De eerste generatie implantable defibrillatoren waren in staat om levensbedreigende kamerritmestoornissen te herkennen en tijdig te onderbreken. In latere generaties ICDs werden echter functies toegevoegd, die gericht waren op de behandeling van ritmestoornissen (aritmieen) in bredere zin. In dit proefschrift worden bevindingen beschreven bij patiënten bij wie in het Academisch Ziekenhuis Maastricht een ICD werd geïmplanteerd. In het bijzonder, de nieuwe mogelijkheden die de twee kamer ICD technologie biedt voor het behandelen van de hartritmestoornissen op supraventriculair and ventriculair niveau. In dat kader worden studies beschreven over het technisch functioneren en de klinische resultaten van de nieuwe algoritmes voor detectie, therapie en preventie van ventriculaire en supraventriculaire aritmieen. Tevens worden er suggesties gedaan voor aanpassingen en veranderingen van de tweekamer algoritmes om nieuwe mogelijkheden van ICD therapie verder te ontwikkelen.

Hoofdstuk 1 geeft een korte inleiding over de geschiedenis en de werkingswijze van de ICD en introduceert de ICD vocabulaire, die in latere hoofdstukken wordt gebruikt. De verschillende ICD functies worden benaderd vanuit de optiek van de ICD ontwikkeling tot steeds complexer wordende apparaten. Behandeld worden de principes van detectie en het onderscheid maken tussen verschillende vormen van aritmieen, de therapieën voor kamer- en boezem ritmestoornissen, de pacemaker functie, het testen van het apparaat en de mogelijkheden van het "geheugen" van de ICD. De evolutie van deze functies vanaf het eerste eenvoudige eenkamer systeem, tot de nieuwste tweekamer apparaten, wordt geillustreerd aan de hand van ICD ritme registraties.

Hoofdstuk 2 beschrijft kort de ICD patiënten populatie in het AZM. Het geeft een overzicht van de klinische karakteristieken en aritmie geschiedenis van deze patiënten voorafgaand aan de ICD implantatie. De operatieve technieken voor verschillende ICD systemen worden genoemd. De follow-up van de aritmieen en de hiervoor toegepaste ICD therapieën na de implantatie worden beschreven. De veranderingen in de ICD therapie door de jaren heen, die het gevolg zijn van technische vooruitgang en uitbreiding van indicaties voor de ICD therapie worden geschetst. 
In hoofdstuk 3, wordt het nieuwe tweekamer detectie algoritme, de PR Logic, geëvalueerd in een groep patiënten met moeilijk te diagnosticeren aritmieen. Bij deze aritmieen waren gelijktijdig ventriculaire en supraventriculaire ritmestoornissen (VT + SVT) aanwezig, of een SVT in de VT zone. Hierbij is de snelheid van het ventriculaire ritme zelf onvoldoende voor juiste classificatie. Met het PR Logic algoritme blijft de detectie van de ventriculaire ritmestoornissen betrouwbaar, ook wanneer er tegelijkertijd een SVT aanwezig is. De discriminatie tussen VT en SVT, hoewel verbeterd t.o.v. eenkamer systemen, werkt nog niet onder alle omstandigheden. Oorzaken van diagnostische problemen worden geanalyseerd en aanpassingen in de programmering binnen het bestaande algoritme worden besproken. Op grond van deze studie en de literatuur worden suggesties gedaan om de detectie specificiteit te verbeteren in toekomstige algoritmes.

In hoofdstuk 4 wordt de tweekamer ICD technologie vanuit een andere perspectief beschouwd dan gebruikelijk in de literatuur (het onderscheid tussen VT en SVT en de mogelijkheden van DDD pacing). Gekeken wordt naar de toegevoegde waarde van de diagnostische mogelijkheden van de tweekamer ICD voor de behandeling van kamerritmestoornissen. De extra waarde ligt in een beter inzicht in het mechanisme van het ontstaan en het zich handhaven van de ventriculaire aritmieen. Hierdoor kunnen deze ritmestoornissen behandeld worden met medicamenten in combinatie met de ICD. Zowel de recidieven van ventriculaire aritmieen als hun respons op device therapieen worden in gunstige zin beïnvloed. Voorts kunnen de aritmie detectie criteria in de ICD beter worden ingesteld.

In hoofdstuk 5 wordt een geheel nieuw toepassing van de ICD therapie beschreven: de detectie en behandeling van atriale ritmestoornissen. Dit gebeurt aan de hand van ervaringen met de Jewel AF - de eerste ICD die mogelijkheden heeft voor ritme detectie en therapie op tweekamer niveau. Deze twee kamer ICD therapie werd toegepast bij patiënten met levensbedreigende kamerritmestoornissen, en paroxysmaal atrium fibrilleren bij verschillende vormen van onderliggend hartlijden die behandeld werden met klasse III antiarrhythmica. De registratie van recidiverende atriale aritmieen, die werden gemaakt kort na hun ontstaan, lieten in de meeste gevallen een langere atriale cyclus lengte zien dan tijdens atrium fibrilleren. Indien deze aritmieen kort na hun ontstaan ook werden behandeld, konden zij in de helft van de gevallen getermineerd worden met verschillende antitachycardia pacing therapieën in het atrium. Een derde van de aritmie episoden stopten spontaan. Er werden geen, door de atriale therapie geïnduceerde, ventriculaire ritmestoornissen waargenomen. De sterke en zwakke punten van de atrial aritmie detectie van de ICD worden besproken. 
Hoofdstuk 6 gaat in op een ander aspect van de anpak van atriale ritmestoornissen door dezelfde, tweekamer Jewel AF ICD. Het behandelt de waarde van de nieuwe mode switching rate stabilization pacing algorithme voor stabiliteit van het boezem ritme. Deze vorm van pacing kan alleen onder bepaalde omstandigheden fungeren als een algoritme dat onregelmatigheden van het boezemritme kan opvangen. Voorts wordt het algoritme vaak langere tijd toegepast, dan alleen direct na het beëindigen van atriale aritmie. Suggesties worden gedaan voor veranderingen van de rate stabilization algoritmes om synergisme met de pacing algoritmes voor preventie van atriale aritmieen te verbeteren.

Behandeling van atriale ritme stoornissen, waarbij terminatie en preventie therapieen met elkaar gecombineerd kunnen worden, kwam voor het eerst beschikbaar in de Jewel AF. De effecten van deze twee modaliteiten op de duur en frequentie van atriale aritmieen (de zogenaamde "atrial arrhythmia burden") zijn het onderwerp van hoofdstuk 7. Beschreven zijn de resultaten van de eerste multicenter (waaronder ons centrum) Jewel AF studie bij patiënten met een ICD indicatie en paroxymale atriale ritmestoornissen. De behandeling van de atriale ritmestoornissen met pacing and preventie therapieen tezamen, leidt tot een reductie in duur en mogelijk ook in aantal recidieven van de atriale aritmieen.

Hoofdstuk 8 gaat over het functioneren van de tweekamer pacemaker geïntegreerd in een tweekamer ICD. Er worden nieuwe vormen van interactie tussen de tachy- en bradycardia onderdelen van de ICD beschreven. Deze interacties zijn deels toe te schrijven aan de basale ICD filosofie om de detectie van kamerritmestoornissen onder alle omstandigheden te beschermen. In de nieuwe ICDs worden de interacties complexer door de aanwezigheid van twee detectie niveaus, en door de moderne pacing algoritmes ter preventie van atriale en ventriculaire aritmieen.

\section{SLOTOPMERKING}

De twee kamer ICD technologie biedt vele nieuwe en veelzijdige mogelijkheden voor het behandelen van de hartritmestoornissen. Dit heeft geleid tot toenemende complexiteit van deze apparaten. Ondanks de vele automatische functies in een ICD, blijft aandacht voor het programmeren nodig, om optimaal gebruik te maken van deze nieuwe mogelijkheden. Belangrijk is niet alleen het volgen, maar ook iedere keer het vertalen van de technische ontwikkelingen naar hun klinische betekenis om op die manier tot optimale behandeling van de patiënt te komen. 


\section{Dankwoord}

De toekomst en het heden zijn geworteld in het verleden. Vandaar dat dit proefschrift voor iedereen zijn bijzondere betekenis heeft.

Lieve Hans, het obligate dankwoord aan de partner na afronding van een proefschrift zou tekort doen aan de onvoorwaardelijke steun die ik van jou heb ervaren bij alles wat daaraan vooraf ging. De opleiding tot cardioloog in Maastricht, tijden van extreme drukte in mijn werk, twee kinderen, tezamen met je eigen professionele carrière (in Eindhoven nota bene), vragen om exceptioneel begrip en verdraagzaamheid. Ik heb dit alles kunnen bereiken, omdat ik zo veel van jou gekregen heb.

Professor Wellens, eminent wetenschapper en leermeester, wil ik bedanken voor alle kansen die hij mij heeft geboden. Een excellente opleiding tot cardioloog met daarna de mogelijkheid om een fascinerende discipline binnen de cardiologie te beoefenen. Zijn kennis van de "state of the art" cardiologie, veelzijdige interesse in alle facetten van ons vakgebied, zijn visie op alles wat nieuw en de moeite waard is, onverslijtbaar enthousiasme, zijn voor mij inspirerend geweest. Als promotor van het proefschrift, waren zijn vriendelijke beschikbaarheid, tijd, constructieve kritiek, geduld, hoge werk tempo, telkens stimulerend om door te gaan.

Gaarne wil ik de leden van de beoordelingscommissie, Dr. H.J.G.M. Crijns (voorzitter), Dr. A.P.M Gorgels, Prof. Dr. R.N.W. Hauer, Prof. Dr. L. Jordaens, Prof. Dr. B. Mochtar, bedanken voor de tijd en aandacht die ze hebben besteed aan het beoordelen van het manuscript.

Mijn maatjes van de ritmecluster ben ik dank verschuldigd voor het overnemen van mijn klinische taken gedurende de drie maanden waarin ik de belangrijkste hoofdstukken van mijn proefschrift heb kunnen schrijven. Charles Kirchhof stond klaar, niet alleen als het alledaagse werk gedaan moest worden, maar iedere keer wanneer de belangen van onze pacemaker/ICD club verdedigd moesten worden. Joep Smeets heeft op een voor mij belangrijk moment, het ritme cluster dusdanig organisatorisch vorm gegeven dat er ruimte ontstond voor mijn promoveren. Luz-Maria Rodriguez heeft me de weg gewezen in het "promoveerland", door mij van vele praktische adviezen te voorzien. 
Karel Den Dulk was degene die ervoor zorgde dat hartstimulatie als onderdeel van de cardiologie op hoog niveau werd bedreven. Het was bijzonder om op zo'n afdeling te mogen beginnen.

De cardiochirurgen hebben de ontwikkeling van de ICD therapie vanaf het begin van dichtbij meegemaakt. De eerste generatie defibrillatoren werden door hen via thoracotomie geplaatst. Later, toen de transveneuze systemen door de cardioloog geïmplanteerd konden worden, waren ze altijd bereid om ons te helpen bij revisies van de oudere systemen. Ze staan ons bij tijdens risicovolle procedures die ook altijd goed zijn verlopen. Hetzelfde krediet verdienen ook de anesthesiologen. Ondanks, dat we in de meeste gevallen patiënten moeten aanbieden, die een hoger risico hebben, is de narcose bij iedere patient zonder uitzondering goed en ongecompliceerd verlopen. Dankzij het hele team mensen op de OK is Maastricht nog steeds (een van de weinige) ICD centrum met een peri- en postoperatieve mortaliteit van $0 \%$.

De meesten van mijn collegae met wie ik nu samenwerk hebben in vroegere jaren, door kennisoverdracht in verschillende disciplines van de cardiologie, bijgedragen aan mijn vorming als cardioloog. Miel, bedankt voor je betrokkenheid en wijze raadgeving in het verleden en nu. Simon, bedankt voor alle computer software voor grafische en statistische programma's; het heeft me veel zelfstandigheid gegeven bij het promoveren.

Daar het proefschrift gebaseerd is op klinisch wetenschappelijk onderzoek, hebben klinische werkzaamheden een belangrijke rol gespeeld. Vanaf de eerste ICD patiënt in Maastricht tot op heden zijn er mensen geweest die heel belangrijk waren voor de ICD therapie in ons centrum.

De technici van de Hartfunctieafdeling: Irma, Luuk, Michel, Thieu, Sylvia, Ron, voelen zich enorm verantwoordelijk voor de ICD patiënten. De technici hebben alle kwaliteiten die zo onontbeerlijk zijn voor het behandelen van patiënten die afhankelijk zijn van de moderne technologie. Ze zijn bekwaam en menselijk, ze hebben het druk en zijn toch flexibel en altijd gemotiveerd om tijd te investeren in verbeteringen. Dat niet een ICD bij geen enkele patiënt in ons centrum het ooit heeft laten afweten is met name aan hen te danken.

De verpleegkundigen op de afdeling van Jean Partouns besteden veel aandacht aan de patiënten met ernstige kamerritmestoornissen die geëvalueerd worden voor de ICD implantatie. Hun humane en vriendelijke benadering van de patiënten, speelt een belangrijke rol in de acceptatie van deze therapie. Deze aanpak wordt door de vele ICD patiënten nog lange tijd na de implantatie als uniek beoordeeld. Marianne heeft veel tijd besteed aan de psychosociale aspecten van de ICD therapie. 
De laatste tijd worden steeds meer ICD patiënten op de afdeling B3 voorbereid voor de ingreep, waar ze goed verzorgd en begeleid worden.

De assistenten die op de afdeling of tijdens hun diensten (vaak in de nachtelijke uren) de ICD patiënten behandeld hebben, wil ik eveneens bedanken voor hun inspanning voor deze, in vele opzichten bewerkelijke, groep patiënten.

De secretaressen spelen een belangrijke rol bij de klinische en poliklinische ICD patiënten administratie. Nicole heeft de gegevens rondom de ICD studies professioneel bijgehouden.

Voor de ICD patiënten heb ik veel respect voor de dappere wijze, waarop ze met hun doorgemaakte en vaak nog terugkerende ritmestoornissen omgaan. Respect, dat ze ondanks restricties er alles aan doen een waardevol leven te leiden en voor anderen van betekenis zijn. De belangstelling die ik van velen heb mogen ervaren met betrekking tot de vorderingen rondom mijn promotie waren hartverwarmend.

Mijn bekenden van Guidant, Medtronic en Vitatron, wil ik bedanken voor de samenwerking, ondersteuning van educatie en studies binnen onze afdeling en hun sponsoring van dit proefschrift.

Van mijn ouders en mijn zus heb ik zonder enig twijfel heel veel gekregen, niet alleen vroeger, maar ook later in mijn volwassen leven. Ze hebben altijd vertrouwen gehad in de keuzes die ik in mijn leven heb gemaakt, hoewel het niet altijd makkelijk voor hen is geweest. We hebben als gezin nooit tevergeefs een beroep op hen gedaan.

Robert en Mark, hopelijk zal ik jullie binnenkort kunnen geven, waar jullie niet alleen ten tijde van mijn promotie, maar ook in eerdere jaren recht op hadden en hebben. Robert, bedankt voor je hulp bij het voorbereiden van het manuscript.

Onze oppassen, die in de afgelopen jaren voor de kinderen (en hun ouders) gezorgd hebben, kunnen we niet voldoende bedanken. Met name Magda, die ten tijde van mijn promotie bij ons was, is buitengewoon lief voor ons gezin geweest. We wensen haar succes met haar eigen studie volgend jaar. 
[220] 


\title{
Curriculum vitae
}

\author{
Name $\quad$ Barbara Dijkman - Domanska. \\ Born 26 May 1960, Poznan, Poland. \\ 1978 Gymnasium B, Poznan. \\ 1978 - '84 MD Degree, Medical Faculty, University of Poznan. \\ 1985 - '86 Prespecialization trainingship, followed by registration as a \\ Medical Practitioner, University Hospital Poznan. \\ 1987 - '88 Dutch MD registration obtained after required one year clinical \\ work experience (Alkmaar Medical Center). \\ 1988 - '89 Resident Cardiology, Zuiderziekenhuis Rotterdam. \\ 1990 - '91 Resident Cardiology, Academic Hospital Maastricht, \\ during one year working in electrophysiology laboratory. \\ 1992 - '93 Resident Internal Medicine (St Maarten Gasthuis Venlo and \\ Maasland Ziekenhuis Sittard) as part of the cardiology \\ trainingship. \\ 1993 - '97 Specialization in Cardiology, Academic Hospital Maastricht; \\ Head: Prof. Dr. H.J.J.Wellens. \\ 1 April 1997 - Cardiologist in Academic Hospital Maastricht; \\ staff member, field of interest: ICDs and pacemakers.
}




\section{Publications}

\section{JOURNAL ARTICLES}

Dijkman B, Wellens H.J.J.

Importance of the Atrial Channel for Ventricular Arrhythmia Therapy in the Dual Chamber Implantable Cardioverter Defibrillator.

J Cardiovasc Electrophysiology 2000: 11: 1309-1319.

Dijkman B, Wellens H.J.J.

Diagnosis and Therapy of Atrial Tachyarrhythmias in the Dual Chamber Implantable Cardioverter Defibrillator.

J Cardiovasc Electrophysiology 2000: 11: 1196-1205.

Dijkman B, Wellens H.J.J.

Dual Chamber Arrhythmia Detection in the Implantable Cardioverter Defibrillator J Cardiovasc Electrophysiology 2000; 11:1105-1115.

Dijkman B, Wellens H.J.J.

Merits and Limitations of the Mode Switching Rate Stabilization Algorithms in the Dual Chamber Implantable Cardioverter Defibrillator.

J Interv Cardiac Electrophysiology 2001, in press.

Dijkman B, Wellens H.J.J.

Interactions Between Pacing and Arrhythmia Detection Algorithms in the Dual Chamber Implantable Cardioverter Defibrillator

J Interv Cardiac Electrophysiology 2001, in press.

Friedman P, Dijkman B, Warman E, Xia HA, Mehra R, Staton M, Hammill S, For the Worldwide Jewel AF Investigators.

Atrial Therapies Reduce Atrial Arrhythmia Burden in Defibrillator Patients.

Circulation 2001, submitted.

Swerdlow C, Schols W, Dijkman B, Jung W, Sheth N, Olson W, Gunderson B. Detection of Atrial Fibrillation and Flutter by a Dual Chamber Implantable Cardioverter Defibrillator. For the Worldwide Jewel AF Investigators.

Circulation 2000; 101:878-85. 
Timmermans C, Rodriquez LM, Den Dulk K, Dijkman B, SmeetsJ, Wellens HJJ. Cure of Incessant Pacemaker Circus Movement Tachycardia by Radiofrequency Catheter Ablation.

J Cardiovasc Electrophysiology 1996; 7: 862-6.

Dijkman B, Den Dulk K, Wellens H.J.J.

Management of Electrical Instability After ICD Implantation.

PACE 1995:18:148-152.

Den Dulk K, Dijkman B, Pieterse M, Wellens H.J.J.

Initial Experience with Mode Switching in a Dual Sensor, Dual Chamber Pacemaker in Patients with Paroxysmal Atrial Tachyarrhythmias.

PACE 1994; 17:1900-1907.

Gorgels A, Vos M, Leunissen J, Dijkman B, Wellens H.J.J.

Flunarizine as a Specific Drug to Identify Triggered Activity Based on Delayed Afterdepolarization.

In: Tachycardias: Mechanisms and Management, p 87-97. Ed: M.E. Josephson, H.J.J Wellens. Futura Publishing Company 1993.

Rodriquez L, Smeets J, Schlaepfer J, Katsivas A, Dijkman B, De Chillou Ch, Wellens H.J.J. Radiofrequency Catheter Ablation of Three Accessory Pathways in a Single Session.

J Cardiovasc Electrophysiology 1992; 3: 141-149.

\section{ABSTRACTS}

Dijkman B, Wellens H.J.J.

Pacing Termination of Atrial Recurrences in ICD Patients With a History of Paroxysmal Atrial Fibrillation.

PACE 2000; 23: 416 (A).

Dijkman B, Wellens H.J.J.

Importance of the Far Field Sensing Evaluation for Correct Functioning of Arrhythmia Discrimination Algorithm in Dual Chamber ICD.

PACE 2000; 23: 496 (A) 
Dijkman B, Wellens H.J.J.

The VT: SVT Discrimination Algorithms in Dual Chamber ICDs - Improved But Still Not Perfect Automatic Arrhythmia Detection.

PACE 1999; 22: 614 (A)

Dijkman B, K. Den Dulk, Wellens H.J.J.

Clinical Experience with Paroxysmal Atrial Tachyarrhythmia in Patients Requiring an Automatic Mode Switching Pacemaker.

PACE 1995; 18: 354 (A).

Dijkman B, Den Dulk K, Wellens H.J.J.

Importance of Far Field QRS Sensing Evaluation for the Functioning of Automatic Mode Switching Algorithms.

PACE 1995:18: 136 (A).

Metzger J, Dijkman B, Vos M, Rodriquez, Smeets J, Gorgels A, Wellens H.J.J.

Triggered Activity as the Underlying Mechanism in Patients with Idiopathic Ventricular Tachycardia.

European Heart Journal 1993; 14:426 (A).

Gorgels A, Vos M, Smeets J, Dijkman B, Wellens H.J.J.

Use of Flunarizine to Indentify Triggered Activity as a Mechanism of Clinical Arrhythmias.

European Heart Journal 1991; 12:337 (A).

Smeets J, Rodriguez L, Cheriex E, Dijkman B, Wellens H.J.J.

Radiofrequente energie als behandeling voor hartritmestoornissen bij patieenten met extra atrioventricular verbinding.

Nederlandsch Tijdschrift voor Geneeskunde 1992;136:2131-35.

Gorgels A, Dijkman B, Smeets J, Vos M, Wellens H.J.J.

The Effect of Flunarizine on Supraventricular and Ventricular Tachycardias in Man. Journal of American College of Cardiology 1992; 19:63 (A).

Smeets J, Rodriquez L, Schlaepfer J, Katsivas A, Dijkman B, Xie Bayan, Meester A, Wellens H.J.J.

Can the ECG Differentiate between Ischemic and Non-Ischemic Ventricular Tachycardia?

Circulation 1991; 84: 2581 (A). 
Xie Bayan, Smeets J, Rodriquez L, Schlaepfer L, Dijkman B, Katsivas A, Penn O, Wellens H.J.J.

Clinical Atrial Fibrillation in Wolf-Parkinson-White Syndrome Patients Before and After Surgical Ablation.

European Heart Journal 1991; 12:278 (A). 